\title{
ELECTROMAGNETICALLY ASSISTED SHEET METAL STAMPING
}

\author{
DISSERTATION \\ Presented in Partial Fulfillment of the Requirements for \\ the Degree Doctor of Philosophy in the Graduate \\ School of The Ohio State University
}

By

Jianhui Shang, M.S.

$* * * * *$

The Ohio State University

2006

Dissertation Committee:

Approved by

Dr. Glenn S. Daehn, Adviser

Dr. Nitin Padture

Adviser

Dr. Katharine Flores

Graduate Program in Materials Science and Engineering 


\begin{abstract}
Sheet metal stamping is an important manufacturing process because of its high production rate and low cost. It is a fundamental technology in automotive, heavy vehicle and aerospace manufacture. A successful sheet metal stamping is to convert an initially flat metal sheet into a useful part with the desired shape. Stamping failures consist of either tearing (excessive tension) or wrinkling (excessive compression).

A new technology, hybrid electromagnetically assisted sheet metal stamping, was developed to control the strain distribution in a stamping operation to avoid failures. By embedding electromagnetic coils in conventional forming tools, controlling of the strain distribution of parts is enabled. Different applications of this new technology to sheet metal stamping are presented. And to better understand and utilize the new technology, analytical analyses for some application are also presented. This work focuses on the practical applications of this new technology.
\end{abstract}


Dedicated to my wife 


\section{ACKNOWLEDGMENTS}

The six-year study and research in the Ohio State University have been a great treasure for my life. I would never forget this valuable experience. I would like to take this chance to thank everyone who helped me in the past years.

First, I express my deep gratitude to my advisor, Professor Glenn Daehn, for his continuous support and guidance through the past six years. His endless creative ideas have been an important source of my research. I appreciate academic inspiration and personal help he provided to me. Without his encouragement and guidance, I would not finish this research.

I am grateful to my parents and my sisters for their constant support. They are always there for me without reservation. Their love and understanding helped me get through tough times. I owe great appreciation to my wife, Dong Wang. I am full of gratitude to her love, encouragement and patience. I know I can always count on them. I would be nowhere without their support. Love you all, my whole family.

I would like to thank Dr. Vincent Vohnout for his valuable suggestions and help. Also I am thankful to my research group members Manish Kamal, Mala Seth, Anthony Turner, Eduardo Del Rio, Scott Golowin, Kinga Unocic and Yuan Zhang, for their helps. 


\section{VITA}

February 19, 1972

1993

$1993-1995$

2000

2000- Present
Born- Hebei China

B.E., Metallurgy

Heibei Institute of Science and Technology, China Engineer

Xintai Iron and Steel Company, Xingtai, China

M.E., Materials Processing Engineering

University of Scince and Technology Beijing, China

Graduate Research Associate

The Ohio State University, Columbus, $\mathrm{OH}$

\section{PUBLICATIONS}

1. V. Vohnout, Jianhui Shang and G.S. Daehn, "Improved Formability by Control of Strain Distribution in Sheet Stamping using Electromagnetic Impulses", Proceedings of the $1^{\text {st }}$ International Conference on High Speed Forming, March 31April 1, Dortmund, Germany, pp. 211-221, (2004).

2. G. S. Daehn, Jianhui Shang and V. Vohnout, "Electromagnetically Assisted Sheet Forming: Enabling Difficult Shapes and Materials by Controlled Energy Distribution”, Proceedings from Energy Efficient Manufacturing Processes, March 2-6, San Diego, C.A. pp. 117-128, (2003)

3. Shang Jianhui, Wang Xianjin, Jiang Dongmei, Kong Bingyu and Lu Jiangxin, "Effect of Coiling Temperature on Precipitation Behavior of Second-phase Particles and Grain Sizes in Ti-IF Steel”, Iron and Steel (China), Vol. 37, No. 3, pp. 43-47. March, (2002) 
4. Shang Jianhui, Wang Xianjin, Ru Zheng and Zhong Dingzhong, "Effect of Total Finish-rolling Strain and Pass Strains on Precipitating Behavior in Ti-IF Steels", Journal of University of Science and Technology Beijing, Vol. 22, No.1, pp. 44-48, (2000)

\section{FIELDS OF STUDY}

Major Field: Materials Science and Engineering 
TABLE OF CONTENTS

Abstract ....

Chapters:

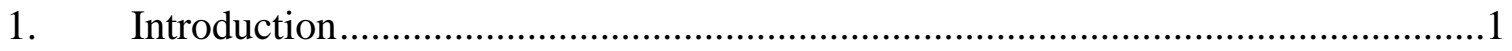

2. An overview of sheet metal stamping and electromagnetic forming ....................5

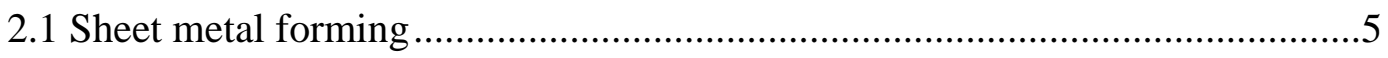

2.2 Main methods to improve formability of stamping ....................................10

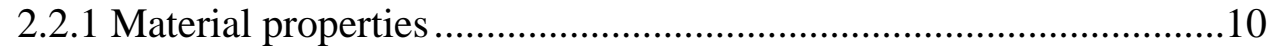

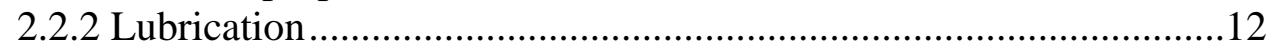

2.2.3 Varied blank holder force ............................................................15

2.2.4 Multi-point die-cushion technology............................................16

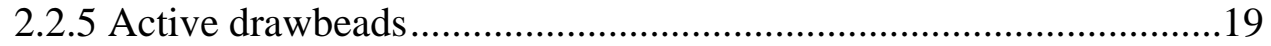

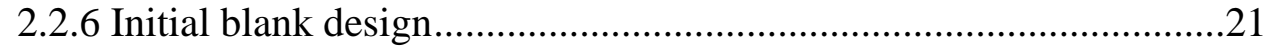

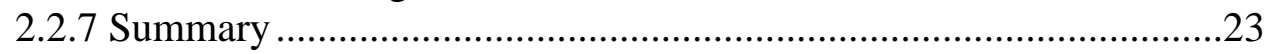

2.3 Electromagnetic forming ......................................................................24

2.3.1 Fundamental of electromagnetic forming ...................................24

2.3.2 Some important features of electromagnetic forming ....................27

2.3.3 Benefits of electromagnetic forming ..........................................28

2.3.4 Applications of electromagnetic forming .....................................30

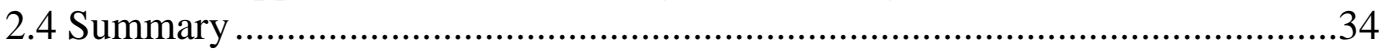

3. Elements of electromagnetically assisted sheet metal stamping..........................36

3.1 Electromagnetically assisted sheet metal stamping ...................................36

3.1.1 Strain states of sheet metal stamping ..........................................36

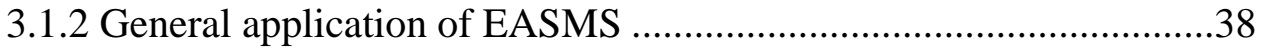

3.2 Engineering design and optimization for EASMS........................................41 
4. Application of electromagnetic forming at flange in deep drawing ....................45

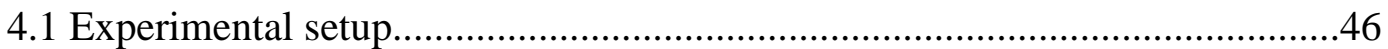

4.1.1 Initial analysis of deep drawing .................................................46

4.1.2 Deep drawing tool.................................................................48

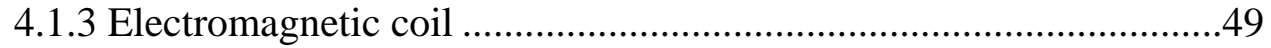

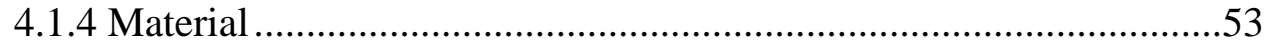

4.1.5 Electromagnetic energy determination ......................................54

4.1.6 Experiment procedures ..............................................................58

4.2 Experiment results and discussions .......................................................59

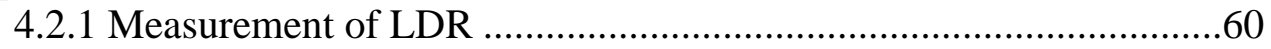

4.2.2 Blanks with 101.6mm diameter ................................................61

4.2.3 Blanks with 127.0mm diameter ...................................................63

4.2.4 Blanks with $177.8 \mathrm{~mm}$ diameter ..................................................74

4.2.5 Blanks with $254 \mathrm{~mm}$ square..................................................76

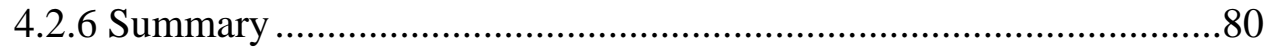

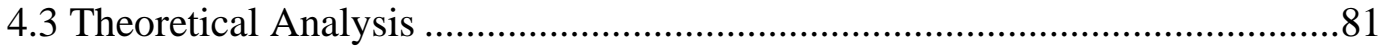

4.3.1 Theoretical analysis of conventional deep drawing......................83

4.3.2 Theoretical analysis of electromagnetically assisted deep drawing .91

4.3.3 Electromagnetic pulse design ................................................111

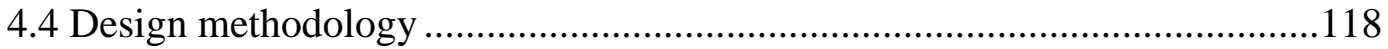

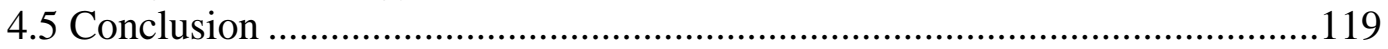

5. Electromagnetic forming to increase drawing in for lip-skin panel ..................120

5.1 Analysis of lip-skin panel production ......................................................120

5.2 Design of electromagnetically assisted sheet metal forming.........................123

5.2.1 Approach design ....................................................................123

5.2.2 Tooling design ...............................................................124

5.2.3 Electromagnetic coil design .....................................................126

5.2.4 Blank material and size design ................................................128

5.2.5 Experiment procedure ........................................................130

5.3 Experiment results ..........................................................................132

5.3.1 Stamping with conventional stamping and EM forming ...............132

5.3.2 Stamping with shimming at inner side ......................................138

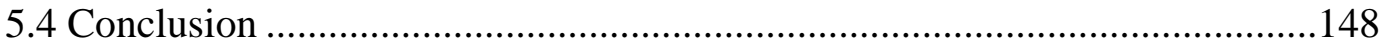

6. Application of electromagnetic forming at bottom of metal part ......................150

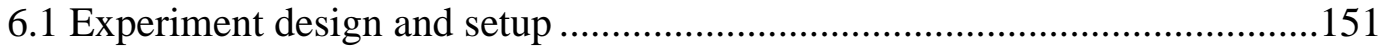

6.1.1 Press and tooling ................................................................151

6.1.2 Electromagnetic coil and punch...............................................153

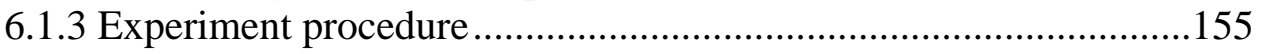

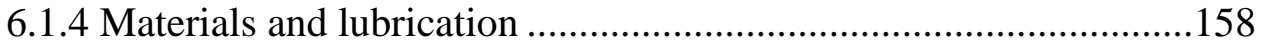

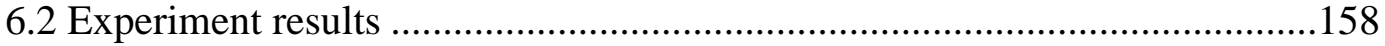


6.2.1 A typical result ............................................................................158

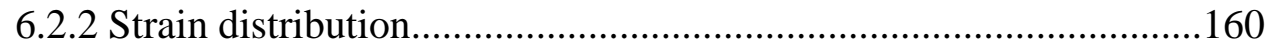

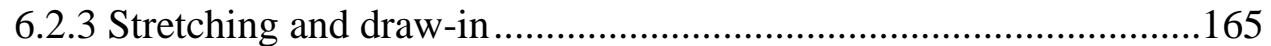

6.2.4 Stretching only ...............................................................................168

6.2.5 Effect of coil configuration ...........................................................172

6.2.6 Effect of process parameters ........................................................174

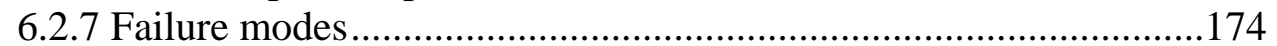

6.2.8 Bottom region waviness...............................................................176

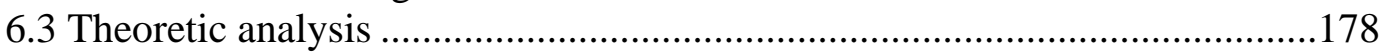

6.3.1 Straight side analysis.....................................................................181

6.3.2 Corner side analysis ...................................................................186

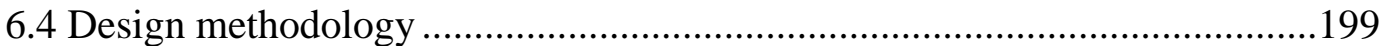

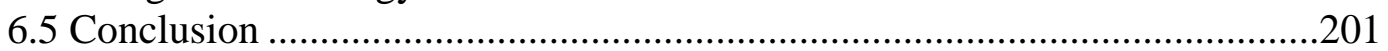

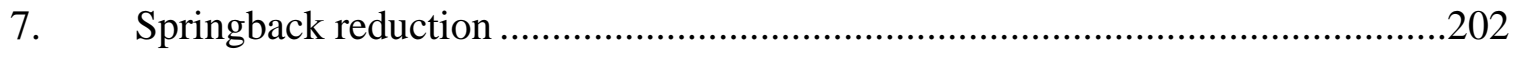

7.1 Introduction to springback ......................................................................202

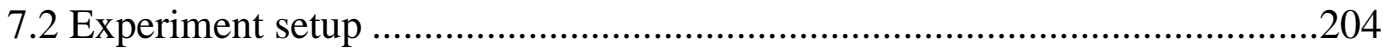

7.3 Experiment results .................................................................................207

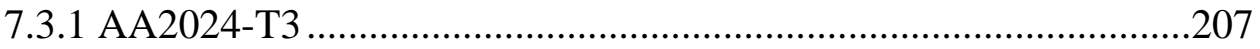

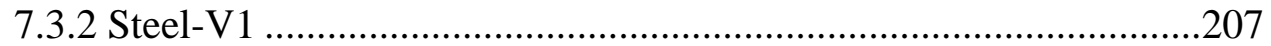

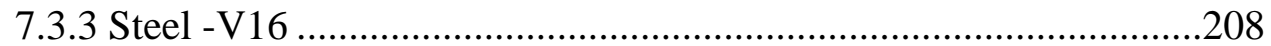

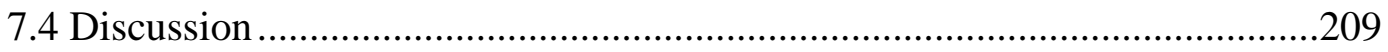

7.4.1 Materials properties .....................................................................209

7.4.2 EM coil geometry ……………………………….....................212

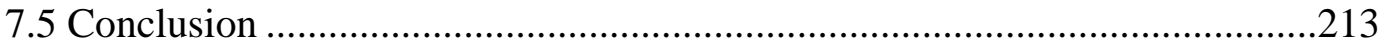

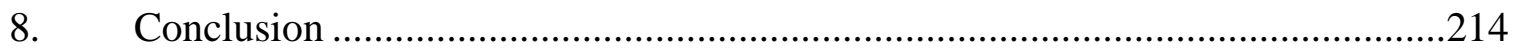

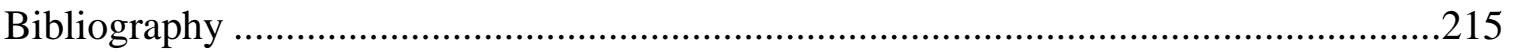




\section{LIST OF TABLES}

Table $\quad$ Page

4.1 Deep drawing tool geometric parameters ...................................................49

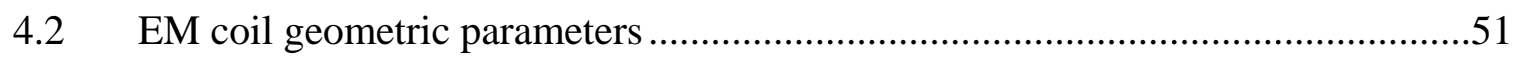

4.3 Mechanical tensile properties of AA2219-O sheet material................................54

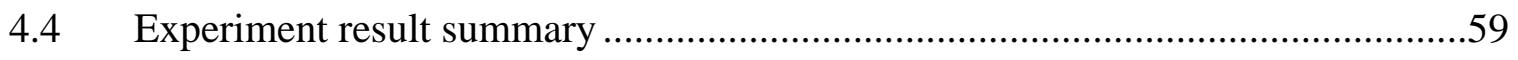

4.5 Flange sizes and cup height of samples with different EM energy level...............73

4.6 The calculation results from the predict model.............................................117

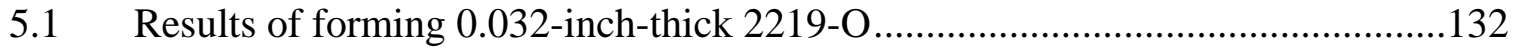

5.2 Forming 0.032” 2219-O with shimming at inner side flange ............................142

6.1 Summary of improvement of draw in and side wall length at different sides .....166

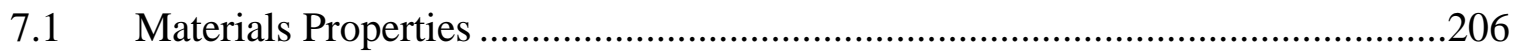




\section{LIST OF FIGURES}

Figure Page

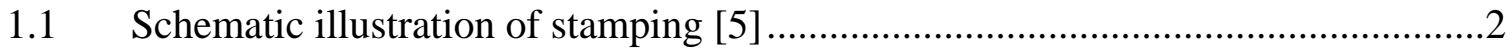

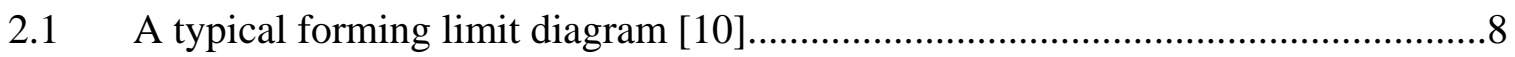

2.2 Forming limit diagram with wrinkling limit curve [18] .....................................

2.3 Distribution of strains in the sheet stretching for several materials (The punch was stopped at the same peak strain, but the cup depth increases as the strain is more widely distributed. Brass has the highest $n$ and aluminum the lowest.) [7] .........12

2.4 Friction effects on radial strain profiles along radial direction in simulating stretch forming behavior (punch travel distance $25 \mathrm{~mm}$ ) [13] ......................................14

2.5 Schematic of a stamping process [18] $\mathrm{F}_{\mathrm{p}}$ : Punch force; $\mathrm{F}_{\mathrm{b}}$ : Blank holder force; $\mathrm{F}_{\mathrm{r}}$ : Restraining force acting on the drawn blank .....................................................15

2.6 Multi-point-control unit for production of bowl kitchen sinks [37] .....................18

2.7 Drawing characteristics of a bowl stainless kitchen sink [37] ...........................18

2.8 (a) Limit curves obtained for different drawbead trajectories; (b) Comparison of strain distributions between different drawbead trajectories [38] .........................20

2.9 Experiment results for the optimal blank (trapezoidal cup) [46] .........................23

2.10 Schematic diagram of electromagnetic forming .............................................24

2.11 The typical traces of primary and induced currents........................................25

2.12 Typical images of electromagnetic forming process (Vave. $=200 \mathrm{~m} / \mathrm{sec}, 50 \mathrm{E}-6$ sec between images) [6] ......................................................................27

2.13 Forming limit diagram with high velocity forming data [53]...........................29 
2.14 Electromagnetic forming application [78]: (a) tube compression; (b) tube expansion; (c) sheet or pan forming.

2.15 Matched tool-electromagnetic hybrid sheet forming [6] .......................................32

2.16 Automotive inner-door panels, forming demonstration [6] ..................................33

2.17 The electromagnetic re-forming process carried out on the door panel [79]..........33

3.1 Schematic of strain states in a stamped metal part [5] ...........................................37

3.2 Schematic of electromagnetically assisted sheet metal forming [1] ......................38

3.3 Design and optimization approach for electromagnetically assisted sheet metal stamping ........................................................................................................4

4.1 Contour of the maximum principal strain in plane for a sheet metal stamping with large initial blank size ............................................................................................4

4.2 Conventional axisymmetric deep drawing.........................................................48

4.3 Electromagnetically assisted deep drawing ……...................................................49

4.4 Electromagnetic coil embedded in the die of deep drawing ..................................51

4.5 Photograph of the electromagnetically assisted deep drawing setup (the tooling and the coaxial coil) …………………………………........................................52

4.6 Photograph of the 48KJ capacitor bank ............................................................52

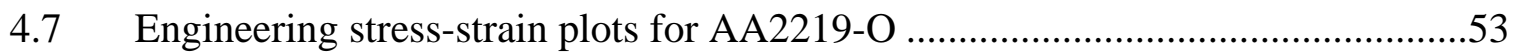

4.8 Photo of the initially flat metal sheet with $127 \mathrm{~mm}$ diameter after one $4.2 \mathrm{~kJ}$ EM

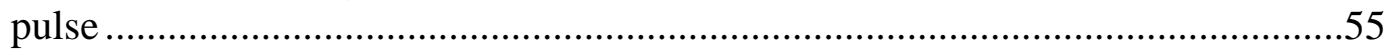

4.9 Primary current trace in the EM coil for $4.2 \mathrm{~kJ}$ EM pulse.......................................56

4.10 Contour of the current density distributions of primary and induced currents ......57

4.11 Contour of EM force density distribution in metal sheet.........................................58

4.12 Photograph cup drawn from 95.3mm-diameter blank (2224N clamp load, 30.2mm cup height, and with lubrication; Material: AA2219-O) ..........................................61 
4.13 Photograph of cups drawn from 101.6mm diameter blank (Left: cup height $10.4 \mathrm{~mm}$ with 2224N clamp load made by conventional deep drawing; Right: cup height $34.5 \mathrm{~mm}$ with $2224 \mathrm{~N}$ clamp load and 27 pulses)

4.14 Photograph of cups drawn from 127.0mm diameter blank (Left: 8.6mm cup height with $1334 \mathrm{~N}$ clamp load made by conventional deep drawing; Right: $30.9 \mathrm{~mm}$ cup height with $1334 \mathrm{~N}$ clamp load and 25 electromagnetic pulses) .......64

4.15 Comparison of the flange size after deep drawing, same samples in Figure 4.13 (Left: 126mm diameter; Right: 113mm diameter; Original: 127mm diameter)....65

4.16 Large stretching on the flange caused by EM pulses (same sample shown in right of Figure 4.15) .67

4.17 Comparison of strain distributions..................................................................68

4.18 Comparison of strain distributions and the punch load ..........................................69

4.19 Photograph of cup drawn without wrinkling at flange (with original $127.0 \mathrm{~mm}$ diameter blank, 2669N clamp load and 24 EM pulses; after tearing at flange, cup height is $29.9 \mathrm{~mm}$ and flange diameter is $117 \mathrm{~mm}$ ).............................................70

4.20 Photograph of cups drawn from $127.0 \mathrm{~mm}$ diameter blank with different EM energy levels and same clamp load (Left: $11.4 \mathrm{~mm}$ cup height with 10 times of 3.0KJ EM pulses; Right: 24.8mm cup height with 20 times of 6.0 KJ EM pulses; Middle: $30.9 \mathrm{~mm}$ cup height with 25 times of $4.2 \mathrm{KJ}$ EM pulses). .73

4.21 Photographs of cups drawn from blanks with $177.8 \mathrm{~mm}$ diameter (Left: $7.7 \mathrm{~mm}$ cup height with 2669N clamp load made by conventional deep drawing; Right: $19.1 \mathrm{~mm}$ cup height with $2669 \mathrm{~N}$ clamp load made by 18 times of EM pulses).....75

4.22 Photograph of cups drawn from $254 \mathrm{~mm} \times 254 \mathrm{~mm}$ square blanks (Left: $7.7 \mathrm{~mm}$ cup height with $4000 \mathrm{~N}$ clamp load made by conventional deep drawing; Right: $17.8 \mathrm{~mm}$ cup height with $4000 \mathrm{~N}$ clamp load made by 16 times of EM pulses).....76

4.23 Photograph of the flanges of cups (Left: made by conventional deep drawing same as the left in Figure 4.22; Right: made by electromagnetically assisted deep drawing same as the right in Figure 4.22)............................................................78

4.24 Photograph of the cups drawn $254 \mathrm{~mm} \times 254 \mathrm{~mm}$ square blanks (Left: $5.4 \mathrm{~mm}$ cup height with $1334 \mathrm{~N}$ clamp load made by conventional deep drawing; Right: $17.8 \mathrm{~mm}$ cup height with $1334 \mathrm{~N}$ clamp load made by 16 times of EM pulses).....79

4.25 Schematic representation of a partial drawn cup [91] .85 
4.26 Radial stresses in the flange element [91].

4.27 Schematic part drawn cup of electromagnetically assisted deep drawing ( $r_{0}$ is the current radius of blank, $r_{c}$ is the radius of coil center, $r_{e}$ is the edge radius of the hollow groove in blank holder, $w$ is the width of coil and $\Delta r$ is the draw-in caused by electromagnetic pulse)

4.28 Partial schematic of stretching and draw-in caused by electromagnetic pulse ......95

4.29 Partial drawn cup of electromagnetically assisted deep drawing ......................100

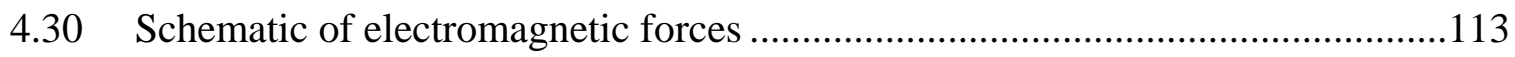

5.1 Photograph of a completely formed and untrimmed lip skin (The inner and outer arc lengths are noted).

5.2 Photographs of the grids and formed part with the 5 sub-sections that were cut out labeled.....

5.3 Major strains as a function of position for the fully formed lip-skin panel .........122

5.4 Assembly drafts of steel tooling and the press.............................................124

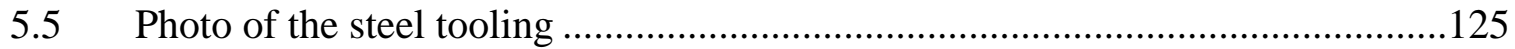

5.6 Photo of punch and clamp holder in hydraulic press .....................................125

5.7 Electromagnetic coil (Made of Cooper 110) insulated by Kapton tape...............127

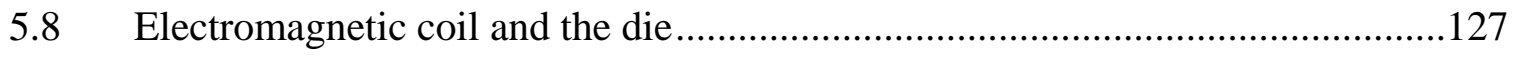

5.9 G10 plate, punch and upper clamp holder in hydraulic press ...........................128

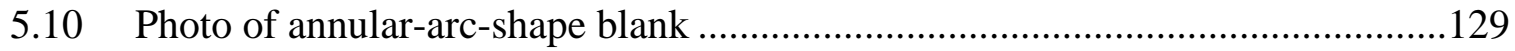

5.11 Photo of part after stamping with annular-arc-shape blank .............................129

5.12 Polygon blank with 18-inch length and 16-inch width...................................130

5.13 Photo of sample 1\# (Draw depth: 2.580 inches, serious wrinkling on inner)......132

5.14 Photo of sample 3\# (draw depth: 1.563 inches, tearing on outer side)...............133

5.15 Photo of sample 2\# (Clamp load: 10 klbs; Draw depth: 2.189”; 21 EM pulses with $2.4 \mathrm{~kJ}$ per pulse) 
5.16 Inner side of Sample 4\# (Clamp load: 30 klbs; Draw depth: 2.482”; 17 pulses with $1.2 \mathrm{~kJ}$ per pulse)

5.17 Outer side of Sample 4\# (Clamp load: 30 klbs; Draw depth: 2.482”, 17 EM pulses with $1.2 \mathrm{~kJ}$ per pulse) .........................................................................................136

5.18 Comparisons of Sample 3\# and 4\# ................................................................136

5.19 Punch load and punch stroke of Sample 1\# 4\#............................................137

5.20 Paper for shimming at the inner side clamp.......................................................139

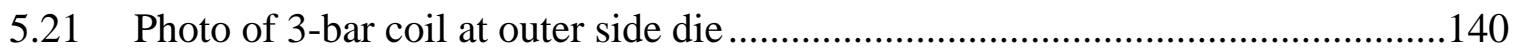

5.22 New 0.032-inch-thick 2219-O (Clamp load: 30 klbs; Draw depth: 2.633”; wrinkling appeared at the inner side wall) .141

5.23 Sample 6\# (Clamp load: 30 klbs; Draw depth: 1.930”; Tearing at the inner side wall; with shimming at the inner side flange)

5.24 Photo of sample 9\# (Clamp load: 30 klbs; Draw depth: 3.376 inches; 120 EM pulses with $1.2 \mathrm{~kJ}$ per pulse; Wrinkling at the inner side wall; shimming at the inner side)

5.25 Photo of sample 10\# (Clamp load: 30 klbs; Draw depth: 3.376 inches; 50 EM shots with $2.4 \mathrm{~kJ}$ per shot; Wrinkling at the inner side wall; shimming at the inner side). .146

5.26 Photo of sample 13\# (Clamp load: 30 klbs; Draw depth: 2.042"; 21 pulses with $2.4 \mathrm{~kJ}$ per pulse; inner side coil; shimming at inner side flange)

6.1 Photo of hydraulic press and tooling used in experiments ...................................152

6.2 Dimensions of fully formed scale-model fender panel [81] ................................152

6.3 Photo of the punch and the embedded electromagnetic coil..................................153

6.4 Connection between capacitor bank and electromagnetic coil inside of punch ..154

6.5 Experiment set-up (capacitor bank and press) .....................................................155

6.6 Schematic procedure of (a) electromagnetically assisted stamping; (b) conventional stamping 156 
6.7 Photos of experiment results (Left: typical result made by conventional stamping; Right: typical result made by electromagnetically assisted stamping) .................158

6.8 Punch load curves for the two samples shown in Figure 6.7 ………………........159

6.9 Locations of $\mathrm{x}$ and $\mathrm{y}$ lines on metal part ..........................................................160

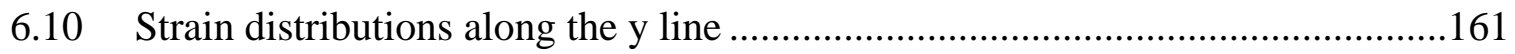

6.11 Strain distributions along the $\mathrm{x}$ line ...............................................................162

6.12 Photos of the corner regions and the locations of measure lines (Left: electromagnetic assisted stamping; Right: conventional stamping) .....................163

6.13 Comparison of strain distributions of corner region .............................................164

6.14 Measured strain states for both cases, compared to FLD for 6111-T4 [6] ...........165

6.15 Photos of the straight side wall for conventional and EM assisted stamping ......166

6.16 Photos of the corner side wall for conventional and EM assisted stamping........166

6.17 Comparison of improvement of draw-in and contribution of draw-in .................167

6.18 Different locations shown in Figure 6.17 .....................................................168

6.19 Photos of the samples with $1245 \mathrm{kN}$ clamp load (Left: conventional stamping, draw depth is 25.3mm; Right: EM assisted stamping, draw depth is $31.0 \mathrm{~mm}$ ) ..169

6.20 Photos of the straight side walls for conventional (left) and EM assisted stamping

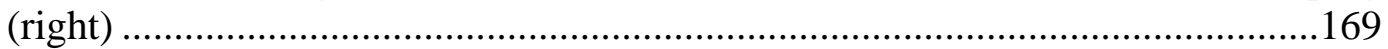

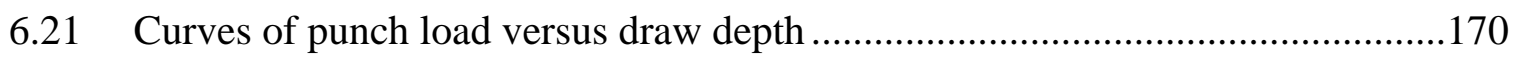

6.22 Comparison of effect of clamp load on draw in and draw depth..........................171

6.23 Photos of coil configurations (Left: configuration 1; Right: configuration 2).....172

6.24 Strain distributions along $\mathrm{x}$ line with coil configuration 2 .................................173

6.25 Tearing at the bottom region............................................................................

6.26 Measured strains near the crack, compared to FLD for Al 6111-T4 [6] .............176

6.27 Scanned surface shape of bottom region along $\mathrm{x}$ line............................................177 
6.28 Plan view of a quarter of a box [112]

6.29 Half-section of straight side of a partially drawn box by conventional stamping $[88,112]$

6.30 Schematic half-section of straight side of a partially drawn box by EM assisted stamping.

6.31 Schematic corner side of a partially drawn box by conventional stamping ........187

6.32 Schematic corner side of a partially drawn box by EM assisted stamping..........189

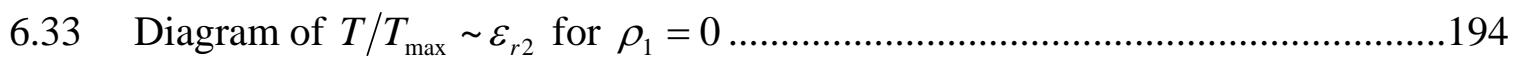

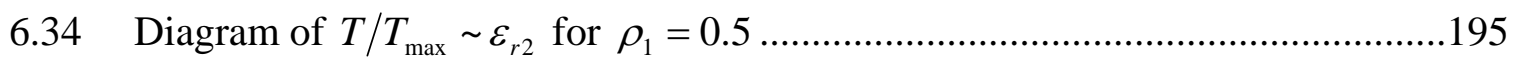

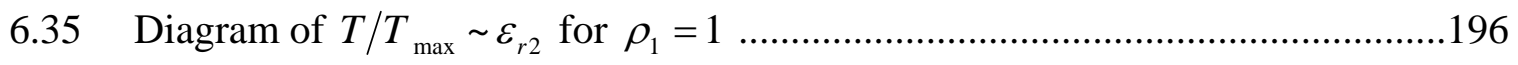

6.36 A typical current trace for 5.4kJ in EM coil ................................................197

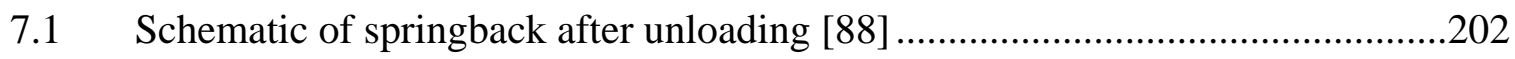

7.2 Photo of single-turn coil used in experiments................................................205

7.3 Photo of steel female die on the top of single turn coil ...................................205

7.4 Bent metal blank on the top of single turn coil (After bending and electromagnetic forming, the steel die was removed) ..............................................................206

7.5 Photo of bent AA2024-T3 blanks and the relationship between EM energy and springback angle 207

7.6 Photo of the bent steel-V1 blanks and the relationship between EM energy and springback angle

7.7 Photo of the bent steel-V16 blanks and the relationship between EM energy and springback angle 209

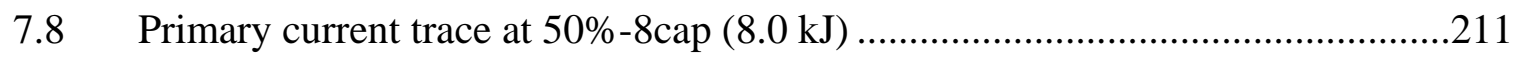

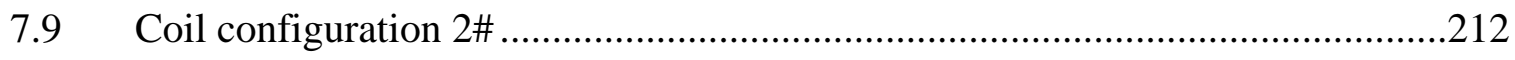

7.10 Schematic illustration about coil dimensions (a) Coil configuration 1\#; (b) Coil configuration 2\# (unit: inch; Red: EM coil; Blue: Urethane) .213 


\section{CHAPTER 1}

\section{INTRODUCTION}

Sheet metal stamping is an important manufacturing process because of its high production rate and low cost. It is a fundamental technology in automotive, heavy vehicle and aerospace industries. It is also important in appliance manufacture and has an increasing role in making cases and sub-structures for electronics such as computer cases, cell phones and avionics. In the case of vehicles, fabricated sheet metal components have a strong role in determining the ultimate strength, weight and crashworthiness of the assembled vehicle [1].

Current initiatives in automotive industry are driving a need for stronger and lighter automotive panels [2]. Light metals, such as high strength steel, aluminum alloys, magnesium, are developed to reduce overall body weight and improve the fuel consumption [3]. However, their lower formability tends to create considerable problems. Simultaneously, car designers are eager to manufacture the body panels with increasingly complex shapes. Together, these two factors have pushed the current sheet metal stamping technologies to their limits [2]. 
A successful sheet metal stamping process converts an initially flat metal sheet into a useful part with the desired shape. The formability of a sheet metal is a complex measure of its ability to accommodate the strains for the desired shape without failure in a forming process. Formability depends on not only the properties of the sheet metal, but also the process factors in a practical forming operation [4]. Figure 1.1 shows a schematic illustration of the stamping process [5]. The basic components of stamping are a punch, a die (some kinds of stamping such as stretch forming may not include it) and a set of blank holders that may or may not include drawbeads [5]. The punch draws the initially flat blank into the die cavity to form the shape, and the blank holder restrains the flange of the blank to prevent wrinkling and to control the metal flow into the die cavity.

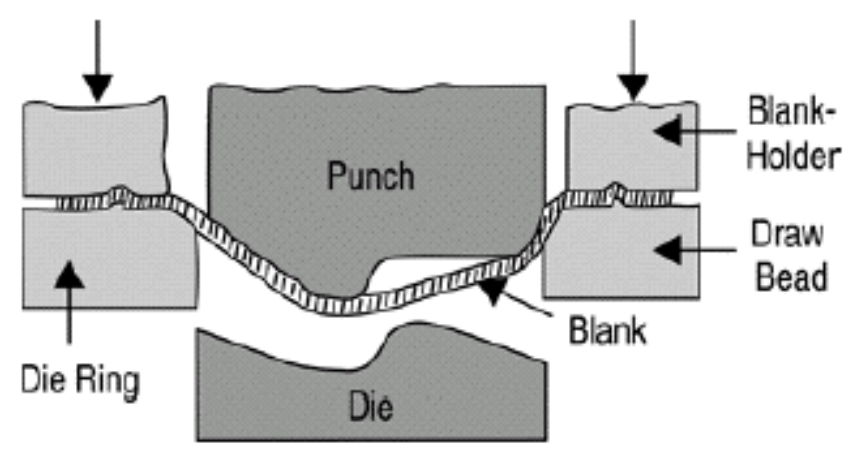

Figure 1.1 Schematic illustration of stamping [5]

Failure in the stamped parts is caused mainly by either wrinkling or tearing. Wrinkling, which often occurs in the flange inside the blank-holder or in the unsupported sidewall of 
the stamped part, is due to excessive compressive stresses in the plane of the sheet that result in bulking of the sheet [2]. Tearing, on the other hand, is nominally caused by excessive tensile stresses in the plane of the sheet. So to accomplish the desired deformation without failure, there are generally two ways to apply. One is to use materials with better properties to resist wrinkling and tearing, which needs to develop new materials with high mechanical properties. Another one is to control the strain distribution of the stamped part. The ideal strain distribution is uniform without excessive compressive strains and excessive tensile strains. Therefore, the metal sheet can provide sufficient strains for the desired shape and does not reach its local failure criteria.

Many manufacturing methods have been developed to improve the formability in sheet metal stamping, such as active drawbead control, active blankholder force control, lubrication and blank optimization. They may be applied together or individually. Although all of above methods have utility, they are basically applied to control the metal flow and then to indirectly affect the strain distribution.

This dissertation expands on a completely new approach, electromagnetically assisted sheet metal stamping, to improve the formability of sheet metal, which is based on the idea of directly delivering the deformation where it is required and then controlling the strain distribution. The key idea of this approach is the incorporation of electromagnetic coils with conventional stamping tools, which enables the directly controlling of strain distribution of stamped parts. 
This dissertation begins with the review of methods to improve the formability of sheet metal stamping process and basic elements of electromagnetic forming in chapter 2 . Chapter 3 will introduce the basic elements and design approach of electromagnetically assisted sheet metal stamping. Application of electromagnetic forming at flange area of axisymmetric deep drawing and a 2D analysis of the forming process are detailed in chapter 4 . Chapter 5 deals with the application of electromagnetic forming at flange area during the lip-skin panel production. The application of this new approach at the bottom area of non-symmetric part is present in chapter 6. A simple analysis is also presented to understand this forming process. Chapter 7 demonstrates the feasibility of reducing springback of bending by electromagnetic forming. At last, chapter 8 summarizes the current works on the electromagnetically assisted sheet metal stamping. 


\section{CHAPTER 2}

\section{AN OVERVIEW OF SHEET METAL STAMPING AND ELECTROMAGNETIC FORMING}

\subsection{Sheet metal stamping}

Sheet metal stamping is the most common method of producing metal parts in the automotive industries, because of its high production rate and low cost. In basic sheet metal stamping, a flat metal sheet is pressed to the desired shape bewteen a die and a punch. The die is used to define the outside shape of the part. And the punch is applied to define the inside shape of the part [6]. Besides, there are usually the third component of tools called the blank holder, which is used to hold the blank against the die top and to control the blank draw-in. The sheet metal stamping is essentially a position control process [6]. During the stamping process, the punch moves to the predetermined position and stops. Then the part is fully shaped. In many practical operations, the blank holder should clamp the metal sheet flange before the punch moves. Since the punch movement can be controlled according its position, the press and the controls that are required for this forming operation can be quite simple. The most commonly used press machines are mechanical, which are relatively simple and inexpensive. There are also hydraulic presses, which are relative expensive. But they can provide independent control of speed and position of the tooling during the forming process [6]. 
Hydroforming, high velocity forming and superplastic forming methods are also used to manufacture sheet metal parts. But these methods need higher cost of production and longer cycle times than conventional stamping. They have low production rates for high volume manufacturing [1]. So they are not widely used in the manufacturing industries, comparing to sheet metal stamping. Therefore, this dissertation only focuses on the sheet metal stamping.

Sheet metal stamping has its forming characters. In sheet metal stamping, the predominant deformation is tensile in plane. And the through-thickness deformation is often compressive. During sheet metal stamping, one or both surfaces of some deforming regions are often free, i.e. not supported by tools [7]. Meanwhile, the sheet metal is so thin that it is easy to wrinkle. These two factors make sheet metal stamping have strong wrinkling tendency. During sheet metal stamping, the part flange is usually held firmly enough with the blank holder so that the part flange can resist wrinkling. Then the punch pushes the sheet into the die cavity, draws materials into the cavity and stretches it.

In sheet metal stamping, different deformation modes can operate in different areas of metal sheet [8]. There are two basic deformation modes, drawing and stretching. For drawing, one of the principal strains in the plane of the sheet is positive and the other is negative, and the change of thickness is small. For stretching, two of the principal strains are tensile, and the thinning is required. In stamping, stretching may predominate in one region while drawing prevails in another [7]. The relative amounts of drawing and stretching vary in different stamping processes and different stamping stages. 
Forming limit diagram (FLD) is a common laboratory and industrial method to use in sheet forming formability analysis [9]. In this technique, circle grids are marked on the original sheet surface, which will change to ellipse after the deformation. The major and minor surface strains can be measured by comparing the major and minor axes of the grid before and after the forming operation. The forming limit of sheet metal is defined to be the state at which a localized thinning of the sheet initiates during forming, ultimately leading to a split in the sheet [9]. To analyze an actual sheet forming operation, local strains near failures or suspected trouble points are measured and compared with the FLD. If the strains are near the failure curve, failures are likely to occur during the forming process. Figure 2.1 displays a typical forming limit diagram [10]. From this diagram, two methods to avoid these trouble points can be seen. One is to change the FLD curve and provide higher limit strains, which means a better material. Investigations $[7-12,17]$ on the FLD curves show that both strain rate hardening and strain hardening have strong effect on the limit strains.

The other one is to change strain states. The lowest major strain value on a typical FLD always occurs at or near plane strain state, in which minor strain is zero [13-17]. If the deformation mode in a critical region is at or near plane strain state, it will be helpful to design the forming process so that the critical region undergoes either more compressive stress in plane or more biaxial stretching to avoid plane strain state [9]. Therefore, controlling the strain distribution would be very useful to avoid failure during the forming. 


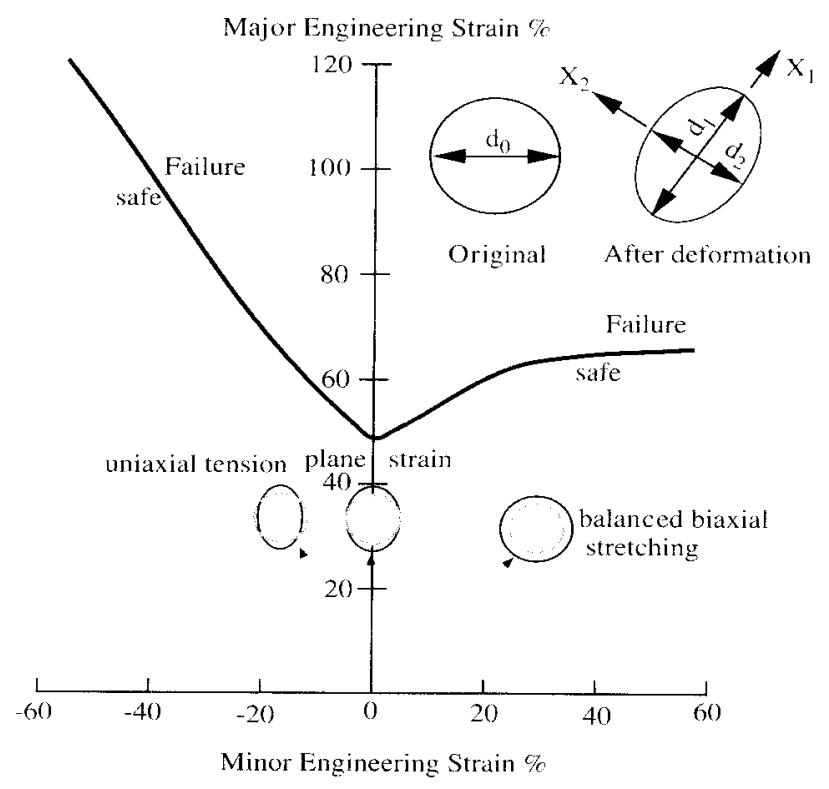

Figure 2.1 A typical forming limit diagram [10]

There are two basic failure in sheet metal stamping, tearing and wrinkling. Forming limit diagram (FLD) denotes the tension strain limits for tearing. Efforts were made to find a similar strain limits to express the onset of wrinkling due to compressive instability [17]. Figure 2.2 shows a forming limit diagram with wrinkling limit curve (WLC) [18]. This diagram also shows that there are two ways to avoid wrinkling. One is to change the wrinkling limits. The other one is to change the strain states to avoid excessive compression. 


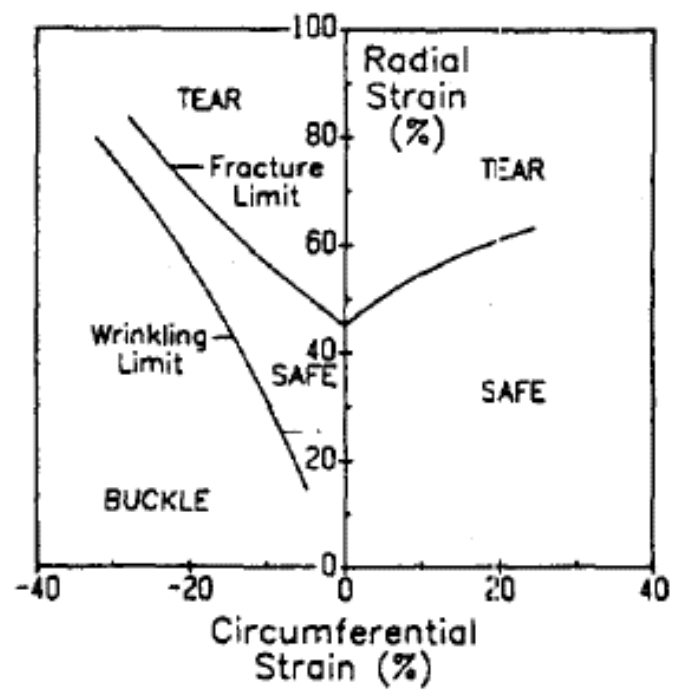

Figure 2.2 Forming limit diagram with wrinkling limit curve [18]

\begin{abstract}
Above analysis indicates that controlling the strain distribution to avoid the strain states near forming limits is the only way for a given material and a given shape of parts in sheet metal stamping. If the strain states above forming limits can not be avoided, this stamping will be failed. Many methods have been developed to improve the formability of stamping. The majority of them focus on devising ways to control the metal flow and then to affect the strain distribution [1]. A brief review of these methods will be presented next.
\end{abstract}




\subsection{Main methods to improve formability of stamping}

\subsubsection{Material properties}

The better material properties of sheet metals can surely improve the formability of stamping. They can improve the ability to resist wrinkling and tearing. Some important mechanical properties will be introduced below.

Strain hardening is the ability of materials to strengthen or harden with increasing plastic strain level. It is one of the most important properties influencing the formability of sheet metals. During plastic deformation, a region undergoing thinning can resist further deformation by virtue of strain hardening and can spread deformation to its neighboring regions, thus promoting uniform thinning [19]. One parameter reflecting this behavior is strain hardening exponent, $n$, defined as $n=\partial \ln \sigma / \partial \ln \varepsilon$, for the materials obeying the power law [20]. For the sheet metal stamping, the value of strain hardening exponent $n$ almost equals the critical strain prior to the onset of diffuse necking [7-9, 35-36]. A good formability of a sheet metal requires a high $n$ value and also the maintenance of the high $n$ value up to large enough strains during deformation [19].

Strain rate hardening is the resistance of materials to strain localization through the accommodation of strain rate change. As strain localization initiates, the local strain rate accelerates to maintain compatibility with the far-field displacement [19]. The parameter

reflecting this behavior is strain rate sensitivity, $m$, defined as $m=\partial \ln \sigma /\left.\partial \ln \dot{\varepsilon}\right|_{\varepsilon}$ [7-9]. A positive value of $m$ lowers the growth rate of strain and strain rate gradients after the onset of instability like diffuse necking, whereas a negative value of $m$ would accelerate 
the strain localization[19]. So higher strain rate sensitivity $m$ could increase the postuniform strains, and then increase the formability of metals.

Anisotropy of a sheet metal is the third factor that may impose effects on formability. The very useful parameter for sheet metal is the plastic anisotropy $R$, defined as $R=\varepsilon_{w} / \varepsilon_{t}$ (the ratio of plastic strain of the width direction to that of the thickness direction in the tensile specimen) [7-9, 36]. A high $R$ value indicates that the material has a high thinning resistance. The thickness of sheet metal usually is small, so a higher $R$ value is better for sheet metal stamping.

Moreover, materials properties also influence the strain distribution of stamping parts. During the sheet metal stamping, the deformation is inhomogeneous because of the tooling geometry, the friction and so on. The strain states and stress states are different at different areas. There always exist strain and stress gradients in the metal sheet. So with enhanced values of $m$ and $n$, the materials have more resistances to the gradients and can decrease the peak values of strain. Then the strain distribution can be more uniform. The results in Figure 2.3 show that increasing $n$ tends to distribute the strain more widely and permit deeper parts to be formed [7]. 


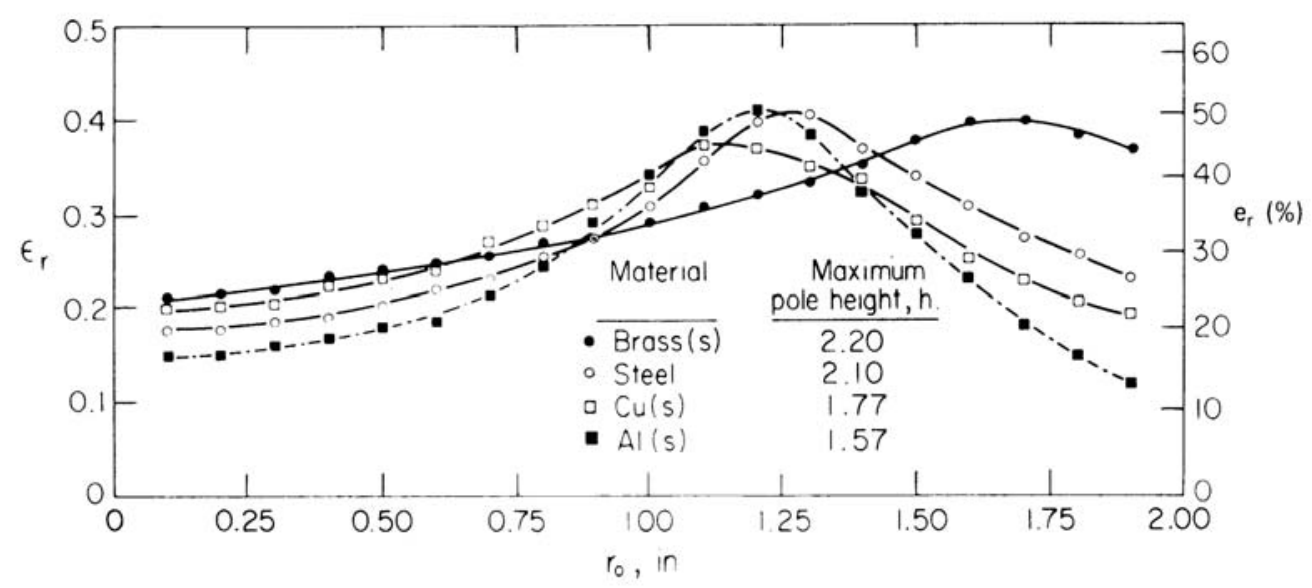

Figure 2.3 Distribution of strains in the sheet stretching for several materials (The punch was stopped at the same peak strain, but the cup depth increases as the strain is more widely distributed. Brass has the highest $n$ and aluminum the lowest.) [7]

However, requiring better materials reduces the material selection and increases the production cost. More often, the problem met in sheet metal stamping is to produce parts from given materials.

\subsubsection{Lubrication}

Lubrication between the blank and tools plays an important role in controlling the metal flows and affecting strain distributions for sheet metal forming. Lubrications can reduce friction between tools and the metal sheet, and benefit the metal flows. Proper lubrication can provide suitable metal flow into die cavity. As the friction decreases, the stress distribution in the metal sheet will become more uniform, eliminating localization. Correspondingly, the strain distribution will become more uniform and the formability 
can be improved. In addition, reduced friction force means smaller drawing forces and usually better part surface finish [7].

There are some studies addressing the effects of lubrications on the formability and strain distribution of sheet metal [21-24]. J. Wei et al [22] compared effects of lubricants on the aluminum sheet metal forming. They tried different lubricants that have different friction coefficients in a deep drawing test. Their experiment results demonstrated that the type of lubricants has greatly effect on the strain distribution. They found that the boric acid dry films could bring greater strain distribution than commercial liquid and solid lubricants, because of their lower friction coefficient $(\approx 0.04)$ and high adhesion strengths to aluminum alloys. Xia and Llewellyn [13] studied the effect of the coefficient of friction on strain distribution of punch stretching by FEM and experiment. The predicted results of FEM in Figure 2.4 were generally in agreement with the experiment, and showed that strain distributions are very sensitive to changes in coefficient of friction. The lower coefficient of friction is, the higher strain level at the punch head is, and the lower the peak strain value is. Under the same limits of failure strain, the lower coefficient of friction leads to greater strain distribution, which indicates the better formability.

Lubrication has twofold effects. Lubrication of the flange is beneficial since it reduces the work expended to overcome friction. However, it is necessary to keep the friction between contact surfaces of the punch and the metal sheet in deep drawing, because this friction force aids the draw of the metal into the die cavity [7]. One method to solve this problem is to apply non-uniform lubrication, i.e. different lubrication conditions at 
different areas of the blank before drawing [25]. By controlling the type, amount and distribution of lubricants, the flow of material can be controlled, which decreases strain localization and benefits the formability. However, this method is used rarely because of the complication of using different lubricants on the same blank.

Although lubricants are easy to use and have wide applications at both research apparatus and commercial industries, they are often environmentally objectionable, and their removal produces environmental and economic costs [1]. Currently, stampings are moving towards a lubricant-free operation so that lubrication may not be available to improve the formability in future.

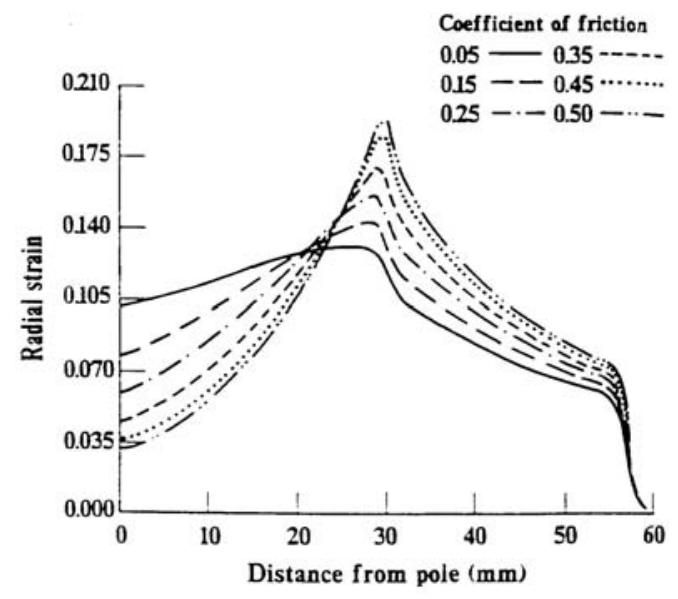

Figure 2.4 Friction effects on radial strain profiles along radial direction in simulating stretch forming behavior (punch travel distance $25 \mathrm{~mm}$ ) [13] 


\subsubsection{Varied blank holder force}

In a sheet metal stamping process of Figure 2.5 [27], the blank holder force is applied in a flat binder or in one with fixed height drawbeads to prevent wrinkling and to control the metal flow by friction between the sheet and the binder. If the blank holding force is not large enough, compressive stress tends to cause wrinkling of the flange and the unsupported side wall. But too high blank holding force produces unnecessary friction force, obstructs the metals from flowing into the die cavity and leads to metal sheet tearing. Therefore, blank holding force should be maintained in such a level that the maximum punch penetration is obtained while the wrinkling of flange is kept to a minimum [2, 25-27].

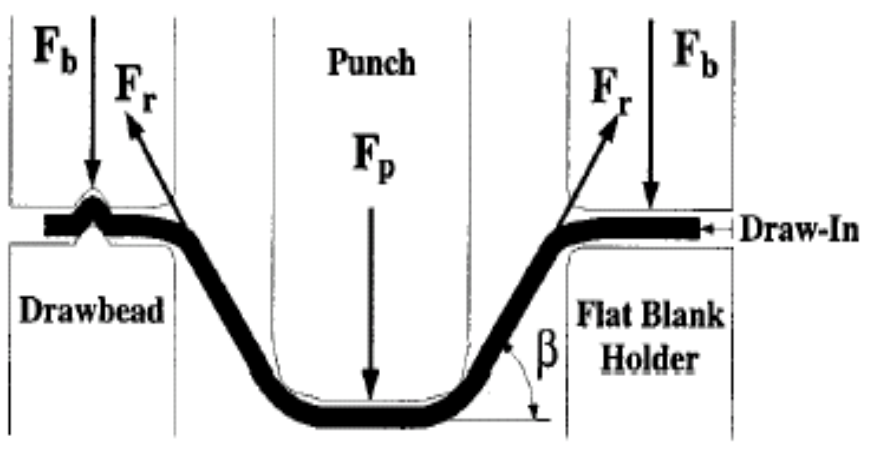

Figure 2.5 Schematic of a stamping process [18] $\mathrm{F}_{\mathrm{p}}$ : Punch force; $\mathrm{F}_{\mathrm{b}}$ : Blank holder force;

$\mathrm{F}_{\mathrm{r}}$ : Restraining force acting on the drawn blank

In conventional stamping, the blank holding force can be estimated by some empirical equations [28-30], but still need trial and error to adjust for actual process. S. Yossifon et 
al [26] performed a series of deep drawing tests with different constant blank holding force (BHF). They found that a different BHF leads to a different strain distribution and a different maximum punch force profile. The BHF should be within an acceptable range during the process to avoid wrinkling and tearing, which confirms the analysis of last paragraph.

Recently with the development of data acquisition systems and computer hardware, the technique of variable blank holding force has been investigated [27, 31-34]. This approach is reasonable since many process parameters vary during the stamping process, such as friction, flow stress and work piece shape. So the BHF should vary correspondingly during the process to better control the metal flow. Thiruvarudchelvan and Lewis [31] designed the die-holder-blank system so as to apply a blank holding force approximately proportional to the punch force during cup drawing and the new method is capable of producing a wrinkling-free part. Cao and Boyce [32] designed a single variable blank holding force trajectory for a conical cup forming by their FEM model. This trajectory was implemented in experiments and led to a $16 \%$ increase in the ultimate forming height of the cup over the traditional processes.

\subsubsection{Multi-point die-cushion technology}

Except varying BHF with time, investigators [25, 33-34, 37] designed the multi-point die-cushion technology to change the blankholder pressure distribution at the different positions of the blank holder. This technology provides accurate and flexible control means, because the strain states of blank flange may not be same at different areas of the 
flange. But to apply this technology is only possible under the condition that the blank holder can transmit the adjusted forces to the different area, which requires a new hydraulic press design or modifies existing presses.

With the new technology, Klaus-Jurgen Pahl [37] made a multi-point-control unit to provide different blankholder pressures at different locations of blank holder for production of bowl sink, shown in Figure 2.6. He successfully produced a complex bowl stainless steel kitchen sink which was hard to produce with conventional uniform blankholder pressure, shown in Figure 2.7 [37]. From Figure 2.7, it can be clearly seen that the corners and the straight sides of the sink have different trends of metal flows and hence different strain states. Therefore, different blankholder pressures should be applied at these areas to control the metal flows. 


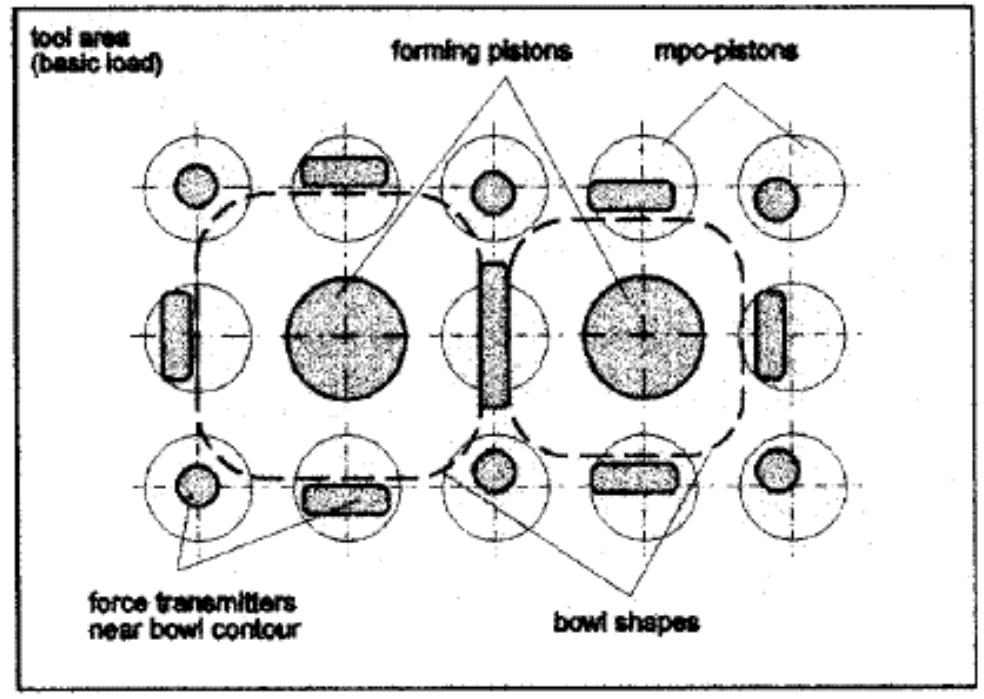

Figure 2.6 Multi-point-control unit for production of bowl kitchen sinks [37]

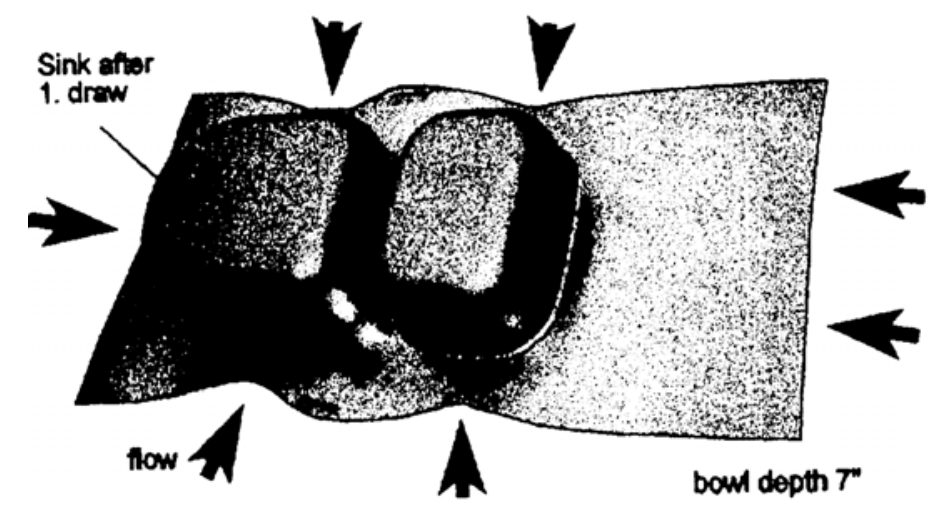

Figure 2.7 Drawing characteristics of a bowl stainless kitchen sink [37] 


\subsubsection{Active drawbeads}

The blankholder creates the restraining force by friction between the metal sheet and the blank holder. When a high restraining force is needed, higher blank holder pressures have to be applied to increase the friction force, which may cause the wear in the tooling and blank [2]. If the required blank holder pressure is too high, it may be beyond the capacity of the press. At such situation where a high restraining force is required, the use of drawbeads is necessary.

A drawbead is an insert or rib-like projection on the draw ring or blankholder surfaces that aids in controlling the rate of metal flow [2]. The drawbead increases the restraining forces by two components. One is caused by cyclically bending and unbending the metal sheet as it flows over the drawbead, and the other is from friction [7]. Thus, a drawbead can provide high restraining force at relatively low blankholder pressure [2]. But drawbeads severely deform the metal sheet passing through them, which may cause surface damage.

Fixed drawbeads have been used in sheet metal forming for a long time [2, 17]. By different heights of drawbeads and different locations at blankholder, it is possible to provide a differential control of metal flow at selected locations. These are the common ways used before, and the optimal height and location of drawbeads often are based on a procedure of trial and error [7, 28-29]. Recently the active drawbead concept is introduced and investigated by experiments and FEM simulations [2, 38-40]. The reason is the same as that of variable blank holding force, i.e. corresponding to the varying 
parameters throughout the process. The results showed that controlled drawbead height during process does improve the formability of sheet metal forming. Rui Li and Klaus J. Weinmann [38] studied the drawbeads' effect on formability of AA6111-T4 in nonsymmetric panel stamping. They found that the active drawbead case produces a successful part 7.69\% deeper than that obtained with flat binder and $16.7 \%$ deeper than that with fixed drawbeads. Furthermore, the optimal choice of the drawbead trajectory depends on the selection of the BHF. Their results demonstrated that the drawbead trajectory influences the strain distributions and improves the draw depth of the metal parts, shown as Figure 2.8 [38].

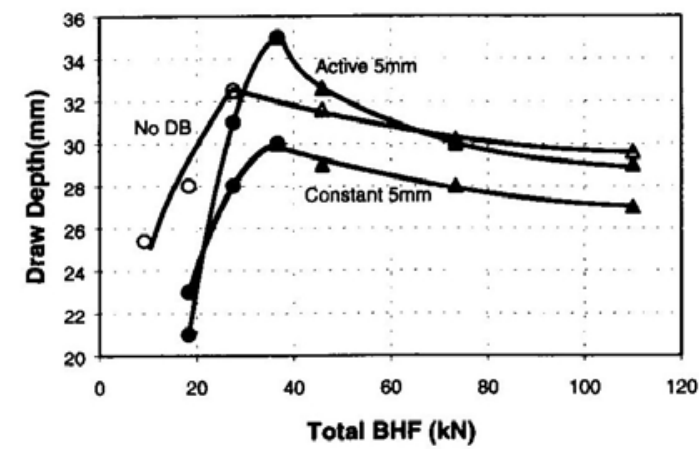

(a)

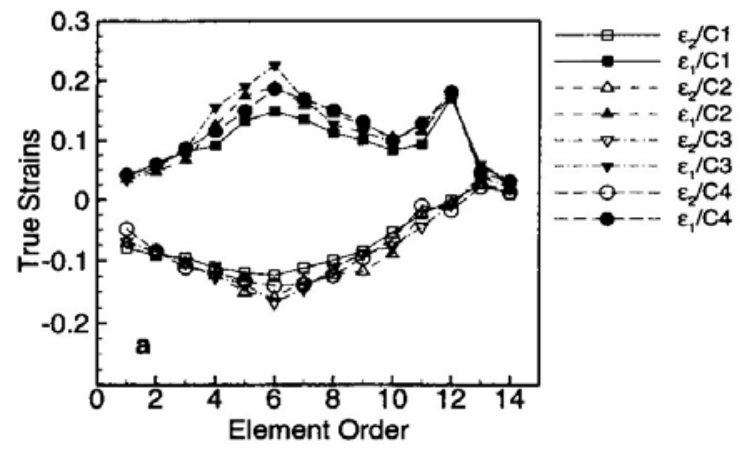

(b)

Figure 2.8 (a) Limit curves obtained for different drawbead trajectories; (b) Comparison of strain distributions between different drawbead trajectories [38]

Active blank holding force and drawbeads provide effective and flexible means to improve formability of sheet metal forming. There are already some applications of 
active blankholder force in commercial stamping presses [35, 37]. However, obviously they increase the complexity of the forming press and tooling system, and need accurate control system. Moreover, the design methodology of optimum trajectory needs much work to do.

\subsubsection{Initial blank design}

Since the final part is formed from the flat initial blank, the initial blank's size and shape definitely affect the metal flows of stamping. As shown in Figure 2.7 [37], it can be clearly seen that there are different tendencies of metal flows along the flange of the kitchen sink. At the corners of the flange, there exist redundant metals that cause compressive stress and resistance to draw into the die cavity. While at the straight sides, more metals are drawn into the die cavity. Therefore, if using an initial blank with round corners instead of these rectangular corners, there will be less redundant metal at the corners, which decreases the compressive stress and improves the resistance to wrinkling. And if there are more metals left at the positions of initial blank corresponding to the straight sides of final parts, there will be sufficient metals that can be drawn into the die cavity. The analysis above shows that changing initial blank shape does benefit the proper metal flow and hence improves the formability of sheet metal forming.

By FEM, R.J. Comstock Jr. et al simulated the cup drawing with different initial blank diameters [41]. They found that initial blank diameter affects the strain distribution and the drawn cup height. In their results, an initial blank diameter of $119 \mathrm{~mm}$ produced the maximum cup height while necking occurred early in the 122mm-diameter blank during 
drawing. Their results were in good agreement with their experiment results, which indicated that the initial blank size does affect the strain states in sheet stamping.

Designing optimal initial blank has been used for a long time in sheet metal stamping, since it is relatively simple to apply and does not increase the complexity of stamping process. Furthermore, optimal initial blank can reduce the cost of materials. However, the design of initial blank is not easy because of the complexity of materials behavior during the actual stamping process. There are many empirical rules to determine the optimal initial blank for different parts [28-29, 42]. Recently some different approaches by FEM simulation have been developed to design the optimal initial blank [43-47]. All of these methods need a guess of initial blank, get the final shape from this guess by FEM simulation and compare it with the desired final shape. According to the difference, the initial blank is modified to input again. The optimal initial blank is obtained after several such iterations. The results show the good potential to design the optimal initial blank. 


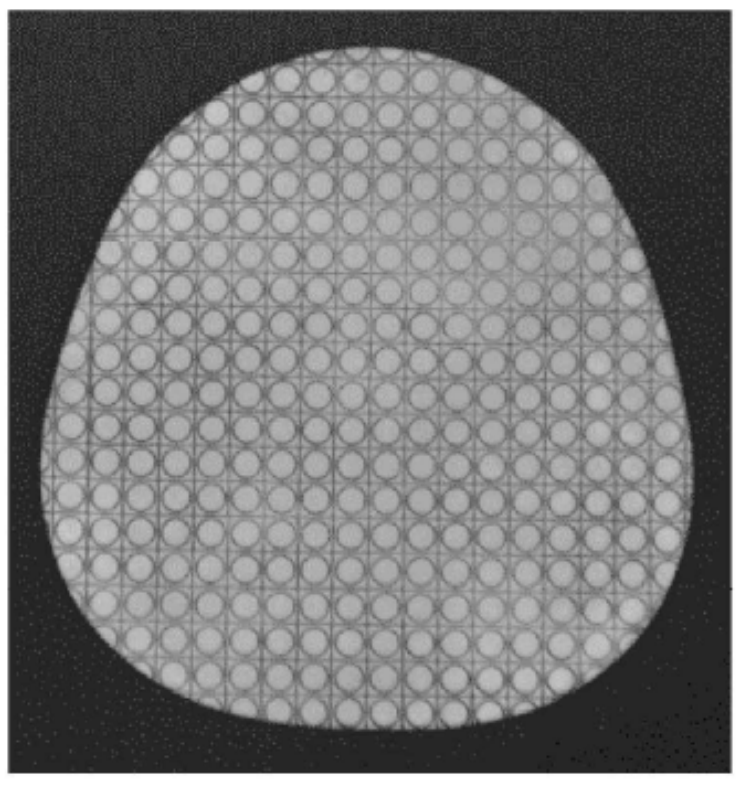

(a) optimal blank

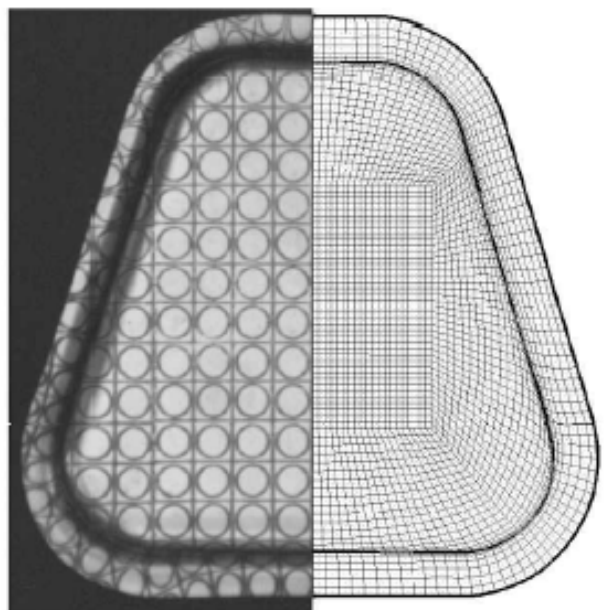

(b) after drawing

Figure 2.9 Experiment results for the optimal blank (trapezoidal cup) [46]

\subsubsection{Summary}

This review shows that the majority of the manufacture methods to improve formability focus on devising ways to control the material flow in order to affect the strain distribution. The methods described above all have drawbacks. Furthermore, no method could directly give the optimal solution to practical stamping process. All of them need trial and error to get an optimal solution.

Numerical simulation has been applied to optimize the sheet metal forming process [40, 48-50]. It saves the design time and tooling and material costs. Basically it requires the initial input parameters, relies on the iteration of the finite element model to gain an 
understanding of the effect of input changes, and then recommends system improvement [48]. The numerical simulation technology is still trial-and-error in fact.

\subsection{Electromagnetic forming}

\subsubsection{Fundamental of electromagnetic forming}

In principle, electromagnetic forming system consists of a capacitor bank, a conductive coil and the metallic workpiece to be deformed [1, 51-56]. The experimental scheme for flat sheet metal forming is shown in Figure 2.10 [1]. The capacitor bank is connected to the coil that is very near the metal sheet. When the main switch is closed, the rapid discharge of stored electrical energy from the capacitor bank provides a large current flow through the coil. The current produces a transient magnetic field around the coil that will induce eddy currents in the metal sheet [8]. The currents in the coil and metal sheet always travel in opposite directions. So there is always an electromagnetic repulsive force between coil and metal sheet, which will deform the metal sheet.

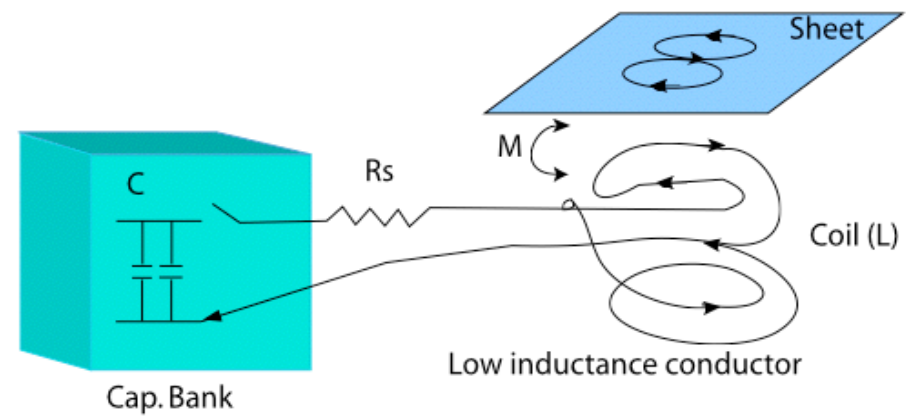

Figure 2.10 Schematic diagram of electromagnetic forming 
This process is governed by the classical coupled differential equations [57],

$$
\begin{aligned}
& \frac{d}{d t}\left(L_{1} I_{1}+M I_{2}\right)+R_{1} I_{1}+\frac{Q_{1}}{C_{1}}=0 \\
& \frac{d}{d t}\left(L_{2} I_{2}+M I_{1}\right)+R_{2} I_{2}=0
\end{aligned}
$$

where $L_{1}, R_{1}$ and $C_{1}$ are the inductance, resistance and capacitance of the capacitor bank and coil. $L_{2}$ and $R_{2}$ are the inductance and resistance of the metal sheet. $M$ is the mutual inductance between the coil and metal sheet. $Q_{1}$ is the stored charge in the capacitor bank. $I_{1}$ and $I_{2}$ are currents in the coil and metal sheet. The typical current traces of $I_{1}$ and $I_{2}$ are shown at Figure 2.11.

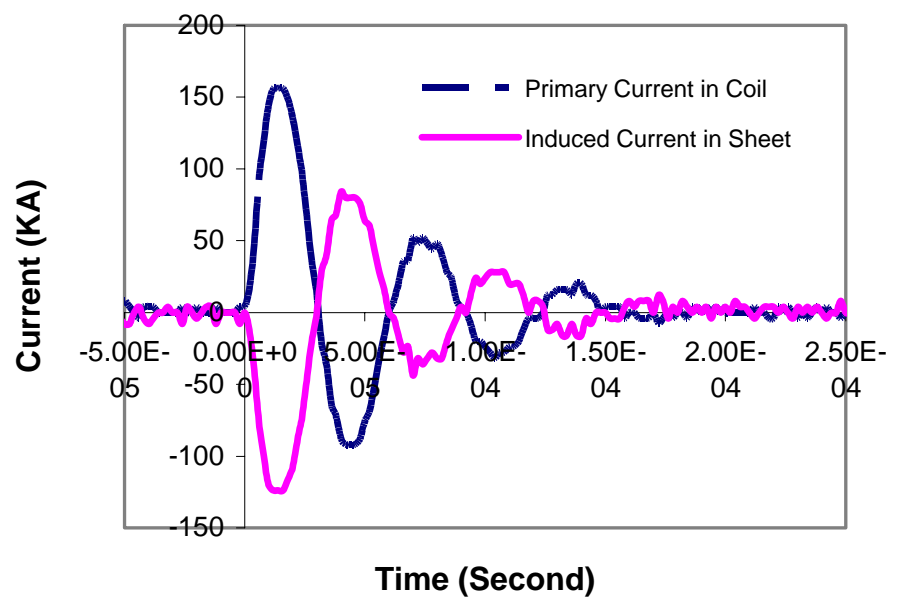

Figure 2.11 The typical traces of primary and induced currents 
The electromagnetic force per unit length that acts between the actuator and metal sheet can be approximated as [59],

$$
\frac{F}{l}=\frac{\mu_{0} I_{1} I_{2}}{2 \pi d}
$$

where $d$ is the distance between the metal sheet and the coil. Required electromagnetic forces can be easily obtained to exceed the plastic yielding of metal sheets, and provide material acceleration to high velocity by adjusting the stored charge in the capacitor bank [60-61]. Moreover, the electromagnetic force distribution can be directly controlled by the spatial configuration of the coil, since the electromagnetic force is only significant the area close to the coil.

Although the Eq. (2.3) gives an estimate about the electromagnetic forces per unit length, it is difficult to calculate the accurate electromagnetic forces during the process. Because many aspects are coupled and vary with the moving of metal sheet, detailed calculations are very complex [53-61]. Figure 2.12 shows that the initially flat metal sheet is moving away from the beginning of the electromagnetic forming [6]. Actually many researchers have applied the finite element simulation for electromagnetic forming [62-68]. It is only possible to get very accurate solution for electromagnetic forming by numerical simulation. 


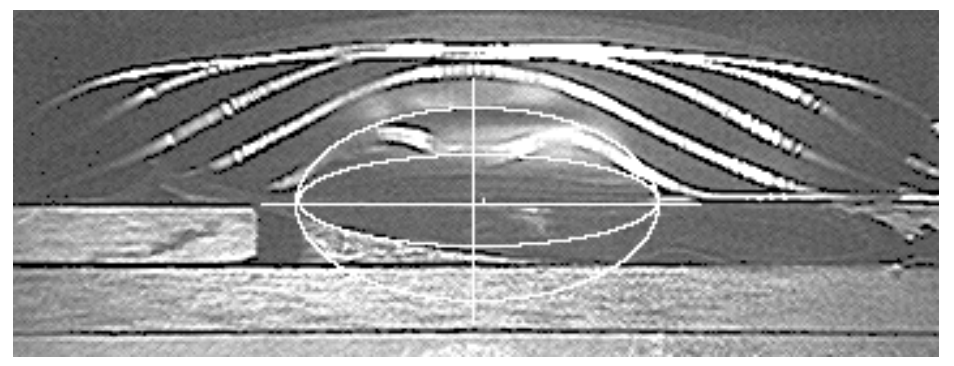

Figure 2.12 Typical images of electromagnetic forming process (Vave. $=200 \mathrm{~m} / \mathrm{sec}$, 50E-6 sec between images) [6]

\subsubsection{Some important features of electromagnetic forming}

Some important features make electromagnetic forming different from other forming processes. They must be considered when we apply electromagnetic forming. Below a brief introduction will be given.

(1) Metal sheet in electromagnetic forming must be conductive enough to allow sufficient induced eddy currents. The efficiency of electromagnetic forming is directly related to the conductivity of the metal sheet. Metals with poor conductivity can only be effectively formed if an auxiliary driver sheet with high conductivity is used to push the metal sheet $[35,36]$. Aluminum alloys are good conductors with higher conductivity than plain carbon steel [43]. So aluminum alloys are more suitable for electromagnetic forming.

(2) The discharge time of electromagnetic forming is very short, generally of the order of 10 microseconds [6]. The conventional sheet stamping usually takes a few seconds. 
Moreover, Figure 2.11 shows that the currents in coil and metal sheet are damped sinusoidally, and most deformation work is done within the first half cycle. Therefore, electromagnetic forming can be considered as a transient event compared with conventional sheet stamping.

(3) As mentioned before, the distribution of electromagnetic pressure can be directly controlled by the spatial configuration of the coil. And the magnitude of electromagnetic pressure can be controlled by the stored charge in the capacitor bank. So electromagnetic forming is easy to apply and control, making it very suitable to be combined with conventional sheet stamping. The practical coil can be designed to deal with the different requirements of each forming operation.

\subsubsection{Benefits of electromagnetic forming}

Electromagnetic forming usually is used to accelerate the metal sheet at velocities up to a few hundred meters per second, which are 100 to 1000 times greater than the deformation rates of conventional quasi-static forming such as the sheet metal stamping $(\sim 0.1 \mathrm{~m} / \mathrm{s}$ to $\sim 100 \mathrm{~m} / \mathrm{s}$ ) [6]. It is well known that high deformation velocity (over about $50 \mathrm{~m} / \mathrm{s}$ ) can significantly increase the formability of metals by several times, compared with those obtained in conventional quasi-static forming [51-56, 70-72]. Figure 2.13 shows the results of some experiments in high velocity forming of aluminum alloys presented in FLD data format [70]. The extended formability is available over a broad range of deformation velocities, which is kind of material dependent but generally lies over $50 \mathrm{~m} / \mathrm{s}$ [53]. 


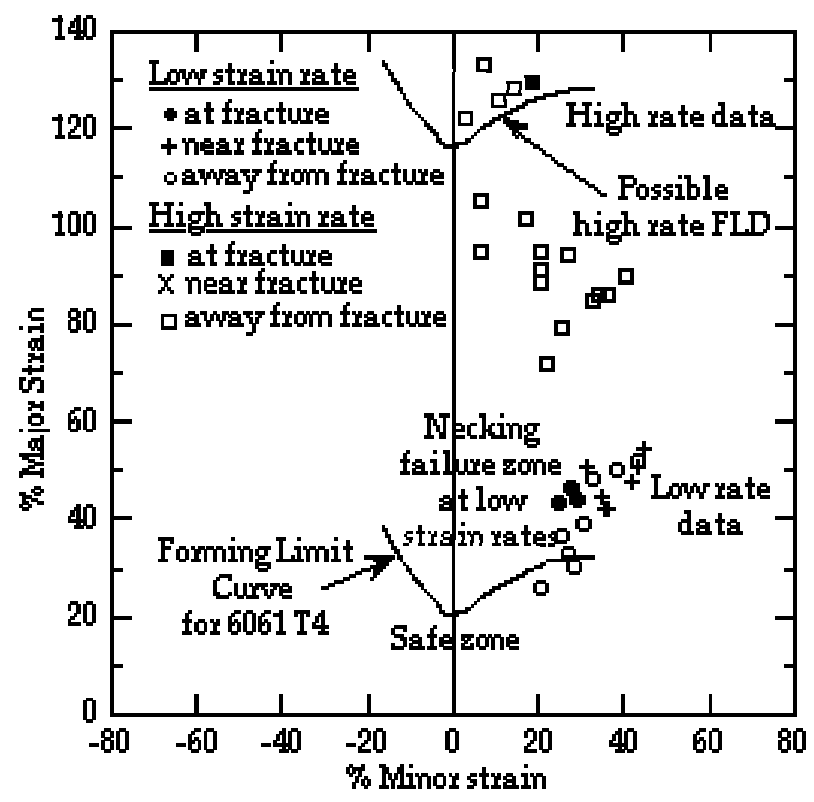

Figure 2.13 Forming limit diagram with high velocity forming data [53]

A complete understanding of how formability is affected by high deformation velocity is still lacking. However, some issues about the improvement of formability are clear now and will be briefly discussed.

The effect of inertia on a neck is the most straightforward way to explain the improved formability in high velocity forming. Several researchers have shown that failure in a tensile sample is delayed when inertial forces are relatively large [72-77]. Hu and Daehn believed that the velocity gradient in the necking area leads to non-uniform inertia forces [72]. And the inertia forces produce the additional tensile stress and strain at the areas outside of necking. 
The second reason for improved formability is the inertial ironing. The sheet metal with high velocity impacts the stationary hard die, and produces a very large through-thickness compressive stress. This is termed as inertial ironing [53]. This compressive stress can be much larger than the metal flow stress, and hence should have effect on the deformation modes.

There are some other issues having effects on the formability in high velocity forming, such as boundary conditions, constitutive equation changes at high velocity [72-77].

\subsubsection{Applications of electromagnetic forming}

Electromagnetic forming is a process that has been applied since the 1960's, but has not very extensive use [6, 53]. Its common application is to expand or compress axisymmetric metal parts. It has been commercially applied for long time for the joining and assembly of concentric parts and compression crimp seal [55]. Figure 2.14(a)-(c) show schematics of the general classes of electromagnetic forming coils and metal workpieces [78]. 

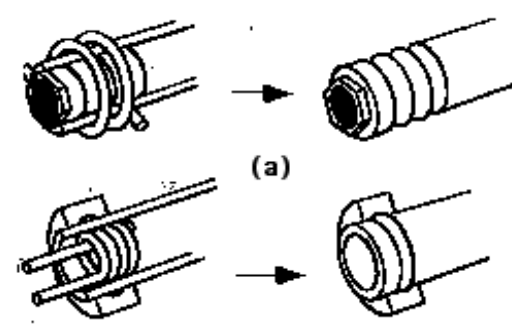

(b)

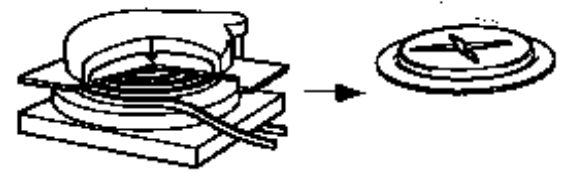

(c)

Figure 2.14 Electromagnetic forming application [78]: (a) tube compression; (b) tube expansion; (c) sheet or pan forming

Since electromagnetic forming provides extended forming limits with high deformation velocity and there are needs to improve the formability in sheet metal forming, Vohnout and Daehn [6] proposed Matched Tool-Electromagnetic Hybrid Sheet Forming (MTEM), shown in Figure 2.15. Generally the stamping parts are large and need very large EM energy input if using high velocity forming to form the whole metal parts alone. Due to the practical limits of strength of tooling materials and the capacity of capacitor bank, the electromagnetic forces cannot be made arbitrarily large to form large parts. To reduce the requirements of the robust tooling and the large capacitor bank, the high power pulses will be applied only in the final forming and only at the local areas of the part where required. And the most of the parts will still be formed by conventional stamping. This is the key idea of MT-EM process [6,53]. So in this process, the parts are pre-formed to 
some optimum extent by the conventional stamping of the matched tooling. Final forming of tight corners, sharper details and sizing would be accomplished by electromagnetic repulsion forces generated at the required areas of the part by a set of electromagnetic coils embedded in the tool halves [6, 53].

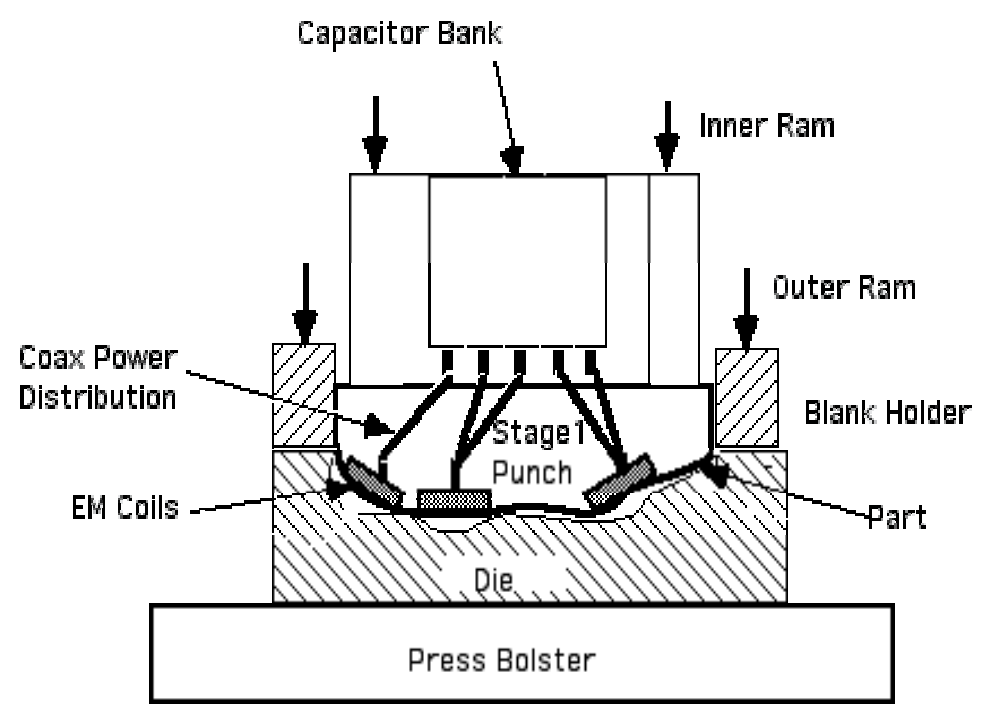

Figure 2.15 Matched tool-electromagnetic hybrid sheet forming [6]

Using this MT-EH process, Vohnout carried out a demonstration to form an aluminum alloy door inner, which could not be fabricated without failure by conventional stamping, shown as Figure 2.16 [6]. The door panel was formed close to the desired shape with conventional stamping first. Then a secondary electromagnetic operation was carried out to form a sharp corner by the designed coils embedded in the conventional tools. The results were good and shown as Figure 2.17 [79], and the electromagnetically reformed 
corner was very close to the desired shape. Plane strain values in excess of $25 \%$ were observed on the electromagnetically reformed panel, which are larger than those in conventional stamping [6].

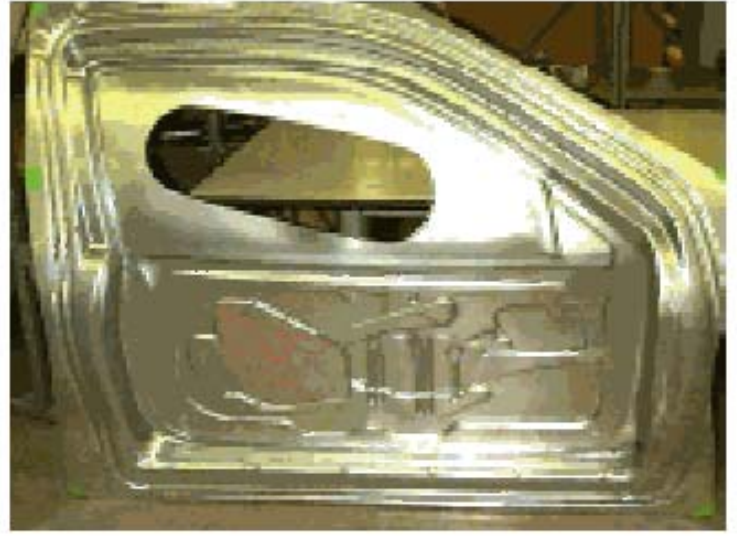

Panel A, production steel

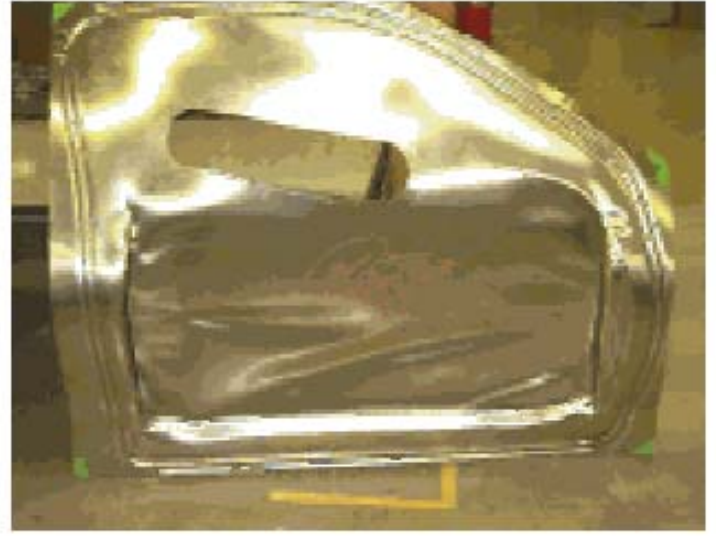

Panel B, 6111-T4 aluminum

Figure 2.16 Automotive inner-door panels, forming demonstration [6]
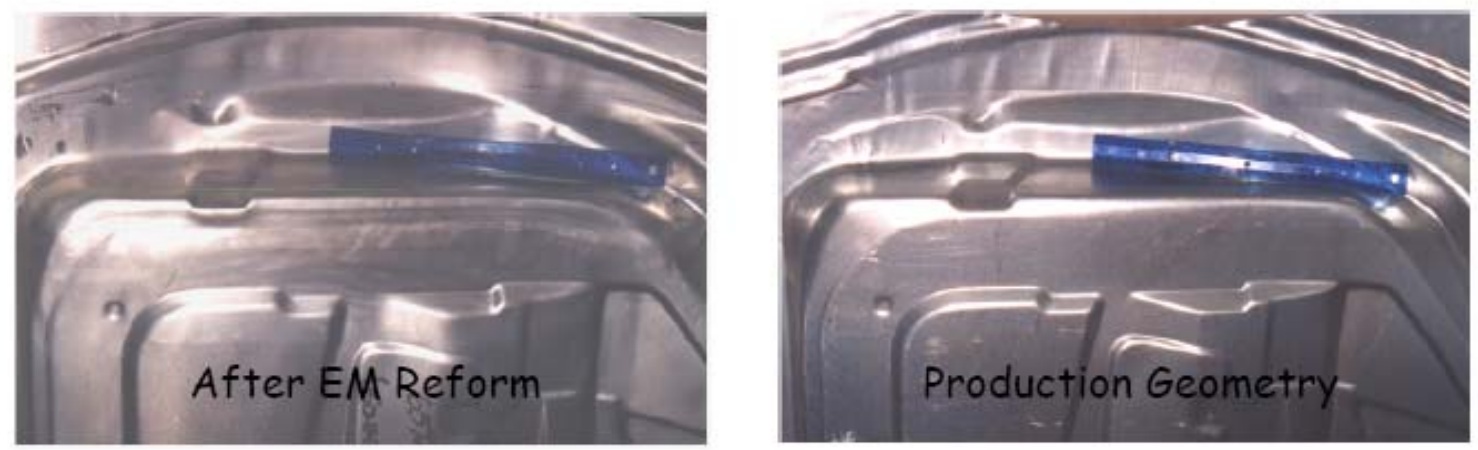

Figure 2.17 The electromagnetic re-forming process carried out on the door panel [79] 
This experiment is significant since it proves the feasibility of the incorporation of electromagnetic coils with the conventional stamping tools. However, it also shows some weaknesses. First, when producing high deformation velocity over a large area, a large energy pulse needs to be provided from the capacitor bank. Coil design and construction will be difficult, and robust tooling is required to resist shock. Another problem is that it is not easy to precisely control the final shape at high velocity. In this experiment the final shape is very close to the desired shape, but still needs a calibration operation [53].

\subsection{Summary}

The principal goal of this dissertation is to develop a new technology to improve the formability of sheet metal stamping. For a given material and given shape of part, many manufacturing method have been developed to improve the formability.

The first half of this chapter reviewed several methods, which are used to control metal flow and then affect the strain distributions. These methods can improve the formability of stamping but still have their individual weaknesses. The second half of this chapter introduced the electromagnetic forming and the application on sheet metal forming. MTEM process was developed to form the metal parts by EM high velocity forming and to improve the forming limits. This method achieved high forming strain but required high forming energy and robust tools.

Therefore, could we use many small EM pulses to improve the formability of stamping instead of single large EM pulse? Small EM pulses may not produce high deformation 
velocity to improve the forming limits of metals. However, as described before, formability of stamping can be improved by controlling the strain distributions. So a new approach, electromagnetically assisted sheet metal stamping, is proposed, which will be discussed in next chapter. In this new approach, electromagnetic forces are used to control strain distribution, rather than to produce very high deformation velocity. 


\section{CHAPTER 3}

\section{ELEMENTS OF ELECTROMAGNETICALLY ASSISTED SHEET METAL STAMPING}

\subsection{Electromagnetically assisted sheet metal stamping}

Electromagnetically Assisted Sheet Metal Stamping (EASMS) is to apply appropriate small electromagnetic pulses to the strategically chosen regions of the metal sheet in order to control the strain distribution. The key idea of this approach is to embed the electromagnetic coils into the suitable position of conventional tools and directly stretch the area with little or small strain state by electromagnetic forces. The conventional tools form the contour of the desired shape, while electromagnetic forces control the strain distribution to improve the deformation and to avoid undesired large strain states. In this way, a more uniform strain distribution can be achieved, and large strain states that lead to failure can be avoided. But where and how to apply the electromagnetic forces need to be carefully considered.

\subsubsection{Strain states of sheet metal stamping}

Sheet metal stamping is a non-steady-state process in which the stress and strain states change significantly during the operation. And the deformation is not homogeneous in stamping, and therefore the stress and strain states are quite different at various locations 
of the metal part. Figure 3.1 shows the different strain states in a typical part produced by stamping process [5]. Basically, there are three different areas in a typical part during stamping. The first one is the flange area which is drawn into the die cavity and usually has small strain states, such as E-D and J-H in Figure 3.1. Wrinkling is likely to happen here. The second one is the wall area which transmits the force to the flange area and has relative large strain states, such as D-C-B and H-G-F in Figure 3.1. Tearing often occurs here. The last one is the bottom area which is below the punch face and has relative small strain states because of the friction between punch and blank, such as B-A and F-A in Figure 3.1 [5]. Therefore, the best areas which electromagnetic forces could be applied are the flange area and the bottom area. And electromagnetic coils could be embedded into the flange surface of die and the bottom of punch to increase the stretch and draw-in.

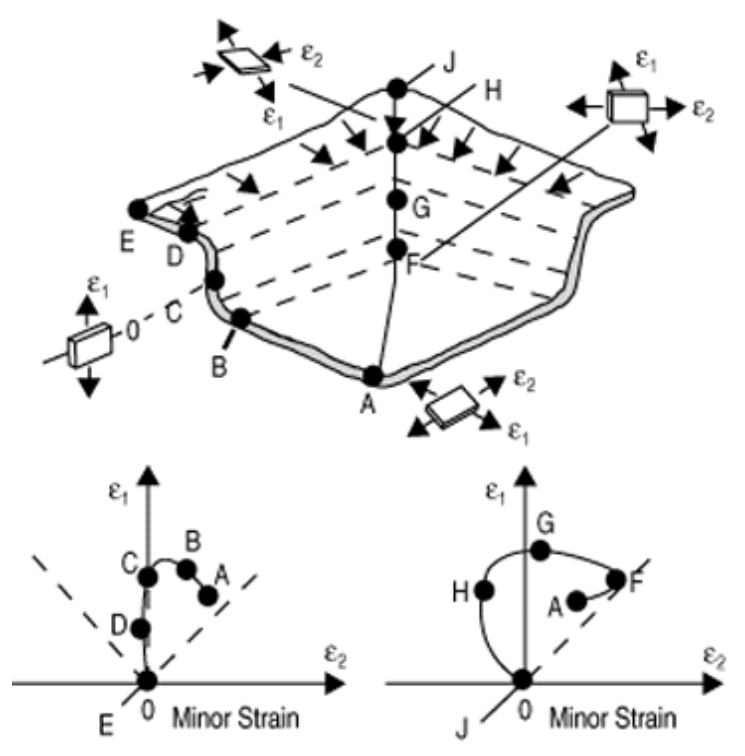

Figure 3.1 Schematic of strain states in a stamped metal part [5] 


\subsubsection{General application of EASMS}

According to above analysis, possible positions of electromagnetic coil are the flange surface of the die and the bottom of punch. Figure 3.2 shows the schematic of electromagnetically assisted sheet metal stamping [1]. The geometry and exact position of the coils should be decided according to the specific stamping process.

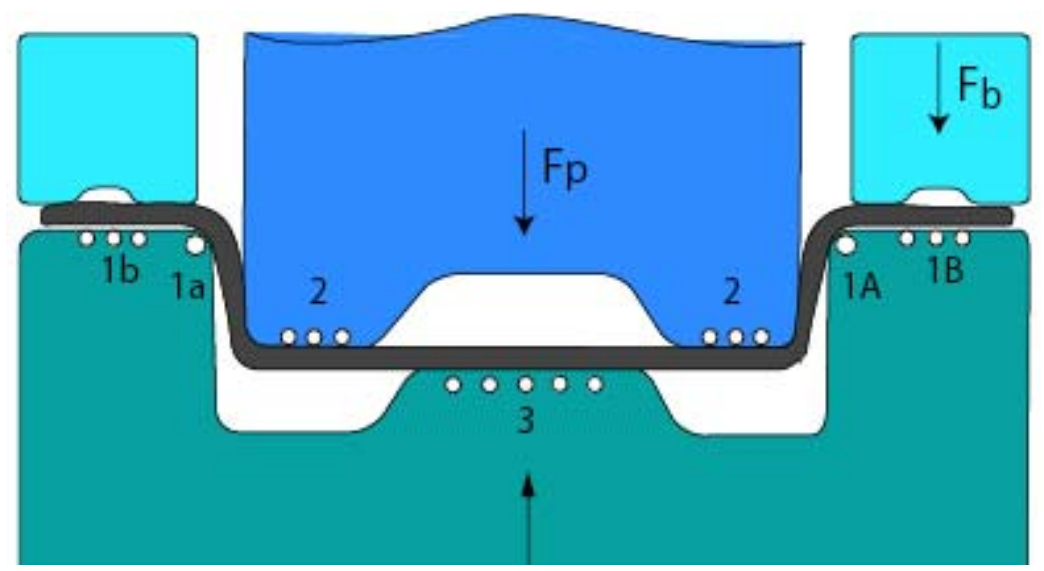

Figure 3.2 Schematic of electromagnetically assisted sheet metal forming [1]

After one or all coils are embedded into the conventional tools, they are coupled with a capacitor bank. During stamping process, small energy pulses are given to the coils from the capacitor bank. In each pulse, the repulsive electromagnetic forces between coils and metal sheet deform the metal blank, and make a stretched "bubble" above coils. This stretched "bubble" causes plastic extension in the plane of the sheet and the unbalance of forces over the blank. After each pulse, punch continues to advance into the die cavity. The stretched "bubble" area will be pulled out towards the die cavity along the tool 
surface because of the unbalance of forces, and re-conformed to the tool surface. Meanwhile, the plastic extension of "bubble" area provides the increase of line length to create the part.

In each pulse, besides stretching, the repulsive electromagnetic forces also can draw more metals in from the flange area. In this way, the regions with little or small strain state are deformed. And the extra line length produced by electromagnetic forces is converted towards the die cavity, which leads to the improvement of formability of stamping process.

The magnitude of repulsive electromagnetic pressures can be easily adjusted, and exceed the yielding strength of the sheet metal by several times, by controlling the electrical energy stored in the capacitor bank. It is easy to control the magnitude of plastic deformation caused by EM pressures. Besides, the spatial distribution of electromagnetic pressures is directly controlled by the geometry of coils. By careful design of the geometry of coils, it is also easy to put the electromagnetic pressures where needed. Therefore, EM pressures can be applied easily to the positions needed with appropriate magnitude, which makes it possible to deform the sheet at the position needed with suitable deformation level.

The general operation procedure of electromagnetically assisted sheet metal stamping is as following: 
(1) The blank holder clamps the blank flange, and then punch advances to draw metals into die cavity;

(2) At a predetermined punch displacement, a small energy pulse is given to the embedded coils and a stretched area is formed above the coils;

(3) Punch continues to advance. More metals are drawn into the die cavity and the stretched area is pulled out.

(4) Repeat steps (2) and (3), until the desired shape is formed;

After each pulse, punch load is reduced when punch continues to advance, because of the unbalance of forces caused by the stretched "bubble" area. And the punch load will increase with the stroke of punch until the next electromagnetic pulse makes another stretched "bubble" area.

It seems that EASMS has low production rate and long cycle time because of the many times of EM pulses. This problem can be solved by the advanced control technology of capacitor banks. The EM pulse is very short, generally of the order of 10 microseconds. Comparing to the several seconds of conventional stamping, it can be considered as a transient event. Therefore, the electromagnetic forming can still happen when the punch is moving. It is not difficult to cycle a capacitor bank many times in a second or less. Typical commercial capacitor bank systems have on the order of 10 capacitors that are individually switched, and all switches are usually closed simultaneously. It is a simple matter to charge all the capacitors while loading a part and then close the switches for the capacitors (all connected to a common bus line) sequentially as the punch moves over a 
time period that may be a small fraction of a second. There are no fundamental reasons that this cannot accommodate a total part-to-part cycle time on the order of 5 or less seconds (as is typical in auto body panel stamping) [1].

\subsection{Engineering design and optimization for EASMS}

The engineering design for EASMS is complex and needs to optimize. It is impossible to provide a simple method to solve all the stamping processes. There are some researches about the optimization of conventional stamping [48, 81-85]. Most of them involved the finite element modeling and analytical modeling to understand the effect of processing and to predict the effect of input changes. According to the prediction, necessary changes of input can be made to avoid failure.

EASMS design is more complex than the conventional stamping, since there involves the electromagnetic forming. Basically the design for EASMS has five steps.

(1) The design should begin with the analysis of the conventional stamping, and find out the causes for the failures and the strain distribution of the parts.

(2) According to the analysis, the geometry of electromagnetic coils and the positions of the coils in the stamping tools should be first decided, i.e. where to apply the electromagnetic forces. There are two basic rules for this step. First, electromagnetic forces are mainly applied to increase the deformation of metal sheet. Hence, electromagnetic coils should be put close to the regions with littlie or small strain state. Second, electromagnetic coils should be very close to the metal sheet but can not contact the metal sheet during the whole stamping process. Electromagnetic coils 
near the metal sheet will make the electromagnetic forces stretch the metal sheet effectively. But contacting the electromagnetic coils will damage the coil insulation and lead to sparking.

(3) After the geometry and position design, the energy levels of electromagnetic pulses should be determined, i.e. how much to apply the electromagnetic forces. Basically EM energy level should not be too large and too small. Large EM energy makes large deformation, thins the metal sheet and reduces the life of EM coil. Small EM energy decreases the production rate and makes the cycle time longer. Generally EM energy level should be enough to make $5 \% \sim 10 \%$ stretching strain.

(4) According to the EM energy levels, the EM pulse schedule can be determined, i.e. when and how many to apply the electromagnetic forces. There are two methods to determine the EM pulse schedule. One is to estimate the strains caused by EM pulse and calculate the extra line length due to those strains. The punch advance depth between EM pulses should not exceed the value of the extra line length. This method is kind of complex. The second method is to determine the EM pulse schedule according to the punch load. The punch load increases with the punch advancement after every EM pulse. The EM pulse should be applied when the punch load increase to be at least higher than the punch load before previous EM pulse. The later one needs trial-and-error.

(5) Optimization of the process can be reached by iteration of previous steps until the desired shape is formed. 
For each design, analytical models can give a rough design and prediction. Simulation of eddy current and electromagnetic field in Maxwell 2D or other software can be used to predict the spatial distribution of electromagnetic forces and decide the geometry of the coils. And finite element simulation of the stamping process with any commercial FEM software package can be used to fine the parameters of electromagnetic pulse design. The analytical and FE models provide a comprehensive tool set for understanding and successful application of electromagnetically assisted sheet metal stamping. 


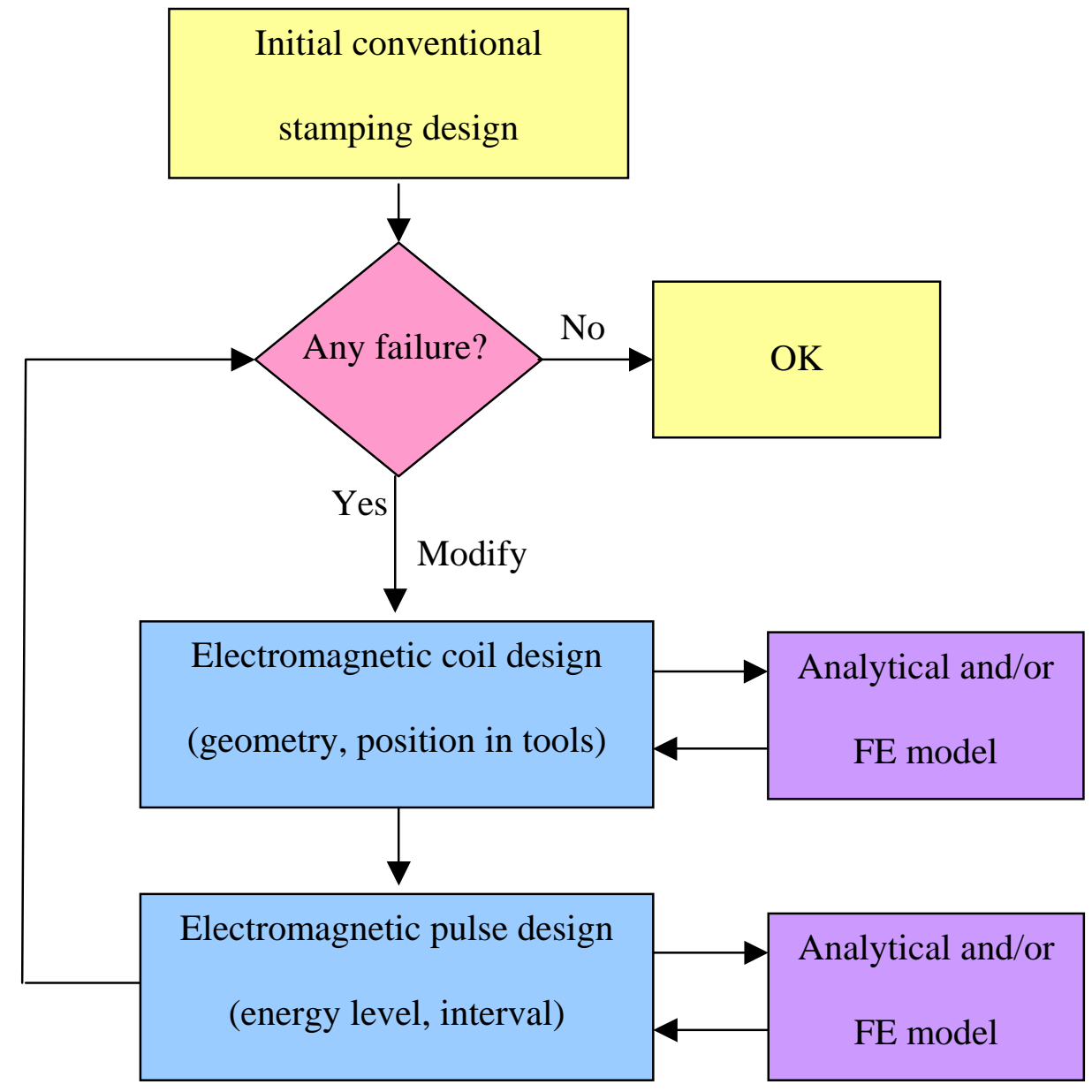

Figure 3.3 Design and optimization approach for electromagnetically assisted sheet metal stamping 


\section{CHAPTER 4}

\section{APPLICATION OF ELECTROMAGNETIC FORMING AT FLANGE IN DEEP DRAWING}

As discussed in last chapter, the possible positions for electromagnetic coil are the flange surface of the die and the bottom of punch. In this chapter, the effect of electromagnetic coil in flange will be investigated.

In stamping process, there are two kinds of flange: flange of straight side wall and flange of corner. There is no need to put the electromagnetic coil at the flange of straight side wall. The material in this kind of flange moves towards the die radius, bends to the curvature of the die radius, flows over the die radius and finally straightens again as it becomes parts of the box wall. During stamping, the resistance force to this kind of flange is from the friction and the bend-and-straighten. Actually, draw bead is often put in this kind of flange to limit the metal flow.

The flange of corner is different. When the material in the flange of corner moves towards the die radius, there is resistance force caused by compressing the larger blank circumference down to the smaller one. This resistance force increases with the punch stroke, and becomes the major force to limit materials draw in from the flange into die 
cavity. Axisymmetric deep drawing has the typical flange of corner, and is simple to study.

Therefore, electromagnetic coil was applied to the flange of axisymmetric deep drawing, in order to encourage the draw-in from the flange area. This chapter will begin with introduction of the experimental setup, then present the experiment results and discussions, and end with 2D theoretical analysis.

\subsection{Experimental setup}

\subsubsection{Initial analysis of deep drawing}

Axisymmetric deep drawing is relative simple comparing to other stamping process. An initially flat round blank is drawn by a cylindrical punch to form a cylindrical cup. Basically there are three regions for the metal part, the bottom region below the punch, the wall region in the die cavity and the flange region. The wall region has the largest deformation and the failure often occurs in the area just near the punch nose. The bottom region has small stretching because of the friction between the punch and the metal sheet. And the flange region should be pressed to prevent wrinkling. For a successful deep drawing, the flange region should be completely drawn into die cavity and converted into the wall region.

For deep drawing, the initial blank size is important. Suitable blank size can make the deep drawing successful and the flange region can be completely converted into the wall 
region. The large initial blank size causes early failure at the area near punch nose, because the wall region has not enough strength to draw the flange into the die cavity. Figure 4.1 is the contour of the maximum principal strain in plane for a sheet metal deep drawing with large initial blank size. It shows that the flange region and the bottom region have some strain level. To increase the stretching of the flange region, electromagnetic coils should be applied at the flange region of the die. The goal of the electromagnetic coils is to draw more flange region into the die cavity, convert more flange region to the wall region and hence improve the formability of deep drawing. The application of electromagnetic coil on the bottom region will be introduced in Chapter 6 .

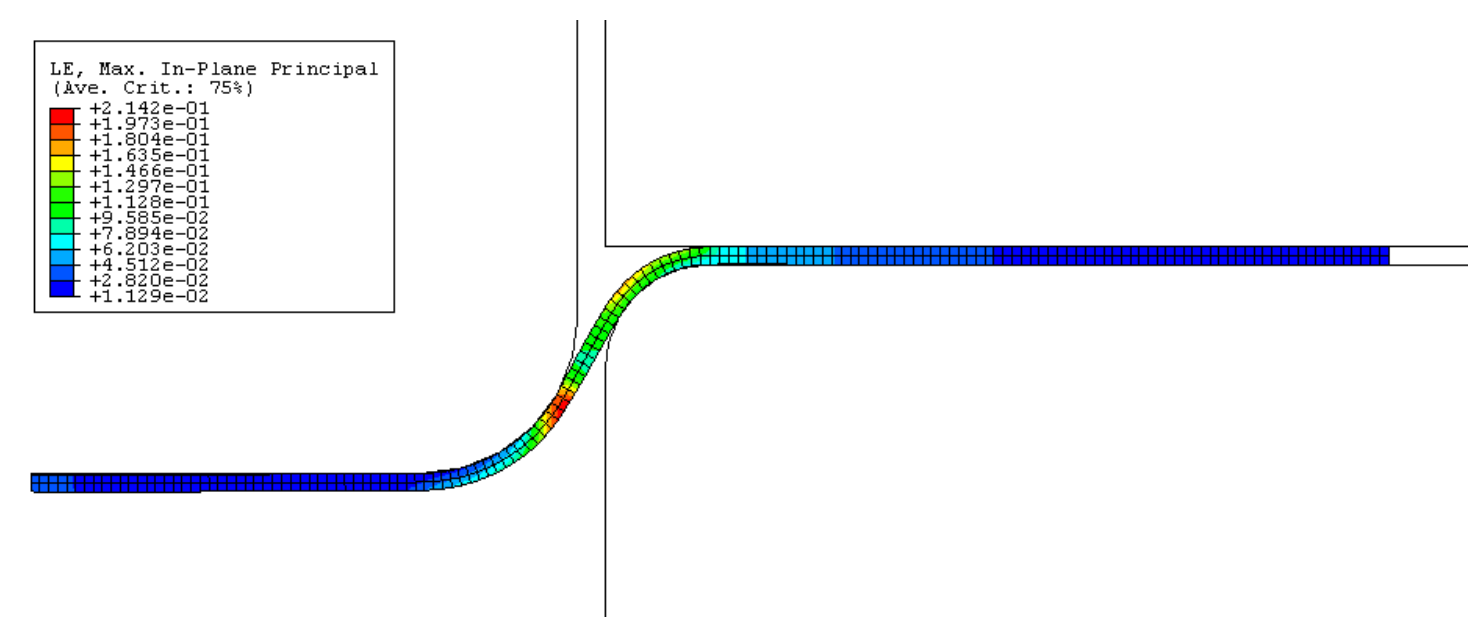

Figure 4.1 Contour of the maximum principal strain in plane for a sheet metal stamping with large initial blank size 


\subsubsection{Deep drawing tool}

The conventional axisymmetric deep drawing was carried out with the standard Swift cup test [80]. To compare the effect of electromagnetic forming, the electromagnetically assisted deep drawing was conducted with same tool sets, which are shown in Figure 4.2 and Figure 4.3. The tool geometric parameters are given in Table 4.1. The only differences between them are the electromagnetic coil embedded in the die and the hollow groove on the blank holder, which provides the electromagnetic stretching on the flange region.

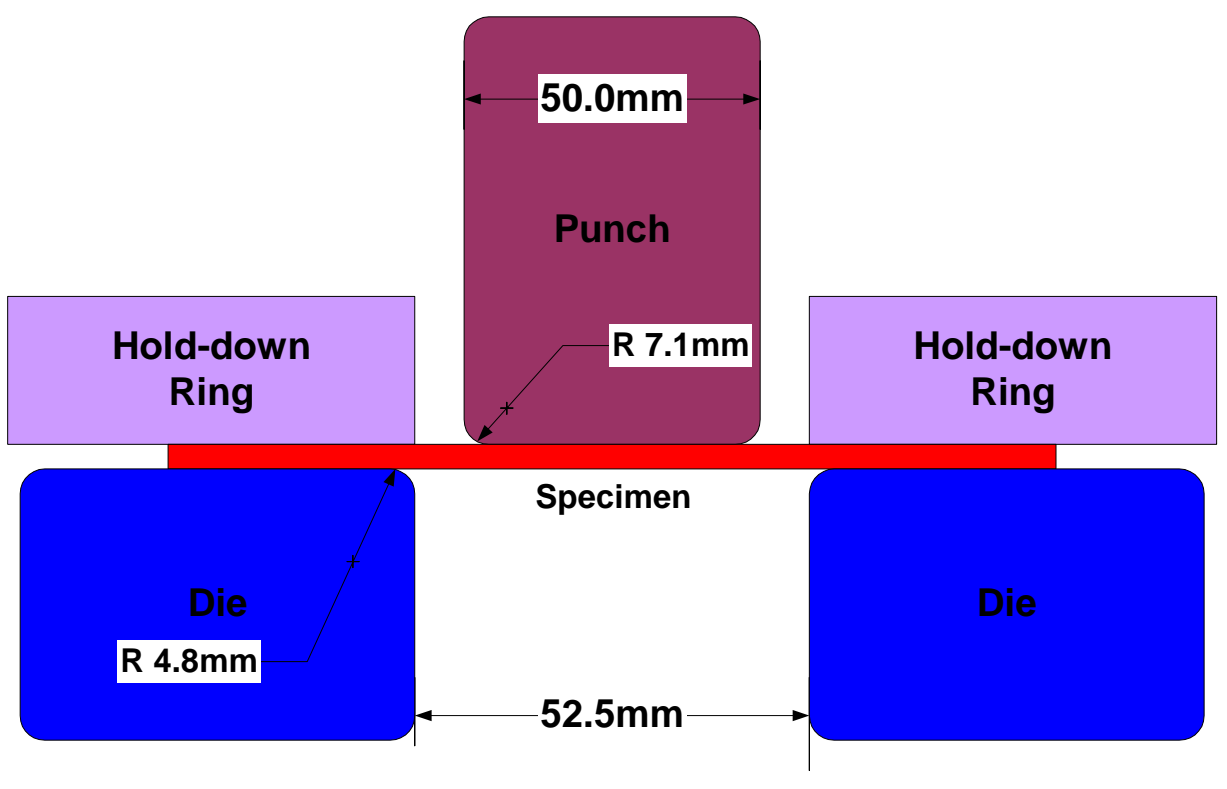

Figure 4.2 Conventional axisymmetric deep drawing 


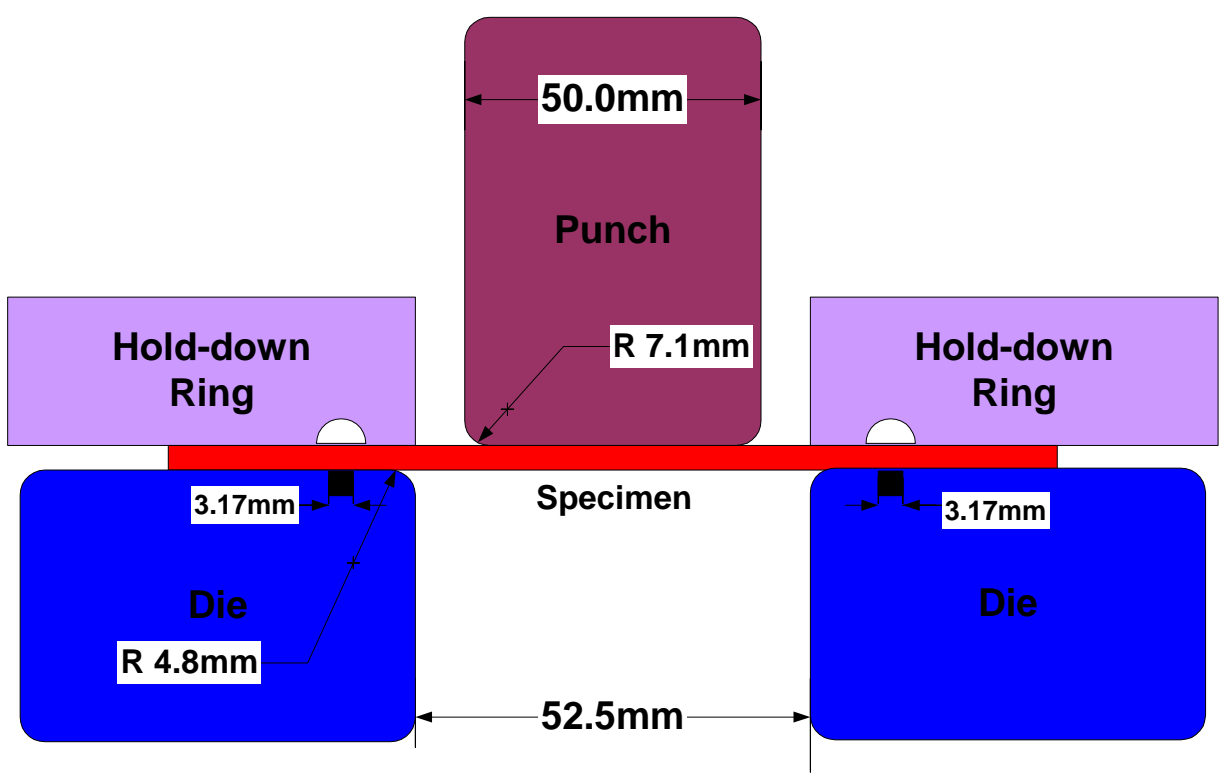

Figure 4.3 Electromagnetically assisted deep drawing

\begin{tabular}{cccc}
\hline Punch diameter & Die diameter & Punch profile radius & Die profile radius \\
\hline $50.0 \mathrm{~mm}$ & $52.5 \mathrm{~mm}$ & $7.1 \mathrm{~mm}$ & $4.8 \mathrm{~mm}$ \\
\hline
\end{tabular}

Table 4.1 Deep drawing tool geometric parameters

\subsubsection{Electromagnetic coil}

The geometry of electromagnetic coil for deep drawing is annular since the flange region is axisymmetric. And the cross section of the electromagnetic coil is rectangular. The height of the coil should be the long side, which will provide more strength to endure the repulsive electromagnetic forces between the metal sheet and the coil. Here the width of the coil was set as the half of the coil height. 
Because the flange region must pass the die profile before drawn into the die cavity, the position of the electromagnetic coil should be close to the die profile. In this way, more flange region can be affected by the electromagnetic forces. While, electromagnetic coil also should not be too close to the die profile. Since the flange region has the tendency to wrinkle and the metal around the die profile has no through-thickness pressure, there should be a gap between the electromagnetic coil and the die profile for the blank holder to apply the pressure to prevent the wrinkling.

There must be a hollow groove on the blank holder right above the electromagnetic coil to provide space for electromagnetic forming. Its width was set as twice of the coil width. And its height was set same as its width. To make the metal flow easy, the hollow groove should have the round edges. The important issue is that the width of the electromagnetic coil and the hollow groove should not be too big since there is no holding pressure from the blank holder on this area to prevent wrinkling. Here the width of the coil was set as $3.17 \mathrm{~mm}$.

The electromagnetic coil embedded in the die is shown in Figure 4.4. Figure 4.5 is the photograph of actual experiment setup. The electromagnetic coil was connected to the 48KJ capacitor bank by a coaxial coil. The $48 \mathrm{KJ}$ capacitor bank is the Maxwell Magneform capacitor bank, shown in Figure 4.6. The electromagnetic coil was cut by Water jet. Its material is aluminum alloy AA6061. In order to insulate the electromagnetic coil, the die and the blank holder were all made of G10. Besides, the 
electromagnetic coil should also be insulated with electrical insulation tape, such as Kapton tape. The geometric parameters of the coil and the hollow groove of the blank holder are shown in Table 4.2.

\begin{tabular}{ccccc}
\hline Coil & Inner diameter: & Width: & Height: & Coil-sheet gap \\
& $77.8 \mathrm{~mm}$ & $3.17 \mathrm{~mm}$ & $6.35 \mathrm{~mm}$ & $0.5 \mathrm{~mm}$ \\
\hline Groove & Inner diameter: & Width: & Height: & Profile radius: \\
& $71.5 \mathrm{~mm}$ & $6.35 \mathrm{~mm}$ & $10.16 \mathrm{~mm}$ & $3.17 \mathrm{~mm}$ \\
\hline
\end{tabular}

Table 4.2 EM coil geometric parameters

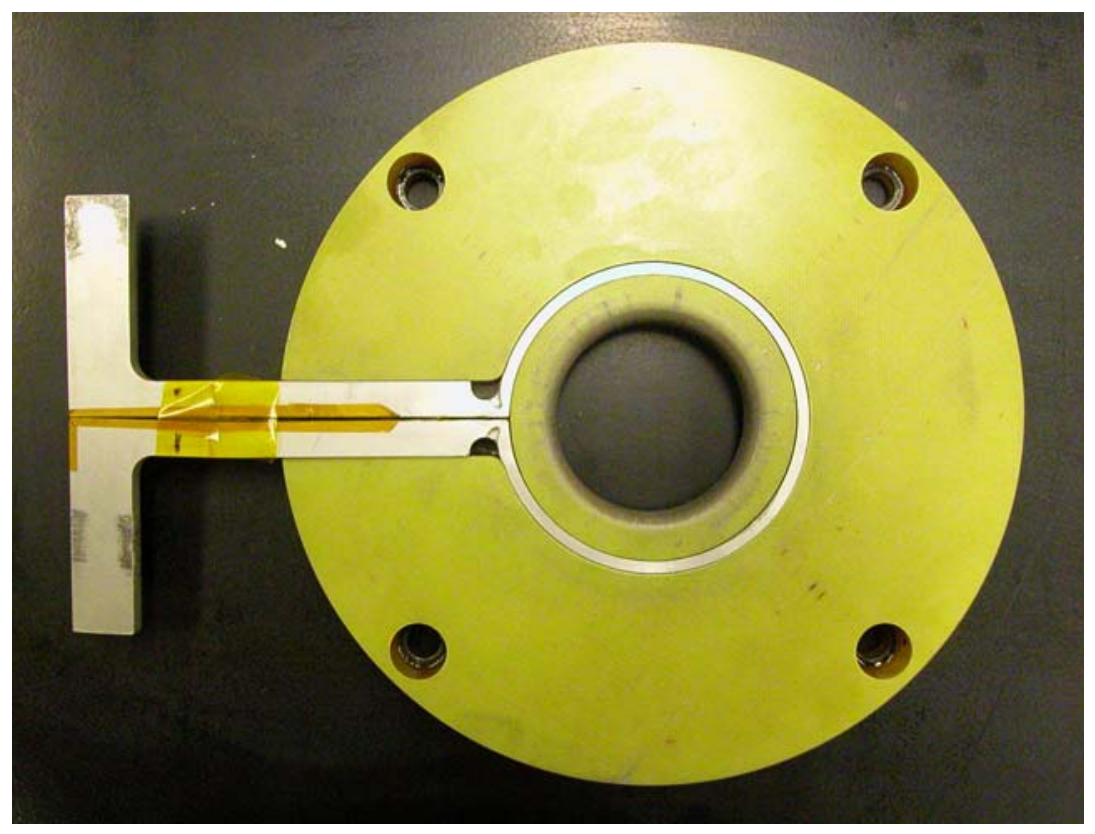

Figure 4.4 Electromagnetic coil embedded in the die of deep drawing 


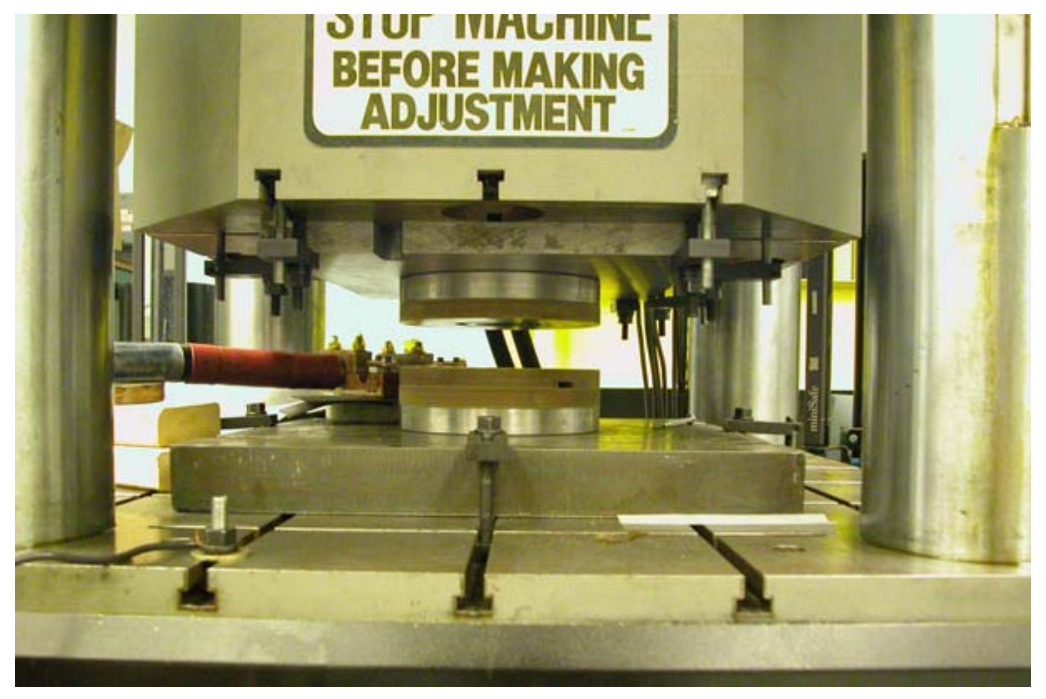

Figure 4.5 Photograph of the electromagnetically assisted deep drawing setup (the tooling and the coaxial coil)

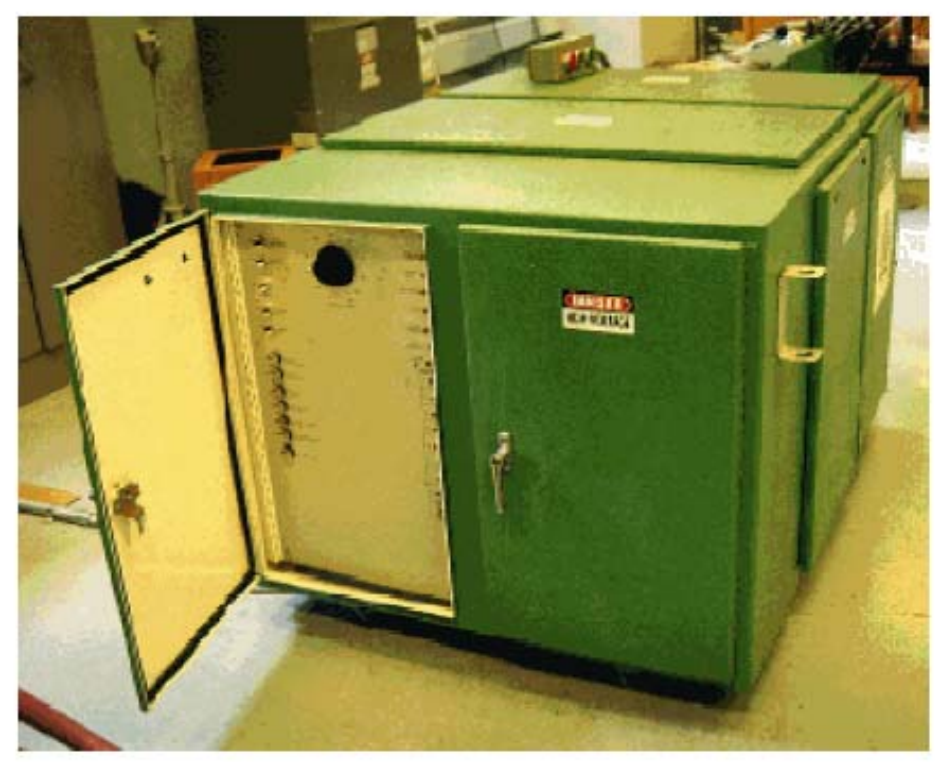

Figure 4.6 Photograph of the 48KJ capacitor bank 


\subsubsection{Material}

For the deep drawing experiment, aluminum alloy AA2219-O was used. The thickness is 8.13mm. The uniaxial tensile test [8] was carried out to measure the mechanical properties of this material in longitudinal, transverse and 45 degree directions. The results are shown in Table 4.3, and the stress-strain curves are shown in Figure 4.7.

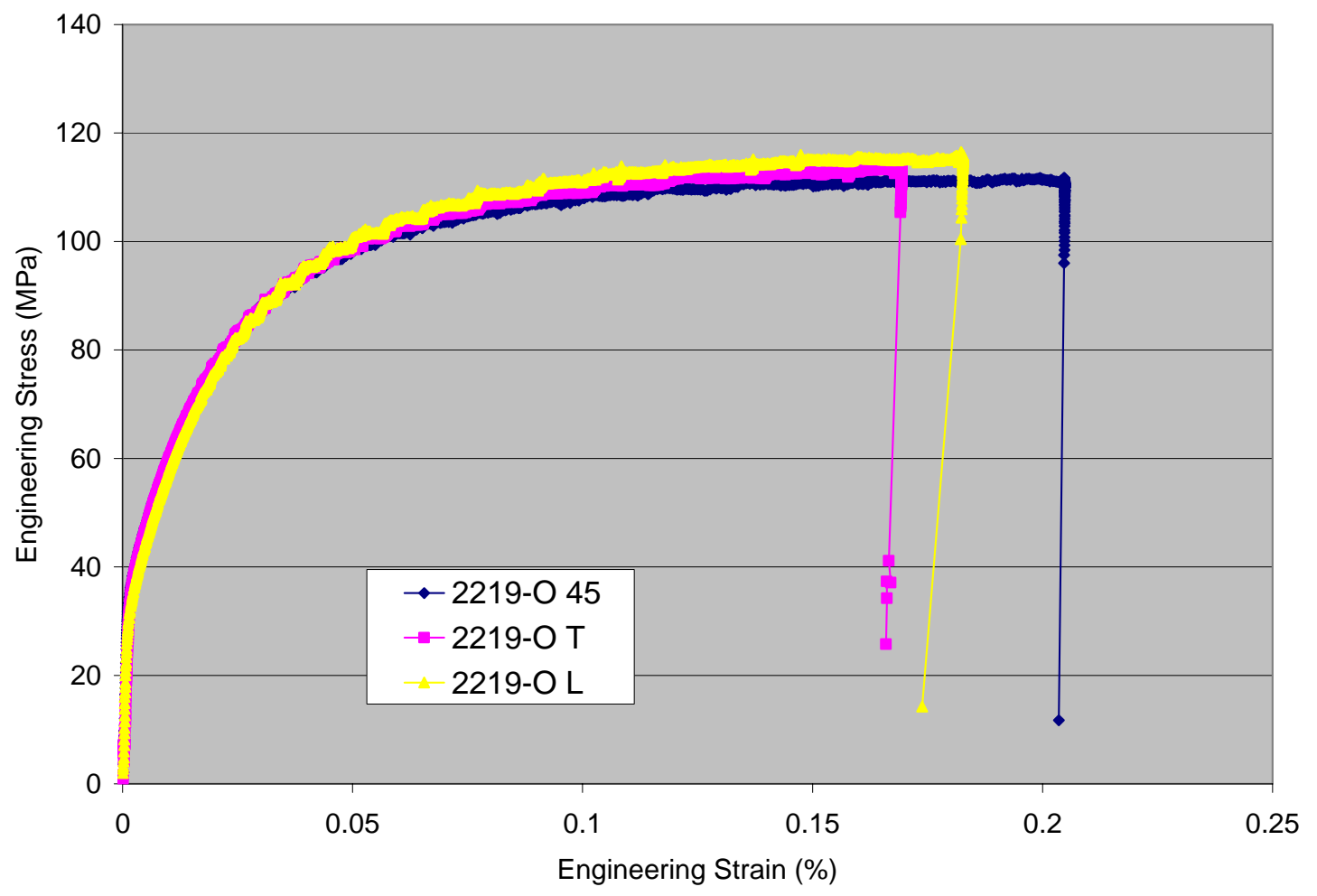

Figure 4.7 Engineering stress-strain plots for AA2219-O 


\begin{tabular}{cccccc}
\hline $\begin{array}{c}\text { Yield } \\
\text { strength } \\
(\mathrm{MPa})\end{array}$ & $\begin{array}{c}\text { Tensile } \\
\text { Strength } \\
(\mathrm{MPa})\end{array}$ & $\begin{array}{c}\text { Uniform } \\
\text { elongation } \\
(\%)\end{array}$ & $\begin{array}{c}\text { Total } \\
\text { Elongation } \\
(\%)\end{array}$ & $\begin{array}{c}\text { Strain } \\
\text { hardening } \\
\text { exponent }(\mathrm{n})\end{array}$ & $\begin{array}{c}\text { Plastic strain } \\
\text { ratio }(\bar{R})\end{array}$ \\
\hline 29.5 & 135 & 18 & 20 & 0.18 & 0.8 \\
\hline
\end{tabular}

Table 4.3 Mechanical tensile properties of AA2219-O sheet material

\subsubsection{Electromagnetic energy determination}

The electromagnetic coil is used to apply electromagnetic forces on the metal sheet to make the stretching. Electromagnetic forces are determined by the electromagnetic energy stored at the capacitor bank, for a given electromagnetic coil. So the electromagnetic energy level for pulses should be determined.

Several EM energy levels were tried to compare the effect on the "bubble" area. The experiment results showed that $4.2 \mathrm{~kJ}$ EM energy per pulse is suitable. It was determined that $4.2 \mathrm{~kJ}$ EM energy was applied for every pulse. Figure 4.8 is the photo of the initially flat metal sheet after one $4.2 \mathrm{~kJ}$ EM pulse. The line length of the "bubble" area was $14.9 \mathrm{~mm}$, and the extra line length caused by EM pulse was $2.2 \mathrm{~mm}$. So the engineering stretching strain was estimated as $17 \%$. This stretching strain was overestimated since the draw-in from the blank edge was not considered, as shown in Section 4.3.3.3. It should be notice that there was a lower section in the "bubble" ring right above the two leads of the EM coil. The reason is that there was not enough EM force at the area because of the 
gap between the two leads of the single turn EM coil. To make a "bubble" ring with uniform height, the metal blank was turned around 45 degree after every 3 EM pulses.

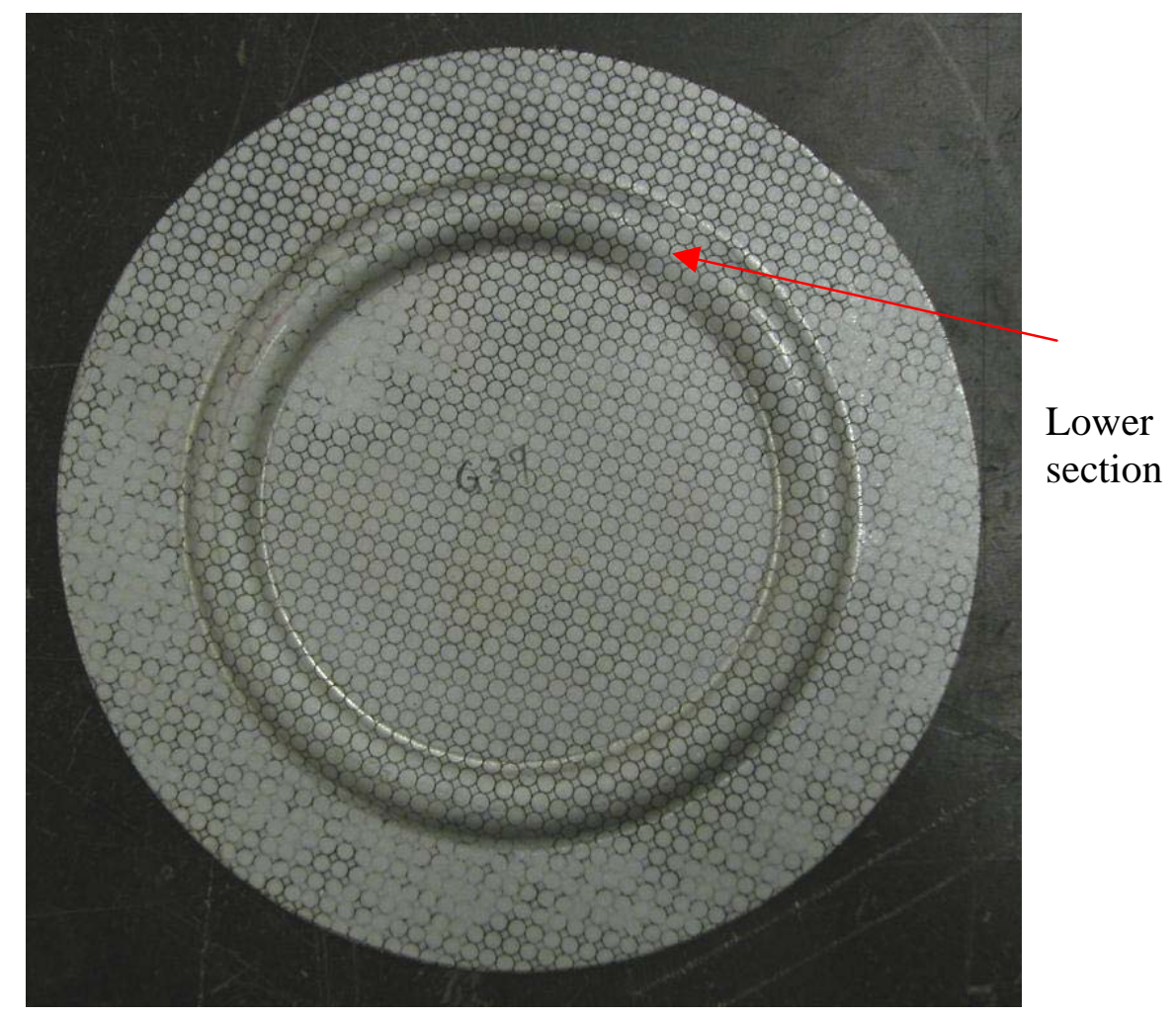

Figure 4.8 Photo of the initially flat metal sheet with $127 \mathrm{~mm}$ diameter after one $4.2 \mathrm{~kJ}$ EM pulse

Figure 4.9 is the current trace for the primary current in the EM coil for $4.2 \mathrm{~kJ}$ EM pulse. It shows that the peak current was $96 \mathrm{kA}$ and the rising time for the first peak current was $18 \mu \mathrm{s}$. 


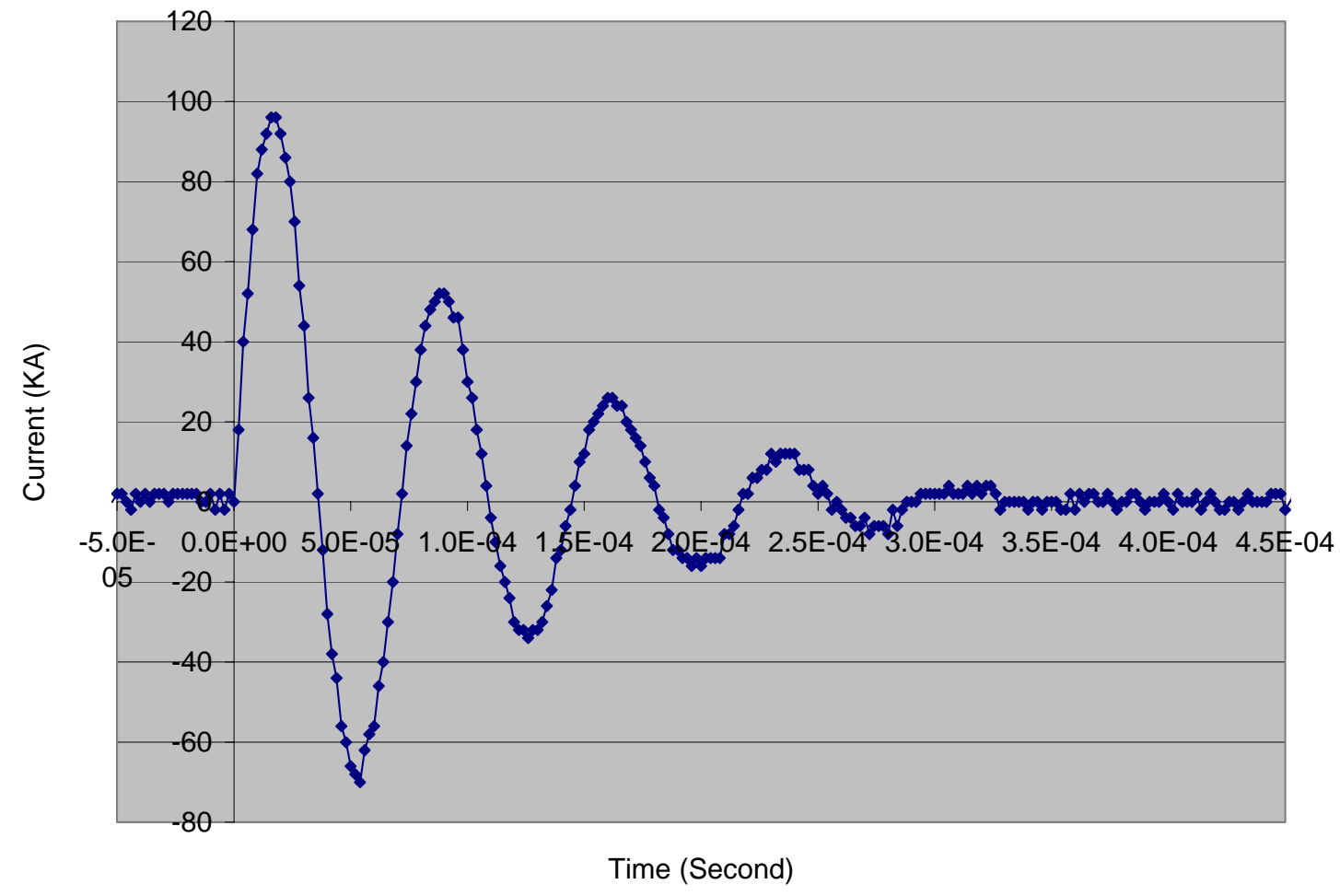

Figure 4.9 Primary current trace in the EM coil for 4.2 kJ EM pulse

To apply electromagnetic forming better, a 2D simulation about the primary and induced current for $4.2 \mathrm{~kJ}$ EM pulse was carried out with Maxwell-2D student version [86]. According to Figure 4.9, the primary current had the peak current of $96 \mathrm{kA}$ and the frequency of the primary current was $13.9 \mathrm{kHz}$. The electrical resistively of $\mathrm{Al} 2219-\mathrm{O}$ is $3.99 \times 10^{-6} \Omega-\mathrm{cm}$, and the one of $\mathrm{Al} 6061-\mathrm{T} 6$ is also $3.99 \times 10^{-6} \Omega-\mathrm{cm}$ [87]. Figure 4.10 shows the contour of the current density distributions of primary and induced currents. Figure 4.11 shows contour of the EM force density distribution in the metal sheet. Above two figures indicate that the EM force only has significant effect on the metal sheet very 
close to the EM coil. Above simulation was assumed that both EM coil and the metal sheet were stationary during the whole process, since Maxwell 2D can not model the metal deformation. But this is not the real case. Therefore, above simulation is only used to understand the basics of EM forming.

$J\left[\mathrm{~A} / \mathrm{m}^{\wedge} 2\right]$

$1.4384 \mathrm{e}+010$

$1.2946 \mathrm{e}+010$

$1.1507 e+010$

$1.0069 \mathrm{e}+010$

$8.6304 e+009$

7. $1920 \mathrm{e}+009$

$5.7536 \mathrm{e}+009$

$4.3152 \mathrm{e}+009$

$2.8768 \mathrm{e}+009$

$1.4384 e+009$

0.0000 e+000

Figure 4.10 Contour of the current density distributions of primary and induced currents 

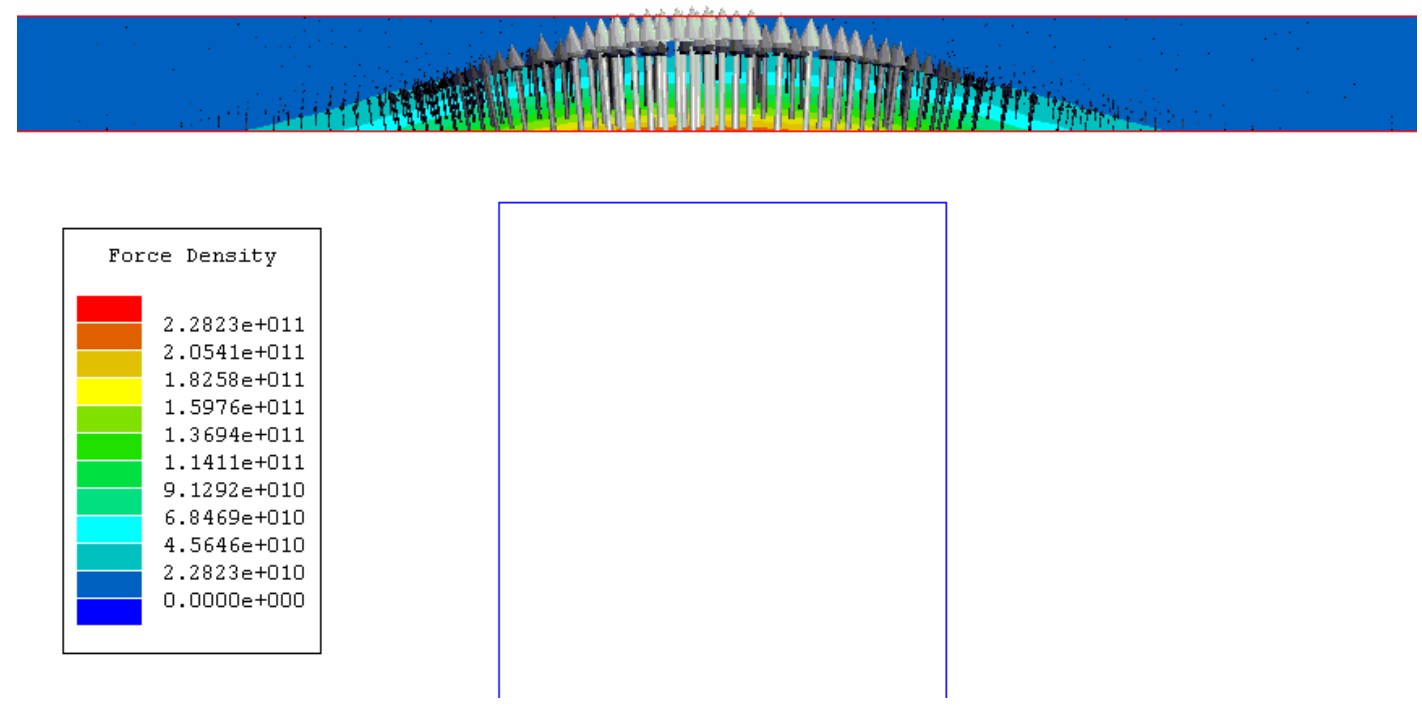

Figure 4.11 Contour of EM force density distribution in metal sheet

\subsubsection{Experiment procedures}

For conventional deep drawing, the experimental procedure was simple. The blank holder clamped the blank, and the punch advanced until the failure of the metal sheet.

For electromagnetically assisted deep drawing, the experiment procedure was relative complex, shown as below.

(1) The blank holder clamps the blank;

(2) The punch advances and stops at a pre-decided position;

(3) A electromagnetic pulse with predefined energy level is given to the coil and deforms the blank to make a stretched "bubble";

(4) Repeat (2) and (3) until the failure of the metal sheet; 
As mentioned in Section 4.15, the EM energy was set as $4.2 \mathrm{~kJ}$ for every EM pulse, which would produce about $2.2 \mathrm{~mm}$ extra line length. The punch advancement between each pulse should not be larger than that value to avoid the complete pulling out of the “bubble” ring. In this experiment, it was set as $1.25 \mathrm{~mm}$.

\subsection{Experiment results and discussions}

Table 4.4 are the experiment result summary. Detailed results will be introduced below and some discussion will also be presented.

\begin{tabular}{ccccccc}
\hline & $\begin{array}{c}\text { Blank } \\
\text { diameter } \\
(\mathrm{mm})\end{array}$ & $\begin{array}{c}\text { Clamp } \\
\text { load } \\
(\mathrm{N})\end{array}$ & $\begin{array}{c}\text { Energy } \\
\text { per pulse } \\
(\mathrm{KJ})\end{array}$ & $\begin{array}{c}\text { Pulse } \\
\text { time }\end{array}$ & $\begin{array}{c}\text { Cup } \\
\text { height } \\
(\mathrm{mm})\end{array}$ & Failure type \\
\hline Conventional \\
deep drawing & 95.3 & 2224 & 0 & 0 & 30.2 & No failure \\
\cline { 2 - 7 } & 101.6 & 2224 & 0 & 0 & 10.4 & Tearing at nose \\
\cline { 2 - 7 } & 127.0 & 1334 & 0 & 0 & 8.6 & Tearing at nose \\
\cline { 2 - 7 } & $254 \times 254$ & 4000 & 0 & 0 & 7.7 & Tearing at nose \\
\cline { 2 - 7 } Electromagne & $254 \times 254$ & 1334 & 0 & 0 & 5.4 & Wrinkling at flange \\
\cline { 2 - 7 } -tically & 101.6 & 2224 & 4.2 & 27 & 34.5 & Wrinkling at wall \\
\cline { 2 - 7 } drawing & 127.0 & 1334 & 4.2 & 25 & 30.9 & Tearing at nose \\
\cline { 2 - 7 } & 127.0 & 222 & 4.2 & 21 & 25.7 & Tearing at wall \\
\cline { 2 - 7 } & 127.0 & 2669 & 4.2 & 24 & 29.9 & Tearing at flange \\
\cline { 2 - 7 } & 127.0 & 1334 & 3.0 & 10 & 11.4 & Tearing at nose \\
\cline { 2 - 7 } & 127.0 & 1334 & 6.0 & 20 & 24.8 & Tearing at flange \\
\cline { 2 - 7 } & $254 \times 254$ & 4000 & 4.2 & 16 & 17.8 & Tearing at flange \\
\cline { 2 - 7 } & $254 \times 254$ & 1334 & 4.2 & 16 & 17.8 & Tearing at flange \\
\hline
\end{tabular}

* All experiments were applied lubrication. The lubricant is OAK Draw (777) \#6P.

Table 4.4 Experiment result summary 


\subsubsection{Measurement of LDR}

Axisymmetric deep drawing can be viewed as two processes: stretching sheet over a circular punch, and drawing an annulus flange inwards. The two operations are connected at the cylindrical cup wall, which transmits the force between both regions. The compression of the flange provides the major resistance force for drawing. The larger the flange is, the bigger the resistance is. If the cup wall can't provide enough strength to draw flange in, tearing will occur at the cup wall. And the blank holder should press the flange hard enough to prevent another type of failure, wrinkling. Therefore, for a given punch diameter and a given material, there must be a maximum permissible blank size for successful deep drawing, which leads to the maximum cup height.

The Limiting Drawing Ratio, LDR, is the measurement of the ratio of the maximum permissible blank size to the punch diameter [7]. To measure it for this experiment, several blanks with different diameters were carried out by conventional deep drawing at different clamp loads. The results showed that the biggest blank which can be completely drawn without tearing and wrinkling is $95.3 \mathrm{~mm}$ diameter. And the clamp load is $2224 \mathrm{~N}$. The draw depth is $30.2 \mathrm{~mm}$. So the value of LDR for AA2219-O can be calculated as below:

$$
L D R=\frac{95.3}{50}=1.91
$$




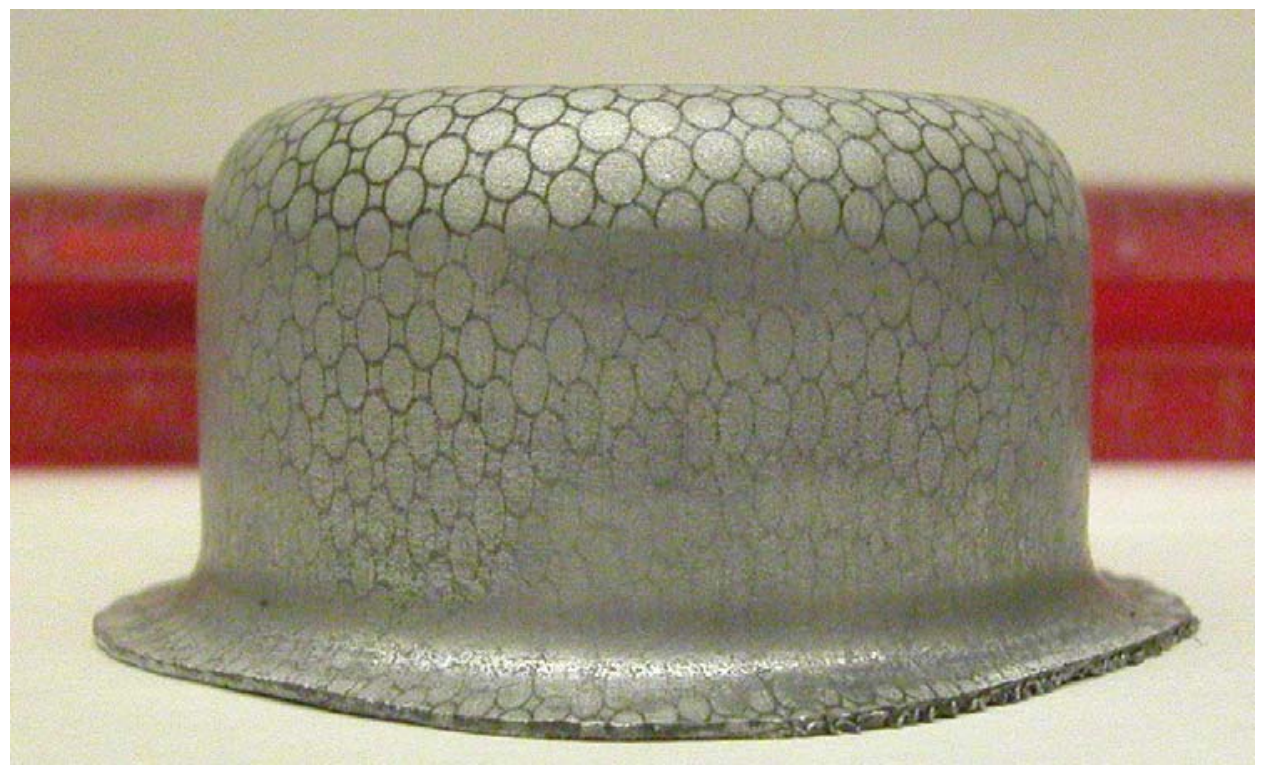

Figure 4.12 Photograph cup drawn from 95.3mm-diameter blank (2224N clamp load, 30.2mm cup height, and with lubrication; Material: AA2219-O)

\subsubsection{Blanks with $101.6 \mathrm{~mm}$ diameter}

Blanks with bigger diameter $(101.6 \mathrm{~mm})$ were used in both conventional deep drawing and electromagnetically assisted deep drawing. The results are shown in Figure 4.13. The left cup in Figure 4.13 was made by conventional deep drawing, and the left was made by electromagnetically assisted deep drawing. Because of the EM pulses, more material was drawn from the flange into die cavity, and the higher cup height was achieved. This is just the goal of the electromagnetic pulses. From this point, it is good to apply electromagnetic coil in the flange surface of the die. 


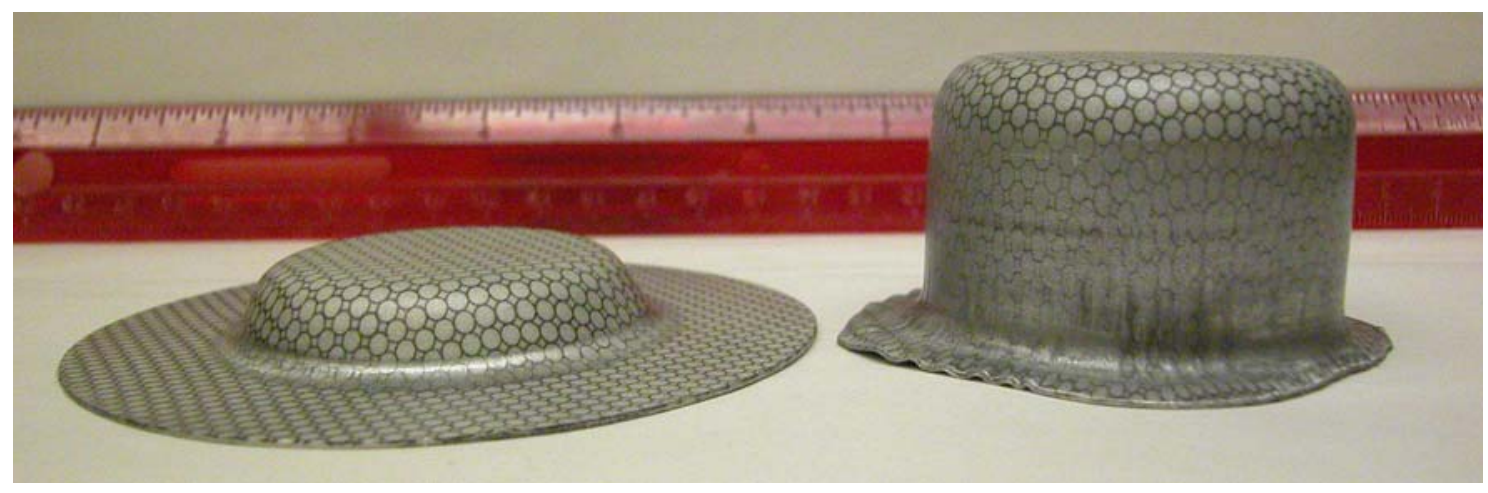

Figure 4.13 Photograph of cups drawn from 101.6mm diameter blank (Left: cup height 10.4mm with 2224N clamp load made by conventional deep drawing; Right: cup height $34.5 \mathrm{~mm}$ with $2224 \mathrm{~N}$ clamp load and 27 pulses)

With a larger blank size, the cup height made by EM pulses only increased by $14 \%$, comparing to the highest cup height made by conventional deep drawing, because there was serious wrinkling at the cup wall due to lacking of clamp forces above the coil.. Can this kind of failure be avoided? Checking the blank holder of the electromagnetically assisted deep drawing, there is hollow groove just above the coil. This is required because there must be space for the stretched "bubble" made by EM pulses. This hollow groove does benefit the draw-in of the flange. But it also makes it difficult to control wrinkling, epically when the rim of the flange passes. When the rim of the flange passes by the hollow groove, the rim actually becomes completely free of clamp load. At that time, wrinkling is inevitable at the rim of the flange. Therefore, a flat blank holder should be applied when the rim of flange passes by the coil to solve this problem, which requires the tooling change. 
While, if the rim of blank doesn't move into the hollow groove area, the two ends of the section of blank in the hollow groove are still being pressed against the die by the blank holder. It is still possible to prevent wrinkling by the blank holder. Therefore, EM assisted deep drawing is suitable to produce the cylindrical cup with some flange left. To prevent wrinkling at the hollow groove, one possible way is to decrease the width of the hollow groove, which will be presented in next discussion section.

According to above discussion, electromagnetic pulses can be suitable to form the parts with flange, or with compression which is not enough to lead to wrinkling. It is not suitable to form a part whose flange should be completely drawn into the die cavity.

\subsubsection{Blanks with $127.0 \mathrm{~mm}$ diameter}

\subsubsection{Drawing and stretching}

Larger blanks with $127.0 \mathrm{~mm}$ diameter were used to form part with flange. For conventional deep drawing, the cup will crack soon because of the large blank. But using EM pulses did dramatically improve the cup height. The results are shown in Figure 4.14. By electromagnetic pulses, the cup height increased by 3.6 times. 


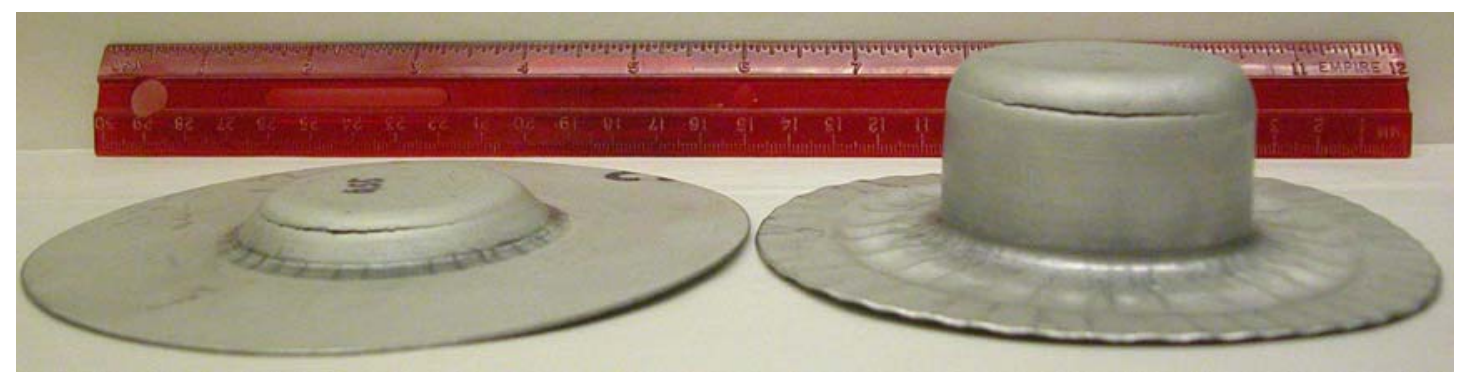

Figure 4.14 Photograph of cups drawn from 127.0mm diameter blank (Left: 8.6mm cup height with 1334N clamp load made by conventional deep drawing; Right: 30.9mm cup height with $1334 \mathrm{~N}$ clamp load and 25 electromagnetic pulses)

Comparison of the flange size after deep drawing shows that EM pulses did improve the draw-in of flange, as shown in Figure 4.15. Because of EM pulses, the diameter of flange was reduced about $14 \mathrm{~mm}$. As mentioned before, EM pulses were applied to stretch the flange area. So for this $127 \mathrm{~mm}$-diameter blank, EM pulse had two functions. One was to stretching the flange and the other one was to draw more metal into die cavity from the flange. The contributions of above two functions to the improvement of draw depth will be discussed below. 


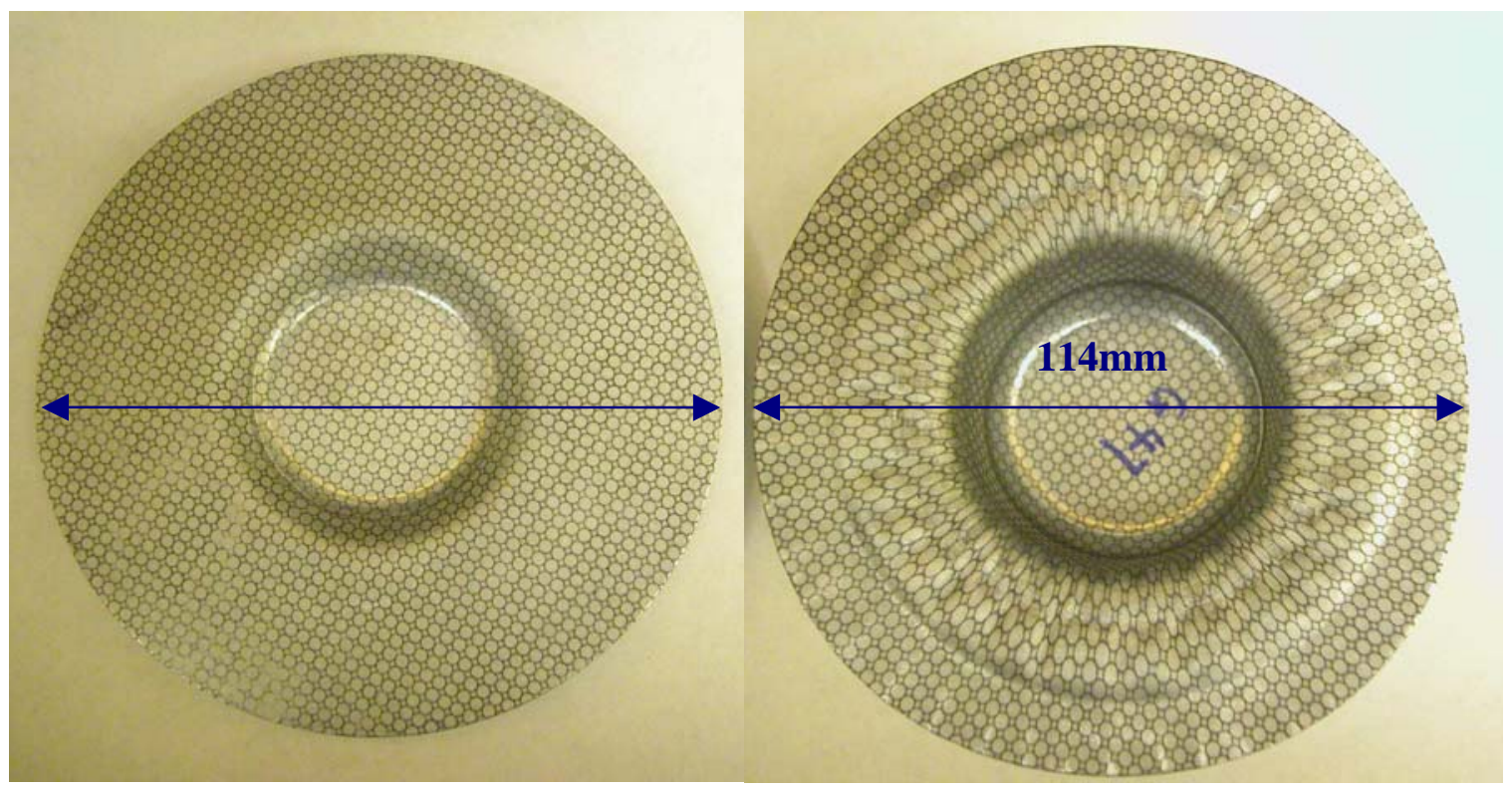

Figure 4.15 Comparison of the flange size after deep drawing, same samples in Figure 4.13 (Left: 126mm diameter; Right: 113mm diameter; Original: 127mm diameter)

If $14 \mathrm{~mm}$ metal annularity was drawn into die cavity from the flange, how much could the cup height be improved? Because of the volume conservation, if we suppose there was no thickness change and all the $14 \mathrm{~mm}$ metal annularity was converted to the side wall, an approximate estimate of the cup height improvement can be obtained as following:

$$
\frac{\pi d_{0}{ }^{2} t_{0}}{4}-\frac{\pi\left(d_{0}-\Delta d\right)^{2} t_{0}}{4}=\pi d_{p} t_{0} \Delta h
$$


Since initial blank diameter $d_{0}=127.0 \mathrm{~mm}$, punch diameter $d_{p}=50.0 \mathrm{~mm}$ and the diameter reduction $\Delta d=14 \mathrm{~mm}$, from Equation 4.2, the cup height improvement is given by

$$
\Delta h=\frac{d_{0}^{2}-\left(d_{0}-\Delta d\right)^{2}}{4 d_{p}}=16.8 \mathrm{~mm}
$$

The cup height increased about $22.3 \mathrm{~mm}$. About $16.8 \mathrm{~mm}$ improvement of cup height was from the improvement of draw-in from the flange, which means $75 \%$ of improvement of cup height came from the improvement of draw-in. Therefore, the improvement of drawin due to electromagnetic pulses is important. Equation 4.3 was assumed that all the diameter reduction was converted to the side wall. It was not true, since the metal was not flat at the area above the EM coil shown in Figure 4.9. So 75\% contribution of draw-in improvement to the cup height improvement was only the maximum value. The actual value must be less than that. But there should not be much error, since the area at the hollow groove was not wide and had small curve.

Above is about the effect of draw in. How about the stretching? Figure 4.15 clearly shows that there was almost no deformation at the flange in conventional forming, i.e. the radial strain and the hoop strain are about zero. Because of the EM pulses, the metals from the hollow groove to the die radius were dramatically stretched, which should be contributed to the improvement of draw depth. As shown in Figure 4.16, some area has very large stretching that even the radial strain reached $91 \%$. And the hoop strain is also increased to $-20 \%$. The area between the rim and the hollow groove had the different 
strain states. In this area, the radial and hoop strains were relative small. A simple estimate can be carried out to calculate the hoop strain of the edge of the flange, as shown following:

$e_{\text {edge }}=\frac{\pi\left(d_{0}-\Delta d\right)-\pi d_{0}}{\pi d_{0}}=\frac{-\Delta d}{d_{0}}=\frac{-14}{127}=-11 \%$

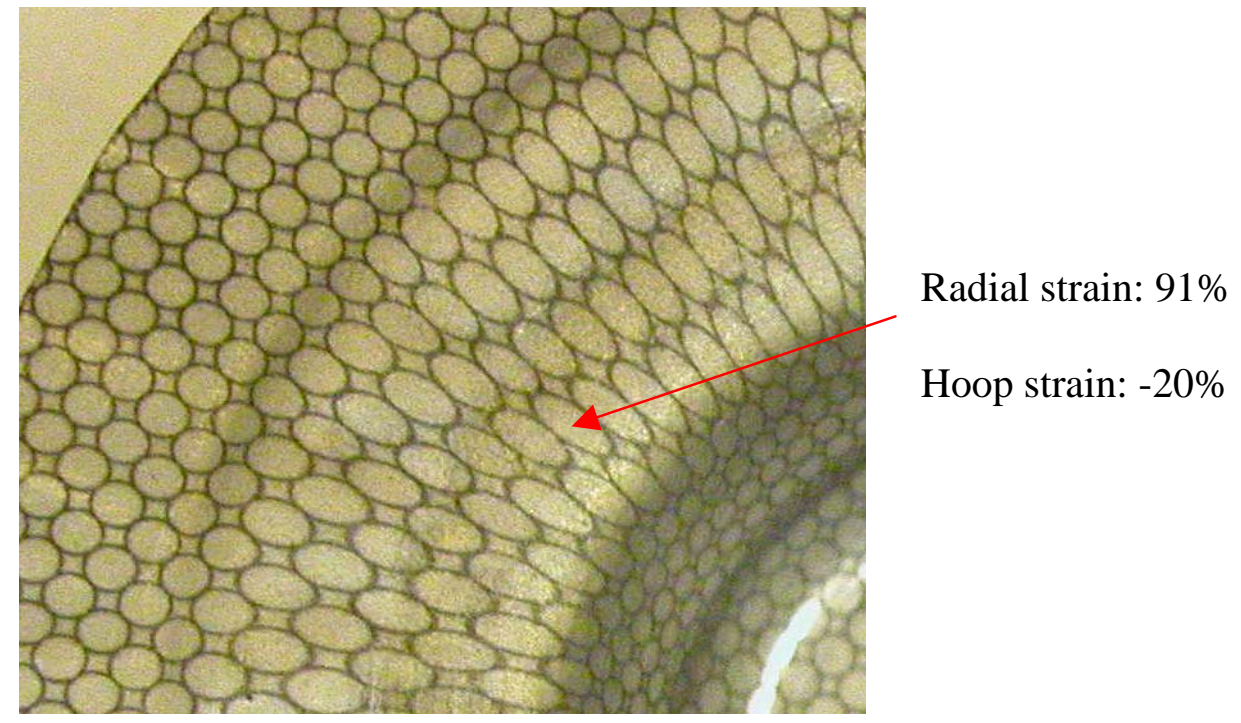

Figure 4.16 Large stretching on the flange caused by EM pulses (same sample shown in right of Figure 4.15)

The sample in the right of Figure 4.15 was made by electromagnetically assisted deep drawing with $127.0 \mathrm{~mm}$-diameter blank, which has the almost same cup height as the one made by conventional deep drawing with $95.3 \mathrm{~mm}$-diameter blank. And there is no wrinkling at the cup wall of the sample, although there is wrinkling at the flange of the sample. Comparison of the strain distributions of these two samples in Figure 4.17 shows 
that there is no difference at the bottom of parts. But there is large difference in the radial strain at the top of cup between these two samples. The difference comes from the stretching effect of the electromagnetic pulses.

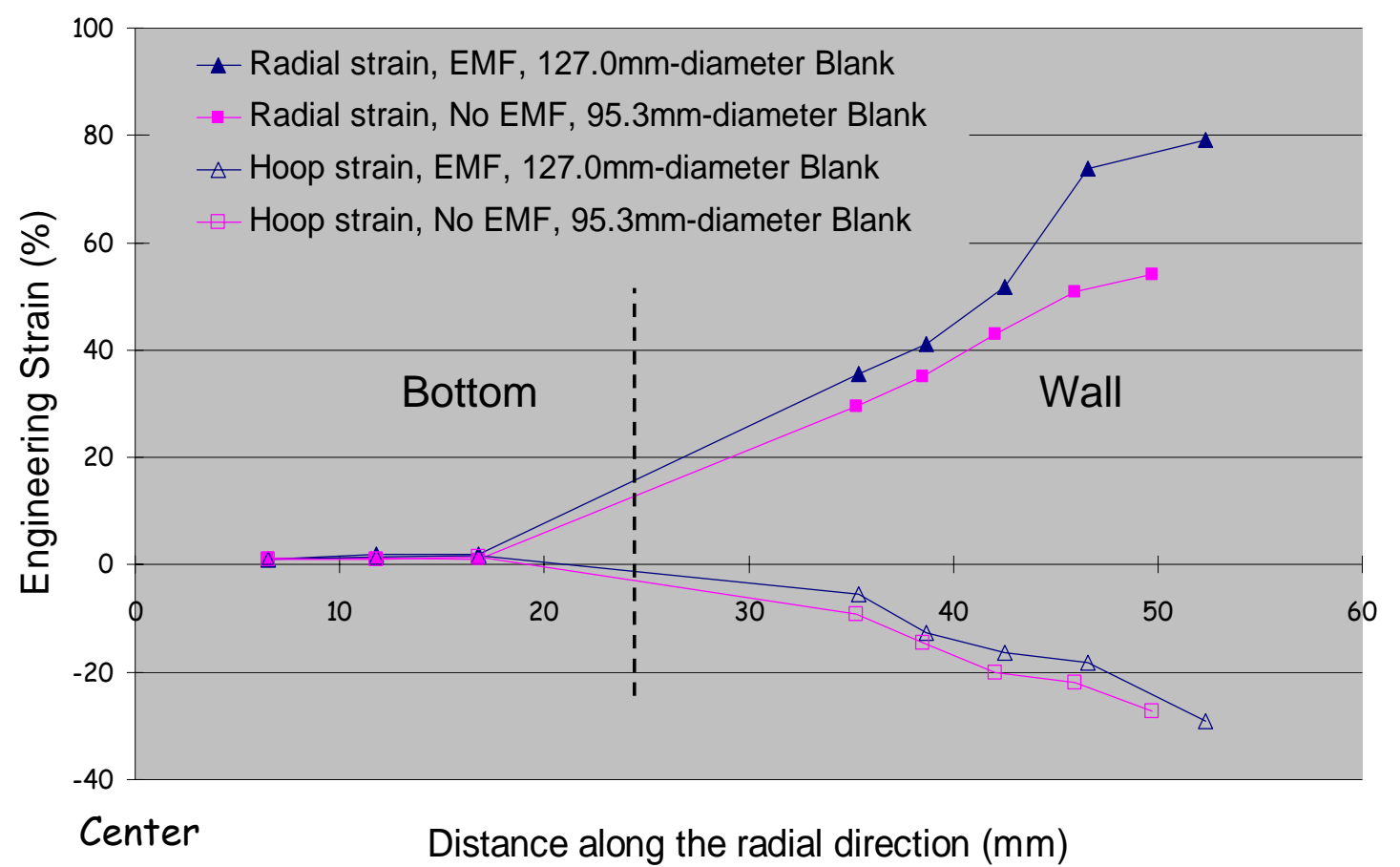

Figure 4.17 Comparison of strain distributions

From the comparison of the punch load shown in Figure 4.18, the punch load increases with the punch displacement and reaches the maximum value in the middle of the conventional deep drawing. For EM assisted case, the punch load increases with the punch displacement and keeps a relative constant value after some punch displacement. 


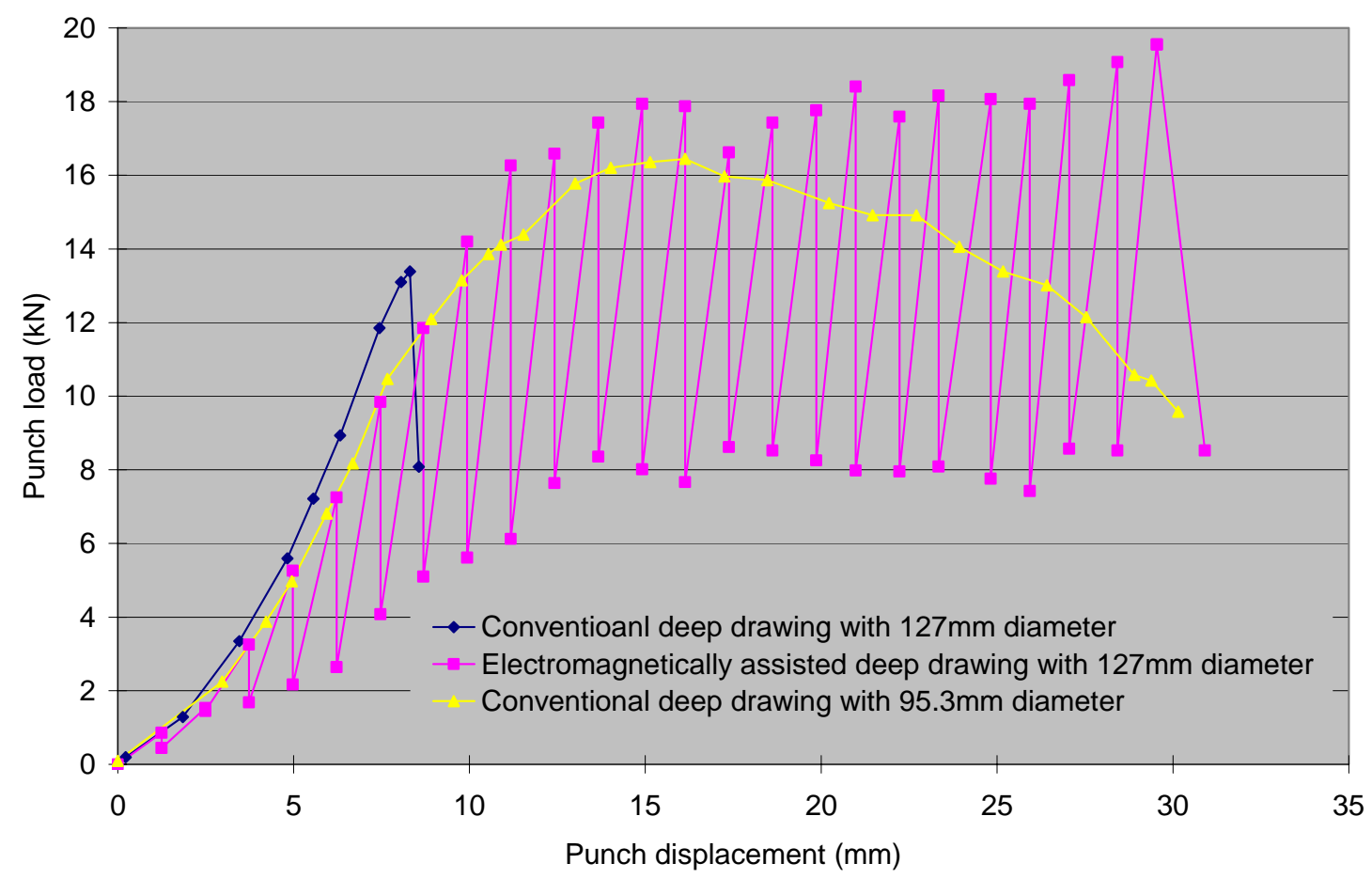

Figure 4.18 Comparison of strain distributions and the punch load

\subsubsection{Wrinkling and buckling}

The previous section shows a sample formed by electromagnetically assisted deep drawing. It is noticed that there is wrinkling at the flange, and there is serious buckling at the area above the electromagnetic coil. These wrinkling and buckling caused bad shape, and have to be addressed. 
For the wrinkling at the flange, as described before, it can be avoided by increasing clamp load. Experiment result shows that increasing clamp load to $2669 \mathrm{~N}$ prevented the wrinkling at the flange, shown in Figure 4.19. The flange size after the deep drawing is $117 \mathrm{~mm}$, which is bigger than the one made by electromagnetic pulses with $1334 \mathrm{~N}$. It's reasonable because the higher clamp load makes it difficult to draw metals in from the flange. Higher clamp load could eliminate wrinkling, but also increase the friction force to the draw-in. So clamp load should be carefully decided to be just big enough to prevent wrinkling.

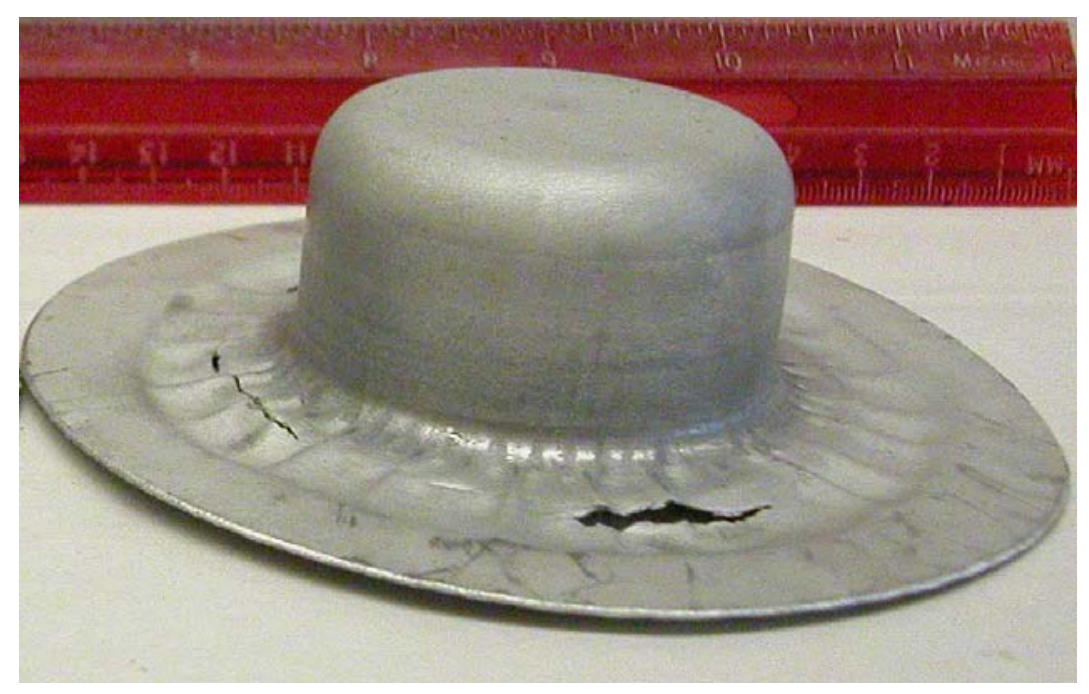

Figure 4.19 Photograph of cup drawn without wrinkling at flange (with original 127.0mm-diameter blank, 2669N clamp load and 24 EM pulses; after tearing at flange, cup height is $29.9 \mathrm{~mm}$ and flange diameter is $117 \mathrm{~mm}$ ) 
According to the Equation 4.2, the cup height improvement due to draw-in can be estimated as:

$$
\Delta h=\frac{d_{0}^{2}-\left(d_{0}-\Delta d\right)^{2}}{4 d_{p}}=\frac{127.0^{2}-117^{2}}{4 \times 50.0}=12.2 \mathrm{~mm}
$$

For this case, the cup height increased about $21.3 \mathrm{~mm}$. Then, 57\% of improvement of cup height came from the improvement of draw-in. Comparing to last sample, the contribution of draw-in decreased. It shows the effects of draw-in and stretching vary according to the different forming conditions. The less the draw-in is, the more important the stretching is.

While, there was still buckling above the EM coil in this sample. Since the buckling is relative to the compression in the hoop direction and the thickness of the sheet, less stretching will be helpful to avoid buckling, which certainly reduces the cup height. This is the price that must pay to avoid the buckling. How to design the coil and the hollow groove to prevent buckling will be discussed later in this chapter.

It's interesting to notice that EM pulse can eliminate the buckling. When buckling appeared, the sheets developed sinusoidal displacement above the EM coil. Then some areas were very close to the coil and others were far away from the coil. According to the estimate equation of electromagnetic pressure, $\frac{F}{l}=\frac{\mu_{0} I_{1} I_{2}}{2 \pi d}$, the electromagnetic pressure is related to the distance between the coil and workpiece. When the EM energy was delivered to the coil, the areas close to the coil experienced large electromagnetic pressure and were pushed out. While other areas away from the coil had small 
electromagnetic pressure and moved little. Therefore, after EM pulse, the buckling was reduced and the metal above the coil became smooth "bubble”. Meanwhile, more metal was drawn inwards from the flange and the metal above the coil was stretched again. When the punch continued to advance, metal was drawn from the "bubble" towards the die cavity. During this drawing, the metal above the coil buckled again, since the thickness became thinner and the compression became bigger. But more metal was still drawn into the die cavity.

By above method, more material can still be drawn into the die cavity to be part of cup wall, although the buckling may appear early. According to these observations, for the case in Figure 4.19, the metal above the coil became buckled when the cup height reached $13.9 \mathrm{~mm}$. The final cup height was $29.9 \mathrm{~mm}$. Although there was buckling, much more metal was still be drawn into the die cavity because EM pulses could reduce the effect of buckling.

\subsubsection{Effect of EM energy level}

EM energy delivered per electromagnetic pulse determines the magnitude of the stretching and the draw-in, because it defines the electromagnetic pressure for an electromagnetic forming system. The bigger electromagnetic pressure which is brought by higher electromagnetic energy level will make more stretching and meanwhile will draw more metals in from the edge of the flange. Different energy levels were applied in the experiments, as shown in Figure 4.20. The flange sizes of these cups were measured and are shown in Table 4.5. 

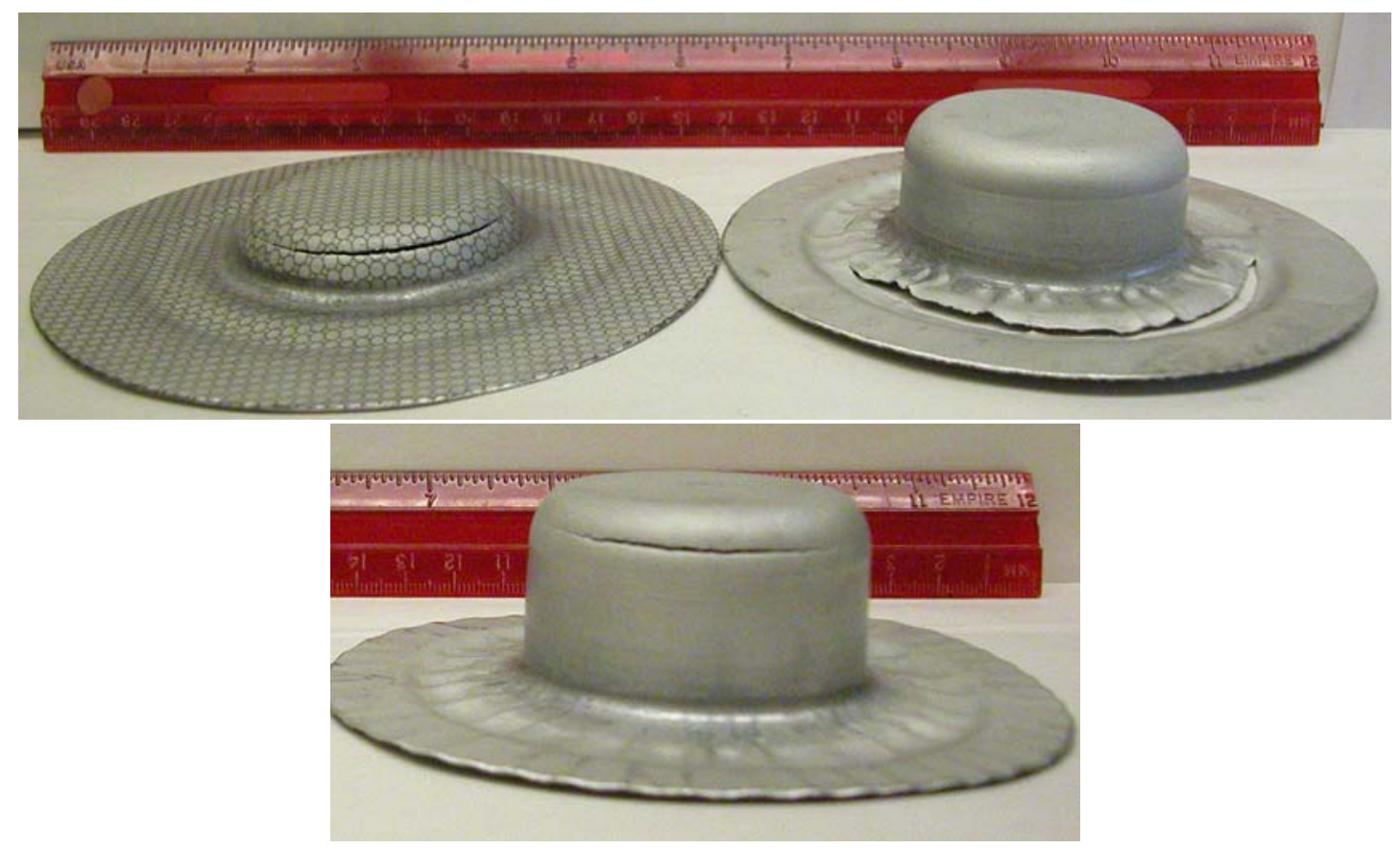

Figure 4.20 Photograph of cups drawn from 127.0mm diameter blank with different EM energy levels and same clamp load (Left: $11.4 \mathrm{~mm}$ cup height with 10 times of 3.0KJ EM pulses; Right: $24.8 \mathrm{~mm}$ cup height with 20 times of $6.0 \mathrm{KJ}$ EM pulses; Middle: $30.9 \mathrm{~mm}$ cup height with 25 times of 4.2 KJ EM pulses)

\begin{tabular}{cccc}
\hline & 3.0KJ, 10 times & 4.2KJ, 25 times & 6.0KJ, 20 times \\
\hline Flange size (mm) & 125 & 113 & 120 \\
Cup height improvement (mm) & 2.8 & 22.3 & 16.2 \\
$\begin{array}{c}\text { Cup height improvement due to } \\
\text { draw-in (mm) }\end{array}$ & 1.3 & 15.5 & 7.4 \\
\hline $\begin{array}{c}\text { Contribution to cup height duo } \\
\text { to draw-in }\end{array}$ & $46 \%$ & $70 \%$ & $46 \%$ \\
\hline
\end{tabular}

Table 4.5 Flange sizes and cup height of samples with different EM energy level 
For this case, 3.0KJ per pulse did not provide enough electromagnetic pressure to stretch the metals and meanwhile to draw the metals in. Therefore, the sample cracked soon. While 6.0KJ per pulse gave too much pressure to metals. So the metals were stretched too much and became too thin, which could not provide enough force to draw the edge of the flange in and crack soon.

Previous analysis shows the importance of the EM energy level. Low energy can't stretch and draw enough metals. While high energy stretches too much and weakens the metal. Suitable energy level should be carefully determined according to different conditions.

\subsubsection{Blanks with $177.8 \mathrm{~mm}$ diameter}

In previous experiments, blank sizes were not large enough to effectively eliminate the draw in. So EM pulses could stretch the metals and meanwhile draw metal inwards from the flange outside of the hollow groove. Now the question is if this method is good for very large or even infinite blank. In this section, very large blanks with $177.8 \mathrm{~mm}$

diameter were used to form cylindrical cup by conventional deep drawing and electromagnetic assisted deep drawing. The results are shown in Figure 4.21. 


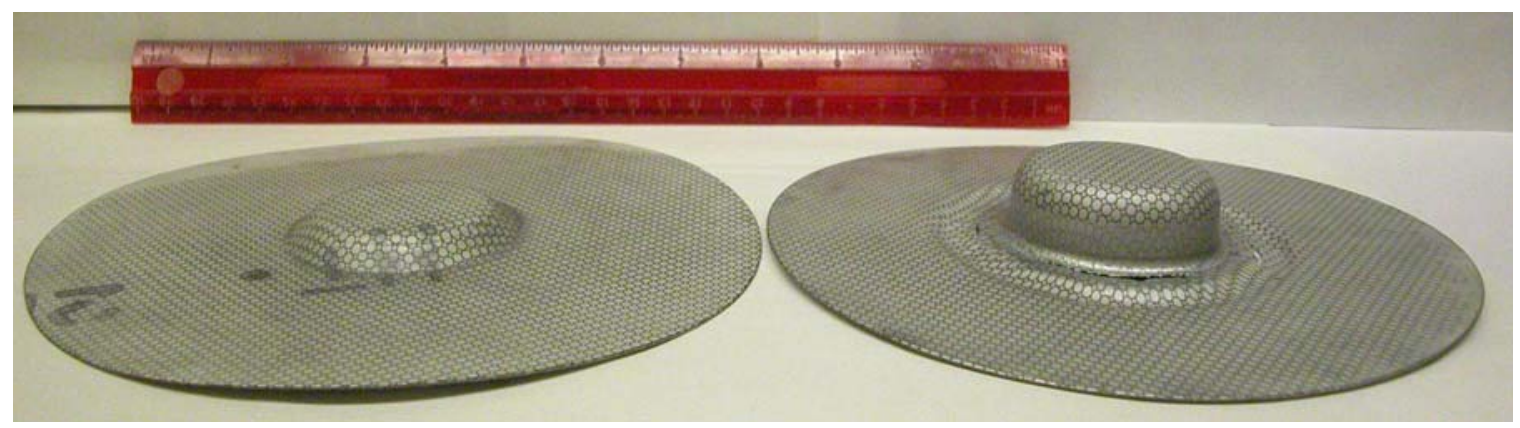

Figure 4.21 Photographs of cups drawn from blanks with $177.8 \mathrm{~mm}$ diameter (Left: 7.7mm cup height with 2669N clamp load made by conventional deep drawing; Right: 19.1mm cup height with 2669N clamp load made by 18 times of EM pulses)

The results indicate that the cup height was improved by 2.5 times. Did the cup height improvement come completely from the stretching? The measurement shows that the flange diameter of right cup is $175.3 \mathrm{~mm}$ and the one of left cup is $177.8 \mathrm{~mm}$. According to the equation 4.2 and assuming all the draw-in were converted to side wall, the cup height improvement due to draw-in can be estimated as following:

$$
\Delta h=\frac{d_{0}^{2}-\left(d_{0}-\Delta d\right)^{2}}{4 d_{p}}=\frac{177.8^{2}-175.3^{2}}{4 \times 50.0}=4.4 \mathrm{~mm}
$$

And the cup height improvement is $11.4 \mathrm{~mm}$. So $39 \%$ of the cup height improvement comes from the draw-in due to the electromagnetic pulses (maximum value since the flange was not complete flat). Although the blanks were very large, there was a little bit draw-in from the flange outside of hollow groove caused by electromagnetic pulses. The draw-in still had contribution to the improvement of cup height. 


\subsubsection{Blanks with $254 \mathrm{~mm}$ square}

To investigate the effect of stretching with maximum restrain from draw-in, $254 \mathrm{~mm} \times$ $254 \mathrm{~mm}$ square blanks were used to form the parts by conventional and electromagnetically assisted deep drawing.

\subsubsection{Drawing with high clamp load}

To prevent wrinkling of the edge, higher clamp load was applied in both conventional and EM assisted deep drawing. The results are shown in Figure 4.22.

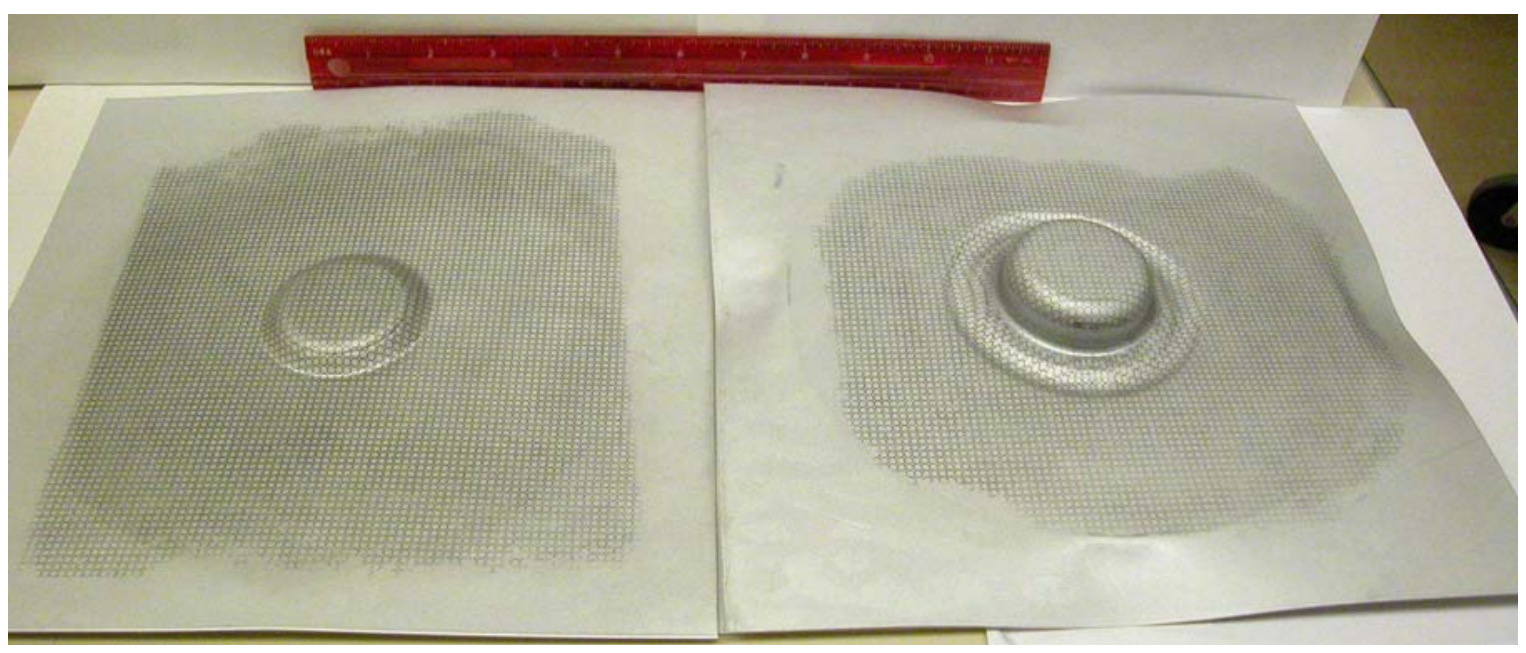

Figure 4.22 Photograph of cups drawn from $254 \mathrm{~mm} \times 254 \mathrm{~mm}$ square blanks (Left: 7.7mm cup height with 4000N clamp load made by conventional deep drawing; Right: 17.8mm cup height with 4000N clamp load made by 16 times of EM pulses) 
To completely block the draw-in from the flange, the flange was bent in electromagnetically assisted deep drawing. And the measurement showed that there was no draw-in from the flange. Therefore, for this case, the cup height improvement was improved by 2.3 times and all came from the stretching of metals inside of the hollow groove. Comparing to the previous cases with smaller blank diameters, the cup height improvement is the smallest. It's reasonable because there was no draw-in from the flange outside of the hollow groove.

It is interesting to compare the strain distribution of above two samples. The biggest difference between them is the strain at the flange area inside the hollow groove. Figure 4.23 are the photograph of the flange of the cups. The whole flange of the cup had little deformation. The hoop strain and radial strain are very small and close to zero. But for the case of electromagnetically assisted deep drawing, the flange had two distinct areas. The first one is from the rim of the flange to the hollow groove, which has almost no deformation same as conventional deep drawing. The second one is from the hollow groove to the die radius, which has very small hoop strain and very large radial strain. Some area has 53\% radial strain and about 0 hoop strain, as shown in Figure 4.23. 


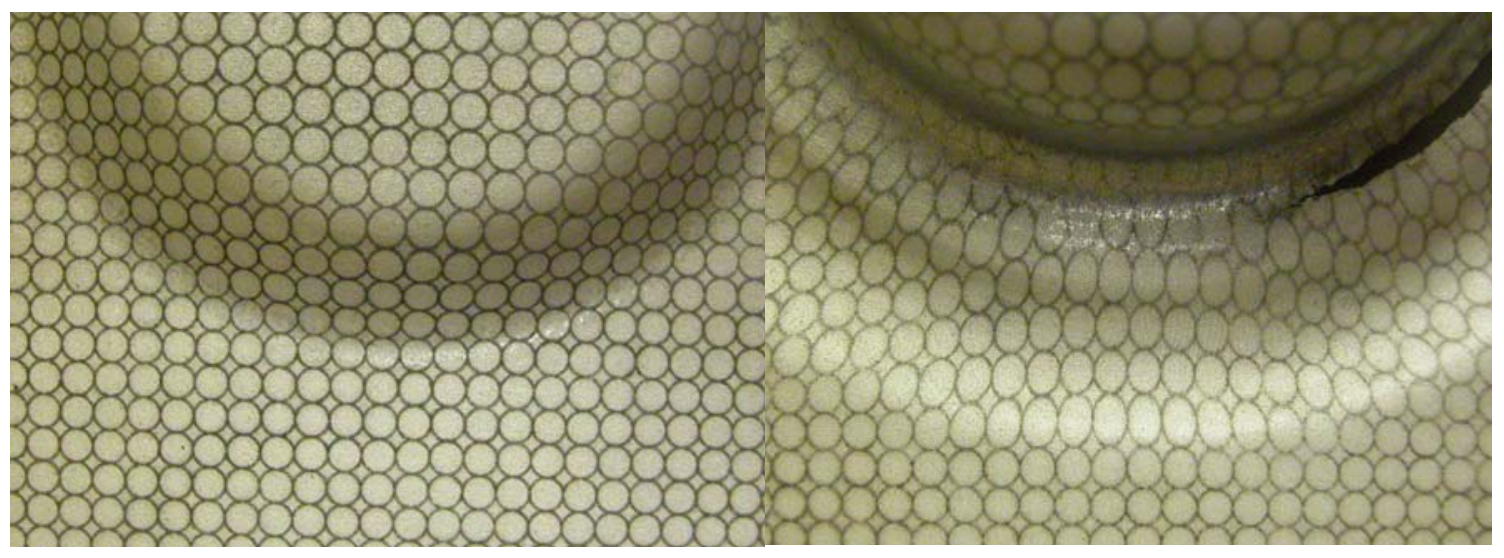

Figure 4.23 Photograph of the flanges of cups (Left: made by conventional deep drawing same as the left in Figure 4.22; Right: made by electromagnetically assisted deep drawing same as the right in Figure 4.22)

It is reasonable. Because the blank size is so large, there is no draw-in from the flange of the cup caused by the electromagnetic pulses. Then the electromagnetic pulses have little effect on the first area. For the second area, since no metal can be drawn into the hollow groove, the only extra metals flowing into the die cavity are caused by the stretching due to electromagnetic pulses. The metals above the coil are stretched again and again and have large radial strain. Meanwhile, the hoop strain of this area is small because no metal flows into the hollow groove. Therefore, there is no buckling in this case, although the deformation is large.

As described before, some areas have a nearly plane strain state and the major strain reaches 53\%. It is much larger than the forming limit of plane strain state in FLD, which 
is generally close to $20 \%$ for aluminum alloys. It must be relative to the different stress states and strain rates encountered in the electromagnetic forming. In electromagnetic forming of these experiments, the forming time was less than $30 \mu$ s. Although delivered electromagnetic energy is small, the electromagnetic forces still causes high velocity forming, which makes it possible to improve the forming limits of the metal.

\subsubsection{Drawing with low clamp load}

The previous section was carried out with high clamp load to prevent wrinkling at the flange. The blanks were also drawn with low clamp load. The experiment results are shown in Figure 4.24.

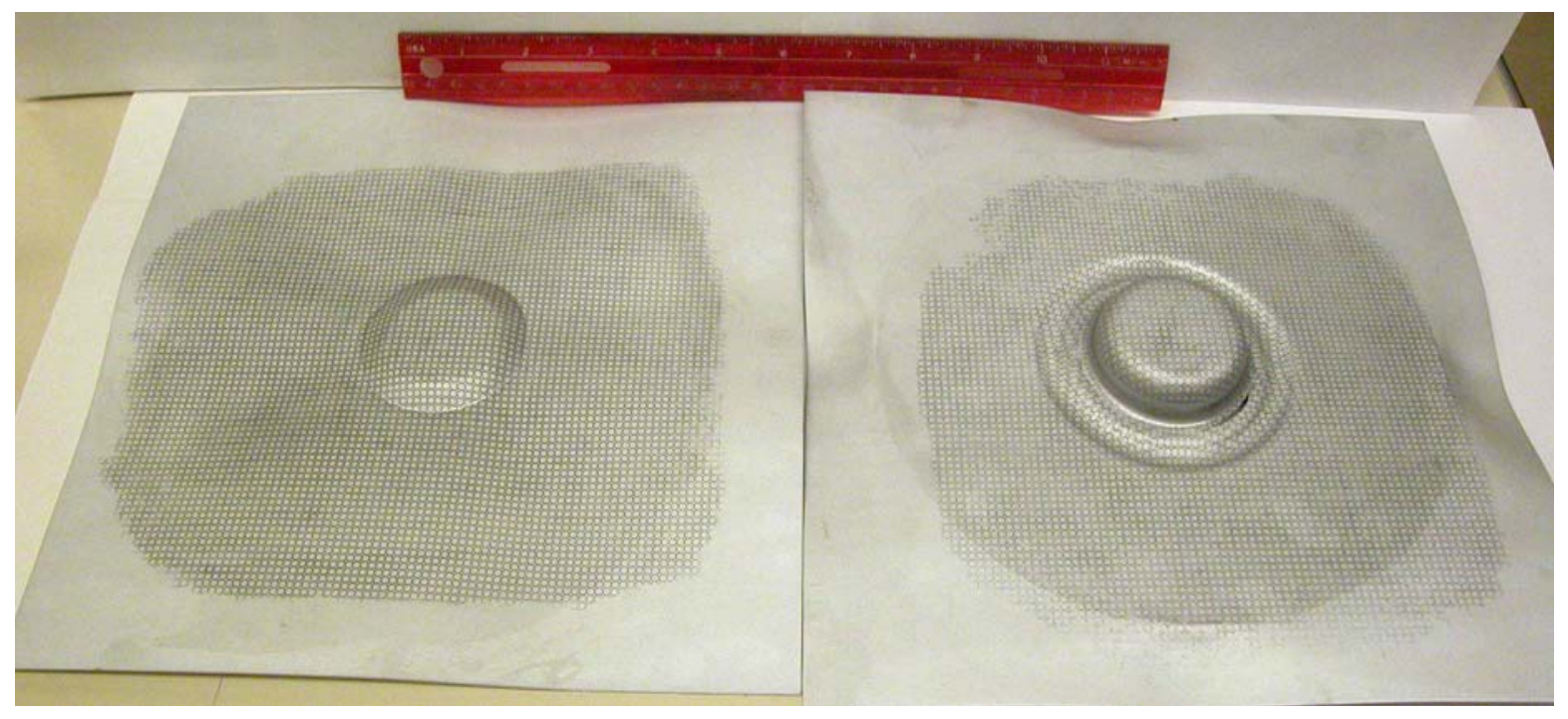

Figure 4.24 Photograph of the cups drawn $254 \mathrm{~mm} \times 254 \mathrm{~mm}$ square blanks (Left: $5.4 \mathrm{~mm}$ cup height with 1334N clamp load made by conventional deep drawing; Right: 17.8mm cup height with $1334 \mathrm{~N}$ clamp load made by 16 times of EM pulses) 
When the clamp load was low, wrinkling happened at the flange area in conventional deep drawing. When the punch advanced, the metals were drawn a little from the flange near die radius to the die cavity. Although the draw-in was small, there was still large tension force at the flange, which in turn led to the large compressive stress states. There was wrinkling when the clamp load was not large enough to press down the flange.

For the EM case, the cup height was the same as the one with high clamp load. It must be due to the effect of EM pulses. The EM pulses stretched the metals above the coil, which made a new boundary condition for the blank. The deformation from the hollow groove to the die cavity had little effect on the metals outside of the hollow groove. So no matter high or low clamp load, there was no wrinkling and the cup heights were same.

\subsubsection{Summary}

Previous sections are the detailed experiment results and some discussion. There are several important facts in electromagnetically assisted deep drawing:

(1) Multiple electromagnetic pulses with small energy do improve the draw-in from the flange and do stretch the flange area above the electromagnetic coil, which contribute the improvement of the cup height in deep drawing;

(2) There are three types of failures: tearing, wrinkling and buckling. Tearing may happen at the area near the punch nose, or at the area inside of the hollow groove. Wrinkling often happens at the edge of the flange. And buckling usually occurs at the area inside of the hollow groove; 
(3) The wrinkling can be avoided by the increased clamp load. But for the buckling, there is little method to avoid except the reduction of deformation, because there is no clamp load in the hollow groove above the coil;

(4) The contributions to the cup height of draw-in and stretching are different according to the blank size. The smaller the blank size, the smaller the contribution of the stretching. For the very large or infinite blank size, the stretching is the only source for the cup height improvement;

(5) Small electromagnetic energy is applied for every electromagnetic pulse. The forming cause by small EM energy may still be high velocity forming and cause the improvement of the forming limit.

To further understand the mechanism of electromagnetically assisted deep drawing, theoretical analysis for these two forming process will be presented below.

\subsection{Theoretical Analysis}

Below 2D analytical models for conventional and electromagnetically assisted deep drawing will be presented. In the models, materials is assumed to be rigid-plastic and the strain hardening characteristics is of the form [7],

$$
\bar{\sigma}=K(\bar{\varepsilon})^{n}
$$

where $\mathrm{K}$ is the material constant, $\bar{\sigma}$ is the flow stress, $\bar{\varepsilon}$ is the flow strain and $n$ is the strain hardening exponent. The material properties are assumed to be isotropic in the sheet plane so that there is only a normal anisotropy $\bar{R}$. 
Material is assumed to be yielding according to Hill's anisotropic criterion and expressions for flow stress $\bar{\sigma}$ and increments strain $d \bar{\varepsilon}$ [88] are given (neglecting shear components) by

$$
\begin{aligned}
& \bar{\sigma}=\sqrt{\frac{1}{1+\bar{R}}}\left[\left(\sigma_{\theta}-\sigma_{z}\right)^{2}+\left(\sigma_{r}-\sigma_{z}\right)^{2}+\bar{R}\left(\sigma_{r}-\sigma_{\theta}\right)^{2}\right]^{1 / 2} \\
& d \bar{\varepsilon}=\frac{\sqrt{1+\bar{R}}}{1+2 \bar{R}}\left[\left(d \varepsilon_{\theta}-\bar{R} d \varepsilon_{z}\right)^{2}+\left(d \varepsilon_{r}-\bar{R} d \varepsilon_{z}\right)^{2}+\bar{R}\left(d \varepsilon_{r}-d \varepsilon_{\theta}\right)^{2}\right]^{1 / 2}
\end{aligned}
$$

And then the flow rule [88] is given by

$$
\frac{d \varepsilon_{r}}{\bar{R}\left(\sigma_{r}-\sigma_{\theta}\right)+\left(\sigma_{r}-\sigma_{z}\right)}=\frac{d \varepsilon_{\theta}}{\bar{R}\left(\sigma_{\theta}-\sigma_{r}\right)+\left(\sigma_{\theta}-\sigma_{z}\right)}=\frac{d \varepsilon_{z}}{\left(\sigma_{z}-\sigma_{\theta}\right)+\left(\sigma_{z}-\sigma_{r}\right)}=\frac{d \bar{\varepsilon}}{(1+\bar{R}) \bar{\sigma}}
$$

Here $\sigma_{r}, \sigma_{\theta}$ and $\sigma_{z}$ are stresses and $d \varepsilon_{r}, d \varepsilon_{\theta}$ and $d \varepsilon_{z}$ are strain increments in $r, \theta$ and z directions respectively.

Generally the electromagnetic pulses finish the deformation in less than $100 \mu$ s, which leads to large strain rate. The constitutive equation below should be applied [88]

$$
\bar{\sigma}=K \bar{\varepsilon}^{n}(\dot{\varepsilon})^{m}
$$

While, for aluminum alloys in room temperature, $m$ generally is small [7]. For 6111-T4, $m$ is negative [111]; for 2219, $m$ is less than 0.04 [80, 89]. And the introduction of $m$ will make the model complex. Actually, the development of constitutive equation for plastic flow in high velocity forming is still in active research [53-56]. So for the 
simplified 2D analytical analysis, the effect of strain rate sensitivity will not be considered in this chapter.

\subsubsection{Theoretical analysis of conventional deep drawing}

For conventional axisymmetric deep drawing, there are clearly three important regions: the bottom which is stretched over a circular punch, the flange which is drawn inwards, and the wall which connects and transmits the force between above two regions [88].

In the flange area, the metal is drawn by tension in the radial direction and compressed in the circumferential direction to permit it to flow over the die lip. At the outer edge of the flange, there is a free surface and the stress state is therefore one of uniaxial compression. The blank will thicken as it deforms. While, at some intermediate radius, the radial stress will be equal and opposite of the hoop stress. And there is no change in thickness. At the inner edge near the die opening radius, the radial stress will be a maximum. And the sheet will thin. The metals of the flange will continually be drawn into the die cavity and become part of the cup wall [88].

The metals at the bottom area below the punch will experience the bi-axial stretching. And the sheet becomes thinner. This area generally has small deformation because of the friction between the punch and the sheet. 
As described before, the cup transmits the tension force to draw metal from the flange into the die cavity [90]. When the wall deforms, the punch will prevent circumferential straining, so the wall must deform in plane strain state. While, if the flange is too large, the force that must be transmitted by the wall will exceed the load-carrying capacity of the wall, therefore causing the wall to fail [90].

Classic theoretical analyses for conventional deep drawing can be found in many places in the literature [90-101]. Basically, there are four components of the resistance forces for deep drawing [91]:

(1) Plastic deformation due to the flange shrink;

(2) Plastic deformation due to the bending and unbending when metals flow above the die arc;

(3) Friction at the flange rim;

(4) Friction at the die arc;

If the resistance force exceeds the maximum force that the cup wall can provide, then the instability which will be leading to failure will occur. For a successful deep drawing, the key point is to make sure the cup wall have enough strength to draw the flange into the die cavity [92].

The deep drawing analysis is based on the work of Leu [92], who estimated the largest possible initial blank diameter that can be successfully drawn from a given cup diameter. Figure 4.25 [91] shows the schematic diagram for a cup-drawing operation in which a 
circular blank of original radius $R_{0}$ and thickness $t_{0}$ is deep drawn by a punch having radius $r_{c 0}$ through a die opening of radius $r_{c d}\left(r_{c d}=r_{c 0}+r_{d}\right)$. And $r_{d}$ is the edge radius of the die.

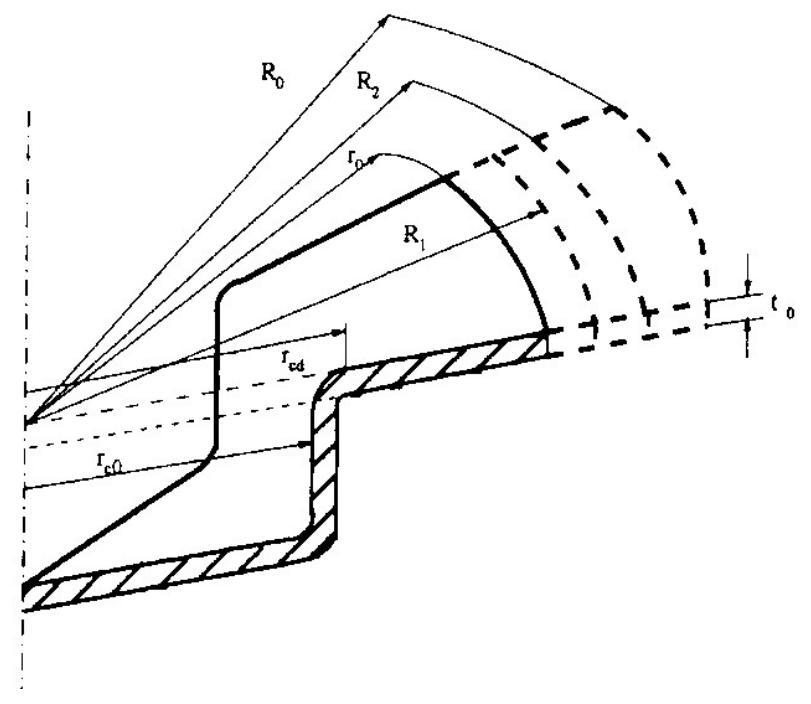

Figure 4.25 Schematic representation of a partial drawn cup [91]

As described before, there are four components for the resistance force of the deep drawing, which will be discussed individually below.

\subsubsection{Plastic deformation due to flange shrink}

The stresses acting on an element in the flange region at the current radius $r$ are shown in Figure 4.26 [91]. Radial equilibrium of the element gives 


$$
\frac{d \sigma_{r}}{d r}=-\frac{\left(\sigma_{r}-\sigma_{\theta}\right)}{r}
$$

Under plane stress condition ( $\sigma_{z}=0$ ), the relationship between of stresses $\sigma_{r}$ and $\sigma_{\theta}$ can be obtained from Eq. (4.7) as

$$
\sigma_{r}-\sigma_{\theta}=\bar{\sigma}\left[\frac{2(1+\bar{R})}{2 \bar{R}+1}\right]^{1 / 2}
$$

And the effective strain $\bar{\varepsilon}$ in the flange region is evaluated from plane strain deformation $\left(d \varepsilon_{z}=0\right)$ and incompressibility conditions from Eq. (4.8) as

$$
\bar{\varepsilon}=\sqrt{\frac{2(1+\bar{R})}{2 \bar{R}+1}}\left(-\varepsilon_{\theta}\right)
$$

where the hoop strain in the flange region is $-\varepsilon_{\theta}=\ln (R / r) . R$ is the initial radius of the annular ring which reaches radius $r$ after the cup has been partially drawn. From Eq. (4.6) and (4.13), effective stress in the flange can be obtained as

$$
\bar{\sigma}=K\left[\sqrt{\frac{2(1+\bar{R})}{2 \bar{R}+1}} \ln \left(\frac{R}{r}\right)\right]^{n}
$$

Using Eqs. (4.11), (4.12) and (4.14) and integrating from $r_{c d}$ to $r_{0}$ for the initial radius $R_{0}$ of the blank, the radial drawing stress at radius $r_{c d}$ in the flange can be obtained as [91]

$$
\int_{r_{c d}}^{r_{0}} d \sigma_{r}=-K\left[\sqrt{\frac{2(1+\bar{R}}{2 \bar{R}+1}}\right]^{1+n} \int_{r_{c d}}^{r_{0}}\left(\ln \left(\frac{R}{r}\right)\right)^{n} \frac{d r}{r}
$$


The above equation can be written as

$$
\sigma_{r_{c d}}=K\left[\sqrt{\frac{2(1+\bar{R})}{2 \bar{R}+1}}\right]^{1+n} \int_{r_{c d}}^{r_{0}}\left(\ln \left(\frac{R}{r}\right)\right)^{n} \frac{d r}{r}
$$

Eq. (4.16) can be used to calculate the radial stress caused by the plastic deformation due to the flange shrink.

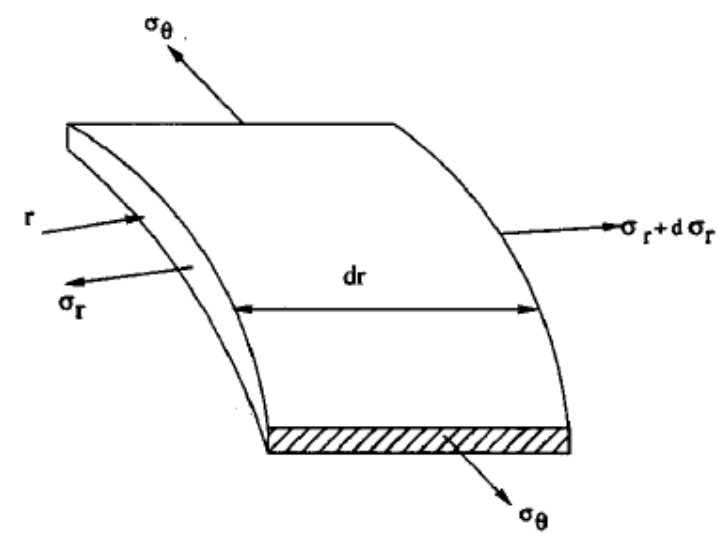

Figure 4.26 Radial stresses in the flange element [91]

\subsubsection{Friction on the flange rim}

Friction between the blank holder and the flange will also increase the drawing stress. It is a reasonable approximation to assume that the clamp load $B$ will be distributed around the edge of the flange as a line force of magnitude $B / 2 \pi r_{0}$ per unit length, since the outer edge of the flange gets thicken [88]. The friction force on the flange, per unit length 
around the edge, is thus $2 \mu \mathrm{B} / 2 \pi r_{0}$. This can be expressed as stress acting on the edge of the flange [88], i.e.

$$
\sigma_{f}=\frac{\mu B}{\pi r_{0} t}
$$

where $\mu$ is friction coefficient, $B$ is the clamp load, $t$ is the blank thickness, and $r_{0}$ is the current radius of the blank edge.

\subsubsection{Friction at the die arc}

Except the friction on the flange rim, there is another friction at the die arc region as the sheet metals slides along the die corner into the die cavity during deep drawing between radii $r_{c 0}$ and $r_{c d}$. At the die arc region, the stresses at $r_{c 0}$ and $r_{c d}$ can be given by $\sigma_{r_{c 0}}=\sigma_{r_{c d}} \exp \mu \frac{\pi}{2}$

where $\sigma_{r_{c d}}$ is the radial drawing stress at radius $r_{c d}$ in radial drawing of the flange region, $\sigma_{r_{c 0}}$ is the radial stress at radius $r_{c 0}$, and $\mu$ is friction coefficient.

\subsubsection{Plastic deformation due to bending and unbending}

When metals flow over the die arc, the blank will experience bending and unbending. And this leads the additional resistance force for drawing. The increased radial drawing stress due to the bending and unbending can be estimated as [12]

$$
\sigma_{w}=\frac{\sigma_{b} t}{2 r_{d}+t}
$$


where $\sigma_{b}$ is the tensile strength of the sheet metal, $r_{d}$ is the die arc radius and $t$ is the metal thickness.

The four components of the resistance force are presented above in Eqs. (4.16), (4.17) (4.18) and (4.19). Therefore, the stress which needs to transmit in the cup wall to draw the flange into die cavity can be written as

$\sigma_{r_{c 0}}=\left(\sigma_{r_{c d}}+\sigma_{f}\right) \exp \left(\mu \frac{\pi}{2}\right)+\sigma_{w}$

i.e. $\quad \sigma_{r_{c 0}}=\left[K\left[\sqrt{\frac{2(1+\bar{R})}{2 \bar{R}+1}}\right]^{1+n} \int_{r_{c d}}^{r_{0}}\left(\ln \left(\frac{R}{r}\right)\right)^{n} \frac{d r}{r}+\frac{\mu B}{\pi r_{0} t}\right] \exp \left(\mu \frac{\pi}{2}\right)+\frac{\sigma_{b} t}{2 r_{d}+t}$

Above equation can be expressed as a function of punch displacement or cup height as follows:

$\sigma_{r_{c 0}}=\left[K\left[\sqrt{\frac{2(1+\bar{R})}{2 \bar{R}+1}}\right]^{1+n} \int_{r_{c d}}^{\sqrt{R_{0}^{2}-2 r_{c 0} h}}\left(\ln \left(\sqrt{1+\frac{2 r_{c 0} h}{r^{2}}}\right)\right)^{n} \frac{d r}{r}+\frac{\mu B}{\pi r_{0} t}\right] \exp \left(\mu \frac{\pi}{2}\right)+\frac{\sigma_{b} t}{2 r_{d}+t}$

where $\mathrm{h}$ is the punch displacement or cup height, $R_{0}$ is the initial blank radius.

And the drawing force can be written as

$F=2 \pi r_{c 0} t_{0}\left[\left[K\left[\sqrt{\frac{2(1+\bar{R})}{2 \bar{R}+1}}\right]^{1+n} \int_{r_{c d}}^{\sqrt{R_{0}^{2}-2 r_{c 0} h}}\left(\ln \left(\sqrt{1+\frac{2 r_{c 0} h}{r^{2}}}\right)\right)^{n} \frac{d r}{r}+\frac{\mu B}{\pi r_{0} t}\right] \exp \left(\mu \frac{\pi}{2}\right)+\frac{\sigma_{b} t}{2 r_{d}+t}\right]$ 


\subsubsection{Critical condition in cup wall}

In deep drawing, load gets transmitted through the cup wall. If the drawing force exceeds a critical value, the instability which will be leading to failure will occur. Critical drawing force $\left(F_{c}\right)$ is the maximum force which the cup wall can provided. Generally the instability happens at the beginning of the punch nose radius in the cup wall $[7,88]$. This area is in the plane strain state, $d \varepsilon_{\theta}=0$, since the wall circumference is constrained by the punch from shrinking. And it is also in the plane stress state, $\sigma_{z}=0$. According to classic deep drawing analysis [7, 88], the critical radial strain is given as, $\varepsilon_{r}=n$. The critical effective strain and the critical radial stress in the cup wall can be expressed as

$$
\begin{aligned}
& \bar{\varepsilon}=\frac{1+\bar{R}}{\sqrt{1+2 \bar{R}}} n \\
& \sigma_{r c}=K\left(\frac{1+\bar{R}}{\sqrt{1+2 \bar{R}}}\right)^{1+n}(n)^{n}
\end{aligned}
$$

And the critical drawing force in the cup wall can be evaluated as [92]

$$
F_{c}=2 \pi r_{c 0} t_{0} K\left(\frac{1+\bar{R}}{\sqrt{1+2 \bar{R}}}\right)^{1+n}\left(\frac{n}{e}\right)^{n}
$$

The radial drawing stress $\sigma_{r_{c 0}}$ given in Eq. (4.20) should be always lower than the critical radial stress $\sigma_{r c}$ given in Eq. (4.24) to make a successful deep drawing. So the drawing force $\mathrm{F}$ in Equation 4.22 should be always smaller than the critical drawing force $\mathrm{F}_{\mathrm{c}}$. Otherwise, the tearing will happen at the side wall near punch nose. Therefore, the initial 
blank radius $\mathrm{R}_{0}$ has the limit value since the larger blank radius will cause larger resistance force due to the flange shrink, shown as in Equation 4.22.

\subsubsection{Theoretical analysis of electromagnetically assisted deep drawing}

The biggest difference between the conventional deep drawing and electromagnetically assisted deep drawing is the stretched "bubble" at the flange made by electromagnetic pulses, as shown in Figure 4.27. This stretched "bubble" has three functions. One is to stretch the metals. The second one is to draw metals inwards the hollow groove. The last function is to reduce the resistance force caused by flange shrink. These three functions will be discussed in detail below.

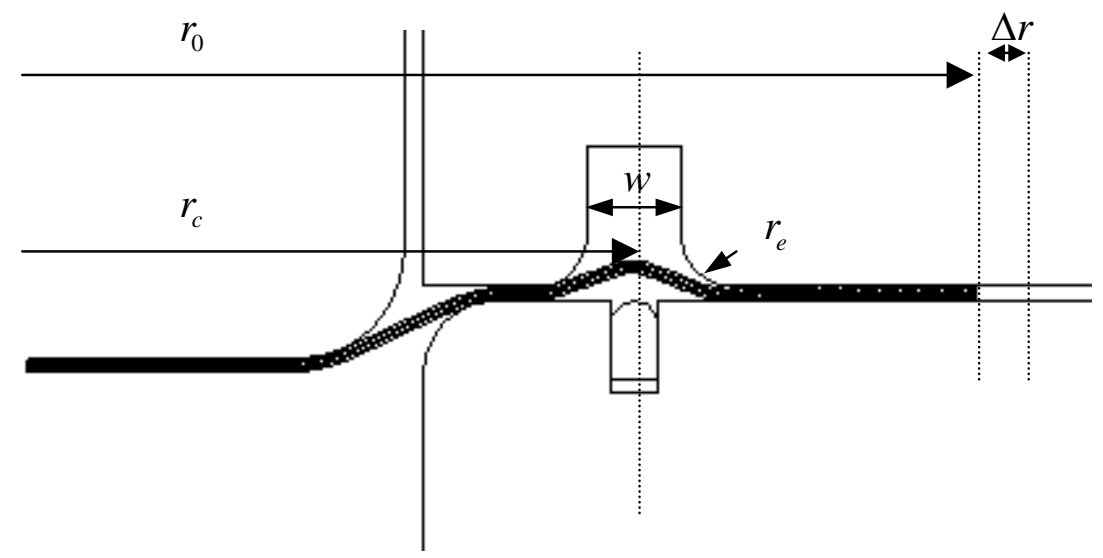

Figure 4.27 Schematic part drawn cup of electromagnetically assisted deep drawing ( $r_{0}$ is the current radius of blank, $r_{c}$ is the radius of coil center, $r_{e}$ is the edge radius of the hollow groove in blank holder, $w$ is the width of coil and $\Delta r$ is the draw-in caused by electromagnetic pulse) 


\subsubsection{Stretching and draw-in}

Every electromagnetic pulse will stretch the metals inside of the hollow groove and make a curved shape similar as "bubble". Meanwhile, more metals will be drawn into the hollow groove from the outer flange. According to the delivered energy level and the current blank diameter, the amounts of stretching and drawing are different. And the extra line length provided by stretching and drawing will be drawn inwards the die cavity and becomes part of cup wall during the movement of punch. After the curved "bubble" is pulled out, another electromagnet pulse will be given and a new "bubble" will be formed.

To simplify the analysis, above two events that happen simultaneously will be considered sequentially. First, the draw-in will be discussed. Assuming the blank radius is reduced by $\Delta r$, and there is no thickness change in the flange region, so according to the volume constancy, the metals to draw into the hollow groove can be estimated as

$$
\begin{aligned}
& \pi\left(R_{0}\right)^{2}-\pi\left(R_{0}-\Delta r\right)^{2}=2 \pi r_{c} \Delta l \\
& \text { i.e. } \quad \Delta l=\frac{\Delta r\left(2 R_{0}-\Delta r\right)}{2 r_{c}}
\end{aligned}
$$

where $r_{0}$ is the current radius of the blank edge, $r_{c}$ is the radius of the coil center.

Therefore, the metals in the hollow groove are considered to have a radial line length $\left(w+2 r_{e}+\Delta l\right)$ instead of $\left(w+2 r_{e}\right)$. It should be noticed that the metals only can be drawn into the hollow groove from the outer edge of the flange during above draw-in event. So during the draw-in event, at the inner side of the hollow groove, the metal has 
zero draw-in and then has zero circumferential strain. While, at the outer side of the hollow groove, the metal has the maximum draw-in and then has the maximum hoop strain. According to the volume constancy and assuming there is no thickness change in the flange region, the hoop strain at the outer side of the hollow groove can be estimated as

$\pi\left(R_{0}\right)^{2}-\pi R^{2}=\pi r_{0}^{2}-\pi\left(r_{c}+r_{e}+w / 2\right)^{2}$

i.e. $R=\sqrt{\Delta r\left(2 R_{0}-\Delta r\right)+\left(r_{c}+r_{e}+w / 2\right)^{2}}$

then $\varepsilon_{\theta-\text { out }}=\ln \frac{r_{c}+r_{e}+w / 2}{R}=\ln \frac{r_{c}+r_{e}+w / 2}{\sqrt{\Delta r\left(2 R_{0}-\Delta r\right)+\left(r_{c}+r_{e}+w / 2\right)^{2}}}$

It is reasonable to assume that the metals inside of the hollow groove should have different hoop strains which are linear function to their positions. For a position with relative line distance $\Delta L_{p}$ to the inner side of the hollow groove $\left(r_{c}-r_{e}-w / 2\right)$, i.e. the current radius is $r_{f}$, its hoop strain can be given as

$\varepsilon_{\theta}=\frac{\Delta L_{P}}{w+2 r_{e}+\Delta l} \varepsilon_{\theta-\text { Out }}=\frac{\Delta L_{P}}{w+2 r_{e}+\Delta l} \ln \frac{r_{c}+r_{e}+w / 2}{\sqrt{\Delta r\left(2 R_{0}-\Delta r\right)+\left(r_{c}+r_{e}+w / 2\right)^{2}}}$

When $\Delta L_{p}=0$, the hoop strain at the inner side of the hollow groove is 0 . And when $\Delta L_{p}=w+2 r_{e}+\Delta l$, the hoop strain at the outer side of the hollow groove is $\varepsilon_{\theta-\text { out }}$.

In this draw-in event, it can be seen as plane strain state $\left(d \varepsilon_{z}=0\right)$. So the effective strain can be given as 


$$
\bar{\varepsilon}_{d}=\sqrt{\frac{2(1+\bar{R})}{2 \bar{R}+1}}\left(-\varepsilon_{\theta}\right)
$$

Next, the stretching will be considered. It is assumed that the metals inside of the hollow groove are stretched uniformly, i.e. have uniform radial strain. And since the metals are pushed upwards, it is reasonable to think that the metals have no hoop strain change ( $d \varepsilon_{\theta}=0$ ) during the stretching event. Suppose the metals in the hollow groove are stretched to have a radial length $L$, the radial strain of the metal "bubble" can be given as

$$
\varepsilon_{r}=\ln \left(\frac{L}{w+2 r_{e}+\Delta l}\right)
$$

So the effective strain can be given as

$$
\bar{\varepsilon}_{s}=\frac{1+\bar{R}}{\sqrt{1+2 \bar{R}}} \varepsilon_{r}
$$

Therefore, because of the electromagnetic pulse, the metals are drawn and stretched to form a "bubble”. And the radial strain and the hoop strain are increased. The changes can be given as Eq. (4.29) and (4.31).

The total improvement of the effective strain can be estimated by $\bar{\varepsilon}^{e}=\bar{\varepsilon}_{d}+\bar{\varepsilon}_{s}$

i.e. $\bar{\varepsilon}^{e}=\sqrt{\frac{2(1+\bar{R})}{2 \bar{R}+1}}\left(-\varepsilon_{\theta}\right)+\frac{1+\bar{R}}{\sqrt{1+2 \bar{R}}} \varepsilon_{r}$ 


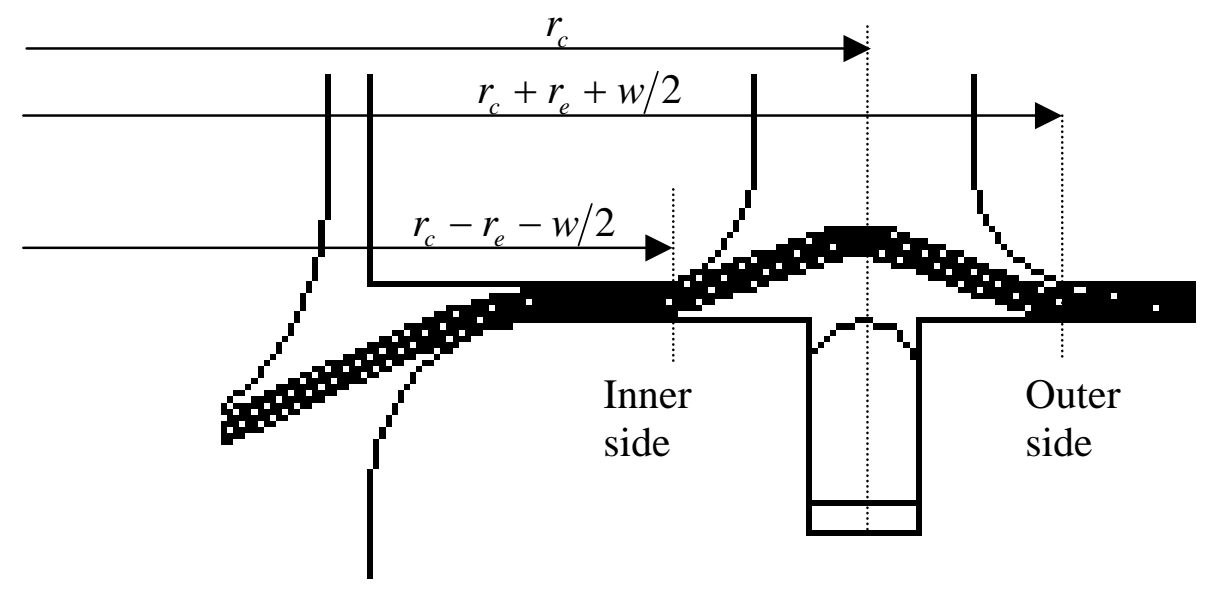

Figure 4.28 Partial schematic of stretching and draw-in caused by electromagnetic pulse

Above analysis assumes hat the draw-in and the stretching happen sequentially. While, actually they should happen simultaneously. Analyzing the stress states will be helpful to determine the relationship between the stretching and the draw-in.

The flange outside of the hollow groove can be seen same as the conventional deep drawing. There are three components to resistant the draw-in from the flange into the hollow groove: (1) Plastic deformation due to flange shrink; (2) Friction at the flange rim; (3) Friction at the radius of hollow groove.

Therefore, the radial stress just near the outer side of the hollow groove can be given as

$$
\sigma_{r_{\text {out }}}=\left[K\left[\sqrt{\frac{2(1+\bar{R})}{2 \bar{R}+1}}\right]^{1+n} \int_{r_{c}+r_{e}+w / 2}^{r_{0}}\left(\ln \left(\frac{R}{r}\right)\right)^{n} \frac{d r}{r}+\frac{\mu B}{\pi r_{0} t}\right] \exp \left(\mu \frac{\pi}{4}\right)
$$


where $r_{0}$ is the current radius of blank. $r_{0}=R_{0}-\Delta r$. Since the friction wrap angle is less than $\pi / 2$, here we take it as $\pi / 4$.

Now we get the radial stress at the outer side of the hollow groove. Because the stretching and the draw-in actually happen at same time, the stretching must have the same radial stress as the one given in Eq. (4.33). It is assumed that the stretching is in the plane stress state condition $\left(\sigma_{z}=0\right)$ and the plane strain state condition $\left(d \varepsilon_{\theta}=0\right)$. So the stress and strain states can be given as

$\varepsilon_{r}=\frac{\sqrt{1+2 \bar{R}}}{1+\bar{R}} \bar{\varepsilon}$ and $\sigma_{r}=\frac{K(1+\bar{R})}{\sqrt{1+2 \bar{R}}}(\bar{\varepsilon})^{n}$

From above two equations, the radial stress of the metals in the stretching can be expressed as

$\sigma_{r}=K\left(\frac{1+\bar{R}}{\sqrt{1+2 \bar{R}}}\right)^{1+n}\left[\ln \left(\frac{L}{\left.w+2 r_{e}+\frac{\Delta r\left(2 R_{0}-\Delta r\right)}{2 r_{c}}\right)}\right]^{n}\right.$

Equating (4.33) and (4.34), there is

$K\left(\frac{1+\bar{R}}{\sqrt{1+2 \bar{R}}}\right)^{1+n}\left[\ln \left(\frac{L}{w+2 r_{e}+\frac{\Delta r\left(2 R_{0}-\Delta r\right)}{2 r_{c}}}\right)\right]^{n}=$ 


$$
\left[K\left[\sqrt{\frac{2(1+\bar{R})}{2 \bar{R}+1}}\right]^{1+n} \int_{r_{c}+r_{e}+w / 2}^{R_{0}-\Delta r}\left(\ln \left(\frac{R}{r}\right)\right)^{n} \frac{d r}{r}+\frac{\mu B}{\pi r_{0} t}\right] \exp \left(\mu \frac{\pi}{4}\right)
$$

Solving above equation, we can get a relationship between the draw-in $\Delta r$ and the stretching $L$. But above equation needs numerical method to solve.

It must to point out that in the electromagnetic pulse, the velocity is high, even can reach $200 \mathrm{~m} / \mathrm{s}$. The deformation behavior in high velocity should be different from the one in low velocity. Above analysis is carried out assuming the velocity is low and the radial stresses in the flange and the "bubble" are same. While, in the high velocity situation, the radial stresses in the above two regions may be unequal. So it is likely to add some or even big error to the calculation.

Although above analysis may have errors, it still can give some understanding about the process, and some basic guideline for the process design. To get more accurate calculation results, the deformation behavior in high velocity should be considered.

\subsubsection{Reduction of resistance force}

In conventional deep drawing, one of the four components for the resistance force is caused by plastic deformation due to the flange shrink. While, in electromagnetically assisted deep drawing, instead of the whole flange, only partial of flange will involve the shrink deformation when the punch goes down. As described in last section, because of 
the stretched "bubble", when punch goes down, partial of flange from the die radius to the "bubble" will be drawn towards to the die cavity. The "bubble" will be pulled out. And the rest of the flange outside of the "bubble" will not have deformation until the "bubble" is pulled flat. Then, a new "bubble" will be made by electromagnetic pulse. Therefore, only partial flange involves the shrink deformation during the movement of punch, which reduces the drawing force transmitted through the cup wall. And because the outer edge of the blank doesn't move during the punch movement, there is no friction force at the flange rim for the resistance force.

So for electromagnetically assisted deep drawing, there are only three components of resistance force:

(1) Plastic deformation due to partial flange shrink;

(2) Plastic deformation due to bending and unbending at the die radius;

(3) Friction at the die radius;

For the first component, there are two different regions. One is electromagnetically stretched region, which has extra stretching strain and increased flow stress. Another one is unaffected region, which behaves same as the conventional deep drawing.

Similar as the conventional deep drawing, the stress which needs to transmit in the cup wall to draw the flange into die cavity can be written as

$$
{\sigma_{r_{c 0}}}^{e}=\left({\sigma_{r_{c d}}}^{e}\right) \exp \left(\mu \frac{\pi}{2}\right)+\sigma_{w}
$$


i.e. $\quad \sigma_{r_{c 0}}{ }^{e}=\left[K \sqrt{\frac{2(1+\bar{R})}{2 \bar{R}+1}} \int_{r_{c d}}^{\left(r_{c}-\frac{w}{2}-r_{e}\right)}\left(\bar{\varepsilon}_{i}^{e}+\bar{\varepsilon}\right)^{n} \frac{d r}{r}\right] \exp \left(\mu \frac{\pi}{2}\right)+\frac{\sigma_{b} t}{2 r_{d}+t}$

where $\bar{\varepsilon}_{i}^{e}$ is the effective strain caused by electromagnetic pulses, $\bar{\varepsilon}$ is the effective strain caused by the flange shrink.

And the drawing force can be written as

$$
F^{e}=2 \pi r_{c 0} t_{0}\left[\left[K \sqrt{\frac{2(1+\bar{R})}{2 \bar{R}+1}} \int_{r_{c d}}^{\left(r_{c}-\frac{w}{2}-r_{e}\right)}\left(\bar{\varepsilon}_{i}^{e}+\bar{\varepsilon}\right)^{n} \frac{d r}{r}\right] \exp \left(\mu \frac{\pi}{2}\right)+\frac{\sigma_{b} t}{2 r_{d}+t}\right]
$$

In electromagnetically assisted deep drawing, the radial drawing stress in cup wall also should always lower than the critical drawing stress. By creating the partial flange shrink, it is possible to keep the radial drawing stress lower than the critical drawing stress.

\subsubsection{Analysis step by step}

We will start the analysis from the first beginning of the electromagnetically assisted deep drawing step by step.

First electromagnetic pulse Before the punch goes down, the first electromagnetic pulse is given to make the first "bubble". Assuming the metals inside the hollow groove are stretched to have the radial length ( $L^{1}$ ) and the blank radius is reduced by $\Delta r^{1}$. From Eqs. (4.28) and (4.30), the radial strain and the hoop strain can be given as

$$
\varepsilon_{r}^{1}=\ln \left(\frac{L^{1}}{w+2 r_{e}+\Delta l^{1}}\right)
$$




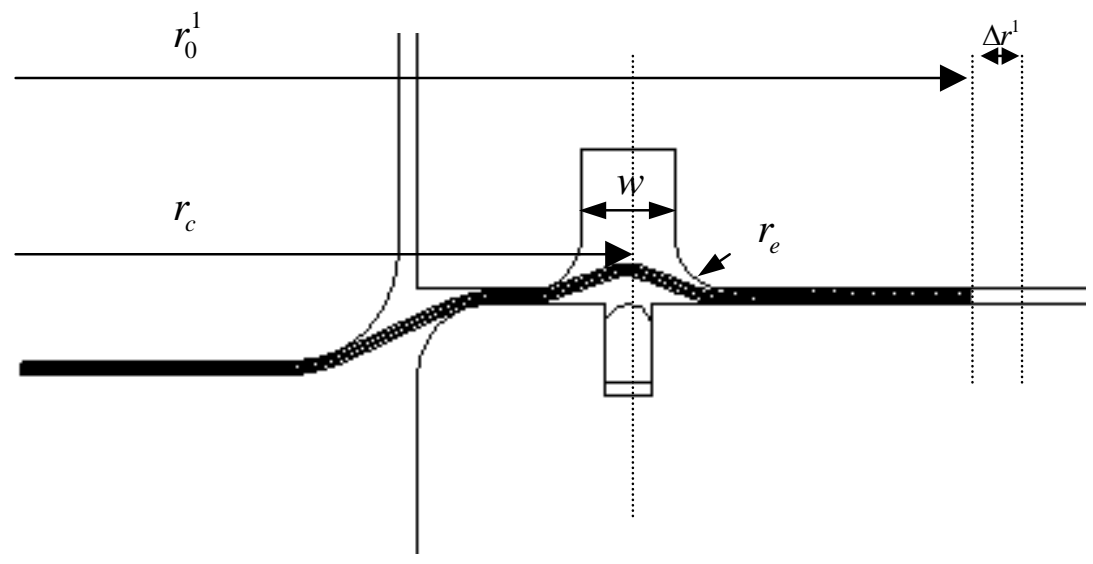

Figure 4.29 Partial drawn cup of electromagnetically assisted deep drawing

$\varepsilon_{\theta}^{1}=\frac{\Delta L_{P}{ }^{1}}{w+2 r_{e}+\Delta l^{1}} \ln \frac{r_{c}+r_{e}+w / 2}{\sqrt{\Delta r^{1}\left(2 R_{0}-\Delta r^{1}\right)+\left(r_{c}+r_{e}+w / 2\right)^{2}}}$

where $\Delta l^{1}=\frac{\Delta r^{1}\left(2 R_{0}-\Delta r^{1}\right)}{2 r_{c}}, \Delta L_{p}^{1}$ is the relative line distance to the inner side of the hollow groove.

And the improvement of the radial length can be given as

$$
\Delta L^{1}=L^{1}-\left(w+2 r_{e}\right)=\left(w+2 r_{e}+\Delta l^{1}\right) \exp \left(\varepsilon_{r_{b}}^{1}\right)-\left(w+2 r_{e}\right)
$$

The effective strain can be expressed as

$$
\bar{\varepsilon}_{1}^{e}=\sqrt{\frac{2(1+\bar{R})}{2 \bar{R}+1}}\left(-\varepsilon_{\theta}^{1}\right)+\frac{1+\bar{R}}{\sqrt{1+2 \bar{R}}} \varepsilon_{r}^{1}
$$


First punch stroke After the first electromagnetic pulse, the punch goes down and draws metals from the flange into the die cavity. The flange from the die radius to the inner side of the hollow groove (unaffected electromagnetically) will behave same as the conventional deep drawing and be drawn towards the die cavity.

Because of the material continuity, the stretched "bubble" region will be pulled out. It can be considered as the material is drawn inward with continuous supply from the "bubble" formed during earlier electromagnetic pulse. There are three points worth to mention again here:

(1) The "bubble" creates the geometry-discontinuity of the flange. When the punch draws the flange into die cavity, the metals can be considered to be free to flow out from the hollow groove. And the flange outside of the hollow groove doesn't involve the deformation until the bubble is pulled flat during the punch movement.

(2) Since it is stretched already and gets radial and hoop strains, the metal from the "bubble" gets the increased effective strain. Except the increased effective strain, this region will behave same as the region unaffected electromagnetically.

(3) When the "bubble" is pulled flat, the punch should stop to keep the condition of the partial flange shrink condition. Otherwise, large drawing force will occur, which leads to the tearing at the cup wall.

So according to the volume constancy, the maximum punch stroke can be given as $2 \pi r_{c 0} \Delta h_{\max }{ }^{1}=2 \pi r_{c} \Delta L^{1}$, 
i.e. $\Delta h_{\max }^{1}=\frac{r_{c}}{r_{c 0}} \Delta L^{1}$

Therefore, to keep the condition of partial flange shrink, the punch stroke $\Delta h^{1}$ should be set to less than $\left(\frac{r_{c}}{r_{c 0}} \Delta L^{1}\right)$.

To calculate the stress in the flange, it is assumed that there is no thickness change, i.e. it's in plane strain state $\left(d \varepsilon_{z}=0\right)$. And the blank is also in the plane stress state $\left(\sigma_{z}=0\right)$.

So the effective strain $\bar{\varepsilon}$ in the flange region is evaluated from plane strain deformation $\left(d \varepsilon_{z}=0\right)$ and incompressibility conditions from Eq. (4.8) as

$\bar{\varepsilon}^{1}=\sqrt{\frac{2(1+\bar{R})}{2 \bar{R}+1}}\left(-\varepsilon_{\theta}\right)+\bar{\varepsilon}_{1}^{e}$

where the hoop strain in the flange region is $-\varepsilon_{\theta}=\ln (R / r) . R$ is the initial radius of the annular ring which reaches radius $r$ after the cup has been partially drawn. And $\bar{\varepsilon}_{1}^{e}$ is given in Eq. (4.41) for the region that the metals flow from the "bubble", and is zero for the region unaffected electromagnetically.

From Eq. (4.6) and (4.12), the effective stress in the flange can be obtained as

$$
\bar{\sigma}^{1}=K\left[\sqrt{\frac{2(1+\bar{R})}{2 \bar{R}+1}} \ln \left(\frac{R}{r}\right)+\bar{\varepsilon}_{1}^{e}\right]^{n}
$$


Using Eqs. (4.11), (4.12) and (4.14) and integrating from the die radius $\left(r_{c d}\right)$ to the inner side of hollow groove $\left(r_{c}-r_{e}-w / 2\right)$, the radial drawing stress at radius $r_{c d}$ in the flange can be obtained as

$$
\int_{r_{c d}}^{r_{c}-r_{e}-w / 2} d \sigma_{r}=-K \sqrt{\frac{2(1+\bar{R}}{2 \bar{R}+1}} \int_{r_{c d}}^{r_{c}-r_{e}-w / 2}\left[\sqrt{\frac{2(1+\bar{R}}{2 \bar{R}+1}} \ln \left(\frac{R}{r}\right)+\bar{\varepsilon}_{1}^{e}\right]^{n} \frac{d r}{r}
$$

The above equation can be written as

$$
\sigma_{r_{c d}}^{1}=K \sqrt{\frac{2(1+\bar{R}}{2 \bar{R}+1}} \int_{r_{c d}}^{r_{c}-r_{e}-w / 2}\left[\sqrt{\frac{2(1+\bar{R}}{2 \bar{R}+1}} \ln \left(\frac{R}{r}\right)+\bar{\varepsilon}_{1}^{e}\right]^{n} \frac{d r}{r}
$$

Or above equation can be written as

$$
\sigma_{r_{c d}}{ }^{1}=K\left[\sqrt{\frac{2(1+\bar{R}}{2 \bar{R}+1}}\right]^{1+n} \int_{r_{c d}}^{r_{u}^{1}}\left[\ln \left(\frac{R}{r}\right)\right]^{n} \frac{d r}{r}+K \sqrt{\frac{2(1+\bar{R}}{2 \bar{R}+1}} \int_{r_{u}^{1}}^{r_{c}-r_{e}-w / 2}\left[\sqrt{\frac{2(1+\bar{R}}{2 \bar{R}+1}} \ln \left(\frac{R}{r}\right)+\bar{\varepsilon}_{1}^{e}\right]^{n} \frac{d r}{r}
$$

where $r_{u}^{1}$ is the current radius of the region unaffected electromagnetically.

We can express $r_{u}{ }^{1}$ as a function of the punch displacement $h$ as following

$$
r_{u}^{1}(h)=\sqrt{\left(r_{c}-r_{e}-w / 2\right)^{2}-2 r_{c 0} h}
$$

As described before, there are still two more components contributed to the resistance force. One is the friction at the die radius, another one is the plastic deformation bending 
and unbending at the die radius. Therefore, the radial stress at the cup wall during the punch movement can be given as

$$
\begin{aligned}
\sigma_{r_{c 0}}{ }^{1}= & \left(K\left[\sqrt{\frac{2(1+\bar{R}}{2 \bar{R}+1}}\right]^{1+n} \int_{r_{c d}}^{r_{u}^{1}}\left[\ln \left(\frac{R}{r}\right)\right]^{n} \frac{d r}{r}+K \sqrt{\frac{2(1+\bar{R}}{2 \bar{R}+1}} \int_{r_{u}^{1}}^{r_{c}-r_{e}-w / 2}\left[\sqrt{\frac{2(1+\bar{R}}{2 \bar{R}+1}} \ln \left(\frac{R}{r}\right)+\bar{\varepsilon}_{1}^{e}\right]^{n} \frac{d r}{r}\right)^{n} \\
& \cdot \exp \left(\mu \frac{\pi}{2}\right)+\frac{\sigma_{b} t_{0}}{2 r_{d}+t_{0}}
\end{aligned}
$$

Because the punch goes down, the "bubble" inside of the hollow groove is pulled out and the radial line length is reduced. We can derive the relationship of the punch stroke $\Delta h^{1}$ and the reduction of the radial line length of the "bubble” $\Delta L_{q}^{1}$ as following

$$
\begin{aligned}
& 2 \pi r_{c 0} \Delta h^{1}=2 \pi r_{c} \Delta L_{q}^{1}, \\
& \text { i.e. } \Delta L_{q}^{1}=\frac{r_{c 0}}{r_{c}} \Delta h^{1}
\end{aligned}
$$

After the first punch stroke, the punch displacement is $h=\Delta h^{1}$. The radial line length of the "bubble" reduces to $\left(L^{1}-\Delta L_{q}^{1}\right)$. The radius of the religion unaffected electromagnetically reduces to $r_{u}{ }^{1}$. And the hoop strain at the inner side of the hollow groove becomes

$$
\varepsilon_{\theta-i n}^{1}=\frac{\Delta L_{q}^{1}}{w+2 r_{e}+\Delta l^{1}} \ln \frac{r_{c}+r_{e}+w / 2}{\sqrt{\Delta r^{1}\left(2 R_{0}-\Delta r^{1}\right)+\left(r_{c}+r_{e}+w / 2\right)^{2}}}+\ln \frac{r_{c}-r_{e}-w / 2}{\Delta L_{q}^{1}+r_{c}-r_{e}-w / 2}
$$

ith electromagnetic pulse After the ith electromagnetic pulse, the metals inside of the hollow groove is stretched to have line length $\left(L^{i}\right)$ and the edge radius of blank is 
reduced by $\Delta r^{i}$. From Eqs. (4.28) and (4.30), the radial strain and the hoop strain can be given as

$\varepsilon_{r}^{i}=\ln \left(\frac{L^{i}}{L^{i-1}-\Delta L_{q}^{i-1}+\Delta l^{i}}\right)+\varepsilon_{r}^{i-1}$

$\varepsilon_{\theta}^{i}=\frac{\Delta L_{P}{ }^{i}}{w+2 r_{e}+\Delta l^{i}} \ln \frac{r_{c}+r_{e}+w / 2}{\sqrt{\Delta r^{i}\left(2 R_{0}-\Delta r^{i}\right)+\left(r_{c}+r_{e}+w / 2\right)^{2}}}+\varepsilon_{\theta}^{i-1}$

where $\Delta l^{i}=\frac{\Delta r^{i}\left(2 R_{0}-\Delta r^{i}\right)}{2 r_{c}}, \Delta L_{p}^{i}$ is the relative line distance to the inner side of the hollow groove.

And the improvement of the radial length can be given as

$$
\Delta L^{i}=L^{i}-\left(w+2 r_{e}\right)=\left(L^{i-1}-\Delta L_{q}^{i-1}+\Delta l^{i}\right) \exp \left(\varepsilon_{r}^{i}-\varepsilon_{r}^{i-1}\right)-\left(w+2 r_{e}\right)
$$

The effective strain can be expressed as

$$
\bar{\varepsilon}_{i}^{e}=\sqrt{\frac{2(1+\bar{R})}{2 \bar{R}+1}}\left(-\varepsilon_{\theta}^{i}\right)+\frac{1+\bar{R}}{\sqrt{1+2 \bar{R}}} \varepsilon_{r}^{i}
$$

ith punch stroke After the ith electromagnetic pulse, the ith punch stroke will be carried out to draw the metals into the die cavity. To keep the partial flange shrink condition, the maximum punch stroke can be given as

$$
\begin{aligned}
& 2 \pi r_{c 0} \Delta h_{\max }{ }^{i}=2 \pi r_{c} \Delta L^{i}, \\
& \text { i.e. } \Delta h_{\max }^{i}=\frac{r_{c}}{r_{c 0}} \Delta L^{i}
\end{aligned}
$$


The punch stroke $\Delta h^{i}$ should be set to less than $\left(\frac{r_{c}}{r_{c 0}} \Delta L^{i}\right)$. While, $\Delta h^{i}$ should not be too small, which will cost too much time. To set the value of $\Delta h^{i}$, the efficiency of the whole process and the partial flange shrink should both be considered.

Similar to first punch stroke, the effective strain $\bar{\varepsilon}$ in the flange region is evaluated from plane strain deformation ( $\left.d \varepsilon_{z}=0\right)$ and incompressibility conditions from Eq. (4.8) as

$\bar{\varepsilon}^{i}=\sqrt{\frac{2(1+\bar{R})}{2 \bar{R}+1}}\left(-\varepsilon_{\theta}\right)+\bar{\varepsilon}_{i}^{e}$

where the hoop strain in the flange region is $-\varepsilon_{\theta}=\ln (R / r) . R$ is the initial radius of the annular ring which reaches radius $r$ after the cup has been partially drawn. And $\bar{\varepsilon}_{i}^{e}$ is given in Eq. (4.54) for the region that the metals flow from the "bubble”.

The effective stress in the flange can be obtained as

$$
\bar{\sigma}^{i}=K\left[\sqrt{\frac{2(1+\bar{R})}{2 \bar{R}+1}} \ln \left(\frac{R}{r}\right)+\bar{\varepsilon}_{i}^{e}\right]^{n}
$$

Using Eqs. (4.11), (4.12) and (4.14) and integrating from the die radius $\left(r_{c d}\right)$ to the inner side of hollow groove $\left(r_{c}-r_{e}-w / 2\right)$, the radial drawing stress at radius $r_{c d}$ in the flange can be obtained as

$$
\int_{r_{c d}}^{r_{c}-r_{e}-w / 2} d \sigma_{r}=-K \sqrt{\frac{2(1+\bar{R}}{2 \bar{R}+1}} \int_{r_{c d}}^{r_{c}-r_{e}-w / 2}\left[\sqrt{\frac{2(1+\bar{R}}{2 \bar{R}+1}} \ln \left(\frac{R}{r}\right)+\bar{\varepsilon}_{i}^{e}\right]^{n} \frac{d r}{r}
$$


Above equation can be written as

$$
\begin{aligned}
\sigma_{r_{c d}}{ }^{i}= & K\left[\sqrt{\frac{2(1+\bar{R}}{2 \bar{R}+1}}\right]^{1+n} \int_{r_{c d}}^{r_{u}^{1}}\left[\ln \left(\frac{R}{r}\right)\right]^{n} \frac{d r}{r}+\sum_{2}^{i-1} K \sqrt{\frac{2(1+\bar{R}}{2 \bar{R}+1}} \int_{r_{u}^{i}}^{r_{u}^{i+1}}\left[\sqrt{\frac{2(1+\bar{R}}{2 \bar{R}+1}} \ln \left(\frac{R}{r}\right)+\bar{\varepsilon}_{i}^{e}\right]^{n} \frac{d r}{r} \\
& +K \sqrt{\frac{2(1+\bar{R}}{2 \bar{R}+1}} \int_{r_{u}^{i}}^{r_{c}-r_{e}-w / 2}\left[\sqrt{\frac{2(1+\bar{R}}{2 \bar{R}+1}} \ln \left(\frac{R}{r}\right)+\bar{\varepsilon}_{i}^{e}\right]^{n} \frac{d r}{r}
\end{aligned}
$$

where $r_{u}^{i}$ is the current radius of the regions affected by electromagnetic pulses.

We can express $r_{u}{ }^{i}$ as a function of the punch displacement $\Delta h^{i}$ as following

$$
r_{u}^{i}(h)=\sqrt{\left(r_{c}-r_{e}-w / 2\right)^{2}-2 r_{c 0} \Delta h^{i}}
$$

Therefore, the radial stress at the cup wall during the punch movement can be given as

$$
\sigma_{r_{c 0}}^{i}=\left(\sigma_{r_{c d}}^{i}\right) \cdot \exp \left(\mu \frac{\pi}{2}\right)+\frac{\sigma_{b} t_{0}}{2 r_{d}+t_{0}}
$$

We can have the relationship of the punch stroke $\Delta h^{i}$ and the reduction of the radial line length of the "bubble" $\Delta L_{q}^{i}$ as following

$$
\begin{aligned}
& 2 \pi r_{c 0} \Delta h^{i}=2 \pi r_{c} \Delta L_{q}^{i}, \\
& \text { i.e. } \Delta L_{q}^{i}=\frac{r_{c 0}}{r_{c}} \Delta h^{i}
\end{aligned}
$$


After the ith punch stroke, the punch displacement is $h=\sum_{1}^{i} \Delta h^{i}$. The radial line length of the "bubble" reduces to $\left(L^{i}-\Delta L_{q}^{i}\right)$. The radius of the religion unaffected electromagnetically reduces to $r_{u}{ }^{1}$. And the hoop strain at the inner side of the hollow groove becomes

$$
\varepsilon_{\theta-i n}^{i}=\frac{\Delta L_{q}^{i}}{w+2 r_{e}+\Delta l^{i}} \ln \frac{r_{c}+r_{e}+w / 2}{\sqrt{\Delta r^{i}\left(2 R_{0}-\Delta r^{i}\right)+\left(r_{c}+r_{e}+w / 2\right)^{2}}}+\ln \frac{r_{c}-r_{e}-w / 2}{\Delta L_{q}^{i}+r_{c}-r_{e}-w / 2}+\varepsilon_{\theta}^{i-1}
$$

\subsubsection{Wrinkling and buckling}

Wrinkling and buckling will occur when there is too much compression [102-107]. For conventional deep drawing, there is only wrinkling at the rim of the flange. While for electromagnetically assisted deep drawing, besides the wrinkling, there is also buckling may occur inside of the hollow groove.

Increasing clamp load is an effective way to prevent wrinkling for both conventional and electromagnetically assisted deep drawing. The classic deep drawing analysis [12] provides an equation below to estimate the maximum hoop strain for wrinkling without clamp load

$$
\varepsilon_{\theta} \leq \frac{n}{0.544}\left(\frac{t}{R-r_{c 0}}\right)
$$

where $n$ is the strain hardening index, $t$ is the thickness of the sheet, $r_{c 0}$ is the punch radius and $R$ is the current radius of the outer edge of the blank. 
To prevent wrinkling, the minimum clamp load is given as [12]

$B=0.195 K \frac{\pi r_{c 0}^{2}}{4} F(B)$

where $K$ is the strength coefficient, $F(B)$ is a function of the material properties and the ratio of $R_{0} / r_{c 0}$.

For the buckling inside of the hollow groove, it is difficult to determine the critical compression by classical analysis. The metals inside of the hollow groove are curved and are clamped at the two ends. There is no such analysis in the literature [102-107]. The exact expression for the critical compression will not be discussed in this dissertation.

Some qualitative rules can be given here. Similar to the wrinkling at the edge of the flange, the critical hoop strain must be relative to the material properties $n$, the current thickness of the blank $t$, the radial line length of metals inside of the hollow groove $L^{i}$, the width of the hollow groove $\left(w+2 r_{e}\right)$ and the clamp load $B$. It is reasonable to get that the thinner thickness and the larger radial line length will make it easier to buckle. And the larger clamp load will delay buckling.

Since there is no clamp force to apply on the metals inside of the hollow groove, it is actually difficult to control the buckling in this region. One possible method is to decrease ratio of groove width to the sheet thickness, which can increase the ability to resist buckling. Another possible method is to reduce the hoop strain, which will limit the deformation. The specific way to use depends on the practical problems. 
For example, in the electromagnetically assisted deep drawing of the very large blank, there is almost no draw-in from the outside of the hollow groove, which makes the metals inside of the hollow groove have small hoop strain. Although they are stretched very much and the radial strain can even be 53\%, there is almost no buckling for this kind of forming process.

\subsubsection{Summary}

Above analysis show the basic rules for the electromagnetically assisted deep drawing.

(1) The amount of the stretching $L$ and the draw-in $\Delta r$ has some relationship for a certain blank size and certain energy level. The bigger the blank size, the smaller the draw-in $\Delta r$. The stretching will thin the sheet thickness. So it is better to get a bigger draw-in.

(2) According to the stretching and the draw-in, the punch stroke should be set to keep the radial drawing stress lower than the critical drawing stress. And the punch stroke should not be too small, which will cost much processing time and waste the electromagnetic energy.

(3) Except wrinkling, buckling is likely to happen inside of the hollow groove which will cause failure. But if the metals inside of the hollow groove will not be included in the final part, electromagnetic pulse could eliminate the buckling once it happens. Then the metals can continue to flow inwards, until the buckling happens again which needs electromagnetic pulse to eliminate.

(4) It is difficult to estimate the stress and strain for electromagnetically assisted deep drawing, since it is dynamic process and depends on lots of parameters. Above 2D 
analytical model is complex to use in pencil-and-paper way. While it can provide a better understanding of this process.

\subsubsection{Electromagnetic pulse design}

\subsubsection{Geometry of coil and hollow groove}

The geometry of the electromagnetic coil is very important, which determines the drawing force in electromagnetically assisted deep drawing. As shown in Eq. (4.58), only the region from the die radius to the inner side of the hollow groove will involve the flange shrink when the punch moves down. So the large coil radius makes more metals in the flange involve with the flange shrink, which will increase the drawing force in the cup wall and cause tearing. But the small coil radius makes the draw-in difficult, and then can’t draw more metals from the outer flange.

So a suitable coil radius is needed to make draw-in easier and meanwhile keep drawing force small. Generally, the ratio of the coil radius and the punch radius should be smaller than the LDR. In this experiment, the LDR of the $\mathrm{Al} 2219-\mathrm{O}$ is 1.9. And the ratio of the coil radius and the punch radius is 1.6.

The coil width should be carefully determined. Large coil width will make too much metal inside of the hollow groove and increase the potential of buckling. But small coil width makes too little metal to stretch and leads to low efficiency. Therefore, coil width is also very important. In this experiment, the coil width was set to be $3.17 \mathrm{~mm}$. 
The hollow groove is set to be twice of the coil width. And the edge radius $r_{e}$ of the hollow groove is set to be $3.17 \mathrm{~mm}$. The basic rule for it is to make the metal "bubble" flow out easily.

\subsubsection{Electromagnetic energy level}

As described in last section, electromagnetic pulses cause stretching and draw-in to make a "bubble". There must be some relationship between stretching, and draw-in and electromagnetic energy. The higher energy level is, the bigger stretching and draw-in is. Generally, the amount of the stretching after electromagnetic pulse should be determined first. Then according to different blank size, the amount of draw-in can be derived. At last, the electromagnetic energy can be decided from the required deformation pressure. Above is the basic procedure about the determination of electromagnetic energy level.

Eq. (4.35) shows the relationship between stretching and draw-in, shown in. The goal of this analysis is to find the relationship between the stretching and the electromagnetic energy. 


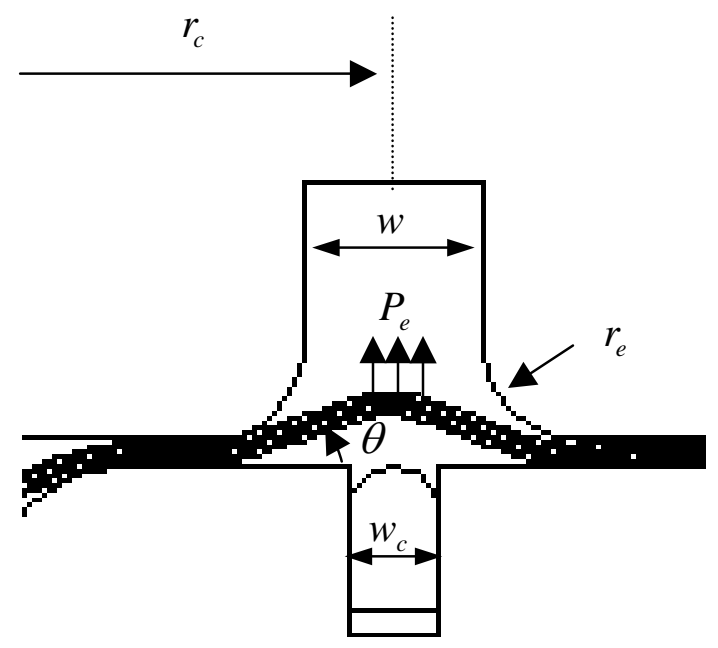

Figure 4.30 Schematic of electromagnetic forces

Figure 4.30 shows the schematic of electromagnetic forces applying on the metals inside of the hollow groove. Assuming the stretched "bubble" has the radial line length $L^{i}$. The angle $\theta$ can be expressed as

$\theta=\cos ^{-1}\left(\frac{w+2 r_{e}}{L^{i}}\right)$

According to the balance of the forces, there is

$F_{e}=2 \sin \theta \cdot \sigma_{r} 2 \pi\left(r_{c}+w / 2\right) t$

And $F_{e}=2 \pi r_{c} w_{c} p_{e}$

where $P_{e}$ is the electromagnetic pressure on the metal sheet. 
During the stretching, it is assumed that the metals are in the plane strain state $\left(d \varepsilon_{\theta}=0\right)$ and the plane stress state $\left(\sigma_{z}=0\right)$. Therefore, the radial stress can be given as

$$
\begin{aligned}
& \sigma_{r}^{i}=K\left(\frac{1+\bar{R}}{\sqrt{1+2 \bar{R}}}\right)^{1+n}\left(\varepsilon_{r}^{i}\right)^{n} \\
& \varepsilon_{r}^{i}=\ln \left(\frac{L^{i}}{L^{i-1}-\Delta L_{q}^{i-1}+\Delta l^{i}}\right)+\varepsilon_{r}^{i-1}
\end{aligned}
$$

where $\Delta l^{i}=\frac{\Delta r^{i}\left(2 R_{0}-\Delta r^{i}\right)}{2 r_{c}}$. So from Eqs. (4.65) (4.69), the electromagnetic pressure can be expressed as

$$
P_{e}=\frac{2\left(r_{c}+w / 2\right) t}{r_{c} w_{c}} \sin \left(\cos ^{-1}\left(\frac{w+2 r_{e}}{L^{i}}\right)\right) K\left(\frac{1+\bar{R}}{\sqrt{1+2 \bar{R}}}\right)^{1+n}\left(\ln \left(\frac{L^{i}}{L^{i-1}-\Delta L_{q}^{i-1}+\Delta l^{i}}\right)+\varepsilon_{r}^{i-1}\right)^{n}
$$

After the electromagnetic pressure is determined, the required electromagnetic energy can be derived. The accurate calculation of the dynamic electromagnetic pressure is still in active research and needs complex calculation, which is beyond the scope of this dissertation. Here only some basic estimate equations will be presented.

The electromagnetic pressure between the coil and the blank can be estimated as [59]

$$
P_{e}=\frac{\mu_{0} I_{1}{ }^{\max } I_{2}{ }^{\max }}{2 \pi d w_{c}}
$$

where $I_{1}{ }^{\max }$ and $I_{2}{ }^{\max }$ are the maximum values of the primary current in the coil and the induced current in the blank, $d$ is the distance between the coil and the blank, $w_{c}$ is the width of the coil. During our estimate procedure, it is assumed that the maximum 114 
electromagnetic pressure happens when the primary and induced current reach their peak values.

Generally, the induced current is always less than the primary current [108-109], and can be estimated as

$$
I_{2}{ }^{\max }=\beta_{e} I_{1}{ }^{\max }
$$

For aluminum alloy blank, $\beta_{e}=0.7 \sim 0.8$; For steel blank, $\beta_{e}=0.4 \sim 0.6$. For our experiment, we will take it as 0.7 [110]. From Eqs. (4.71) and (4.72), the required primary current can be derived.

For an electromagnetic forming process, the governing equations [57] are:

$$
\begin{aligned}
& \frac{d}{d t}\left\{\left(L_{\text {bank }}+L_{\text {coil }}\right) I_{1}-M I_{2}\right\}+\left(R_{\text {bank }}+R_{\text {coil }}\right) I_{1}+Q / C=0 \\
& \frac{d}{d t}\left(L_{2} I_{2}-M I_{1}\right)+R_{2} I_{2}=0
\end{aligned}
$$

The estimate for primary current [109] is given as

$$
\begin{aligned}
& I_{1}(t)=\sqrt{\frac{2 E}{L_{a}}} \frac{e^{-\xi \omega t}}{\sqrt{1-\xi^{2}}} \sin (\omega t) \\
& \text { i.e. } I_{1}^{\max }=\sqrt{\frac{2 E}{L_{a}}} \frac{e^{-\xi \pi / 2}}{\sqrt{1-\xi^{2}}}
\end{aligned}
$$

where $E$ is the electromagnetic energy to deliver, $\xi$ is a factor given as $\xi=\frac{1}{2} R_{a} \sqrt{C / L_{a}}$, and $\omega$ is the nature frequency given by $\omega=\sqrt{1 / L_{a} C} . L_{a}$ is the inductance of the system 115 
which is the sum of the capacitor bank and the coil. $R_{a}$ is the resistance of the coil which is the sum of the capacitor bank and the coil. The inductances and resistances of the capacitor bank and the coil are measurable. And for the coil with simple geometry, the inductance and resistance can be estimated.

Therefore, the electromagnetic energy required to deliver can be derived from Eq. (4.76), if the maximum primary current is estimated from Eqs. (4.71) and (4.72).

\subsubsection{Validation}

Last section gives a procedure to estimate the electromagnetic energy to deliver. In this section, it will be validated by experiment result.

Two Al 2219-O blanks with different initial diameters were deformed by same electromagnetic energy level to make a "bubble" inside of the hollow groove. All the blanks had no deformation prior to the electromagnetic pulses. The electromagnetic energy level was $4.2 \mathrm{~kJ}$. And the mechanical properties of Al 2219-O are given in Table 4.3, and the thickness is $0.8198 \mathrm{~mm}$.

For the electromagnetic forming system, the $48 \mathrm{~kJ}$ capacitor bank was used. In the experiments, 2 of 8 capacitors were used. So the inductance of the capacitor bank module is $0.14 \mu \mathrm{H}$, the resistance of it is $0.0065 \Omega$, and the capacitance is $250 \mu \mathrm{F}$. For the coil, 
the measurement showed that the inductance is $0.31 \mu H$, and the resistance is $0.0019 \Omega$. The experiment results and the calculation results are listed in the below Table 4.6.

\begin{tabular}{|c|c|c|c|c|c|c|c|}
\hline $\begin{array}{c}\text { Blank } \\
\text { diameter }\end{array}$ & $\begin{array}{l}\text { Measured } \\
\text { Length of } \\
\text { "bubble" }\end{array}$ & $\begin{array}{l}\text { Measured } \\
\text { Radius } \\
\text { reduction }\end{array}$ & $\begin{array}{l}\text { Calculate } \\
\qquad \mathrm{d} \varepsilon_{r}{ }^{1}\end{array}$ & $\begin{array}{c}\text { Calculate } \\
\qquad \mathrm{d} \sigma_{r}^{1} \\
(\mathrm{MPa})\end{array}$ & $\begin{array}{c}\text { Calculate } \\
\text { d } P_{e} \\
(\mathrm{MPa})\end{array}$ & $\begin{array}{l}\text { Predicted } \\
I_{1}^{\max } \\
(\mathrm{kA})\end{array}$ & $\begin{array}{c}\text { Predicted } \\
\text { energy } \\
E(\mathrm{~kJ})\end{array}$ \\
\hline $102 \mathrm{~mm}$ & $15.5 \mathrm{~mm}$ & $1.6 \mathrm{~mm}$ & 0.055 & 121 & 38.7 & 62.4 & 2.4 \\
\hline $127 \mathrm{~mm}$ & $14.9 \mathrm{~mm}$ & $0.6 \mathrm{~mm}$ & 0.089 & 133 & 38.9 & 58.6 & 2.1 \\
\hline
\end{tabular}

Table 4.6 the calculation results from the predict model

For above two experiments, the electromagnetic energy used was $4.2 \mathrm{~kJ}$ and the peak primary current in the coil was measured as $96 \mathrm{kA}$. The predicted primary current in both cases are about $60 \%$ of the measured primary current. Both of the predicted energies are about $50 \%$ of the actual energy delivered. It is reasonable that not all the electromagnetic energy is used for plastic deformation. Some will convert to the heat. And some will escape to the surrounding space. Therefore, the predicted EM energy should include wasted energies, i.e. considering an efficiency factor. For these experiments, it seems the efficiency factor should be around 2.

In this model, there are some simplifications and assumptions, which could lead to errors. Accurate results need more complex models. While, above calculation shows that the 
predict model for electromagnetic energy gives reasonable results. So this model still can be used in the initial estimates and can be guideline for the initial design.

\subsection{Design methodology}

The electromagnetic forces can be applied on the flange to stretch the metals and draw in the metals. It can be used for axisymmetric deep drawing cup and the corner side of the stamping. The design procedure is as follows:

(1) According to the sheet metal properties and the forming parameters, design the EM coil diameter. The ratio between the coil diameter and the punch diameter should be less than LDR of the sheet metal;

(2) Design the geometry of the coil and the hollow groove. The width of the coil and hollow groove should be suitable, neither too large nor too small. Large width promotes wrinkling, while small width reduces efficiency;

(3) Decide the desired strain for every EM pulse. Estimate the EM pressure according to Eq. (4.70). And then determine the needed EM energy level by Eq. (4.76);

(4) Design the punch advance procedure by Eq. (4.55). The principle is to advance the punch to pull out the metals in the hollow groove;

(5) Estimate the strain distribution according to Eq. (4.38) Eq. (4.62), and adjust the process parameters to avoid the tearing and the wrinkling.

Above design procedure is only for estimation of the electromagnetically assisted deep drawing. The estimate equations are rough and should only be used for guidance. For accurate design, finite element simulation should be applied. 
There are limits for the application of this method as follows.

(1) The sheet metal should have high conductivity. Metals with low conductivity is not suitable for this method;

(2) The hollow groove in the blank holder promotes the buckling for large compression, which leads to the bad shape;

(3) The die and blank holder need to be modified to accommodate the coil. Also there is insulation requirement for die and blank holder.

\subsection{Conclusion}

In this chapter, an application of electromagnetically assisted sheet stamping in deep drawing was introduced. The electromagnetic forces were applied on the flange of the metal part to draw more metals in and to stretch the metals of the flange. The experiment results showed that this method works. It did draw more metals from the outer edge of the flange, and did stretch the metals above the electromagnetic coil. By the draw-in and stretching, the cup height was dramatically increased.

The deep drawing process is complex. Depending the varied blank sizes, the effects of the electromagnetic forces are different. And the hollow groove area needs more attention to prevent buckling, which may limit the application of electromagnetic forces.

2D theoretical analysis was presented for both conventional and electrometrically assisted deep drawing. The analysis can be used to better understand the forming process, and can be guideline for the initial design. 


\section{CHAPTER 5}

\section{ELECTROMAGNETIC FORMING TO INCREASE DRAWING IN FOR LIP-SKIN PANEL}

Last chapter shows that electromagnetic coil can be embedded into the die to apply electromagnetic forces to help draw metals from the flange into die cavity. In this chapter, another application of this kind function will be introduced for lip-skin panel.

\subsection{Analysis of lip-skin panel production}

The lip skin panel is made of aluminum alloy 2219 with the thickness of 0.090 inches. The final configuration of the part is shown in Figure 5.1, and the part is produced by the double-action tooling.

Much of the cost in producing this part can be directly attributed to the multi-stage forming sequence used to fabricate the part. In order to form the part without failure, four forming stages are used, i.e. lubrication, partial forming to a fixed depth, lubrication removing, and annealing. These four steps are repeated four times sequentially increasing the part depth. This process is quite expensive in terms of the labor hours involved in forming the part and it produces a large inventory of work in progress at any time. 


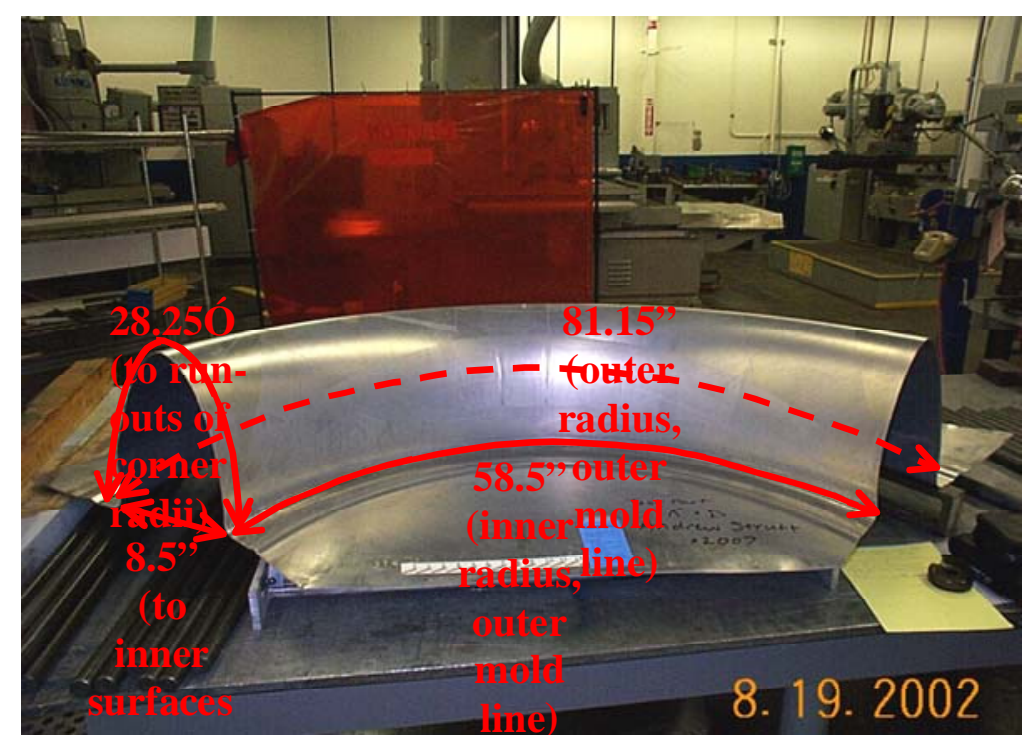

Figure 5.1 Photograph of a completely formed and untrimmed lip skin (The inner and outer arc lengths are noted)

To understand the strain distribution in the conventionally formed part, a standard 2219 blank was etched with a circle grid pattern prior to any deformation, as shown in Figure 5.2. After the production, five sections were cut out from a completely formed part as labeled in Figure 5.2. The major axial strains (nearly coincident with the radial direction on the part) are shown in Figure 5.3. In all cases the strains are near plane strain state. Figure 5.3 shows that the inner part of the panel is challenged both by excessively high strains and possible wrinkling. The inner binder load cannot be increased (promoting tearing) or decreased (promoting wrinkling), so the only available solution is to apply multiple annealing treatments. 

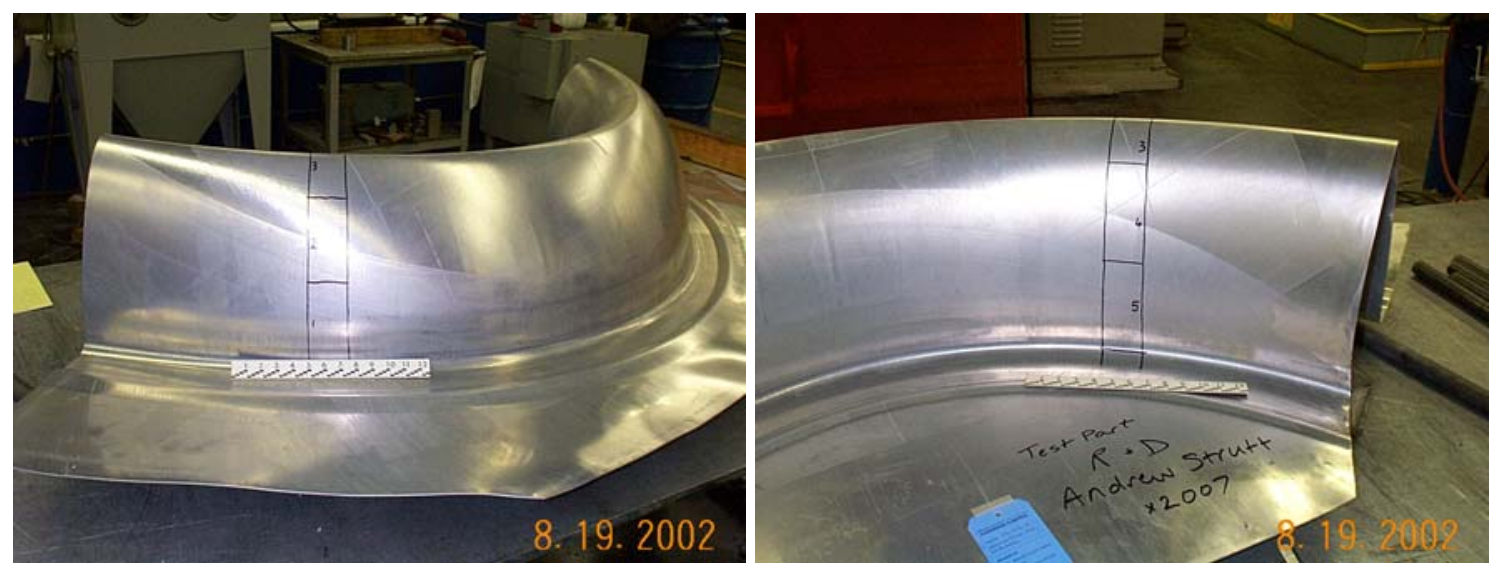

Figure 5.2 Photographs of the grids and formed part with the 5 sub-sections that were cut out labeled

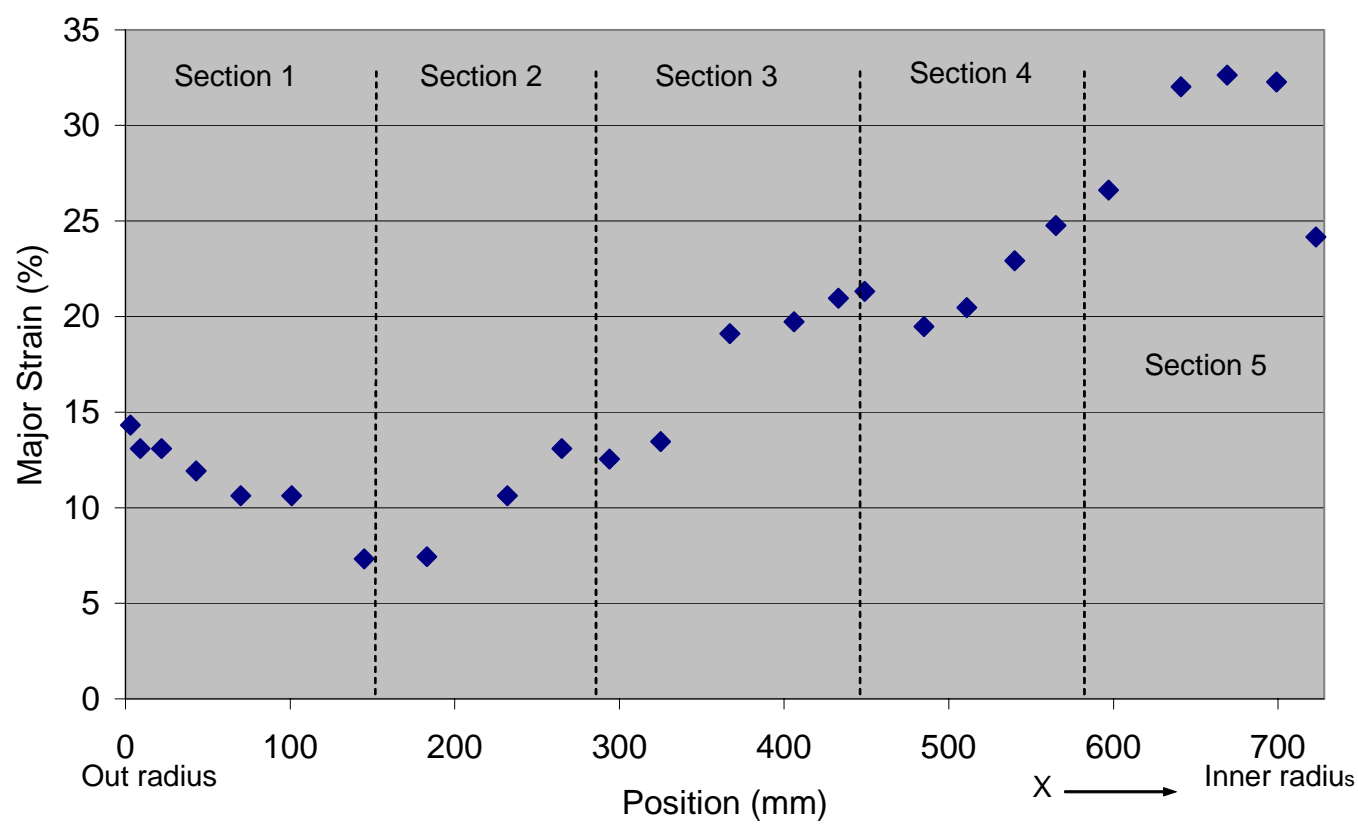

Figure 5.3 Major strains as a function of position for the fully formed lip-skin panel 


\subsection{Design of electromagnetically assisted sheet metal forming}

\subsubsection{Approach design}

As discussed in last section, the draw depth of the lip-skin panel is limited by the tearing and wrinkling. To avoid the tearing and wrinkling, the strain distribution should be carefully controlled to avoid excessive tension and excessive compression. For the lipskin panel, the metal from the flanges should be encouraged to flow into die cavity, which could reduce the tension of the part wall. As shown in last chapter, electromagnetic coil could be applied to draw more metal into die cavity.

The basic approach is to embed electromagnetic coils in the hold-down surface of a prototype tool. Then electromagnetic forces will be applied to encourage draw-in from the flange region to the die cavity without wrinkling or tearing. This approach will be effective, because in conventional forming the only way to encourage draw-in is by increasing the tension in the wall region of the metal part. But the higher tension increases the propensity for tearing. The electromagnetic coils allow the forming energy to be provided away from the highly tensioned region that is near fracture. This will certainly increase the draw depth available in a single stroke.

To verify the feasibility of the electromagnetic assisted sheet metal forming, it was proposed to design a smaller and simplified set of tools and carry out a hybrid hydraulic electromagnetically assisted forming process. The lip-skin panel part and the forming tooling were scaled down about one third of the original metal part and tooling. A 90 degree section would be formed from a blank and would be shaped into a quarter section 
of a nose-lip-like contour. The approximate ratio of the inner and outer radii for the part should be maintained. Therefore, according to the original part, the inner radius of the experiment part was determined to be $360 \mathrm{~mm}$, and the outer one was $500 \mathrm{~mm}$.

\subsubsection{Tooling design}

According to the above approach and the dimensions of hydraulic press in Ohio State University, a scaled-down tooling set was designed and made of steel, shown in below figures.

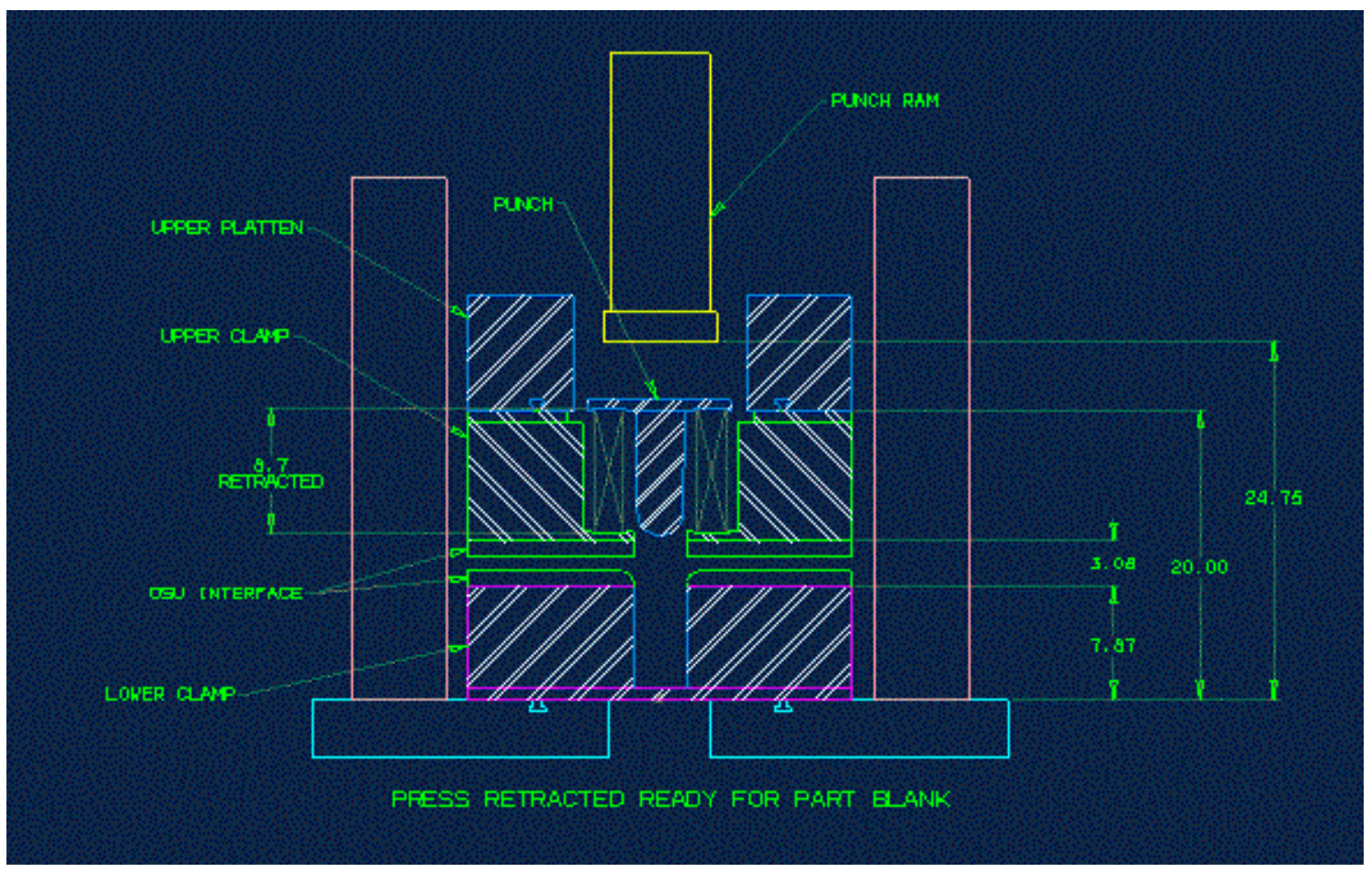

Figure 5.4 Assembly drafts of steel tooling and the press 


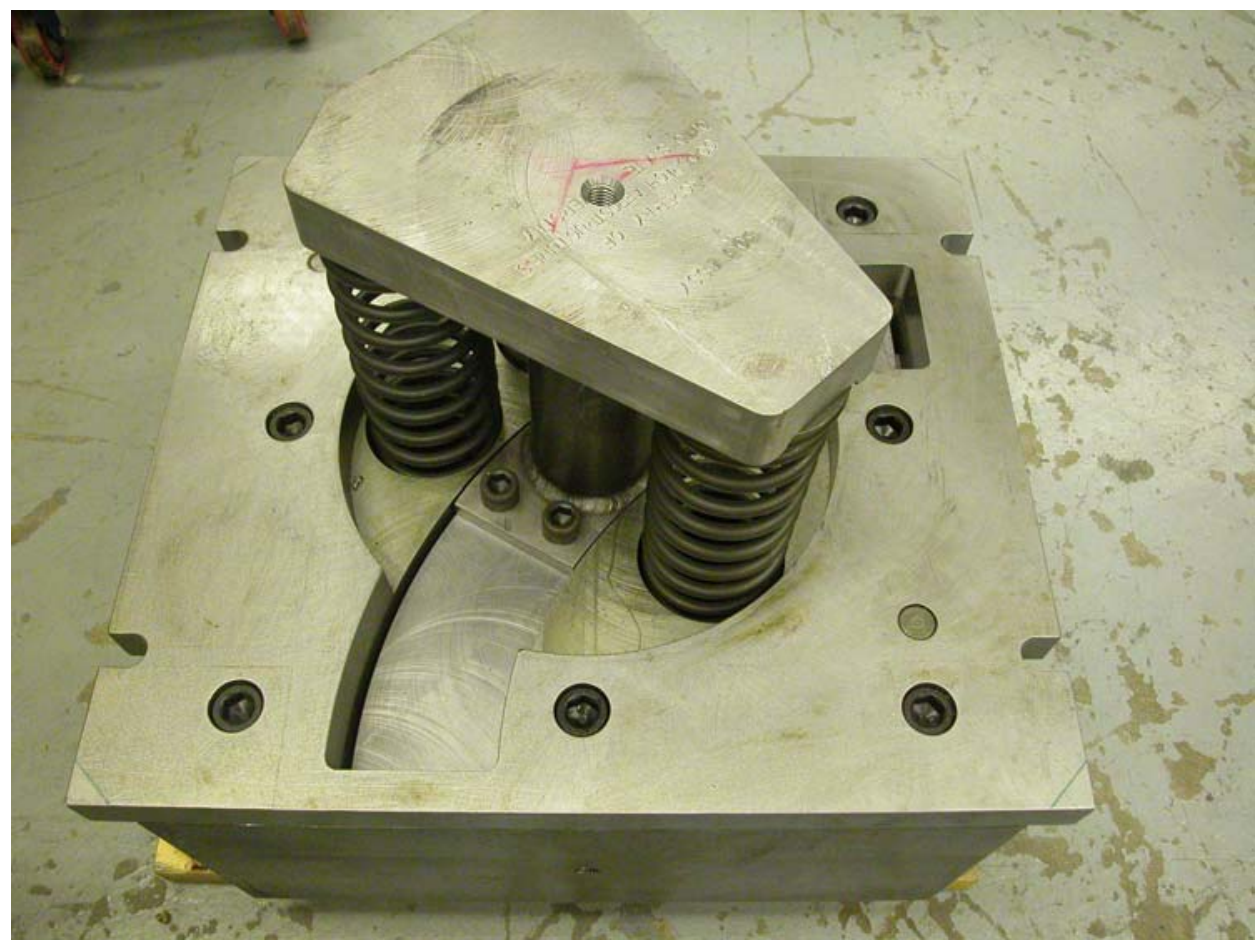

Figure 5.5 Photo of the steel tooling

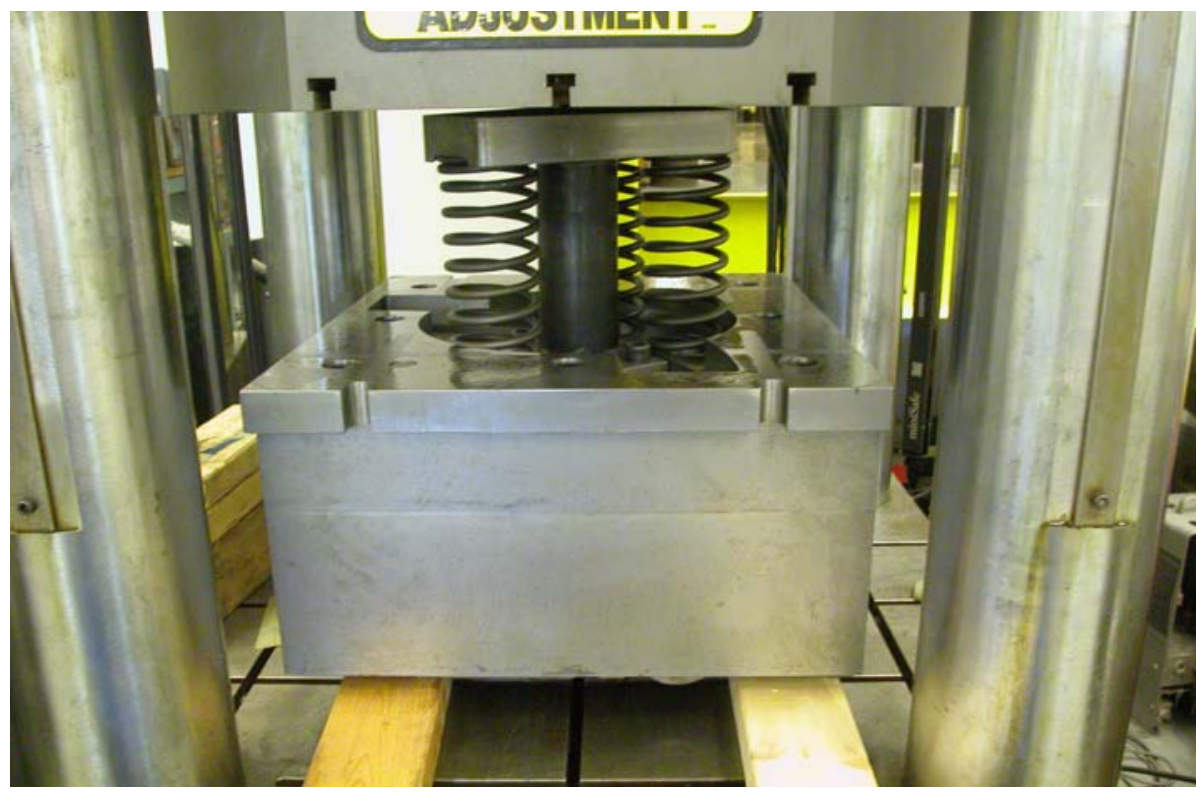

Figure 5.6 Photo of punch and clamp holder in hydraulic press 


\subsubsection{Electromagnetic coil design}

As mentioned in Section 5.2.1, electromagnetic coils should be embedded into the top surface of the die to encourage the metal draw-in from the flange region into die cavity. To encourage the draw-in, electromagnetic forces should be applied on the flange region. The basic rules of electromagnetic coil design are as followings

(1) The coil position should be close to the die profile;

(2) There should be some space left between the coil and the die profile, where the blank holder can press the blank down to prevent wrinkling;

(3) There must be return path for the induced current in the metal blank. Otherwise, the electromagnetic forming has low efficiency. So the electromagnetic coil was designed to have 2 leads in both inner and outer sides;

(4) There must be a hollow groove in the blank holder to provide forming space for electromagnetic forming;

According to above rules, electromagnetic coil was designed and made of Cooper 110 by water jet, shown as Figure 5.7. To prevent the repulsive electromagnetic force between the two leads, the gap between them was deigned to be twice of the width of the electromagnetic coil. The width of the electromagnetic coil was determined as 0.125 inch. To insulate the current from the steel tooling and the press, a G10 lower plate and a G10 clamp plate were designed, shown as Figure 5.8 and 5.9. Electromagnetic coil was embedded in the G10 lower plate. And there was a hollow groove just above the electromagnetic coil in the G10 clamp plate to provide forming space. The hollow groove was determined to have 0.5 inch width and two 0.25 -inch-radius edges. 


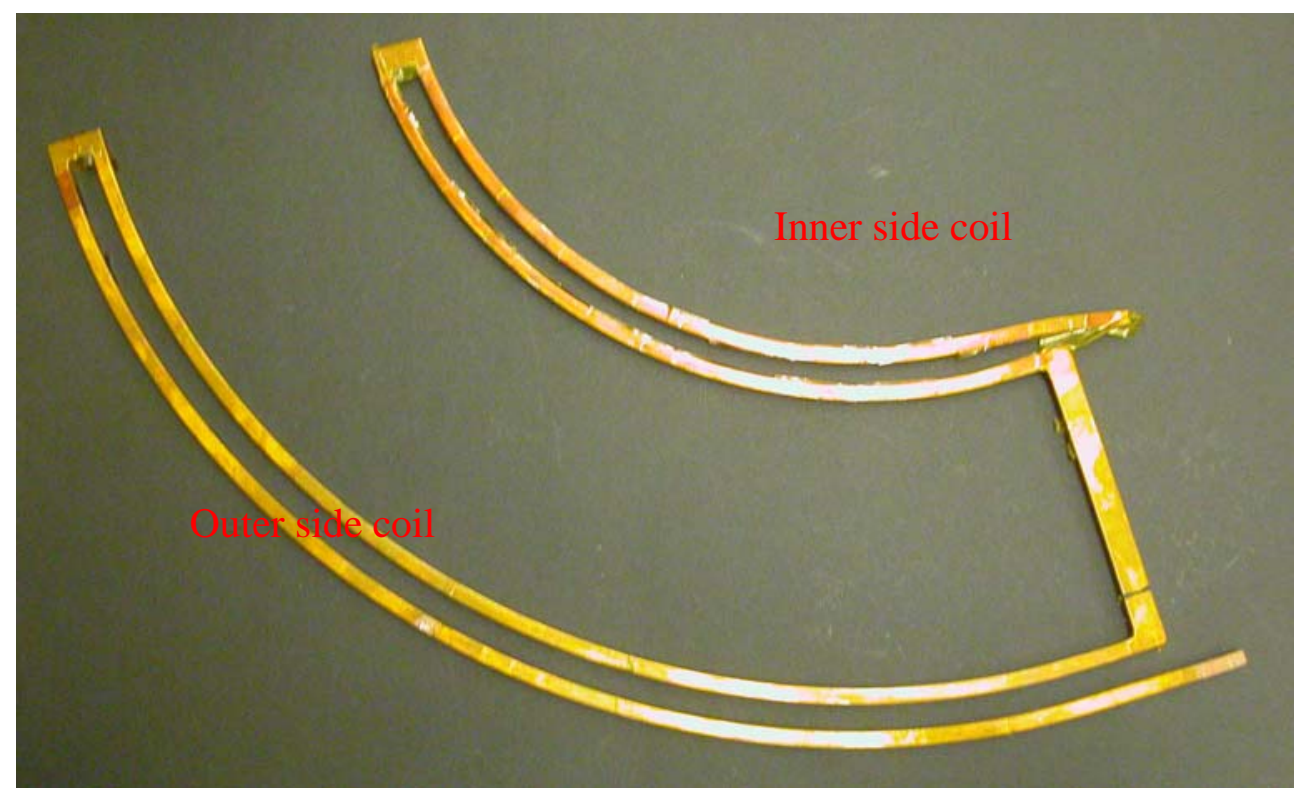

Figure 5.7 Electromagnetic coil (Made of Cooper 110) insulated by Kapton tape

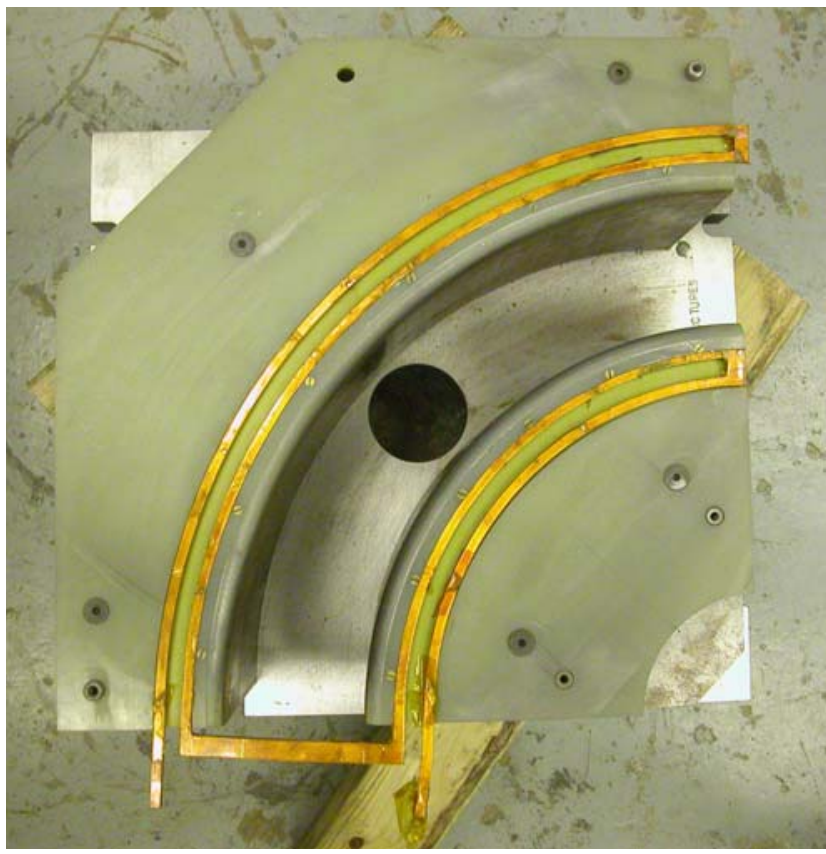

Figure 5.8 Electromagnetic coil and the die 


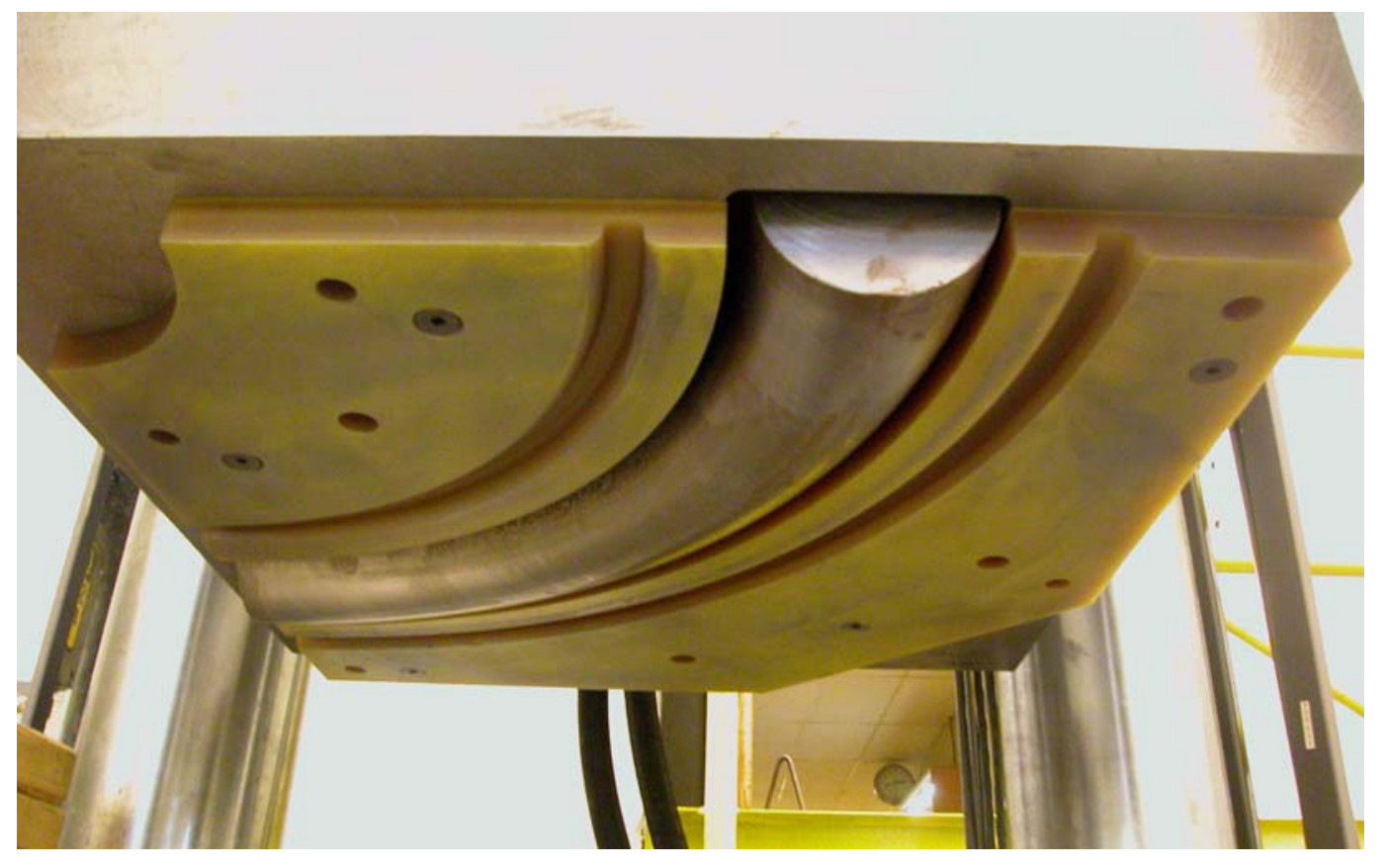

Figure 5.9 G10 plate, punch and upper clamp holder in hydraulic press

\subsubsection{Blank material and size design}

As mentioned before, the original part was made from 2219-O with 0.09inch thickness. The tooling was also scaled down to one third of the original tooling. So the experiment blank was also scaled down to one third of the original blank, i.e. 0.032inch thick. And the material of blank was also 2219-O.

Blank size is important for a successful stamping. For this case, an annular-arc-shape blank was developed at first, shown as Figure 5.10. This blank has 16-inch-diameter outer side and 6-inch-diameter inner side. It was assumed that the flanges of this kind of blank would move into the die cavity along the radial direction. While, the experiment 
results turned out that this blank shape was unsuitable. In fact, the metal of the flange moved into die cavity along the direction parallel to the center line of tooling, instead of the radial direction. So it is easy to move out of clamp holder. Moreover, the inner side of the blank was too short.

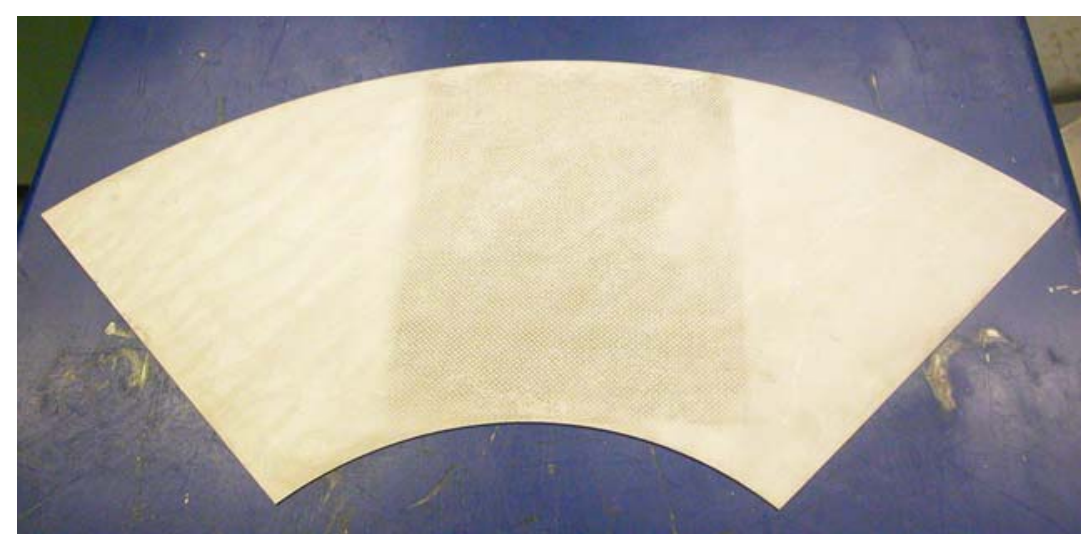

Figure 5.10 Photo of annular-arc-shape blank

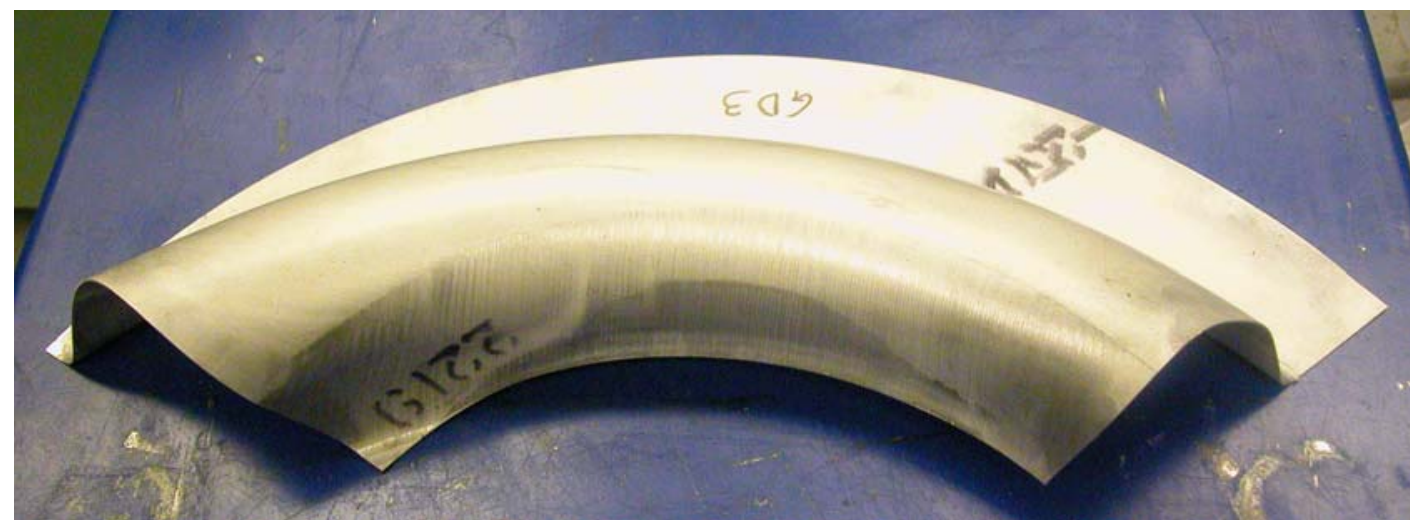

Figure 5.11 Photo of part after stamping with annular-arc-shape blank 
After several trials, the blank size was determined as a polygon with 18-inch length and 16-inch width, shown as below. This kind of blank could make sure that the inner side has enough material and the outer side will not move out of clamp holder.

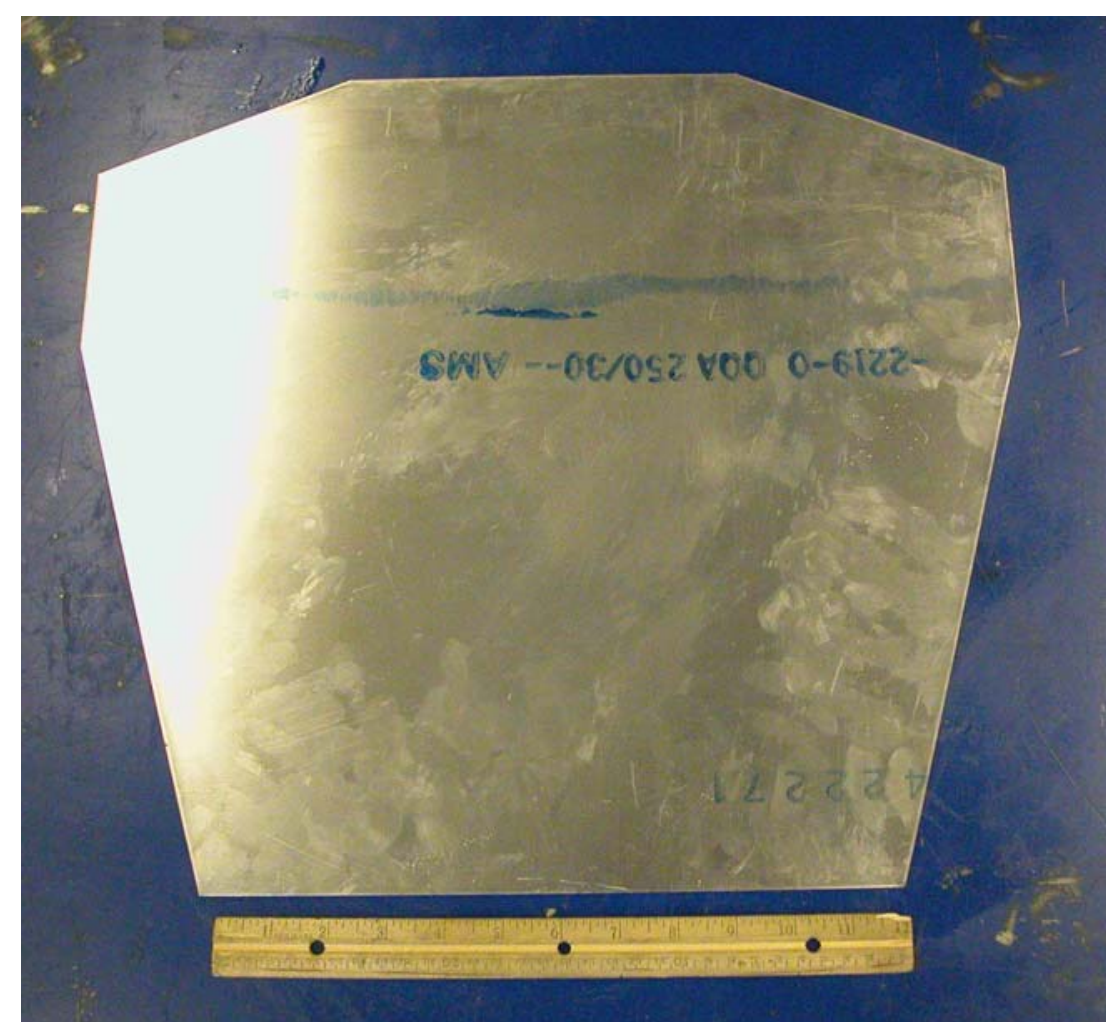

Figure 5.12 Polygon blank with 18-inch length and 16-inch width

\subsubsection{Experiment procedure}

For conventional stamping process, the experiment procedure is simple. The blank holder clamps the blank, and the punch advances until the failure of the blank. 
For electromagnetically assisted sheet metal forming, the experiment procedure is relative complex, shown as below.

(1) The blank holder clamps the blank;

(2) The punch advances and stops at a pre-decided position;

(3) A electromagnetic pulse with predefined energy level is given to the coil and deforms the blank to make a stretched "bubble" at the flange area;

(4) Repeat (2) and (3) until the failure of the blank;

The punch advance should be coupled with the EM energy level. EM energy pulse will stretch the "bubble" ring and create extra line length, which can be pulled and converted to the wall region by the following punch advance. And EM energy should be suitable. Large EM energy will stretch too much the "bubble” ring and weaken the metal sheet. Moreover, there are repulsive EM forces between the two leads of the EM coil for this specific case. So to apply EM energy, it should be considered to avoid large repulsive EM forces. All of these can be tried by trial-and-error or finite element analysis.

To determine the EM energy level used, different EM energies were applied to the blank. After several trials, $2.4 \mathrm{~kJ}$ per pulse was determined to apply for EM deformation. The length of "bubble" ring was measured about 1.025 inches, which meant there was 0.125 inch extra line length created. Therefore, the punch advance was set as 0.125 inch after every EM pulse. While, the punch advance was set as 0.25 inch after every EM pulse from the beginning of forming to around 1 inch punch stroke, because there would be plastic stretching at the wall of metal part. 


\subsection{Experiment results}

\subsubsection{Stamping with conventional stamping and electromagnetic forming}

First, the parts were produced by conventional stamping by several forming conditions.

With 10 klbs clamp load, the part appeared wrinkling on the inner side, as shown in

Figure 5.13. While with $30 \mathrm{klbs}$ clamp load, the part had early tearing on the outer side, as shown in Figure 5.14.

\begin{tabular}{ccccc}
\hline No. & Clamp load & EM pulse & Draw depth & Comment \\
\hline $1 \#$ & $10 k l b s$ & No & $2.580 ”$ & Serious wrinkles \\
\hline $2 \#$ & $10 k l b s$ & 21 & $2.189 ”$ & Wrinkles appeared \\
\hline $3 \#$ & $30 k l b s$ & No & $1.563 ”$ & Tearing at the outer side \\
\hline $4 \#$ & $30 k l b s$ & 17 & $2.482 ”$ & Wrinkles appeared, only used outer coil \\
\hline
\end{tabular}

Table 5.1 Results of forming 0.032-inch-thick 2219-O

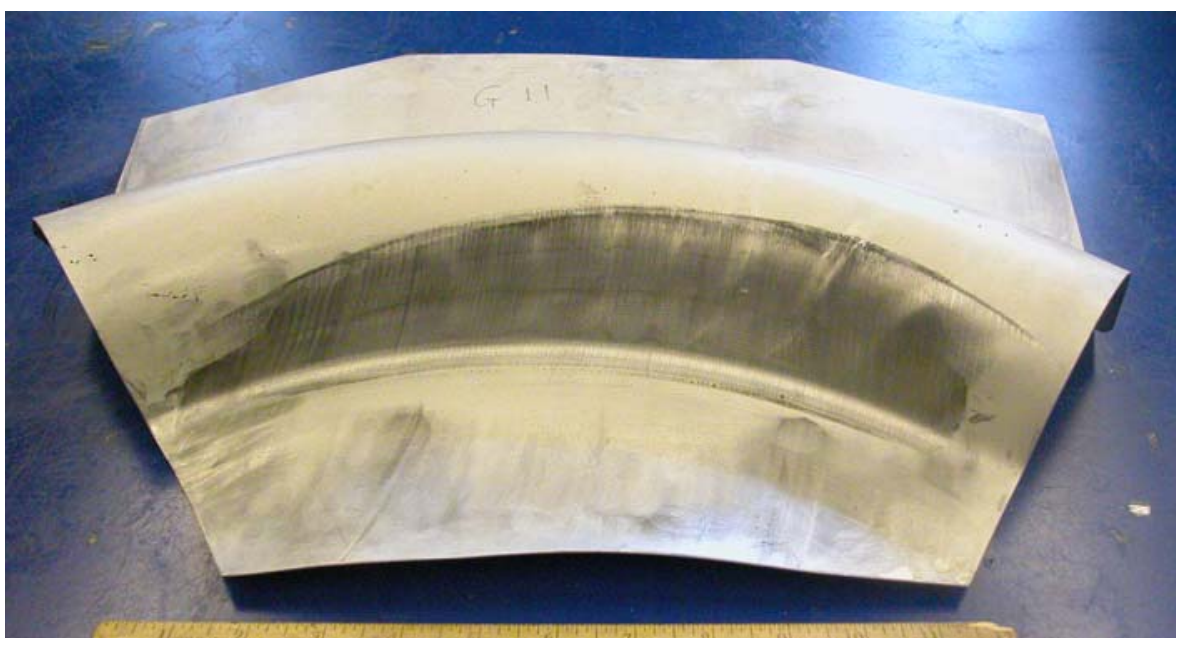

Figure 5.13 Photo of sample 1\# (Draw depth: 2.580 inches, serious wrinkling on inner) 


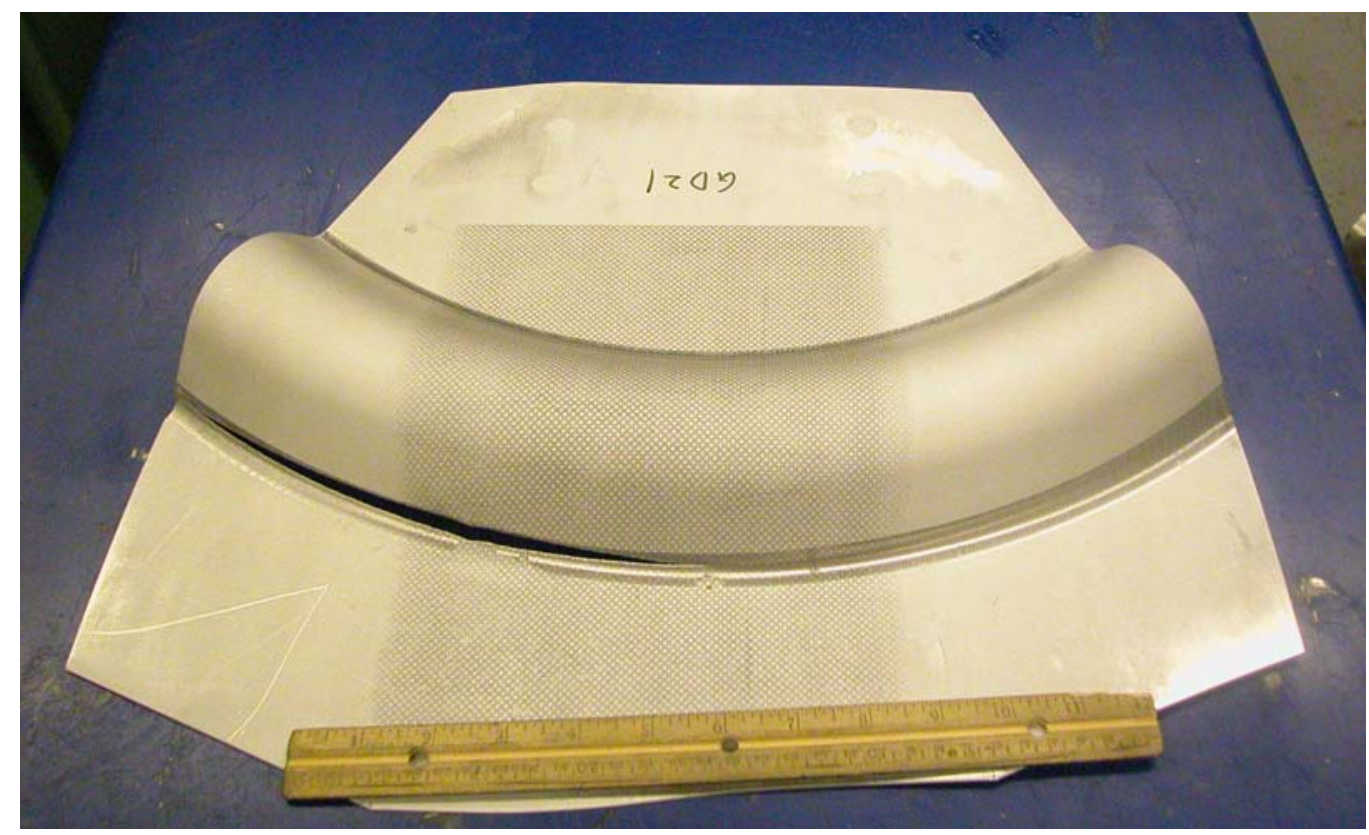

Figure 5.14 Photo of sample 3\# (draw depth: 1.563 inches, tearing on outer side)

Above two experiment results show that the metals of inner side flange are easy to draw into the die cavity because of the larger size of outer side flange. So it has the tendency to wrinkle at the inner side wall. To prevent the wrinkling, i.e. to prevent the drawing-in from the inner side flange, higher clamp load should be applied, which will in turn cause tearing at the outer side wall.

To encourage the metal to flow into the die cavity from the flanges, electromagnetic forming was applied. As mentioned before, $2.4 \mathrm{~kJ}$ per pulse was determined to apply for EM deformation. Both the inner side coil and the outer side coil were used to provide electromagnetic forces to the inner and outer side flange. Figure 5.15 is the photo of the 
part produced by $21 \mathrm{EM}$ pulses at $10 \mathrm{klbs}$ clamp load. After $21 \mathrm{EM}$ pulses, the draw depth was increased to 2.189 inches. And the wrinkling appeared on the inner side wall.

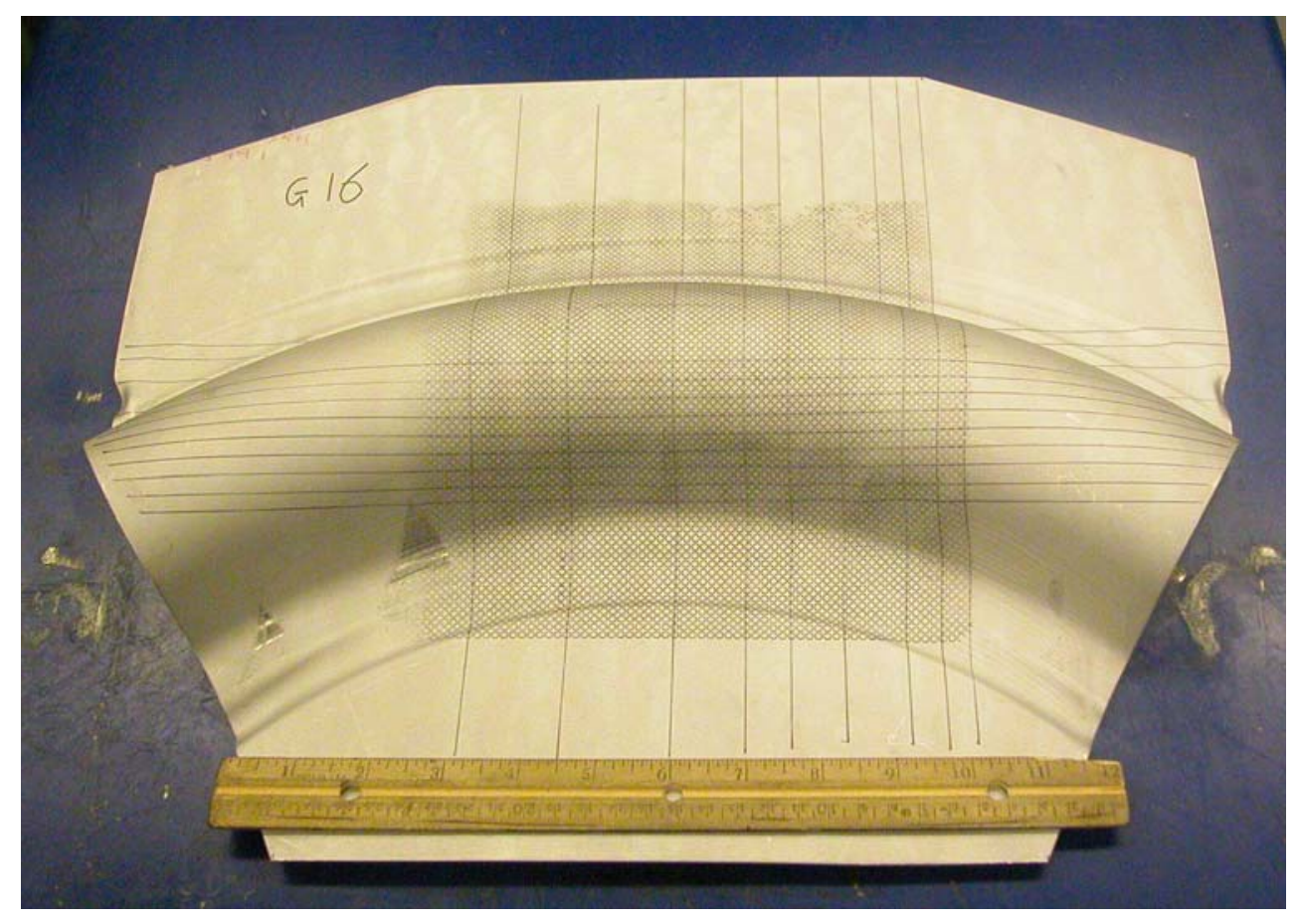

Figure 5.15 Photo of sample 2\# (Clamp load: 10 klbs; Draw depth: 2.189”; 21 EM pulses with $2.4 \mathrm{~kJ}$ per pulse)

Above experiment shows that EM forces had littlie effect on the improvement of draw depth at low clamp load condition. EM forces are applied to encourage the drawing-in of metal from the flange into die cavity, and there is tendency to wrinkle at low clamp load condition at the inner side wall. To get ride of wrinkling, the metal flow should be restricted from the inner side flange into die cavity. EM forces at the inner side actually 
encouraged the draw-in from the flange region, which led to the wrinkling of the inner side wall. Therefore, the inner side coil is not helpful since the inner side materials are easier to flow into the die cavity.

To get ride of wrinkling, higher clamp load (30 klbs) was applied and only outer side coil was used. Since there was only outer side coil, the EM energy delivered per pulse decreased to $1.2 \mathrm{~kJ}$. Figure 5.16 and 5.17 are the photos of part made by $17 \mathrm{EM}$ pulses at 30 klbs clamp load. After 17 EM shots, the draw depth was increased to 2.482 inches. And the wrinkling appeared on the inner side wall.

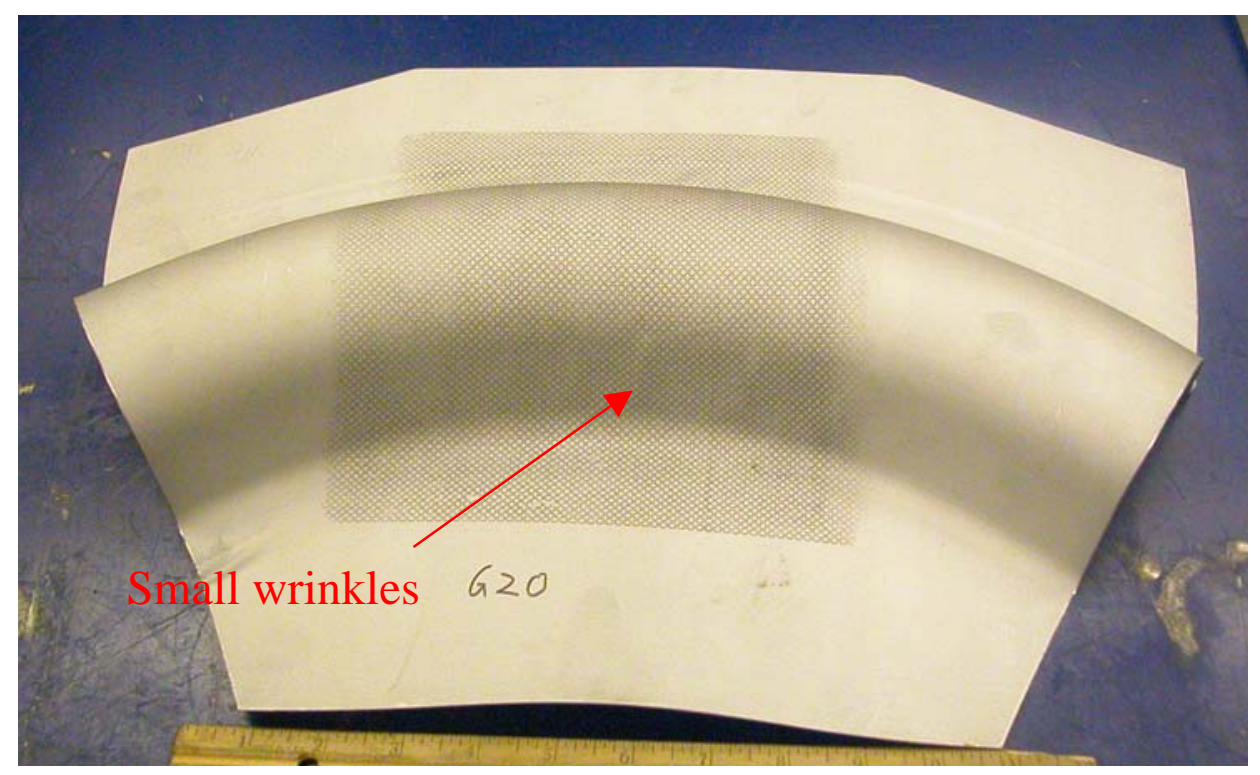

Figure 5.16 Inner side of Sample 4\# (Clamp load: 30 klbs; Draw depth: 2.482”; 17 pulses with $1.2 \mathrm{~kJ}$ per pulse) 


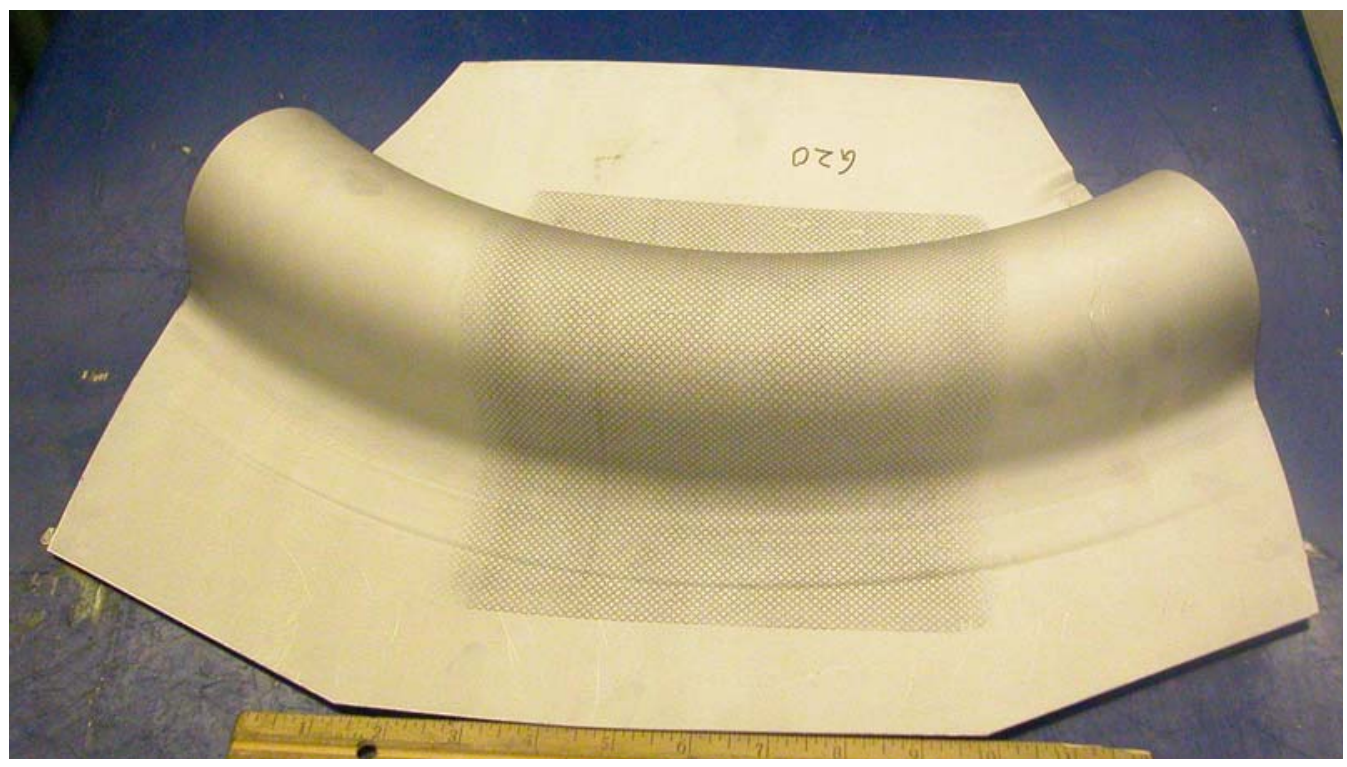

Figure 5.17 Outer side of Sample 4\# (Clamp load: 30 klbs; Draw depth: 2.482”, 17 EM pulses with $1.2 \mathrm{~kJ}$ per pulse)

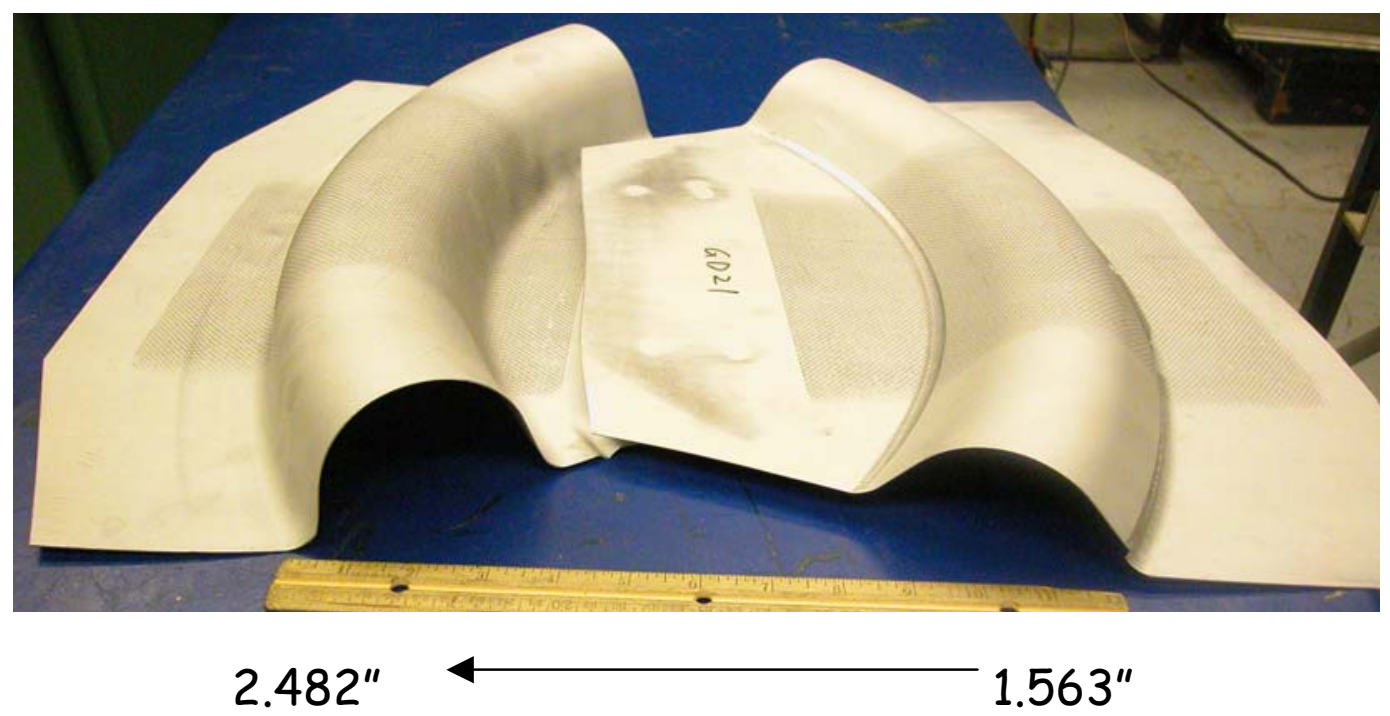

Figure 5.18 Comparisons of Sample 3\# and 4\# 136 
Comparing sample 3\# and 4\#, shown as Figure 5.18, the draw depth was increased by $59 \%$ because of the EM pulses at the outer side flange. The outer side EM pulses improved the metal flow from the outer side into die cavity, and reduced the tension at the outer side wall to avoid the tearing, since more materials could be easier to draw into the die. Therefore, larger draw depth was achieved.

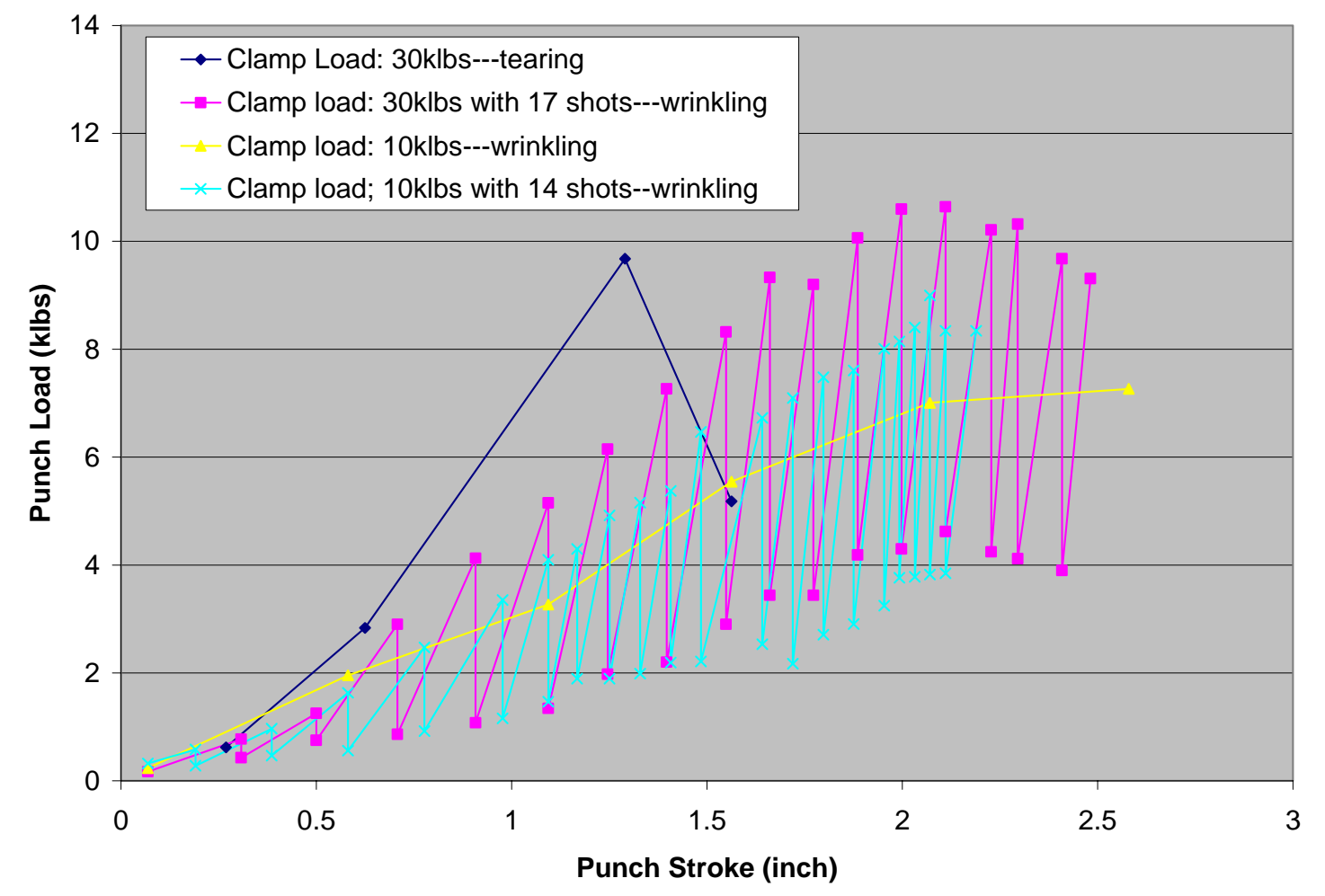

Figure 5.19 Punch load and punch stroke of Sample 1\# 4\#

Above is the diagram of the punch load and the punch stroke for sample 1\#, 2\# 3\# and 4\#. It clearly shows that the punch load dropped after every EM pulse and then increased 
with the punch advance. For low clamp load, the EM pulses had littlie effect on draw depth because of wrinkling at the inner side wall. And for high clamp load, the EM pulses had large effect on draw depth because the metals were easier to flow from outer side flange into die cavity.

Therefore, for this experiment, the wrinkling at the inner side wall becomes the most important issue. It causes the failure of the part. Although the experiments showed that EM pulses could improve the drawing-in from the flange, the drawing-in from the inner side flange should be restricted to avoid wrinkling.

To control the metal flow from the flange, one possible way is to vary the clamp load with the punch advance. This method can be effective but will increase the complexity of the experiment. To find an optimal procedure of varying clamp load needs lots of experiments. So to show the feasibility of EM forming in relative simple way, the clamp load was kept constant during the forming process.

\subsubsection{Stamping with shimming at inner side}

Above experiments show that the wrinkling at the inner side wall caused failure of parts. To avoid wrinkling, the metal flow from the inner side flange should be restricted. There are two ways, increasing the clamp load or shimming at inner side. Increasing the clamp load will also affect the metal flow from the outer side flange which should be encouraged. So shimming was applied at the inner side flange to restrict the metal flow. 
For the purpose of shimming, a piece of paper was put at the inner side of clamp surface to provide higher pressure at inner side flange, shown in Figure 5.20.

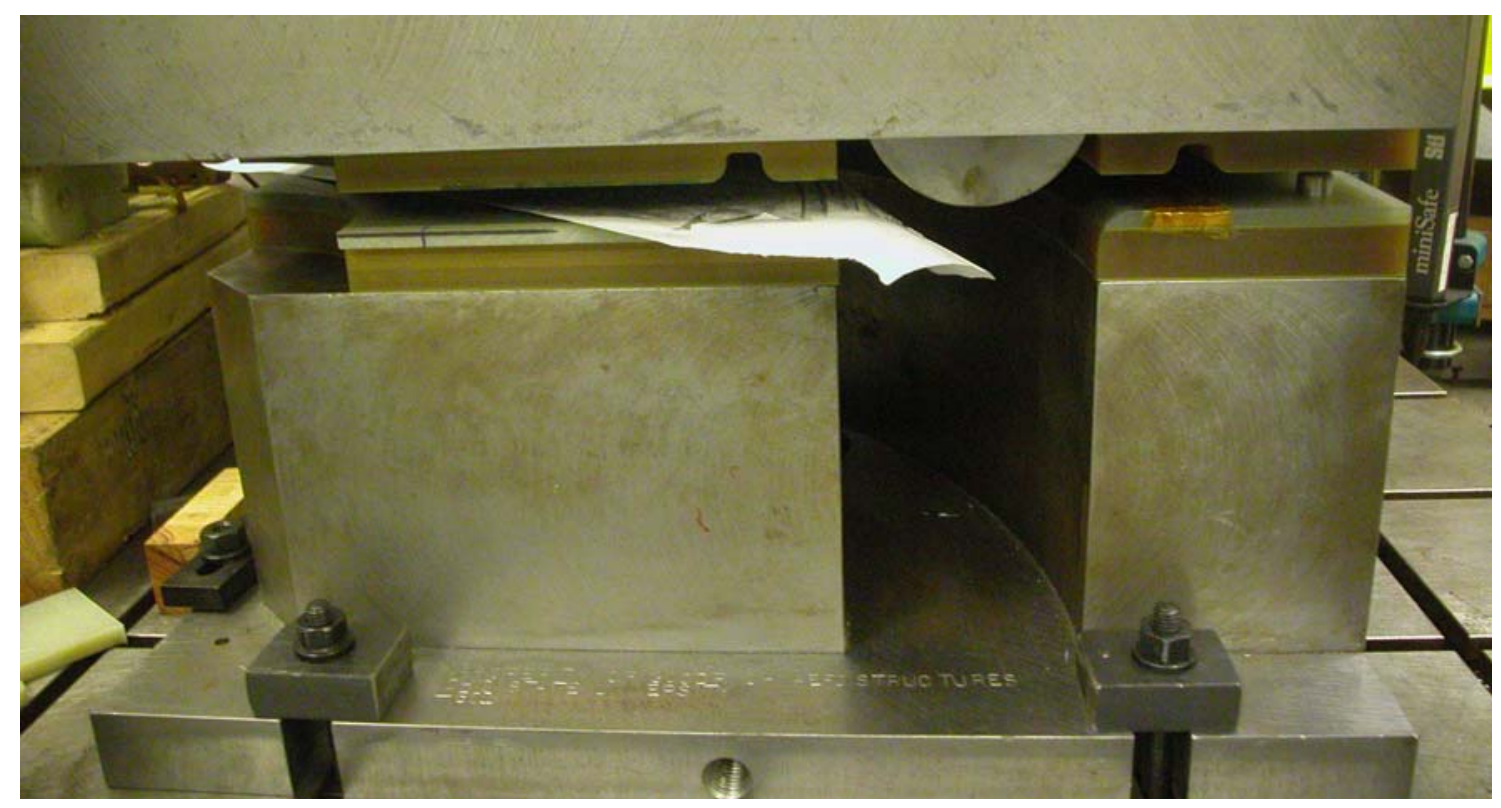

Figure 5.20 Paper for shimming at the inner side clamp

And to increase the efficiency of the coil, a new 3-bar coil was used at outer side clamp area, shown in Figure 5.21. The two-lead coil used before has less efficiency than the 3bar coil. The middle bar of the 3-bar coil has twice current density than the other two bars. Therefore the middle position of the metal inside of the hollow groove will get the higher repulsive EM force. The width of the 3-bar coil is 0.1 inch and the gap between two bars is also 0.1 inch. 


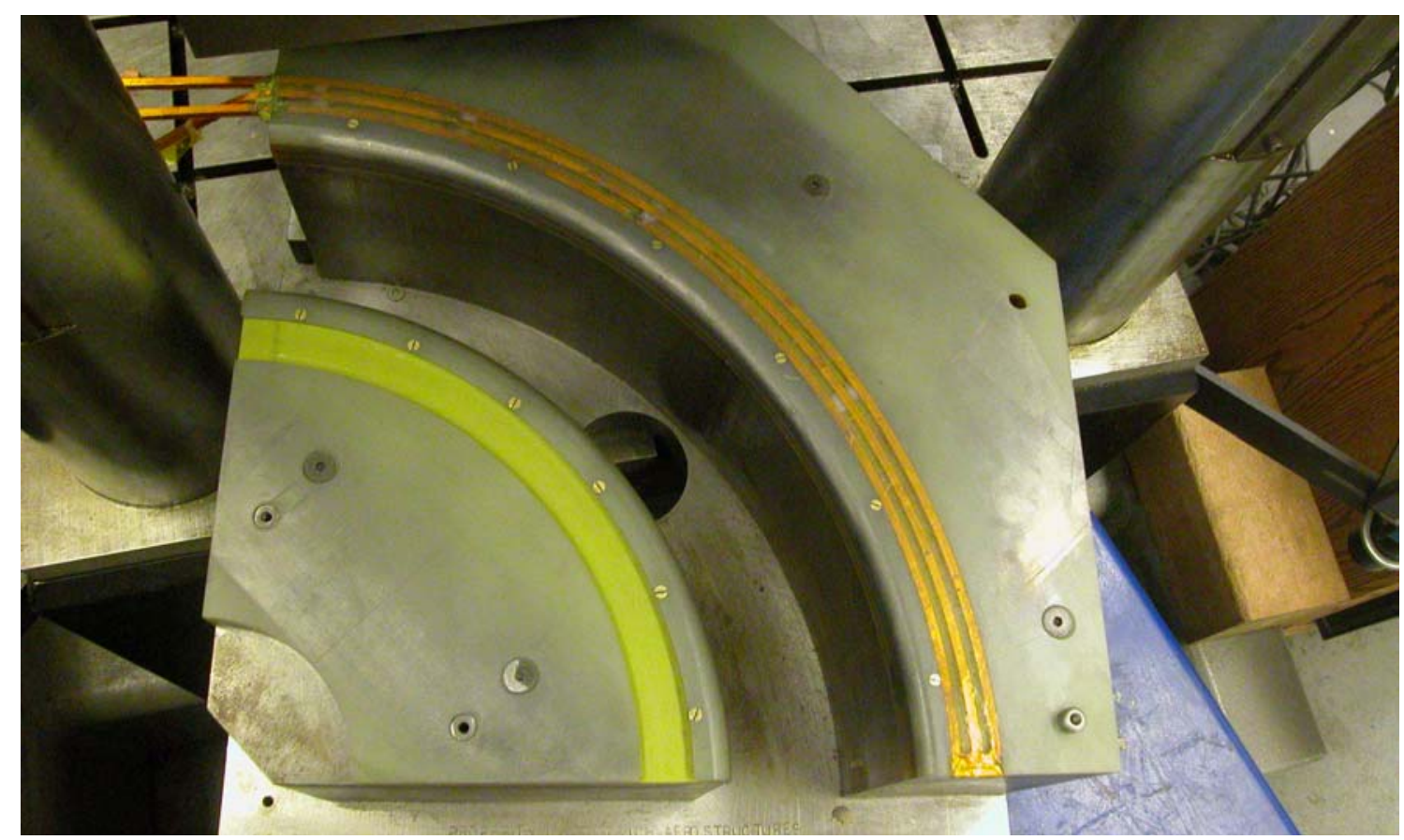

Figure 5.21 Photo of 3-bar coil at outer side die

0.032-inch-thick 2219-O was used in these experiments. While these blanks were cut out from different batches from the blanks used before. They showed a higher strength than those in last experiments (section 5.3.1). Figure 5.22 is the photo of part made of new 2219-O blank. This blank was formed by conventional forming with 30-klb clamp load and appeared wrinkling at the inner side wall instead of tearing. In previous experiment, the blank had tearing at the outer side wall with 30-klb clamp load, shown as Figure 5.14. Below are the summary of the experiment results with shimming at the inner side clamp. 


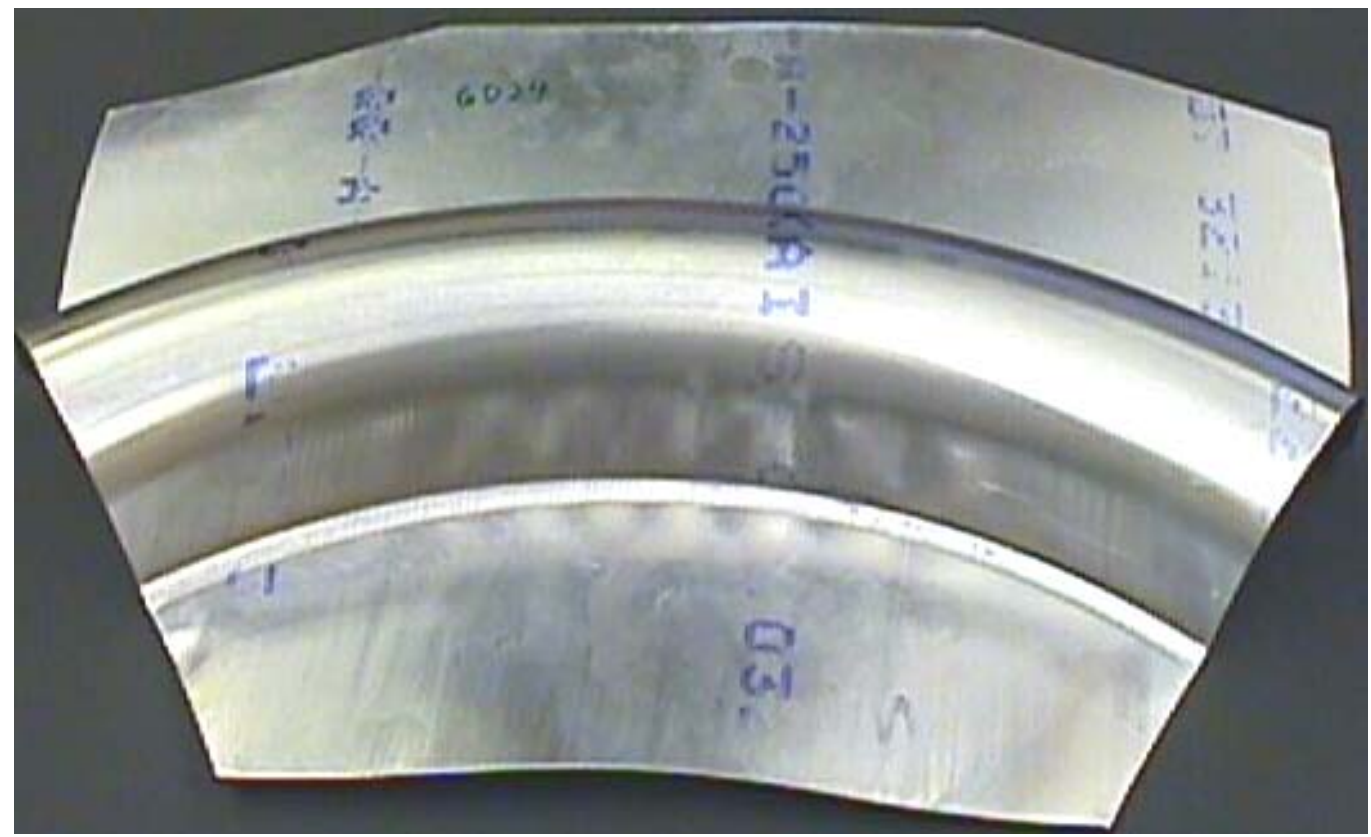

Figure 5.22 New 0.032-inch-thick 2219-O (Clamp load: 30 klbs; Draw depth: 2.633”; wrinkling appeared at the inner side wall) 


\begin{tabular}{|c|c|c|c|c|c|}
\hline No. & $\begin{array}{l}\text { Clamp } \\
\text { load }\end{array}$ & EM shot & Coil & $\begin{array}{l}\text { Draw } \\
\text { Depth }\end{array}$ & Comment \\
\hline $5 \#$ & 30klbs & No & & $2.633 ”$ & $\begin{array}{l}\text { Wrinkles at inner side; without } \\
\text { shimming }\end{array}$ \\
\hline $6 \#$ & 30klbs & No & & 1.930” & Tearing at inner side \\
\hline 7\# & $20 \mathrm{klbs}$ & No & & $4.603 ”$ & Serious wrinkles at inner side \\
\hline 8\# & 25klbs & No & & $3.268 ”$ & Wrinkles appeared at inner side \\
\hline 9\# & 30klbs & $\begin{array}{l}120 \text { shots } \\
1.2 \mathrm{~kJ} / \text { shot }\end{array}$ & Outer & $3.376 ”$ & Wrinkles appeared at inner side \\
\hline $10 \#$ & 30klbs & $\begin{array}{l}50 \text { shots } \\
2.4 \mathrm{~kJ} / \text { shot }\end{array}$ & Outer & $3.376 ”$ & Wrinkles appeared at inner side \\
\hline $11 \#$ & 40klbs & $\begin{array}{l}21 \text { shots } \\
2.4 \mathrm{KJ} / \text { shot }\end{array}$ & Outer & $1.563 ”$ & Tearing at inner side \\
\hline 12\# & 35klbs & $\begin{array}{l}29 \text { shots } \\
2.4 \mathrm{KJ} / \text { shot }\end{array}$ & Outer & $1.798 ”$ & Tearing at inner side \\
\hline 13\# & 30klbs & $\begin{array}{l}21 \text { shots } \\
2.4 \mathrm{KJ} / \text { shot }\end{array}$ & Inner & $2.042 ”$ & Tearing at inner side \\
\hline
\end{tabular}

Table 5.2 Forming 0.032” 2219-O with shimming at inner side flange

\subsubsection{Effect of shimming}

Comparing Sample 5\# and 6\#, with same clamp load, Sample 6\# has no wrinkling in the inner side wall and has tearing in the outer side wall instead, shown as Figure 5.23. It shows the effect of shimming. Because of the shimming at the inner side clamp, the metal is restricted to flow from the inner side flange into the die cavity, which avoids the wrinkling in the inner side. But to form the part, more metals need to draw from the flange into die cavity. Larger tension force is needed at the inner side wall, since the inner side flange has higher pressure. The larger tension force causes tearing at the inner side wall. 


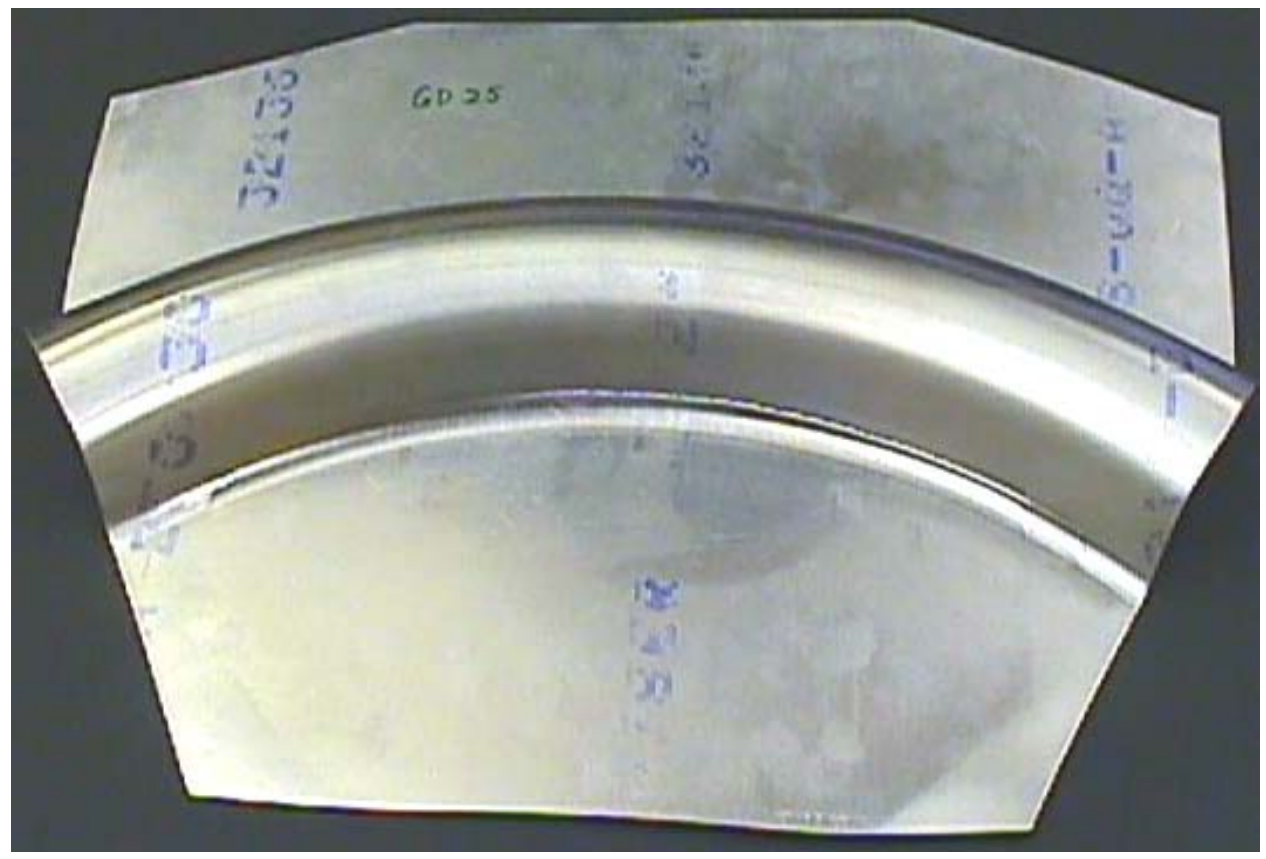

Figure 5.23 Sample 6\# (Clamp load: 30 klbs; Draw depth: 1.930”; Tearing at the inner side wall; with shimming at the inner side flange)

Sample 6\#, 7\# and 8\# show the effect on clamp load with shimming at the inner side. It shows that the clamp load lower than 30 klbs produced wrinkling at the inner side. To avoid wrinkling, higher clamp load should be applied, which will in turn cause early tearing. Therefore, 30-klb clamp load was taken as the base line to apply EM force.

\subsubsection{Effect of EM forming with shimming}

The shimming at the inner side flange restricts the metal flow from the inner side, and in turn makes the metal flow easier from the outer side flange. Since EM forming also is applied to encourage the metal flow from the outer side flange into die cavity, will EM 
forming still improve the metal flow? The experiment results show that it did improve the metal flow from the outer side flange. Figure 5.24 is the photo of part made by 120 EM pulses. The draw depth was increased to 3.376 inches. And there was wrinkles appearing at the inner side wall.

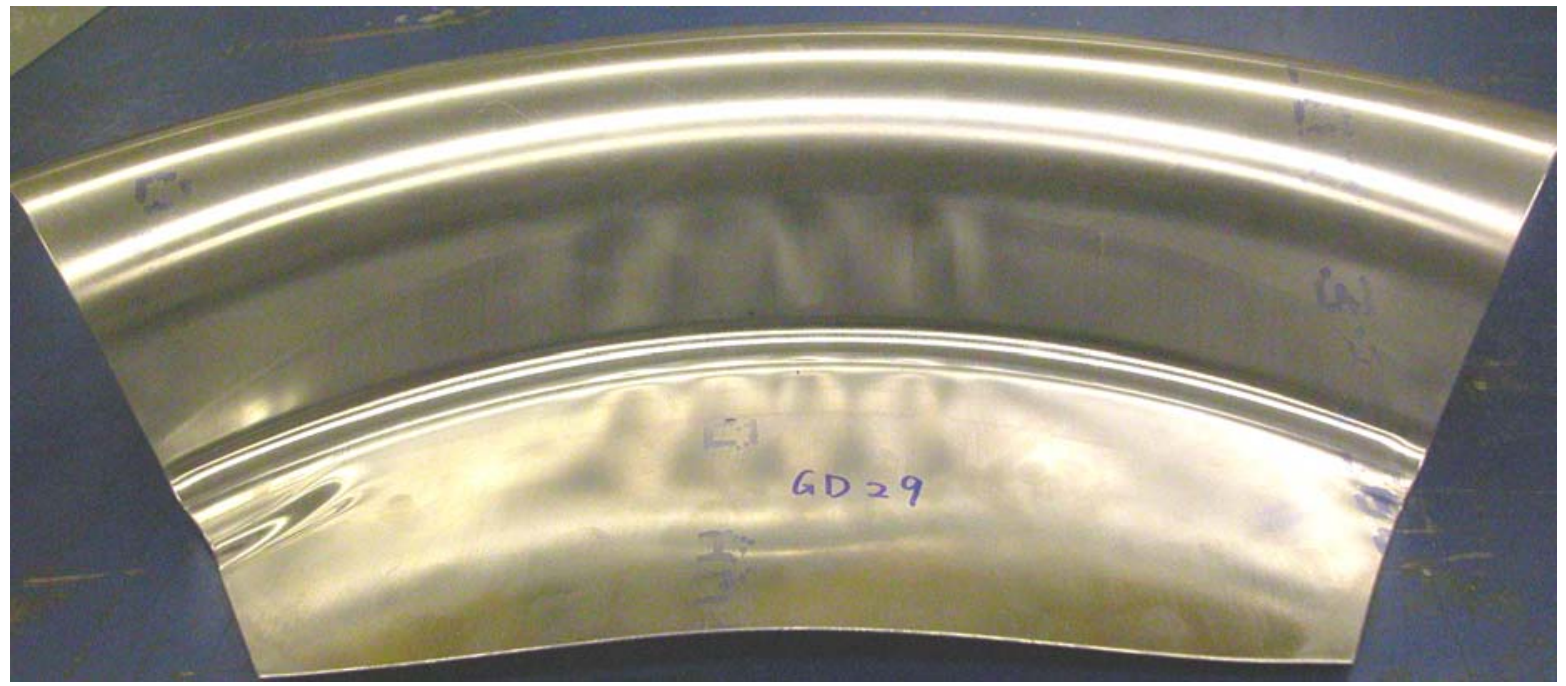

Figure 5.24 Photo of sample 9\# (Clamp load: 30 klbs; Draw depth: 3.376 inches; 120 EM pulses with $1.2 \mathrm{~kJ}$ per pulse; Wrinkling at the inner side wall; shimming at the inner side)

From sample $6 \#$ to sample $9 \#$, the draw depth increased by $75 \%$. The improvement should be caused by EM forces. Since shimming causes larger stretching forces for inner side, the tearing happened at the inner side wall without EM forces. With EM forces, the outer side flange will have some "bubble" ring. When punch advances, this "bubble" ring will be pulled out first. During this pulling process, only partial outer side flange will 
involve the deformation, instead of the whole outer side flange. Therefore, the stretching force at the outer side wall will be smaller, which will cause the loose at the inner side wall and reduce the tension force at the inner side wall.

\subsubsection{Effect of EM energy level with shimming}

Two kinds of energy levels were applied to examine the effect of EM energy level. The first one was $1.2 \mathrm{~kJ}$ per pulse. The second one was $2.4 \mathrm{~kJ}$ per pulse. The results show that the EM energy level per pulse had no effect on the draw depth. The same draw depths were achieved with these two energy levels. Higher energy level needed less EM pulses and larger punch advance between the EM pulses, because higher energy stretches more "bubble” ring. For the $2.4 \mathrm{~kJ}$ per pulse case, there were $50 \mathrm{EM}$ pulses fired. For the $1.2 \mathrm{~kJ}$ per pulse case, there were $120 \mathrm{EM}$ pulses fired.

EM energy level per pulse should be carefully determined. The purpose of EM forming at the flange is to stretch the flange, to draw the outer flange into the hollow groove and to make a partial flange drawing. Small energy level does not provide enough stretching of the flange, and then can not draw much metal from the outer side flange rim into the hollow groove. Too high energy level will stretch too much and weaken the metal sheet, which will cause tearing later. 


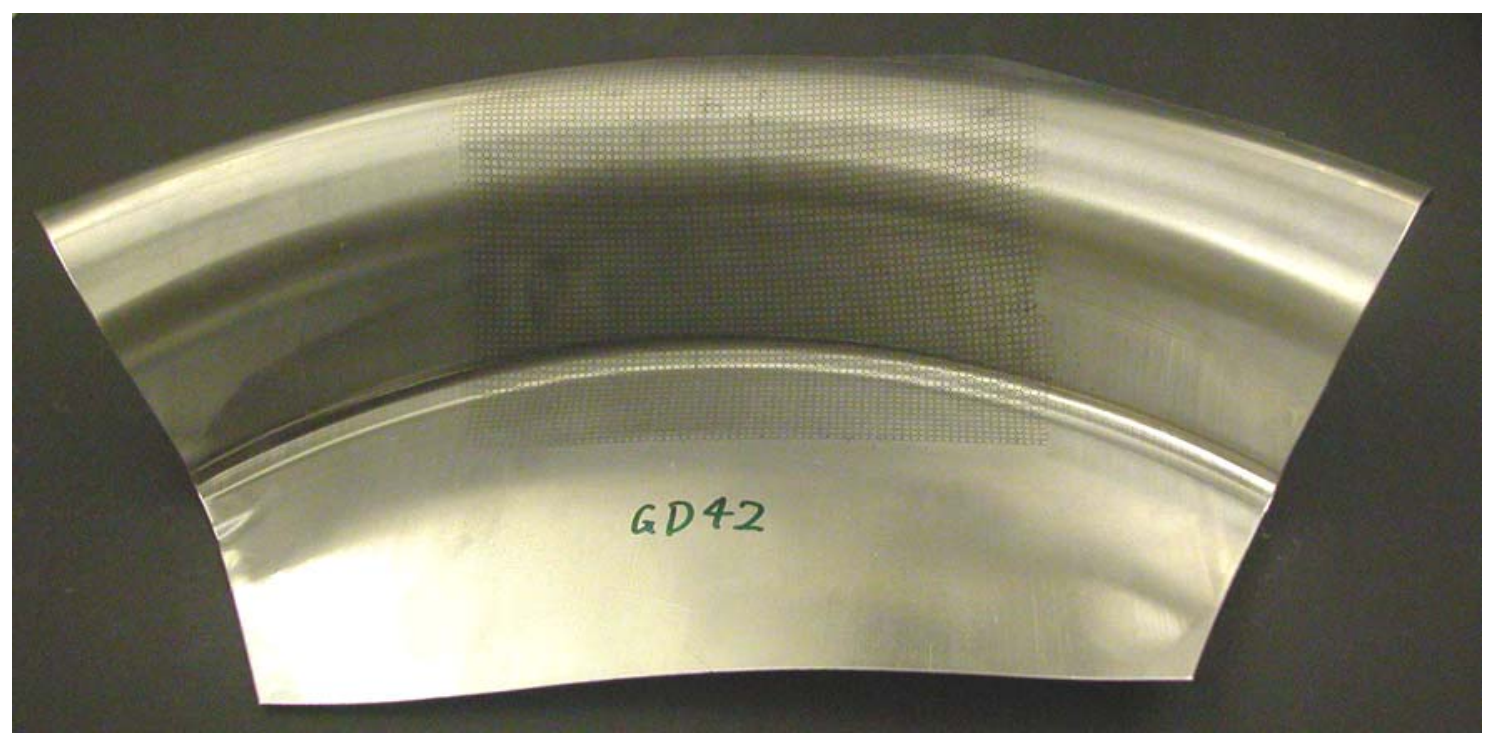

Figure 5.25 Photo of sample 10\# (Clamp load: 30 klbs; Draw depth: 3.376 inches; 50 EM shots with $2.4 \mathrm{~kJ}$ per shot; Wrinkling at the inner side wall; shimming at the inner side)

\subsubsection{Effect of clamp load for EM assisted stamping with shimming}

Clamp load has large effect on the draw depth. Large clamp load will make the drawingin difficult from the flange. Three different clamp loads, 30 klbs, 35 klbs and 40 klbs, were applied to research the effect of clamp load, as Sample 10\#, 11\# and 12\#. The results show that this lip-skin panel process is sensitive to the clamp load. The larger clamp load caused the early tearing at the inner side wall. To avoid the tearing at the inner side, 30 klbs clamp load was suitable. 


\subsubsection{Effect of EM coil at the inner side}

As said before, inner side wall has the tendency to wrinkling. So to restrict the metal flow into the inner side wall, the shimming at the inner side was applied, which caused tearing at the inner side wall when clamp load was high. Excessive tension causes tearing. If the metal is easier to flow from the inner side flange into die cavity, the tension will be reduced. Therefore, to reduce the tension at the inner side wall, is it useful to apply EM coil at the inner side meanwhile with shimming? The experiment result showed that this method was not positive.

Figure 5.26 is the photo of the part with both inner side EM coil and the shimming. It shows there was tearing at the inner side flange just above the EM coil. And the draw depth was 2.042 inches. The inner side EM coil did not improve the draw depth.

It is reasonable. Because of the shimming at inner side, the inner side has higher pressure. To draw metal from the inner side flange needs larger stretching force. If using inner side

coil, the stretching caused by EM forces will thin the blank, which actually will lead to tearing. While, if the pressure at the inner side flange is not high enough, the inner side coil improves the metal flow to die cavity and more materials at inner side wall will cause wrinkles. Therefore, the coil at the inner side actually has no positive effect. 


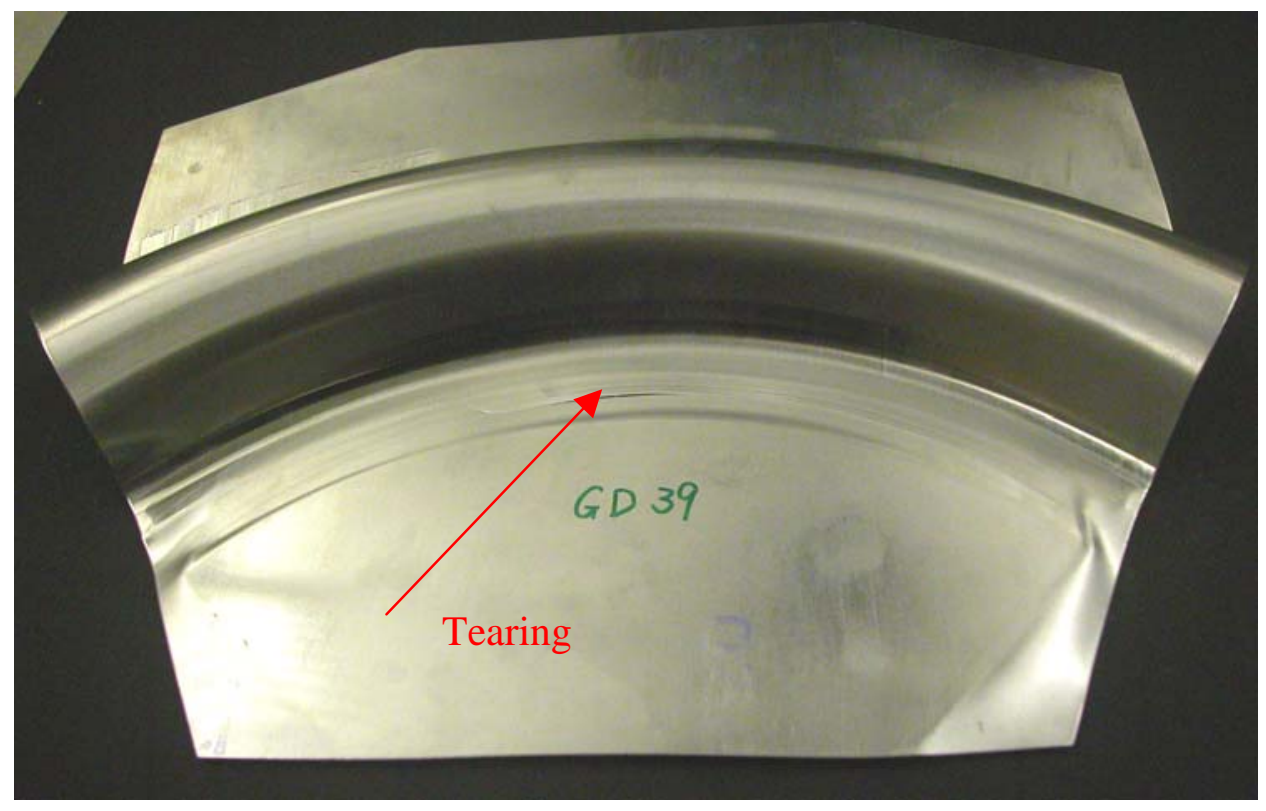

Figure 5.26 Photo of sample 13\# (Clamp load: 30 klbs; Draw depth: 2.042”; 21 pulses with $2.4 \mathrm{~kJ}$ per pulse; inner side coil; shimming at inner side flange)

\subsection{Conclusion}

To improve the draw depth of lip-skin panel, electromagnetic coils were applied at the die to encourage the metal flow from the flange into die cavity. This experiment shows that both proper control of blank holder force and electromagnetic forces at the process have an important influence on the forming process. And to reach the maximal draw depth, a suitable sequence of varying blank holder force with punch stroke is needed to investigate. This work also demonstrates the subtle tuning of the forming parameters is required to produce the lip-skin panel. The lip-skin forming process is very sensitive to the forming parameters. 
For this particular lip-skin panel, the best draw depth was obtained with a hybrid electromagnetic assisted approach. But it was only modestly better than the one with optimized blank holder forces. For this lip-skin panel, the EM pulses have a similar effect to reducing blank holder forces, i.e. to encourage draw in. The effect of EM pulses can be done by careful controlling the blank holder forces, especially the blank holder forces for the outer side flange. For this particular case, there is no persuasive benefit to apply electromagnetic pulses. 


\section{CHAPTER 6}

\section{APPLICATION OF ELECTROMAGNETIC FORMING AT BOTTOM OF METAL PART}

There are two possible regions to apply electromagnetic forming in a stamping process, the flange of metal part and the bottom of the metal part. The last two chapters discussed the application of electromagnetic forming at the flange of metal part. This chapter will investigate the application of electromagnetic forming at the bottom of metal part.

The bottom of metal part is below the punch face. The punch advances to draw the metals from the flange into die cavity. The blank holder clamps the flange so that there is tension to stretch the sheet over the punch. So the bottom region is in bi-axial stretching state. Because of the friction between the punch and the bottom of the metal part, this region has small stretching in conventional stamping (usually 3\% 8\% depending on the radius of punch profile, clamp load, lubrication, etc) [1, 88]. Therefore, most of deformation happens at the flange and the side wall. The failures usually are the tearing near the punch nose, or the wrinkling at the flange.

Since the bottom has small deformation, it is possible to increase the part depth by increasing the strain in the bottom. Increasing clamp load improves the deformation of 
the bottom region, but also makes it difficult to draw the flange into die cavity. Lubrication does increase the stretching of the bottom region, while the improvement is not much. The large radius of punch profile will modify the final shape of the part [88].

Based on the idea of controlling the strain distribution of the metal part, the Ohio State University developed a technology to directly apply the electromagnetic forces on the bottom region to increase the stretching of this region. The key idea is to embed the electromagnetic coil into the conventional stamping punch. These coils can be operated in a series of lower energy pulses occurring several times during the forming process. By proper integration of the EM coils with the forming press and tooling, this new method could dramatically improve the formability of sheet metal stamping.

\subsection{Experiment design and setup}

\subsubsection{Press and tooling}

A double-action hydraulic forming press was used to carry out all the experiments, as shown in Figure 6.1. In terms of mechanical configuration, the press features two actions: a punch and a blank holder. The punch has $978,560 \mathrm{~N}$ capacity and a 508mm stroke. The blank holder has a total force capacity of 1,334,400N [81].

The forming tooling was designed originally by Yuji Hishida and R. H. Wagoner for studying the effect of the BHF control forming method [81]. The set of tooling consists of a punch, a lower die, a blank holder and changeable draw beads to make scale-model fender panel. The dimensions of the fully formed panel are shown in Figure 6.2 [81]. The 
four corners of the panel have different combinations of punch and lower die radii and the four unsupported walls have different angles with respect to each other.

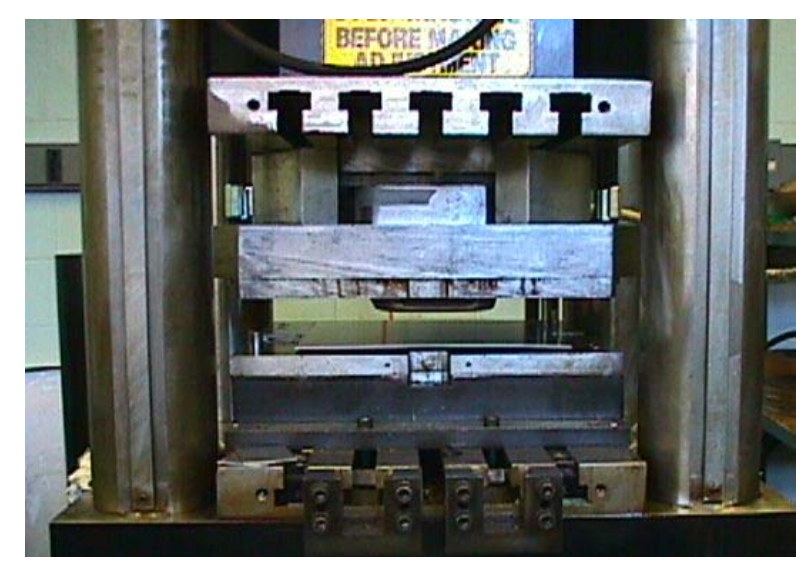

Figure 6.1 Photo of hydraulic press and tooling used in experiments

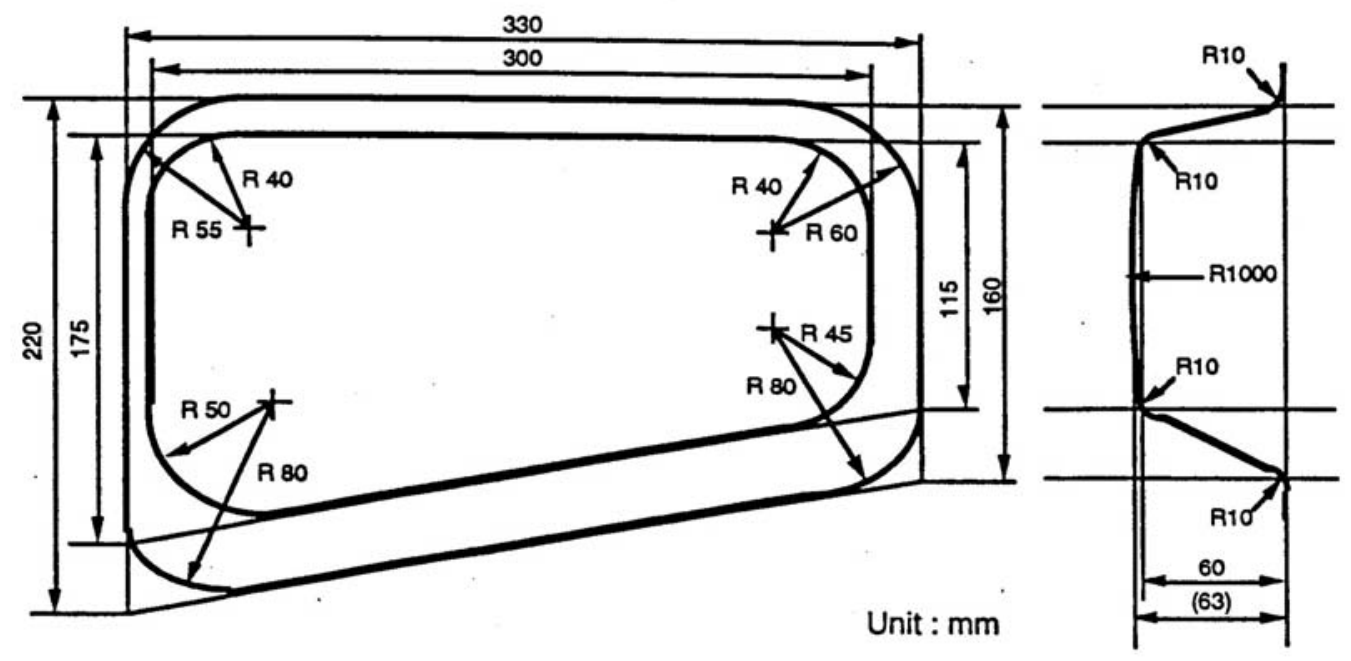

Figure 6.2 Dimensions of fully formed scale-model fender panel [81] 


\subsubsection{Electromagnetic coil and punch}

To increase the stretching of the bottom of metal part, electromagnetic coil should be embedded into the punch face close to the metal part. To embed the electromagnetic coil into the punch, a replicate punch was cast from a special iron filled epoxy. The punch then was grooved to accept the coil. The electromagnetic coil was fabricated from a brass bar to the appropriate shape and inserted into the punch. Kapton tape was used to electrically insulate the coil. Figure 6.3 is the photo of the punch with the electromagnetic coil embedded.

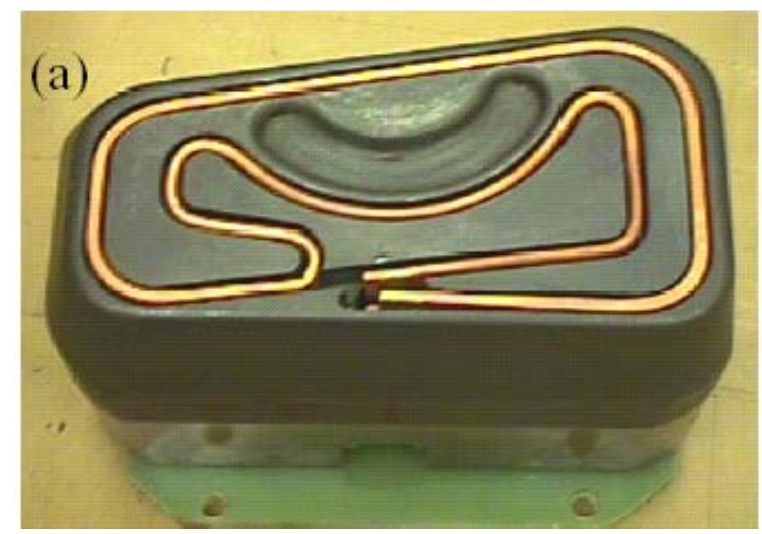

Figure 6.3 Photo of the punch and the embedded electromagnetic coil

Then the tooling was set up in the hydraulic double-action press. A flexible coaxial cable was used to connect the capacitor bank and the coil embedded in the punch, as shown in Figure 6.4. This cable was designed to carry current pulses from the capacitor bank to the coil. The current pulses flow through the coil and induce the eddy current in the nearby 
metal sheet. The primary current and the induced current produce the repulsive electromagnetic force, which will deform the metal sheet. A $48 \mathrm{~kJ}$ Maxwell Magneform capacitor bank was connected with the electromagnetic coil to supply the current pulses.

One important issue is to ground the press frame. Since the hydraulic press frame is made of steel, the press frame may have high potential and ground loop during the electromagnetic forming, which can damage the electronic control system. To avoid ground loop, single-point grounding was used by connecting the grounding wire from the capacitor back to the press frame. The whole experiment setup is shown in Figure 6.5.

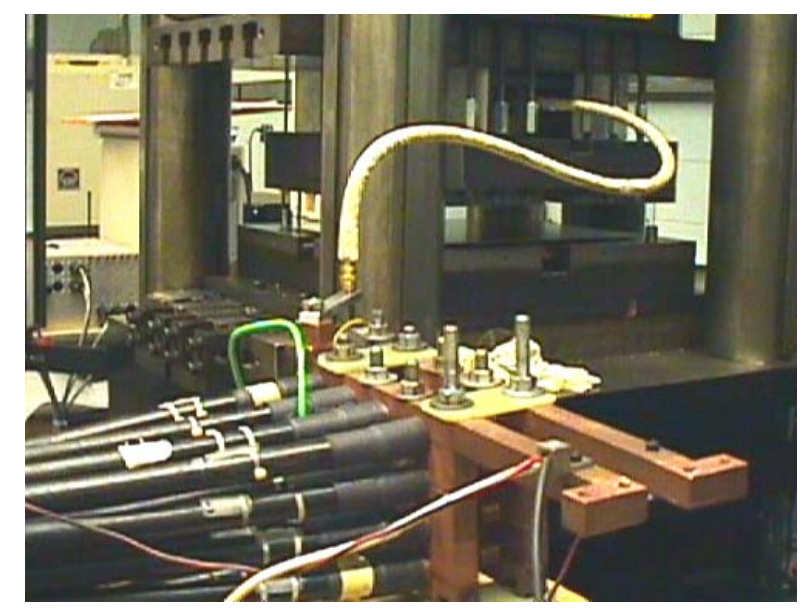

Figure 6.4 Connection between capacitor bank and electromagnetic coil inside of punch 


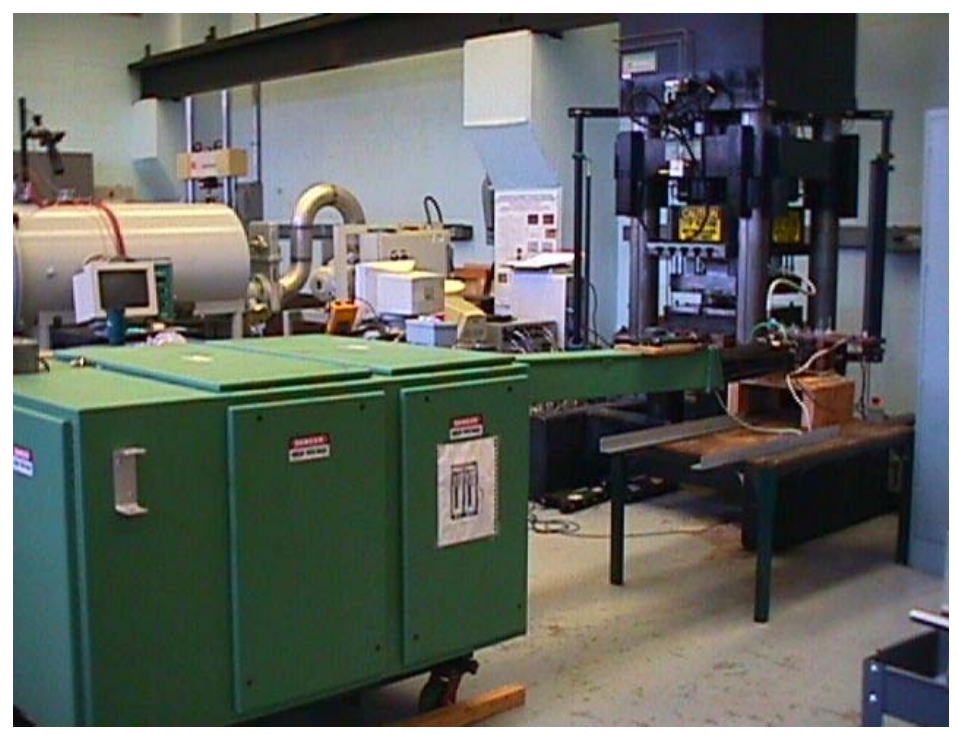

Figure 6.5 Experiment set-up (capacitor bank and press)

\subsubsection{Experiment procedure}

Several conventional stamping experiments were carried out to find the optimal forming conditions for the maximum draw depth. The results show that for 1mm-thick Al 6111T4 blank, the optimal forming conditions are constant 156KN clamp load with 4 draw beads at the four straight sides. The lubricant is 6Q oil which is only applied on the die regions. In above optimal conditions, the maximum draw depth without wrinkling was reached to 44mm for 1mm-thick Al 6111-T4 blank.

Electromagnetically assisted sheet metal stamping was carried out in the same forming conditions as above except the lubrication, to research the effect of the electromagnetic forces on the draw depth. During the forming process, several electromagnetic pulses 
with small energy level were applied to stretch the bottom region below the punch face. The schematic procedures for both conventional and electromagnetically assisted stamping are shown in Figure 6.6.

(a)
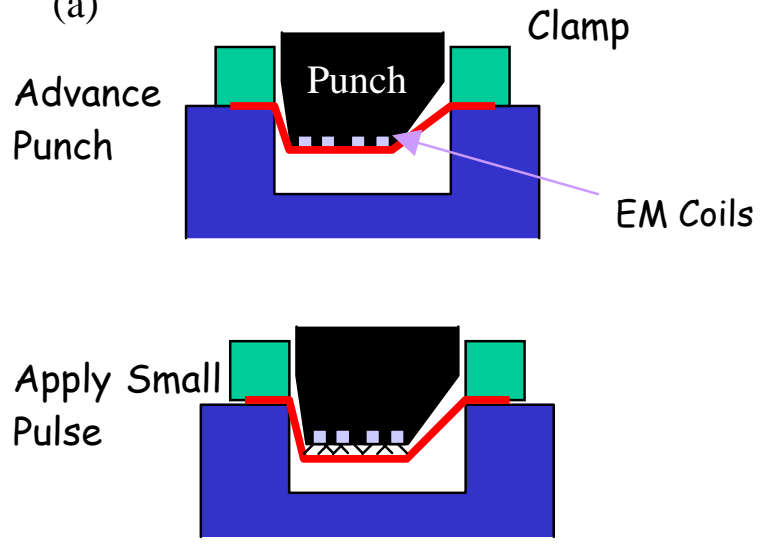

Advance

Punch

again (b)
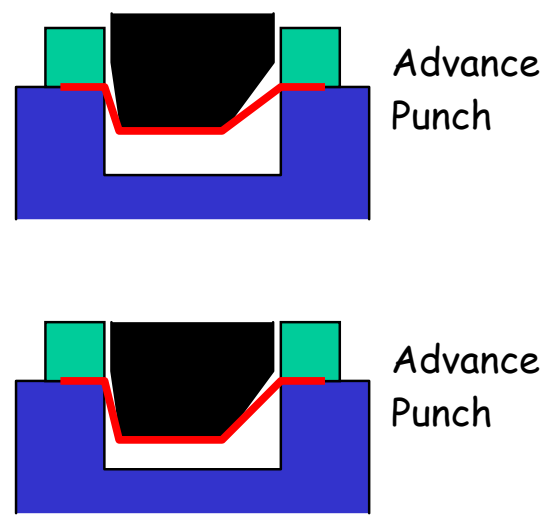

Figure 6.6 Schematic procedure of (a) electromagnetically assisted stamping; (b) conventional stamping

For conventional stamping experiments, the blank holder clamps the flange to prevent wrinkling and the punch is advanced to draw the metals into die cavity until the part cracks. While, for electromagnetically assisted stamping experiments, the blank holder 
also clamps the flange to prevent wrinkling. The punch is advanced a pre-decided amount between electromagnetic pulses to draw the metals into die cavity. The electromagnetic force stretches the bottom region with a small electromagnetic energy pulse. The punch is then advanced incrementally with each electromagnetic pulse until the part fails. No lubrication was used in these trials.

In this case, it is difficult to measure the stretching caused by EM forces, because the bottom region is big and not flat after EM pulses. So a different method was applied to determine the punch advance. The punch load should increase with the punch advance to stretch the bottom region of metal part. So the punch advance procedure was set to make the punch load increase after each EM pulse. The exact punch advance is relative to the EM energy of each pulse. The larger the EM energy is, the deeper the punch can advance after every EM pulse. While, larger EM energy requires robust tooling and may stretch the bottom region too much.

After many experiments, the optimal condition for this case was $5.4 \mathrm{~kJ}$ per EM pulse. And the punch advance procedure was to advance punch to $25.8 \mathrm{~mm}$ and then give the first EM pulse with $5.4 \mathrm{~kJ}$. From $25.8 \mathrm{~mm}$ to $46 \mathrm{~mm}$ of the draw depth, the punch was to advance a displacement of $2.53 \mathrm{~mm}$ between the EM pulses. After $46 \mathrm{~mm}$ of the draw depth, the punch was to advance a displacement of $1.76 \mathrm{~mm}$ between the EM pulses. In this procedure, the highest draw depth was achieved, comparing other experiment conditions. 


\subsubsection{Materials and lubrication}

The materials used in the experiments were Al 6111-T4 with 1mm thickness. The lubricant was 6Q lubricant.

\subsection{Experimental results}

\subsubsection{A typical result}

Below is a typical result produced by electromagnetically assisted stamping, comparing with the typical result of conventional stamping. Both of them were stamped with a constant $156 \mathrm{kN}$ clamp load. The right one was applied $22 \mathrm{EM}$ pulses with 5.4KJ each pulse. The results show that electromagnetic pulses did dramatically increase the draw depth without any reliance on lubrication. The draw depth increased by $44 \%$ (from $44 \mathrm{~mm}$ to $63.5 \mathrm{~mm})$.

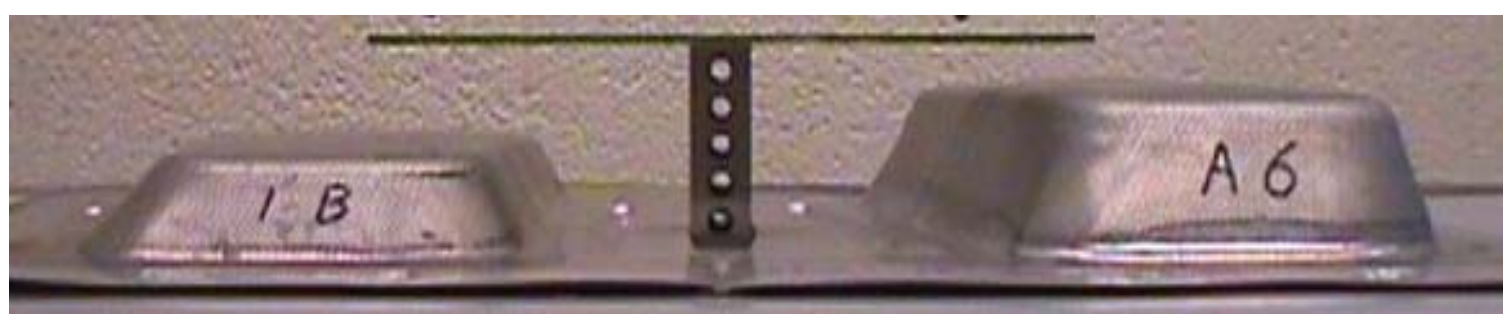

Figure 6.7 Photos of experiment results (Left: typical result made by conventional stamping; Right: typical result made by electromagnetically assisted stamping) 
The curves of punch load versus draw depth for above two samples are shown in Figure 6.8. The results of Figure 6.8 show that the punch load increased with the advance of the punch during the conventional stamping. For electromagnetically assisted stamping case, there was significant drop in punch load after each EM pulse. The reason of it is the stretching of the bottom region caused by EM forces. The repulsive EM forces between the coil and the metal sheet stretched the bottom region of the part and caused the force reduction.

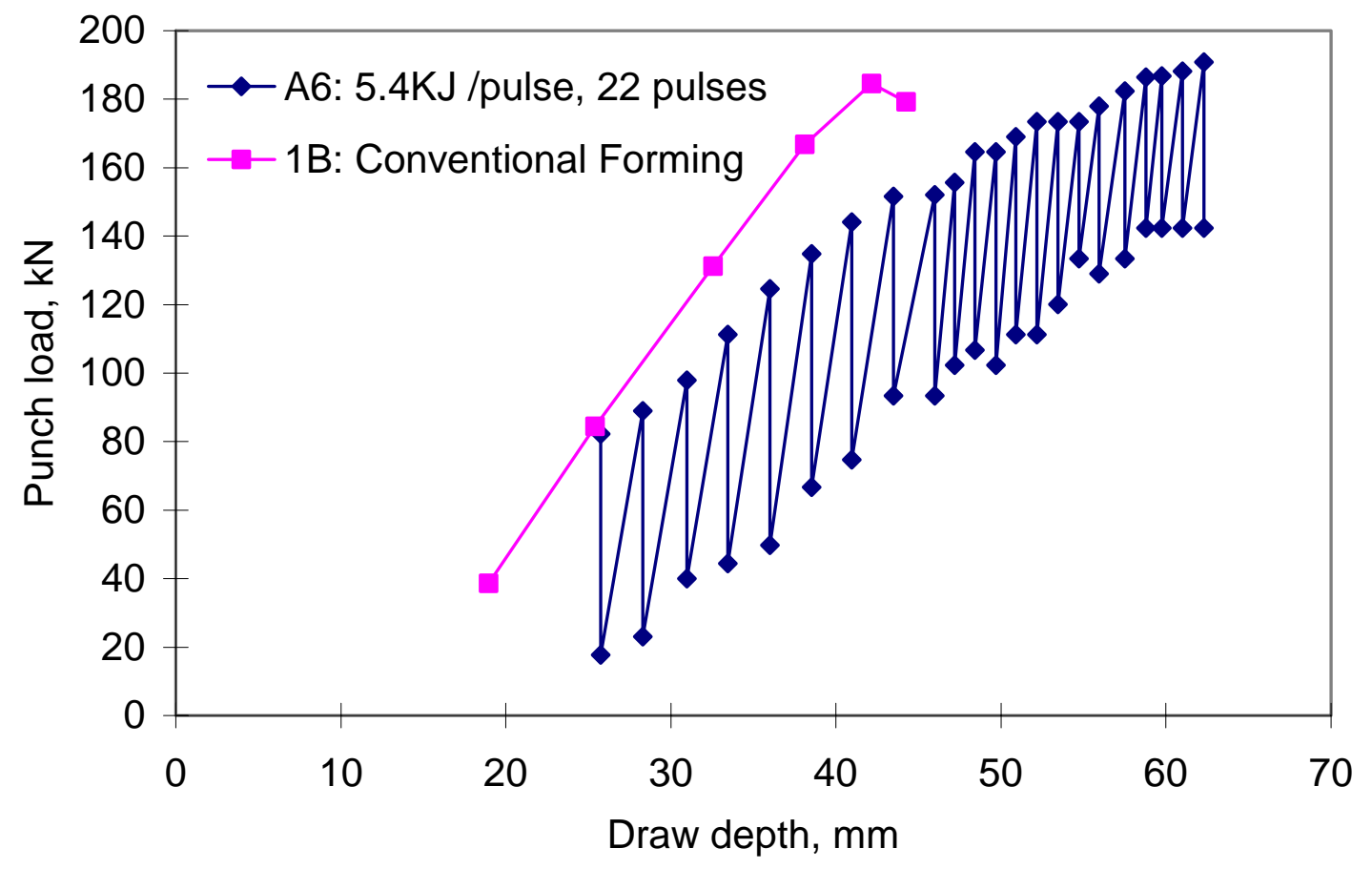

Figure 6.8 Punch load curves for the two samples shown in Figure 6.7 


\subsubsection{Strain distribution}

The EM pulses were designed to increase the stretching of the bottom region. To check if this goal was achieved, the strain distributions along $\mathrm{x}$ and $\mathrm{y}$ lines were measured. The locations of $\mathrm{x}$ and $\mathrm{y}$ lines are shown in Figure 6.9.

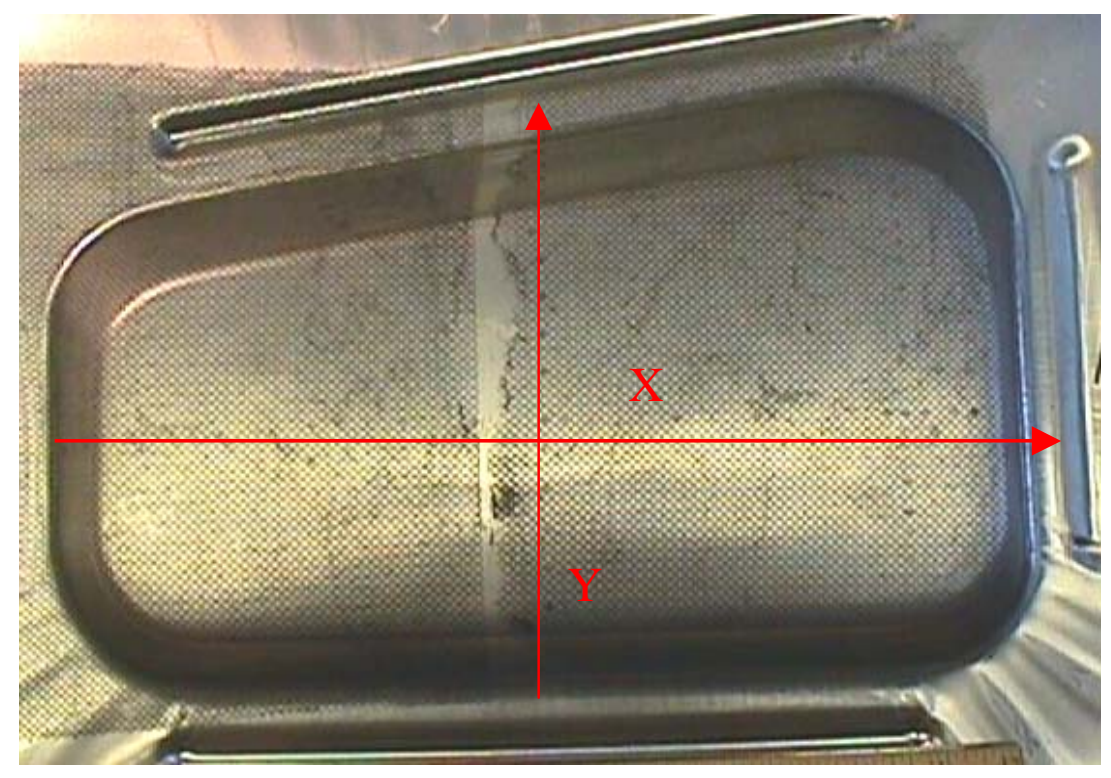

Figure 6.9 Locations of $\mathrm{x}$ and $\mathrm{y}$ lines on metal part

The comparison of the strains along y line is shown in Figure 6.10. And the comparison of the strains along $\mathrm{x}$ line is shown in Figure 6.11. Those results show that the bottom region did have relative large stretching. In conventional stamping, the bottom region has small deformation because of friction between the punch and the metal blank. While, for EM assisted stamping case, the bottom region has large deformation. The average major 
strain increased from 0.05 to 0.11 , about twice. And the draw depth increased by $44 \%$. But the strains at the side walls don't change much, because there is no EM force applied on those regions.

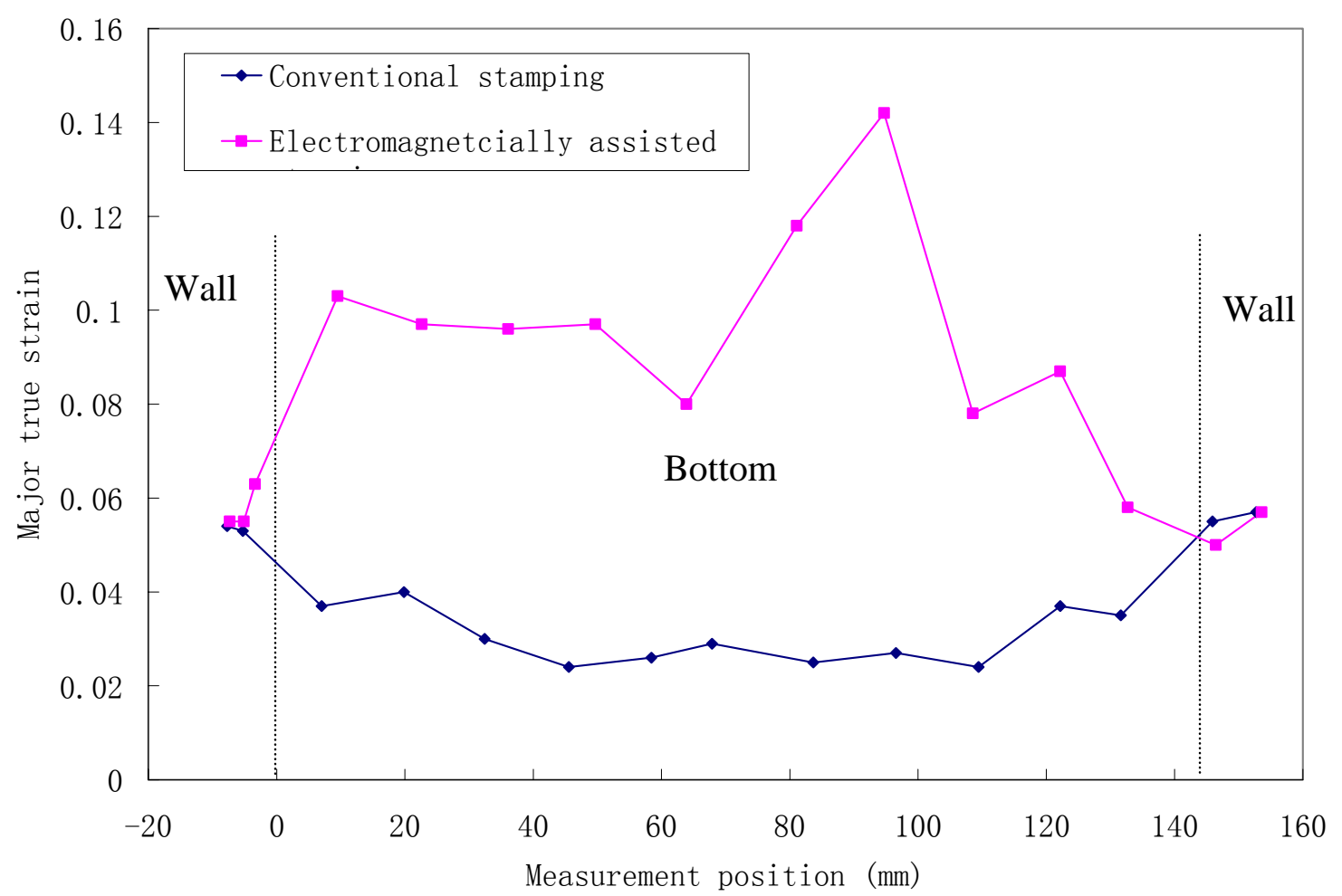

Figure 6.10 Strain distributions along the y line 


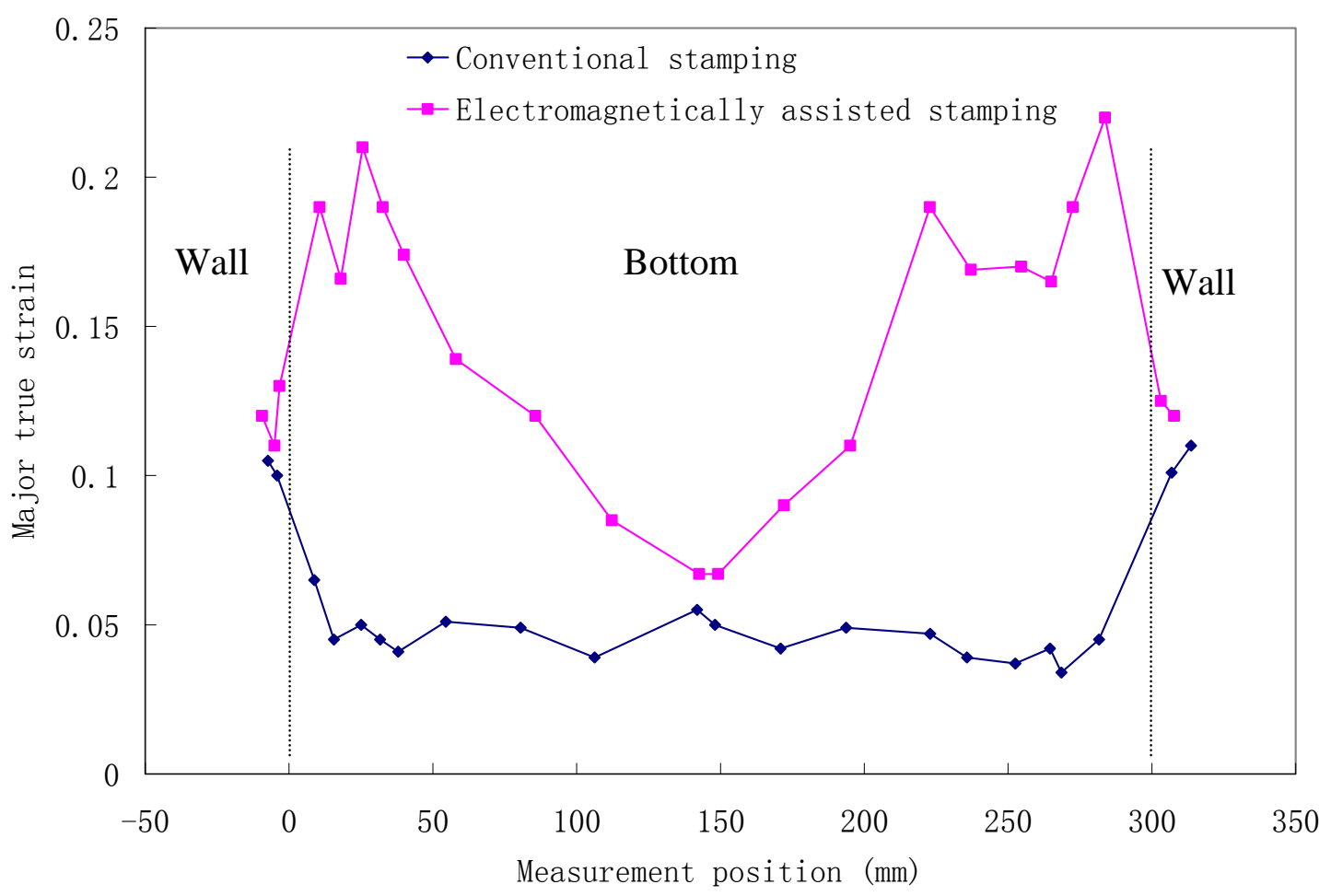

Figure 6.11 Strain distributions along the $\mathrm{x}$ line

Above strain distributions are at the bottom region and the straight side wall. The strain distributions were also measured at the corner region. The corner region has different strain states than the straight side wall. The straight side wall connects with the flange, which only involves bending and unbending when the flange is drawn into die cavity. While for corner region, the flange involves the flange shrink, which will provide more resistance for draw-in [88]. And tearing often happens at the corner near the punch nose. In this section, the strain distributions at the corner regions will be presented to investigate the effect of the EM forces. 
Figure 12 are the photos showing the measure lines at the corner regions. Figure 6.13 shows the strain distributions at the corner regions. It shows the strain states in EM assisted stamping are quite different from those in conventional stamping. At the bottom region, the strain states in former one are large bi-axial stretching. While, in later one, the strain states are small bi-axial stretching. At the area of the side wall near the punch nose, the strain states are close to plane strain state in conventional stamping and there is a tearing. For EM assisted stamping, the strain states at that region are bi-axial stretching and there is no tearing. At the lower half of the side wall, the strain states in both cases are tension-and-compression states. The hoop strain is compressive and the radial strain is tensile.

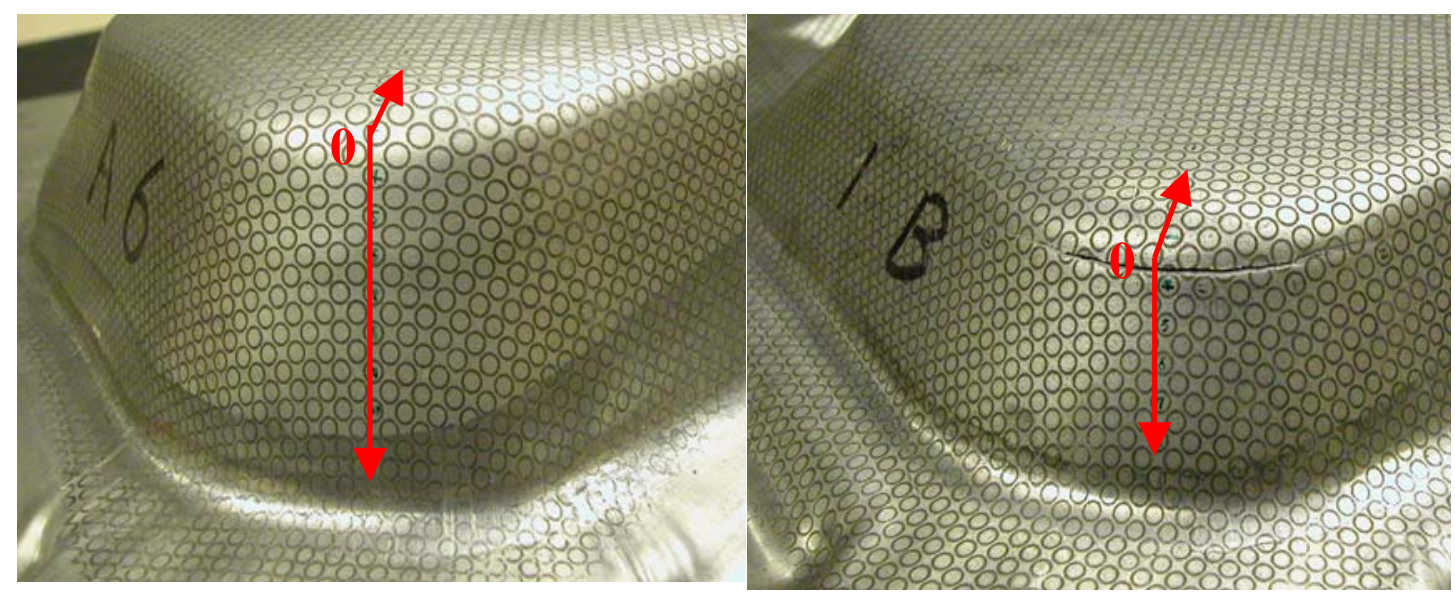

Figure 6.12 Photos of the corner regions and the locations of measure lines (Left: electromagnetic assisted stamping; Right: conventional stamping) 


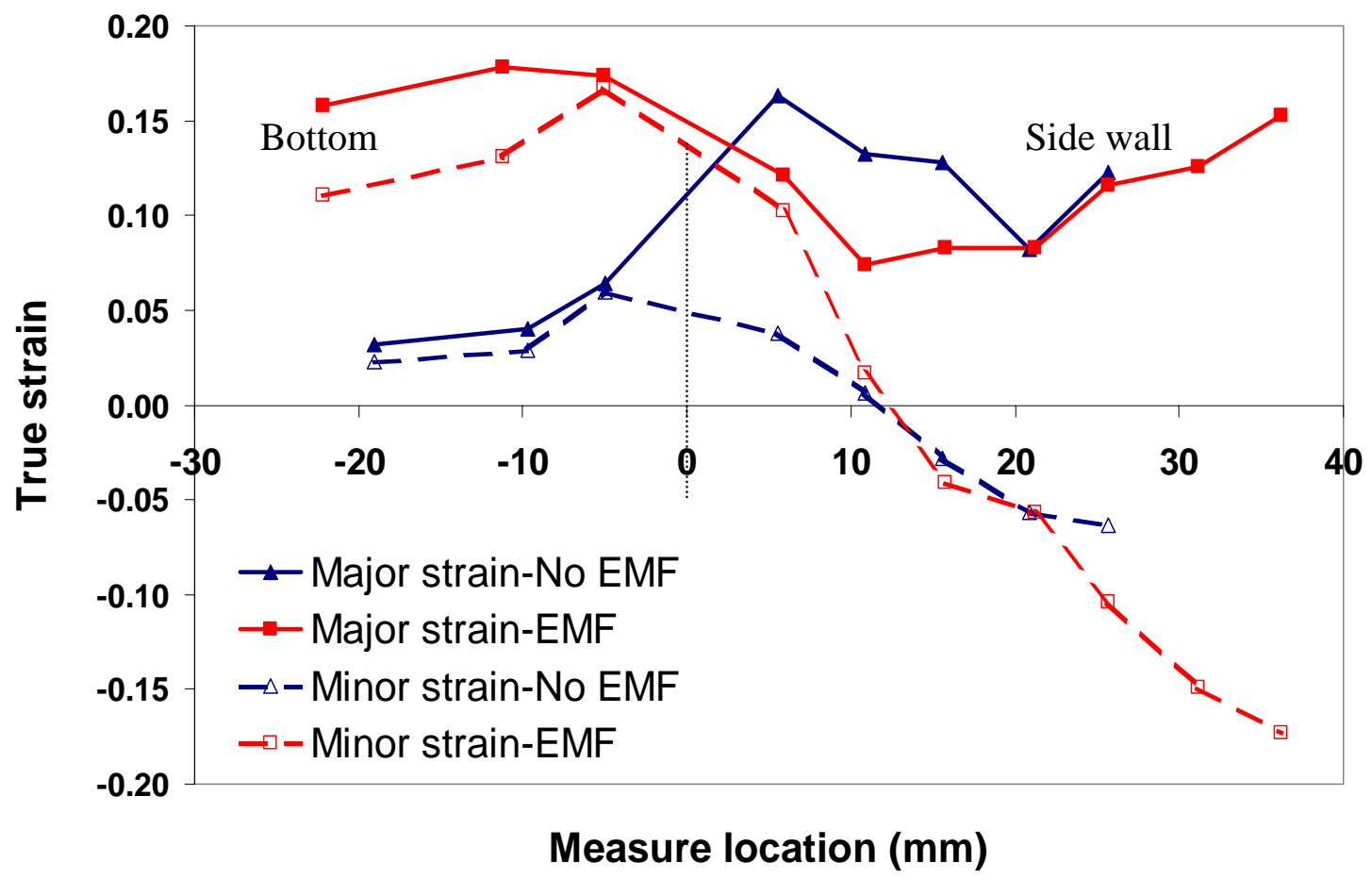

Figure 6.13 Comparison of strain distributions of corner region

The strain states of above two cases are also plotted in a Forming Limit Diagram for 6111-T4 aluminum [6]. The FLD data is from the ALCOA and reproduced in Figure 6.14. From the figure, it can be observed that there is a large strain state close to plane strain state in conventional stamping which is near the tearing position. While for the EM assisted stamping, the strain state are far into the two sides of the FLD. Thus, the blank can sustain higher magnitudes of major and minor strain in EM assisted stamping, compared to the one in conventional stamping. 


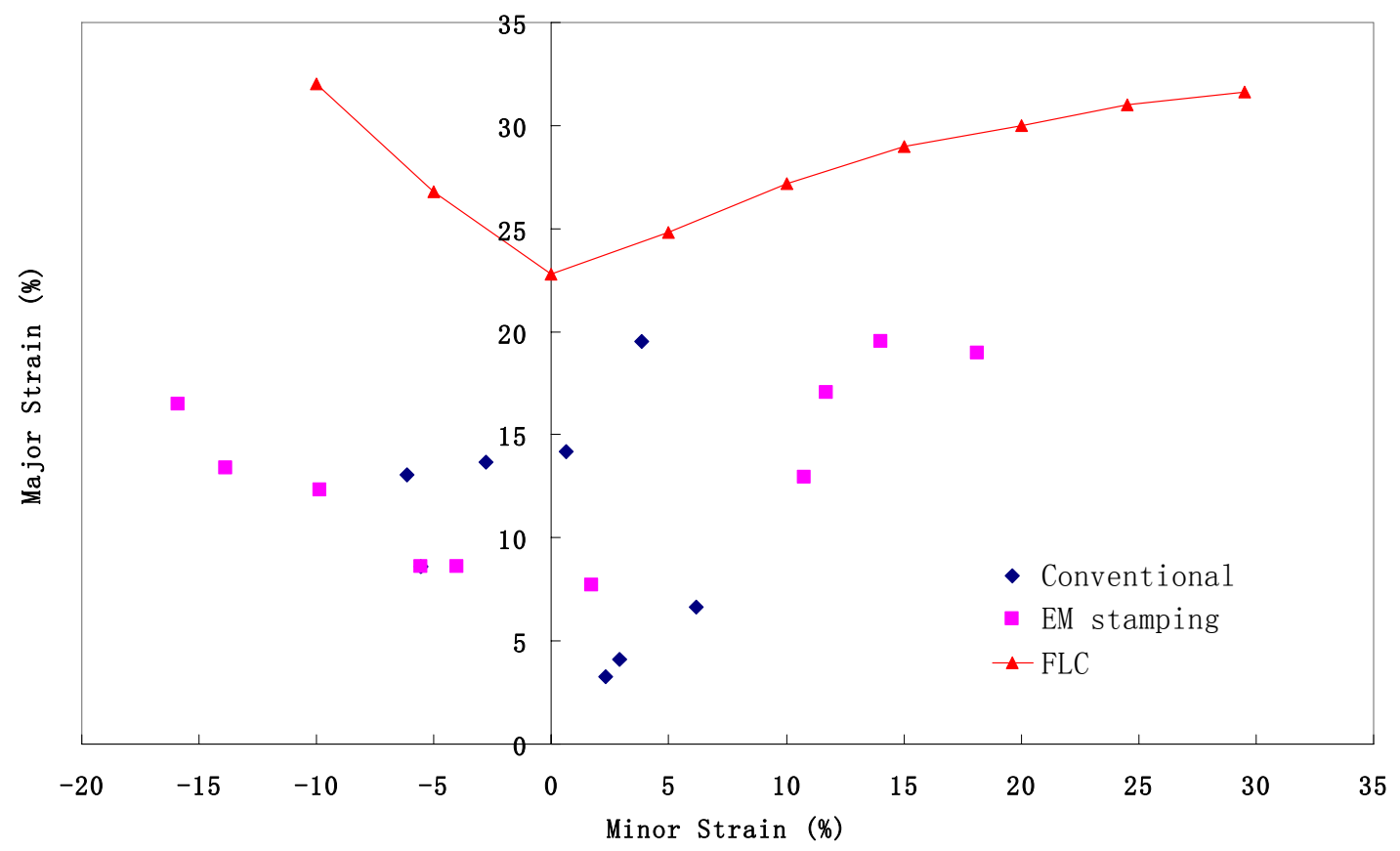

Figure 6.14 Measured strain states for both cases, compared to FLD for 6111-T4 [6]

\subsubsection{Stretching and draw-in}

Above section compared the strain distributions of the two cases. It clearly shows that EM forces do improve the stretching of the bottom region. Besides the stretching, the draw-in from the flange region is also improved dramatically.

Figure 6.15 and 6.16 are the photos of the straight and corner side walls for conventional and EM assisted stamping. Above figures clearly show that both the draw-in from the flange into the straight and corner side walls were dramatically improved in EM assisted stamping. 


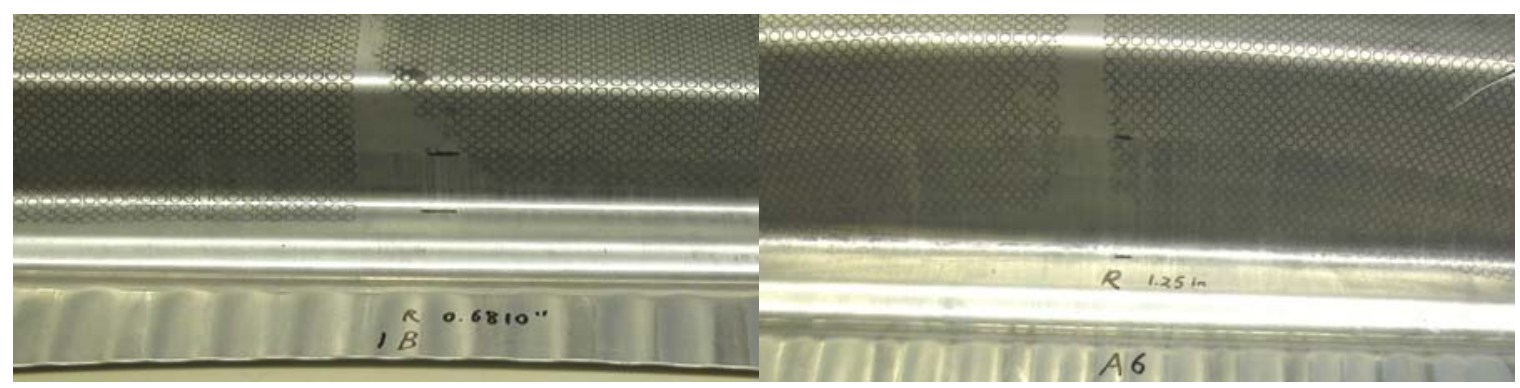

Figure 6.15 Photos of the straight side wall for conventional and EM assisted stamping

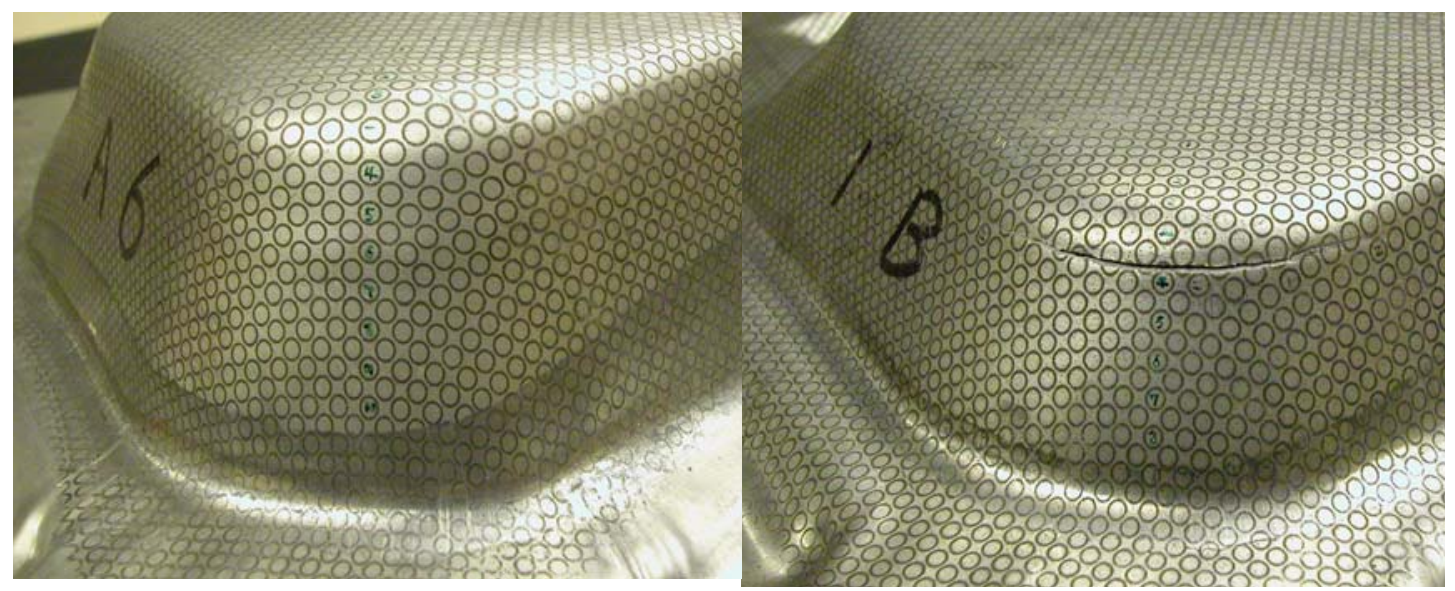

Figure 6.16 Photos of the corner side wall for conventional and EM assisted stamping

Figure 6.17 is about the comparison of the improvement of draw-in and the contribution of draw-in to side wall improvement at different locations. And the Table 6.1 is the summary of the improvement of draw-in and the improvement of side wall length for different sides. 


\begin{tabular}{|c|c|c|c|c|c|}
\hline & Corner 1 & Straight 1 & Straight 2 & Straight 3 & Straight 4 \\
\hline $\begin{array}{l}\text { Draw-in at conventional } \\
\text { forming, mm }\end{array}$ & 7.0 & 16.5 & 15.5 & 11.5 & 7.9 \\
\hline $\begin{array}{l}\text { Draw-in improvement at } \\
\text { EM assisted stamping, mm }\end{array}$ & 6.5 & 15.4 & 7.0 & 9.7 & 7.3 \\
\hline Improvement of draw-in & $92.8 \%$ & $93.3 \%$ & $45.2 \%$ & $84.3 \%$ & $92.4 \%$ \\
\hline $\begin{array}{l}\text { Side wall length } \\
\text { improvement, mm }\end{array}$ & 17.5 & 18.5 & 18.0 & 11.3 & 15 \\
\hline $\begin{array}{l}\text { Contribution of draw-in to } \\
\text { side wall improvement }\end{array}$ & $37.1 \%$ & $83.2 \%$ & $38.9 \%$ & $85.8 \%$ & $48.7 \%$ \\
\hline
\end{tabular}

Table 6.1 Summary of improvement of draw in and side wall length at different sides

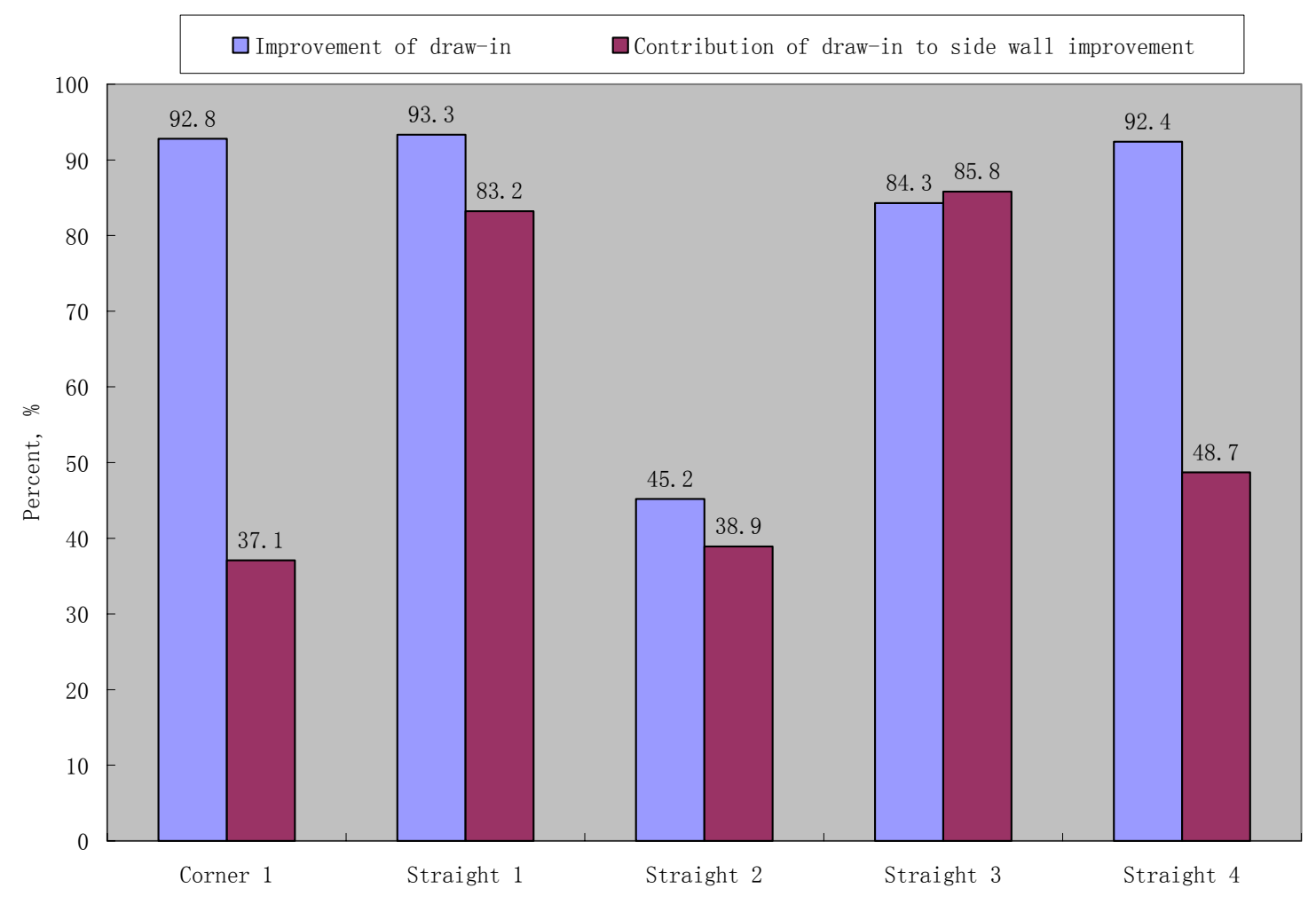

Figure 6.17 Comparison of improvement of draw-in and contribution of draw-in 


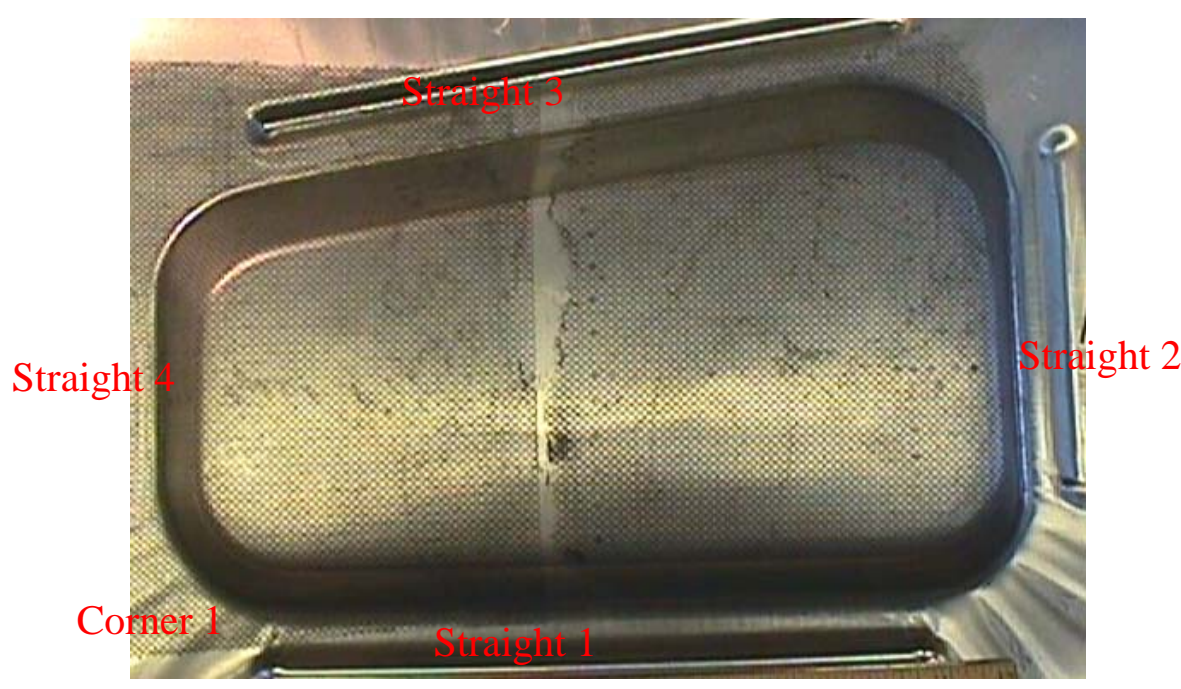

Figure 6.18 Different locations shown in Figure 6.17

It should be noticed that the amounts of improvement of draw-in are different for different locations. And the contributions of draw-in for the improvement of length of side walls are also different. It is more difficult to draw metals from the flange at the corner than the straight sides, because the corner flange involves the shrink deformation and needs extra forces to draw in. From the Figure 6.17, it can be seen that the draw in at the corner provides the least contribution to the improvement of side wall length, which means more metal at the corner was converted from the bottom region into the side wall.

\subsubsection{Stretching only}

In this section, the effect of stretching only will be discussed. To prevent the draw in from the flange, a very high clamp load (1245 kN) was applied during both the conventional and EM assisted stamping. And no lubricant was used. For EM assisted 168 
stamping, the energy level was $5.4 \mathrm{~kJ}$ per pulses and $21 \mathrm{EM}$ pulses were applied. The results are shown in Figure 6.19. There is different failure mode from the one with lower clamp load $(156 \mathrm{kN})$. With this high clamp load, the tearing happened at one of the straight side wall, instead of the corner side wall near punch nose. And for EM assisted case with high clamp load, there was a tearing at the bottom region.

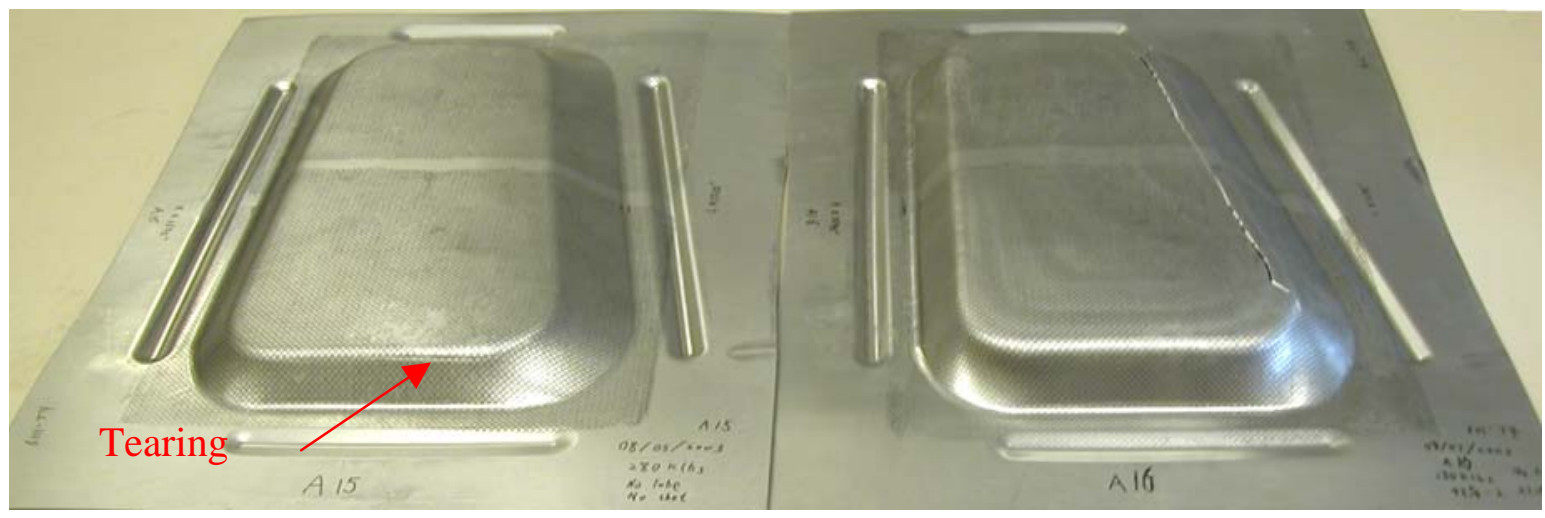

Figure 6.19 Photos of the samples with $1245 \mathrm{kN}$ clamp load (Left: conventional stamping, draw depth is 25.3mm; Right: EM assisted stamping, draw depth is $31.0 \mathrm{~mm}$ )

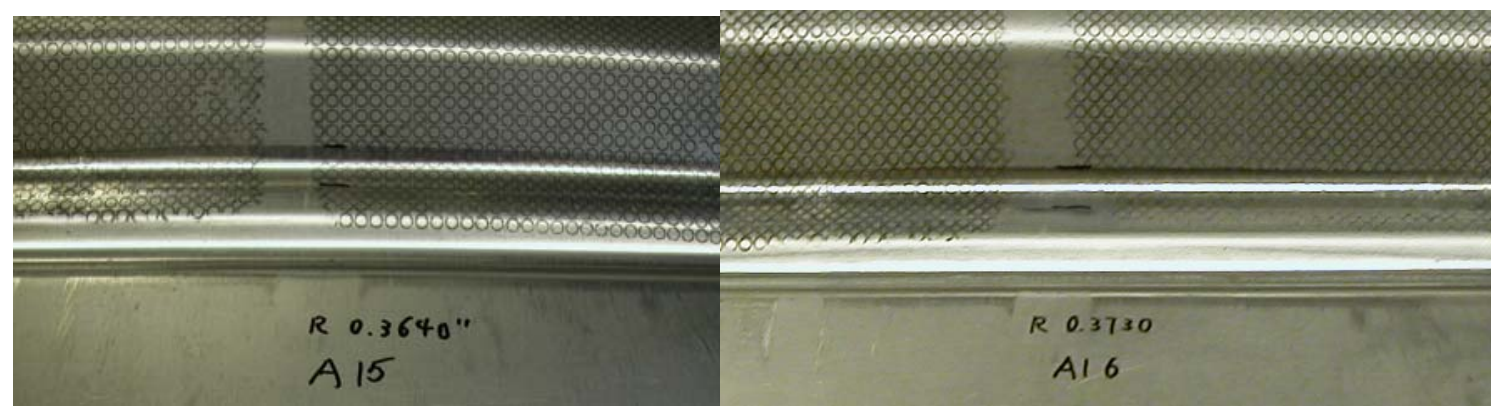

Figure 6.20 Straight side walls for conventional (left) and EM assisted stamping (right) 
The results clearly show that there was no improvement of draw in from the flange in EM assisted stamping, shown in Figure 6.20. The improvement of draw depth only came from the stretching of the bottom region. Therefore, with high clamp load, there was no improvement of draw in and the draw depth increased by 22\%. The stretching of the bottom region has the limits. It can not stretching too much. Otherwise, it will exceed the forming limits and cause failures. Figure 6.21 shows the punch load versus draw depth curves for above two samples. It can be seen that the peak punch load in EM assisted stamping was almost same as the one in conventional stamping, which means the EM pulses did not increase the flow stress of the sheet metal.

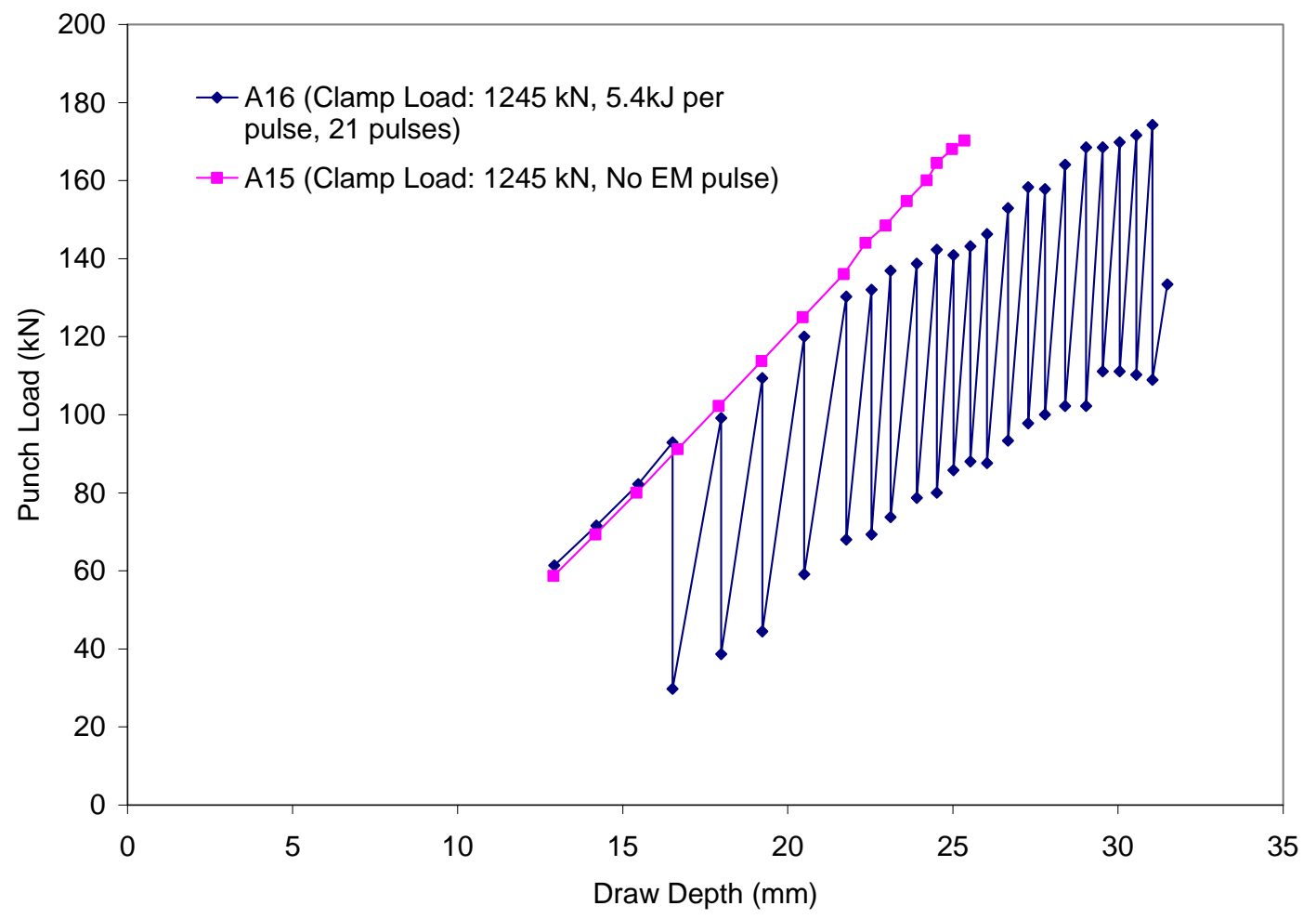

Figure 6.21 Curves of punch load versus draw depth 
The experiments with relatively high clamp load $(623 \mathrm{kN})$ were also carried out. The results show that there was also no improvement of draw in and the draw depth increased only by $21 \%$.

Figure 6.22 shows that to reach the maximum improvement of draw depth, a suitable clamp load is needed to get both the stretching of the bottom region and the draw in of the flange. Only stretching the bottom region has limited effective on the draw depth improvement.

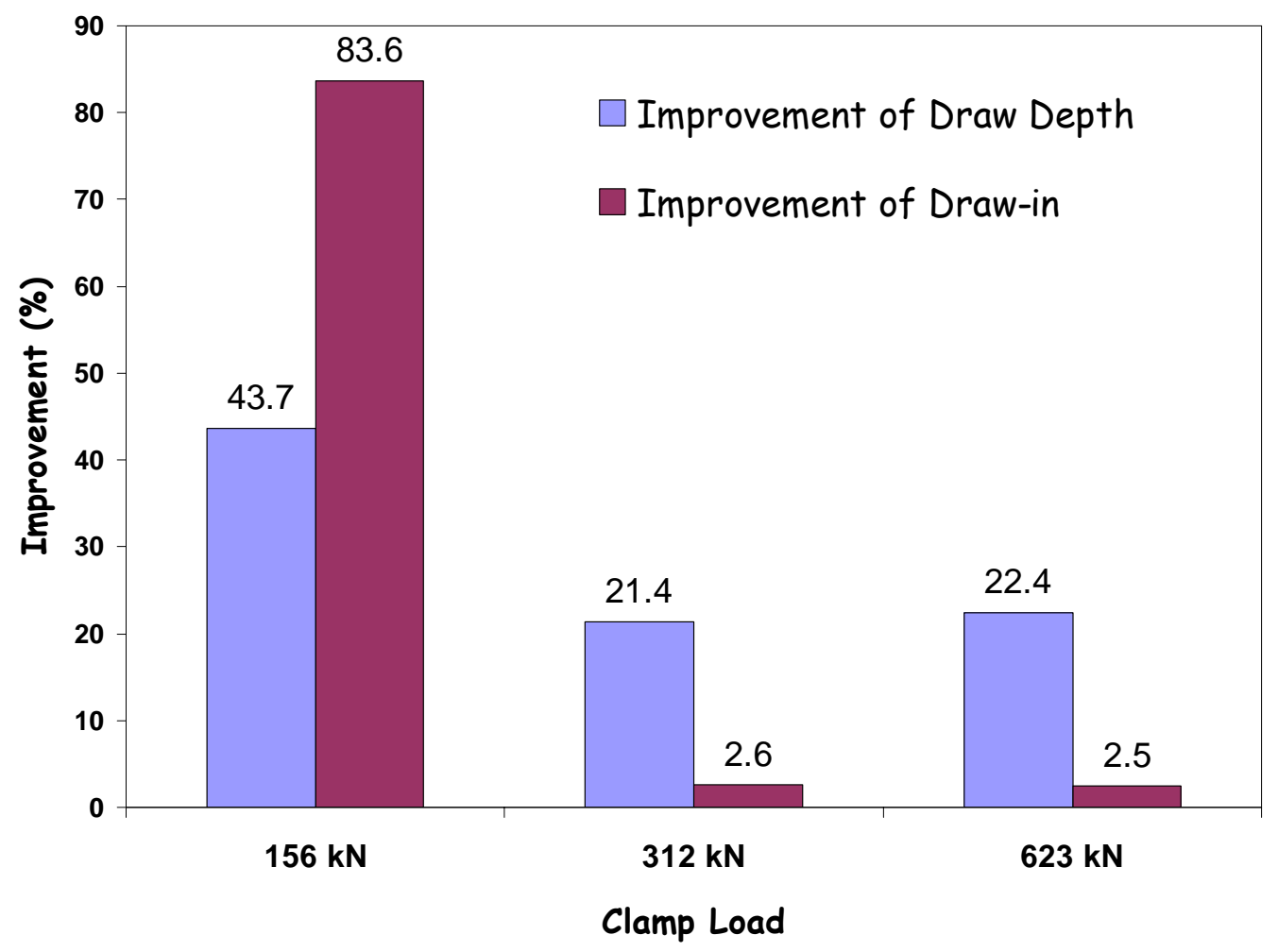

Figure 6.22 Comparison of effect of clamp load on draw in and draw depth 


\subsubsection{Effect of coil configuration}

EM coil is used to deliver repulsive EM forces to the metal sheet. Its configuration defines the forces distribution, which in turn directly effects the strain distribution of the bottom region. Two different coil configurations were used, as shown in Figure 6.23.

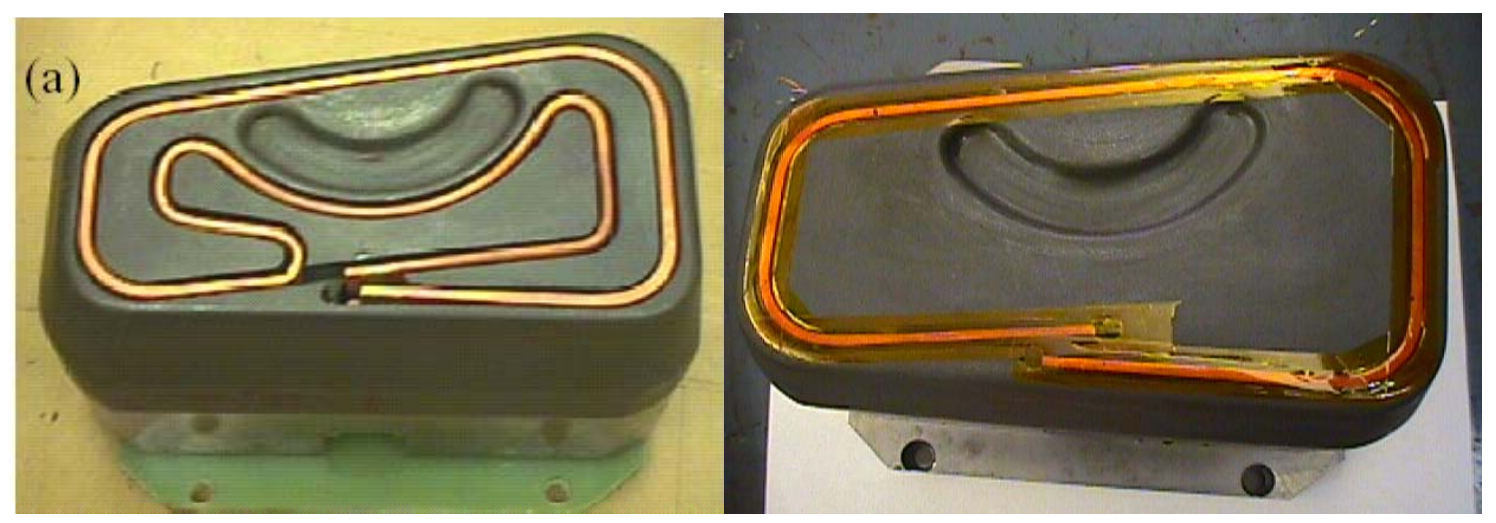

Figure 6.23 Photos of coil configurations (Left: configuration 1; Right: configuration 2)

The coil with configuration 2 was used with $0.8 \mathrm{~mm}$ thick Al 6111-T4 blank and $133 \mathrm{kN}$ clamp load. The EM energy level was 3.6kJ per pulse and 55 pulses were applied. To compare with this sample, another sample of $0.8 \mathrm{~mm}$ thick Al 6111-TA blank was formed by conventional stamping with $133 \mathrm{kN}$ clamp load.

The strain distributions along $x$ line are measured and shown in Figure 6.24. Comparing to those with coil configuration 1 (Figure 6.11), the part has larger strains at the perimeter. And over much area of the bottom region of the metal part, there is very little 
increase in the strain level. But by the Configuration 1, there is significant increase in strains at much area besides area near perimeter. Because of the larger length of the coil beneath the top surface, electromagnetic forces can be applied to stretch more areas.

From the above strain distributions, it can be concluded that the draw depth with configuration 2 could not increase as much as the one with coil configuration 1, since the EM stretching of the bottom region happened at smaller areas. With coil configuration 2, the draw depth only increased by $15 \%$.

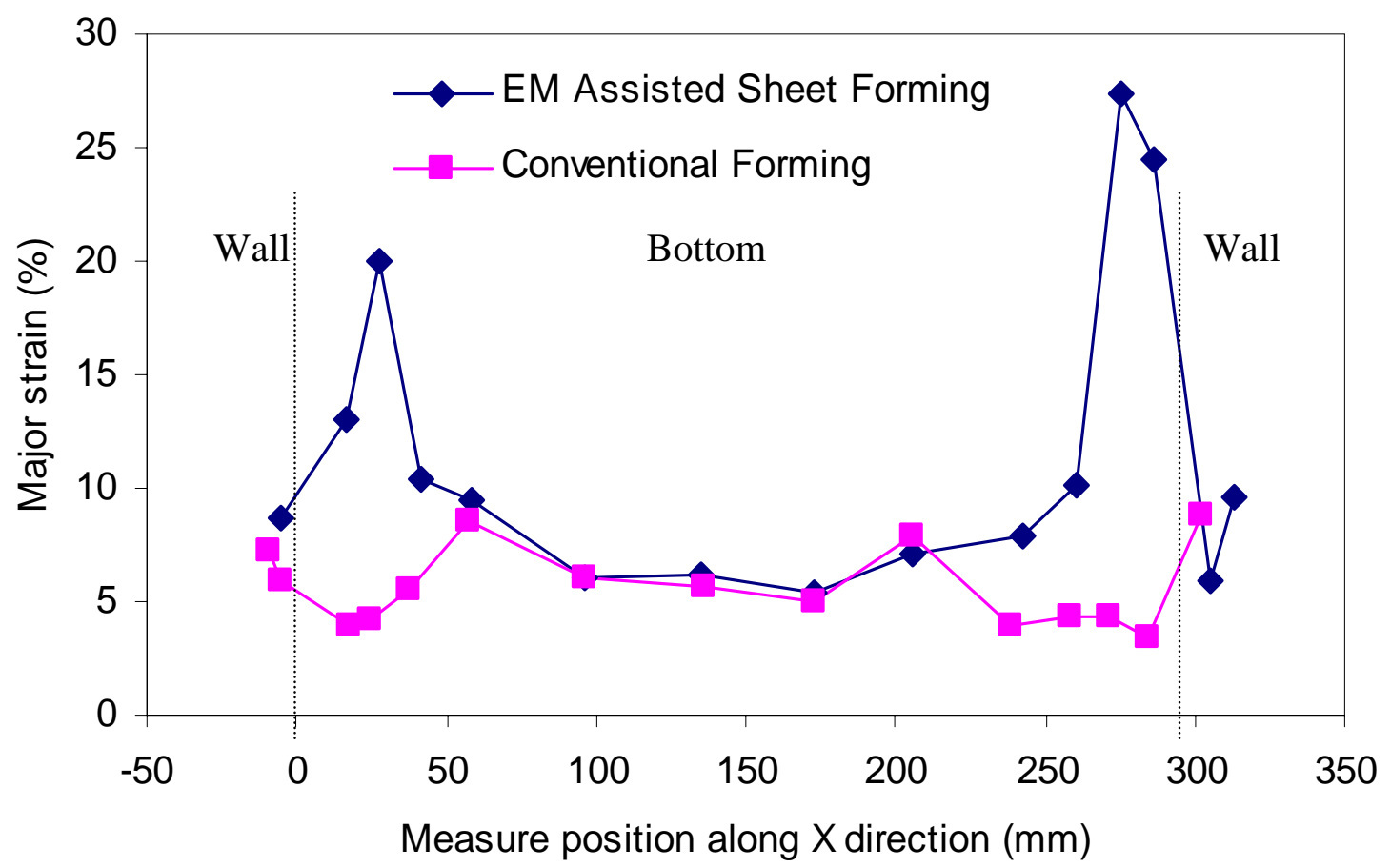

Figure 6.24 Strain distributions along $\mathrm{x}$ line with coil configuration 2 


\subsubsection{Effect of process parameters}

The process parameters, such as punch advance, electromagnetic energy, have great effect on the draw depth and the strain distribution as well as the propensity of the sample to wrinkle. Different combinations of punch advance, EM energy and clamp load were tried to reach the maximum draw depth.

For the deepest draw depth, the punch was advanced incrementally with each discharge, a displacement of $2.53 \mathrm{~mm}$ after each pulse until the punch load reached about $156 \mathrm{kN}$. Then punch advanced a displacement of $1.76 \mathrm{~mm}$ after each pulse until failure. And the EM energy was $5.4 \mathrm{~kJ}$ per pulse. By such approach, the deepest draw depth in this experiment is achieved.

During the experiments, it was found that the process parameters affect each other. For different punch advances, different EM energy levels should be applied. And for different

clamp load, the different punch advance procedures should be used. The EM assisted stamping is sensitive to the process parameters. For different applications, detail process parameters should be determined according to the actual situations.

\subsubsection{Failure modes}

In conventional stamping, there are three kinds of failures: the wrinkling at the flange, the buckling at the side wall, and the tearing at the corner side wall near punch nose. While for EM assisted stamping case, besides above three kinds of failures, there is one new 
failure mode: the tearing at the bottom region. The tearing is just above the EM coil, and caused by the excessive stretching of the bottom region with EM pulses.

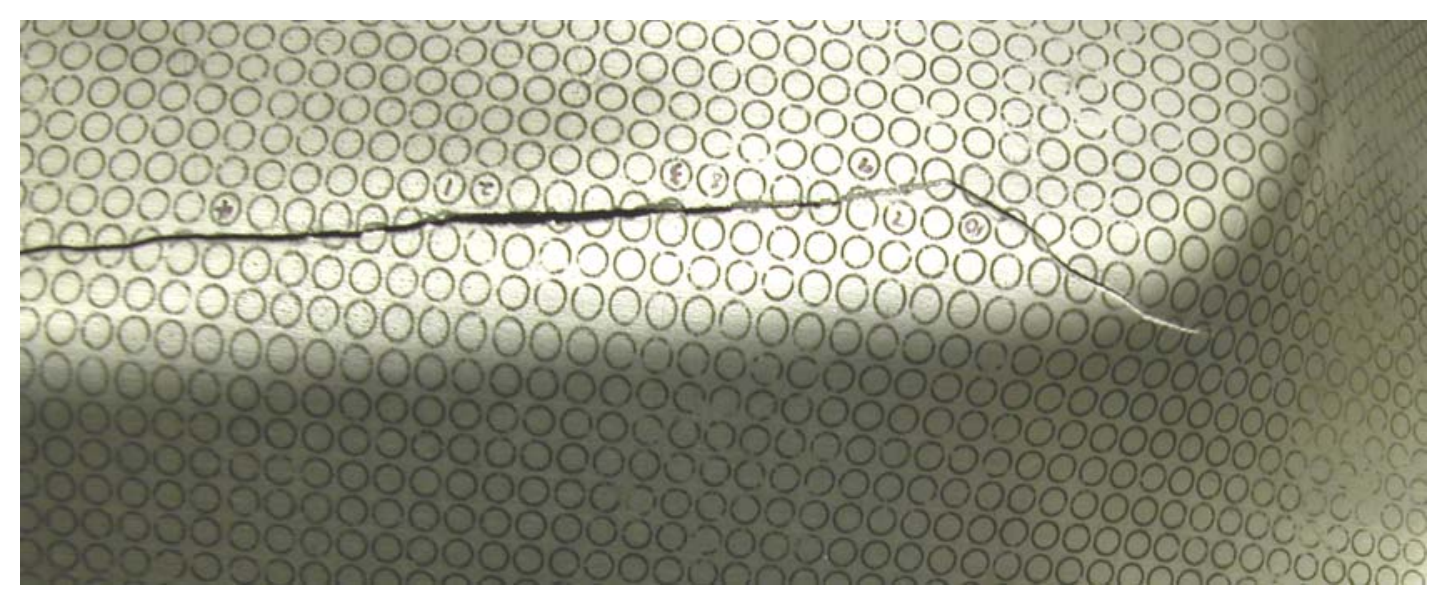

Figure 6.25 Tearing at the bottom region

It's said that high velocity forming can increase the forming limits of metals [53-56]. Although in this experiment the small EM energy level was used, the forming velocity may still be high. To check if the forming limits are increased, the strain states were measured near the tearing at the bottom region caused by EM pulses. The conventional forming limits data of AA 6111-T4 are from the Dr. Vohnout's dissertation [6]. The results shown in Figure 6.26 clearly indicate that the strain states are still very close to the conventional forming limits. It can not be seen that the strain limits in EM assisted stamping are increased. 
In EM assisted stamping, we did not expect to improve the strain limits. The goal of EM forces is to control the strain distribution to improve the formability of stamping. The experiment results show that the goal was achieved.

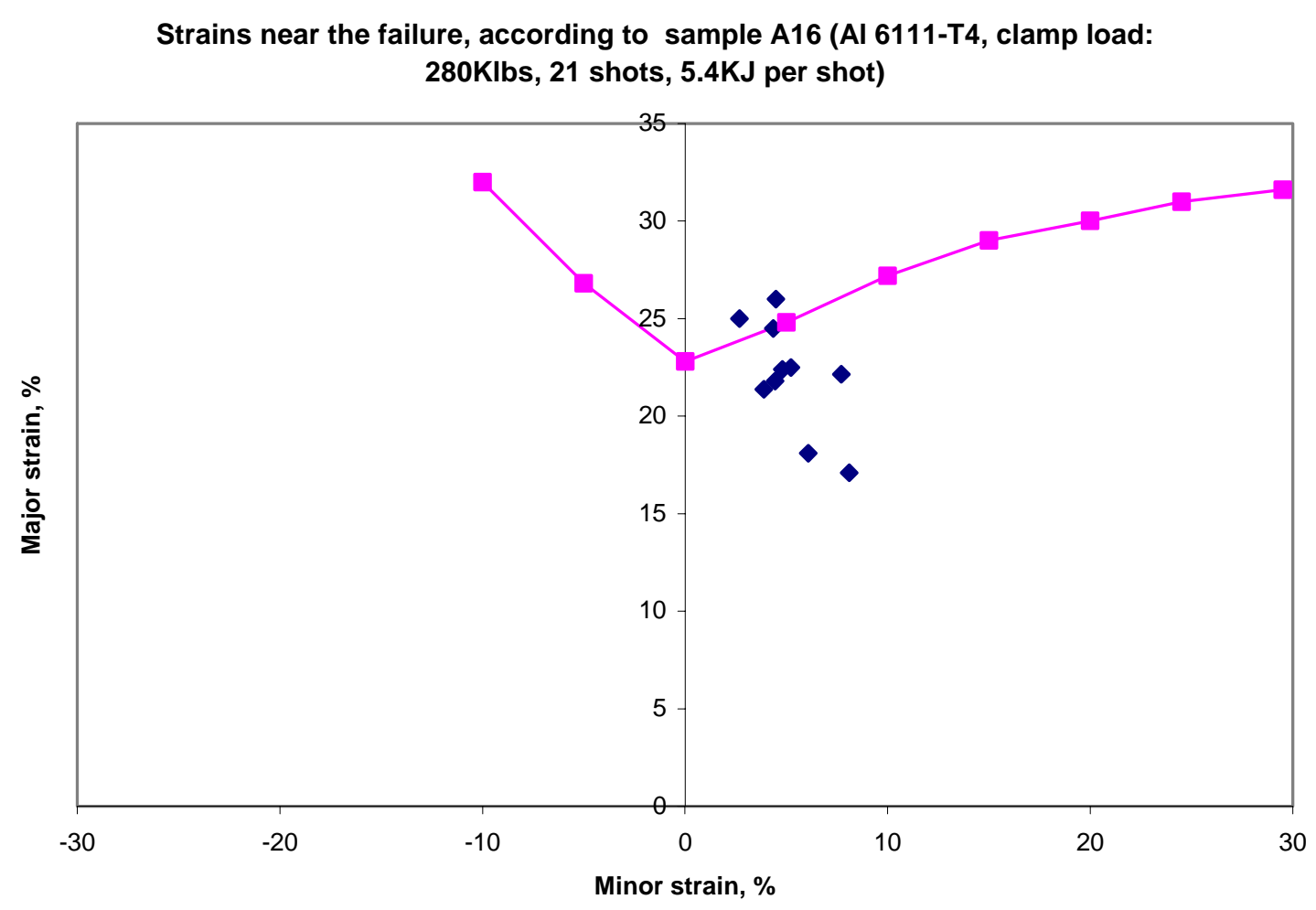

Figure 6.26 Measured strains near the crack, compared to FLD for Al 6111-T4 [6]

\subsubsection{Bottom region waviness}

The shortcoming of the EM assisted stamping is the waviness at the bottom region. Because of the EM pulses, the metals at the bottom region are pushed up to stretch. Since the EM forces are not uniformly distributed on the bottom region, some areas have large 
EM forces and some areas have little EM forces. Large EM forces push the metals near the coil far away from the punch surface. Therefore, the waviness of the bottom region occurs. Figure 6.27 are the scanned surface shape of the bottom region along $x$ line for the conventional and EM assisted stamping. For conventional stamping, the bottom region is smooth. While, for EM assisted stamping, there are two areas much higher than the rest of areas.

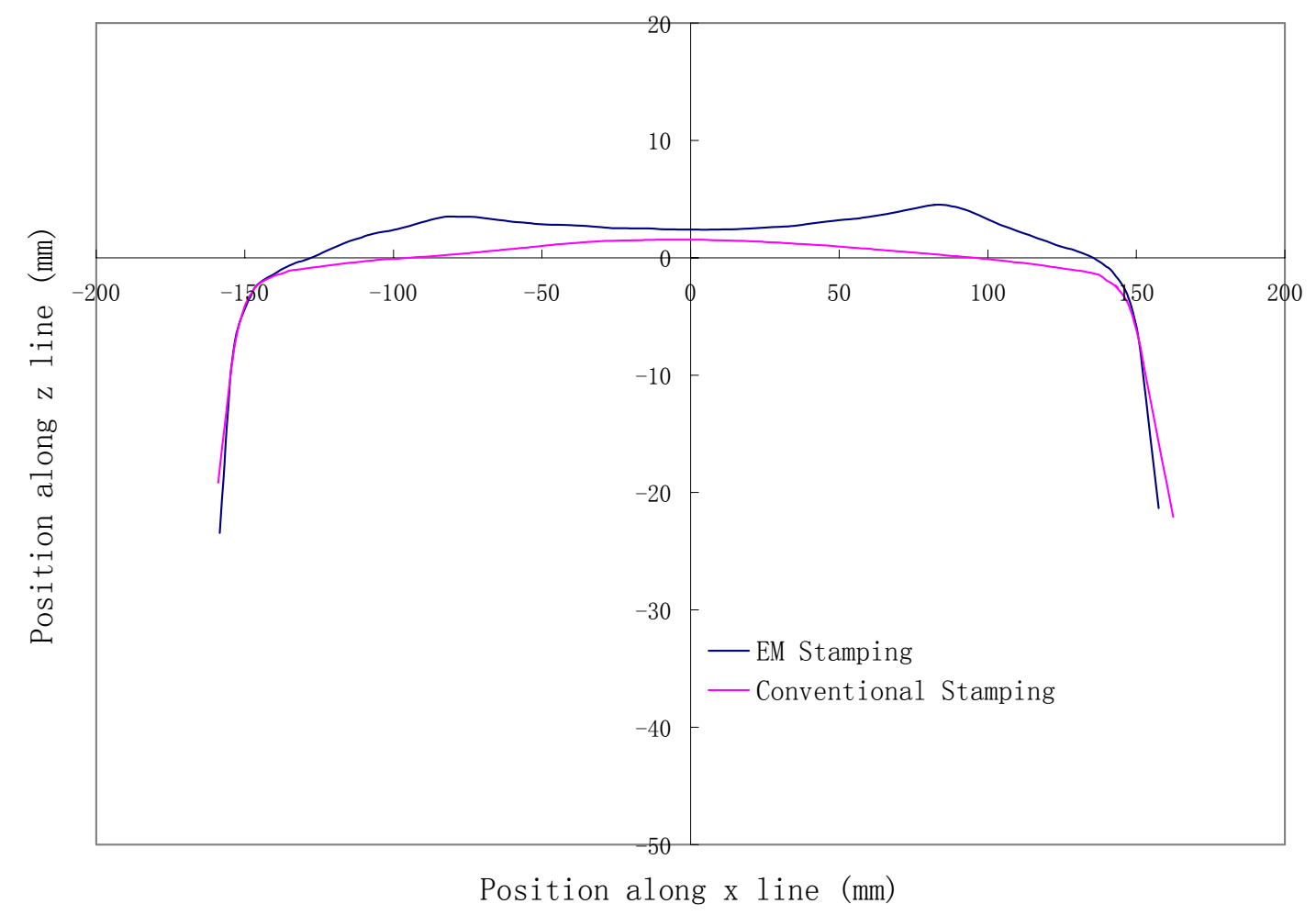

Figure 6.27 Scanned surface shape of bottom region along $x$ line 
To eliminate the waviness, a coil to provide uniformly distributed EM forces is needed. Until now, such kind of coil is still in research. To reduce the waviness, multi-turn coil can be considered. The basic rule is to increase the coil length and make the coil gap smaller. Hence more areas of bottom region can be affected by EM forces. In this way, it could make the stretching of the bottom region more uniform and reduce the waviness.

\subsection{Theoretical analysis}

In the theoretic analysis, materials is assumed to be rigid-plastic and the strain hardening characteristics is of the form [88],

$$
\bar{\sigma}=K(\bar{\varepsilon})^{n}
$$

where $\mathrm{K}$ is the material constant, $\bar{\sigma}$ is the flow stress, $\bar{\varepsilon}$ is the flow strain and $n$ is the strain hardening exponent. The material properties are assumed to be isotropic in the sheet plane so that there is only a normal anisotropy $\bar{R}$.

Material is assumed to be yielding according to Hill's anisotropic criterion and expressions for flow stress $\bar{\sigma}$ and increments strain $d \bar{\varepsilon}$ [88] are given (neglecting shear components) by

$$
\begin{aligned}
& \bar{\sigma}=\sqrt{\frac{1}{1+\bar{R}}}\left[\left(\sigma_{1}-\sigma_{3}\right)^{2}+\left(\sigma_{2}-\sigma_{3}\right)^{2}+\bar{R}\left(\sigma_{1}-\sigma_{2}\right)^{2}\right]^{1 / 2} \\
& d \bar{\varepsilon}=\frac{\sqrt{1+\bar{R}}}{1+2 \bar{R}}\left[\left(d \varepsilon_{2}-\bar{R} d \varepsilon_{3}\right)^{2}+\left(d \varepsilon_{1}-\bar{R} d \varepsilon_{3}\right)^{2}+\bar{R}\left(d \varepsilon_{1}-d \varepsilon_{2}\right)^{2}\right]^{1 / 2}
\end{aligned}
$$

And then the flow rule [88] is given by 
$\frac{d \varepsilon_{1}}{\bar{R}\left(\sigma_{1}-\sigma_{2}\right)+\left(\sigma_{1}-\sigma_{3}\right)}=\frac{d \varepsilon_{2}}{\bar{R}\left(\sigma_{2}-\sigma_{1}\right)+\left(\sigma_{2}-\sigma_{3}\right)}=\frac{d \varepsilon_{3}}{\left(\sigma_{3}-\sigma_{2}\right)+\left(\sigma_{3}-\sigma_{1}\right)}=\frac{d \bar{\varepsilon}}{(1+\bar{R}) \bar{\sigma}}$

Here $\sigma_{1}, \sigma_{2}$ and $\sigma_{3}$ are stresses and $d \varepsilon_{1}, d \varepsilon_{2}$ and $d \varepsilon_{3}$ are strain increments in $x, y$ and $z$ directions respectively.

Generally the electromagnetic pulses finish the deformation in less than $100 \mu$, which leads to large strain rate. The constitutive equation below [88] should be applied

$$
\bar{\sigma}=K \bar{\varepsilon}^{n}(\dot{\varepsilon})^{m}
$$

While, for aluminum alloys in room temperature, $m$ generally is small [7]. For 6111-T4, $m$ is negative [11]. And the introduction of $m$ will make the model much more complex. Actually, the development of constitutive equation for plastic flow in high velocity forming is still in active research [53-56]. So the effect of strain rate sensitivity will not be considered in this chapter.

Rectangular cup is a common stamping product [112-114]. We will start with rectangular cup forming to theoretical analysis. Figure 6.28 shows the plan view of a quarter of a rectangular cup [112]. $B_{1}, B_{2}$ and $P_{1}, P_{2}$ are half of the blank and punch sizes respectively, $c_{1}, c_{2}$ are the clearance between the punch and the die, and $b_{1}, b_{2}$ are the length of the blank under the binder in the two directions. The subscripts 1 and 2 denote 
the dimensions in the $\mathrm{x}$ and $\mathrm{y}$ directions respectively. The point $\mathrm{C}$ is the center of the rectangular cup.

Apparently, the rectangular cup can be seen as three regions. $A_{1}$ and $A_{2}$ are the regions of the straight sides, and have similar deformation mode. The region OABD is the corner of the part. Generally, this region is considered as an axisymmetric cup, which is different from $A_{1}$ and $A_{2}$. Here we use region OAD to represent the axisymmetric cup. Below we will discuss the deformation modes at the straight side and the corner region respectively.

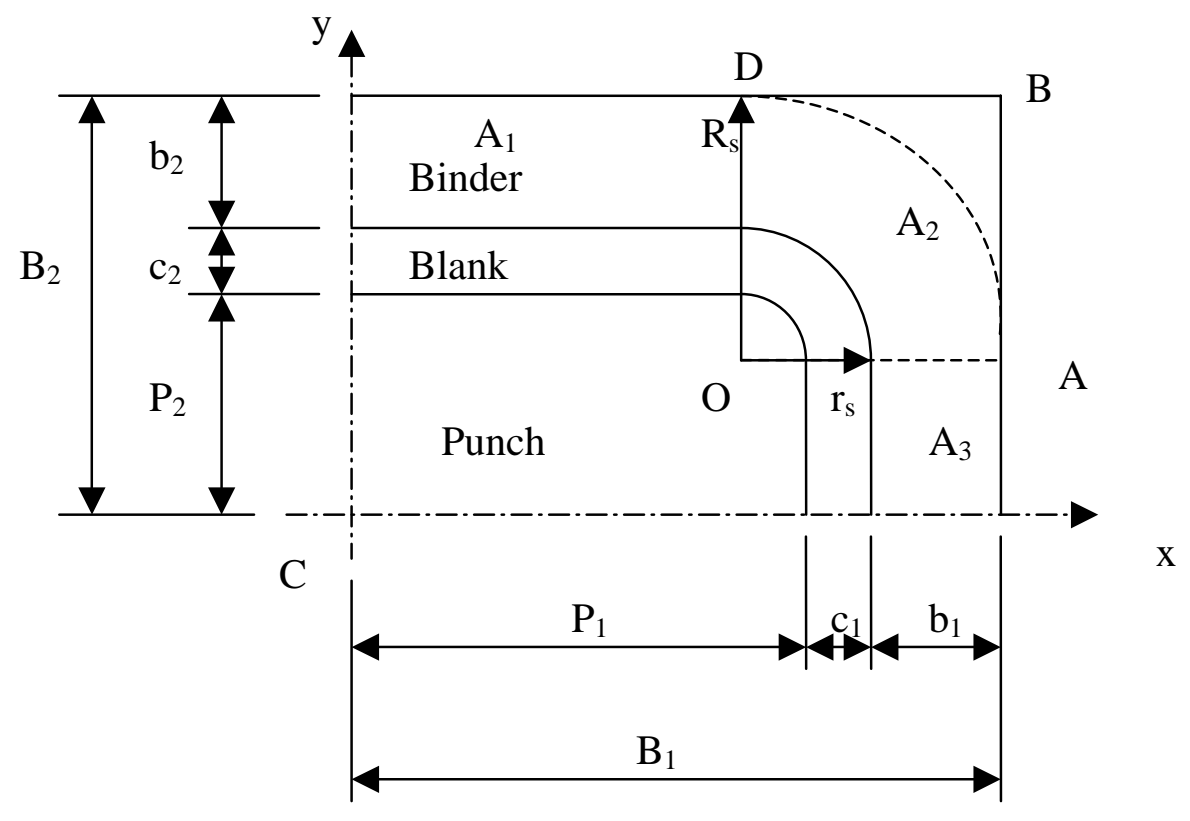

Figure 6.28 Plan view of a quarter of a box [112] 


\subsubsection{Straight side analysis}

\subsubsection{Conventional stamping}

To simplify the analysis, we assume the process is in the plane strain state. And since it is the sheet forming, the process is also in the plane stress state. So the strain and stress states are

$\varepsilon_{1}=-\varepsilon_{3} ; \quad \varepsilon_{2}=0$

And $\sigma_{1}=2 \sigma_{2} ; \quad \sigma_{3}=0$

Therefore, $\bar{\varepsilon}=\frac{2}{\sqrt{3}} \varepsilon_{1}$

$$
\begin{aligned}
\bar{\sigma} & =\frac{\sqrt{3}}{2} \sigma_{1} \\
\sigma_{1} & =K\left(\frac{2}{\sqrt{3}}\right)^{n+1} \varepsilon_{1}^{n}
\end{aligned}
$$

The principal direction, 1 , is in the sectioning plane and the direction 2 is perpendicular to this. The major principal tension at a point [88], $\mathrm{T}_{1}$, in the sectioning plane is

$T_{1}=\sigma_{1} t=K t_{0}\left(\frac{2}{\sqrt{3}}\right)^{n+1} \varepsilon_{1}^{n} \exp \left(-\varepsilon_{1}\right)$

Clearly when the tension reaches a maximum at a point, the sheet will continue to deform at that point under a failing tension. Other regions of the sheet will unload elastically and 
the sheet will fail at that point where the tension maximum occurred [88]. The tension reaches a maximum at a strain of

$$
\varepsilon_{1}^{*}=n
$$

For the deformation of the straight side shown in Figure 6.29 [88, 112], from the center C to the point of tangency G, the sheet is stretching and sliding outwards against friction and the friction force on the sheet acts towards C. From the point of contact with the die $\mathrm{H}$ to the outer edge, the sheet is sliding inwards and the friction force on the sheet acts outwards [88].

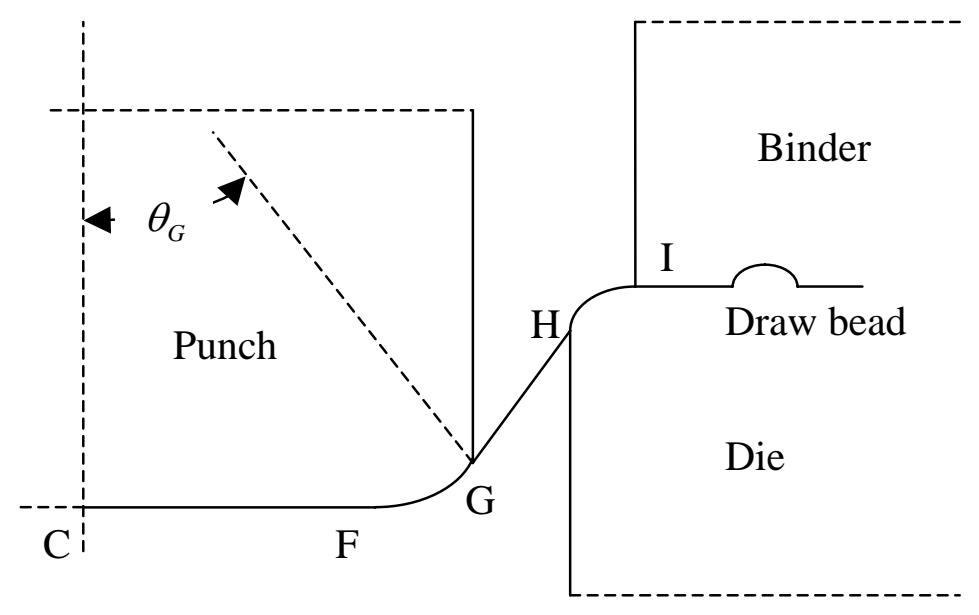

Figure 6.29 Half-section of straight side of a partially drawn box by conventional stamping [88,112] 
We can find the relationships among the tensions of the different positions. The tension at point $\mathrm{G}$ can be given as

$T_{1 G}=T_{1 C} \exp \left(\mu \theta_{G}\right)$

where $\theta_{G}$ is the wrap angle and can be determined from the punch depth $h$ and the tool geometry. If the punch face is only gently curved, the tension will only increase slowly with distance from $\mathrm{C}$ to $\mathrm{F}$. At the corner radius, the tension increases rapidly from $\mathrm{F}$ to $\mathrm{G}$.

In the side wall, the sheet is not in contact with the tooling and the tension is constant, i.e. $T_{1 G}=T_{1 H}$. From $\mathrm{H}$ to $\mathrm{I}$, the tension falls to $T_{1 I}=T_{1 H} \exp \left(-\mu \theta_{G}\right)$. Because of the draw beads, the tension at the flange can be given as $T_{1 I}=2 \mu B_{c}+\Delta T_{d}$, where $B_{c}$ is the clamp load and $\Delta T_{d}$ is the tension increase due to the draw beads. If the strain of point $\mathrm{C}$ is given, we can calculate the tension of this point according to Eq. (6.11), and then determine the whole tension distribution over the section [88].

It can be seen clearly that the bottom region has the low tension and low strain. The high tension and high strain are at the side wall. Generally, the tearing failure doesn't happen at the straight side in conventional stamping.

Above analysis gives a 2D model of stamping. But it is still worth to do such analysis since 2D model is simple [88]. The 2D analysis of conventional stamping here can be applied to compare with next 2D analysis of EM assisted stamping. In this way, a better understand of EM assisted stamping can be achieved. 


\subsubsection{EM assisted stamping}

The difference between EM assisted stamping and conventional stamping is the application of the EM forces, which will make the "bubble" at the bottom region. Figure 6.30 is the schematic half section of the straight side after an electromagnetic pulse. The area just below the EM coil at the bottom region is pushed up and stretched to a "bubble". The stretched "bubble" provides extra line length and leads the force relaxation. Therefore, when the punch advances, it will get small resistance force until the "bubble" is pulled flat. Before the "bubble" is pulled flat, there is no tension from C to K. And the tension from $\mathrm{M}$ and $\mathrm{F}$ is small to pull the "bubble" flat.

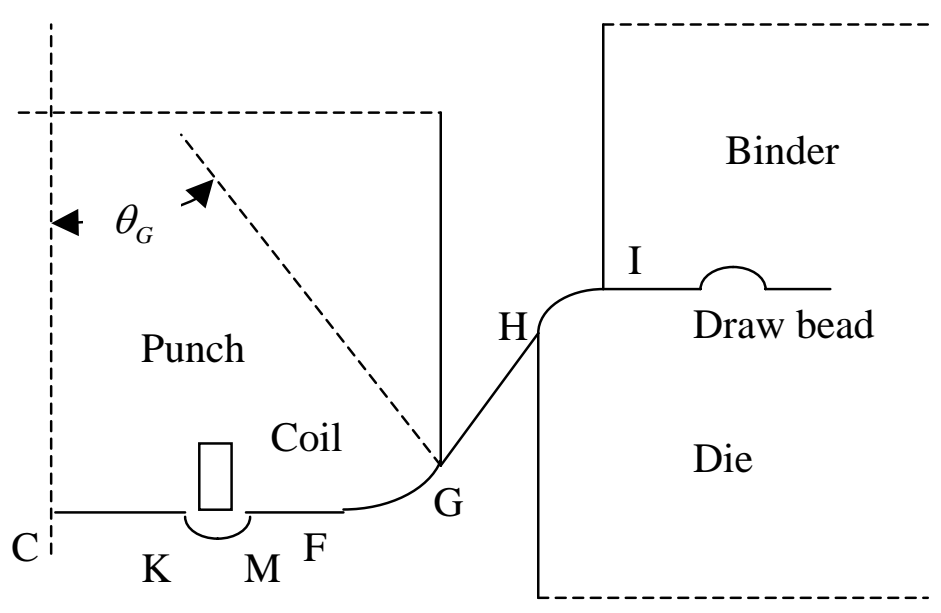

Figure 6.30 Schematic half-section of straight side of a partially drawn box by EM assisted stamping 
Assuming the length between $\mathrm{K}$ and $\mathrm{M}$ before stretching is $l$. After the stretching, the length becomes $L$. So the extension from $\mathrm{K}$ and $\mathrm{M}$ is $\Delta l=L-l$. Also, the EM stretching only causes the elongation in the sectioning plane, i.e. in the plane strain state, and we assume the stretching is uniform along $\mathrm{K}$ to $\mathrm{M}$. So there are

$$
\varepsilon_{1}^{e}=-\varepsilon_{3}^{e}=\ln \left(\frac{L}{l}\right) \quad \text { and } \quad \varepsilon_{2}^{e}=0
$$

Because of the EM pulse, the strains are different along C to F. The strain at K-M becomes $\left(\varepsilon_{1}+\varepsilon_{1}^{e}\right)$. The strains at C-K and M-F are still $\varepsilon_{1}$. Due to strain hardening, the metals from $\mathrm{K}$ to $\mathrm{M}$ are harder than the rest of this region.

After the EM pulse, the punch will advance $\Delta h$ to pull the "K-M bubble" flat, where $\Delta h=\Delta l \sin \theta_{G}$. Since K-M becomes flat, there will be tension from $\mathrm{C}$ to $\mathrm{K}$. With the advance of the punch, the metals at C-K and M-F will stretch first because the metals of K-M are harder. After the metals at C-K and M-F reach the strain $\left(\varepsilon_{1}+\varepsilon_{1}^{e}\right)$, the whole bottom region will stretch together. The value of tension is determined by the areas without EM forming since EM forming will harden the EM stretched area.

Above analysis shows that the draw depth can increase by $\Delta h=\Delta l \sin \theta_{G}$ because of the EM pulse. That improvement is from EM stretching. How about the EM draw-in from the flange? Because the flange of straight side does not involve the flange shrink, there is almost no increase of resistance force for the flange draw in, i.e. $T_{1 I}=2 \mu B_{c}+\Delta T_{d}$. So there will be more draw in from the straight side flange with the punch advance. 
We should notice that there was tearing failure at the bottom region. The strain measurements show the strains near tearing are near the forming limits. So to prevent the tearing, the electromagnetic pulses should not produce too much stretching. The stretching of EM pulses only happens at the area just below the EM coil. It is better to adjust the EM pulse and punch advance procedure to move the stretched area away from the coil position. In this way, the bottom region can have uniform strain distribution and avoid the large stretching.

Above two sections show that the strain states at the straight sides in both conventional and EM assisted stamping are similar and have no dramatic changes. The strain states are still bi-axial stretching at the bottom region. The strain states in the side wall vary from bi-axial stretching to plane strain state. EM pulses increased the amount of stretching and draw-in from the flange at the straight sides.

\subsubsection{Corner side analysis}

\subsubsection{Conventional stamping}

The deformation mode in corner side is different from the one in straight side. The bottom region is in bi-axial stretching. The side wall is in plane strain state. And the flange involves the shrink deformation, which is in tension-and-compression state. The shrink deformation increases the resistance forces for draw in of flange with the advance of the punch. To analyze the corner side, generally it is considered as axisymmetric cup, as the region OAD shown in Figure 6.28 [88, 112-113]. 
In conventional stamping, the tearing happens at the side wall near punch nose, around the position P. Because of the constraining of the punch, this area is in the plane strain state, $d \varepsilon_{\theta}=0$. Since little metals is stretched from the bottom region to the side wall, the hoop strain at this area is considered as $\varepsilon_{\theta}=0$.

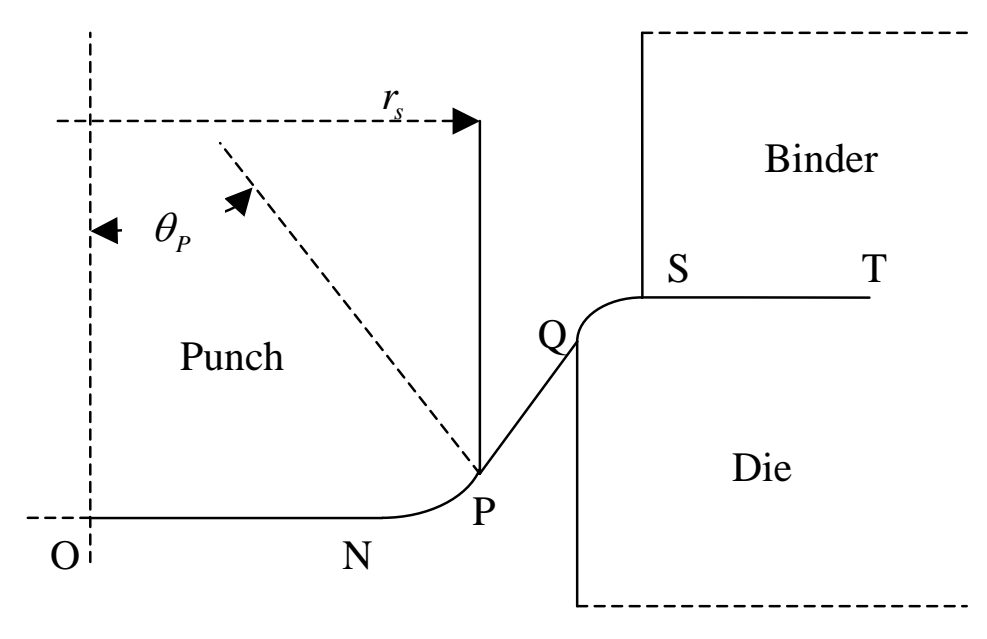

Figure 6.31 Schematic corner side of a partially drawn box by conventional stamping

And the sheet is in the plain stress state condition, $\sigma_{z}=0$. So the effective strain at $\mathrm{P}$ can be expressed as

$$
\bar{\varepsilon}=\frac{1+\bar{R}}{\sqrt{1+2 \bar{R}}} \varepsilon_{r}
$$

And the radial stress at $\mathrm{P}$ can be expressed as 


$$
\sigma_{r}=\frac{1+\bar{R}}{\sqrt{1+2 \bar{R}}} \bar{\sigma}=K\left(\frac{1+\bar{R}}{\sqrt{1+2 \bar{R}}}\right)^{1+n}\left(\varepsilon_{r}\right)^{n}
$$

Since $\varepsilon_{r}=-\varepsilon_{t}$, the thickness is expressed as

$$
t=t_{0} e^{-\varepsilon_{r}}
$$

So the tension at $\mathrm{P}$ is given as $\quad T=\sigma_{r} t=\sigma_{r} \cdot t_{0} e^{-\varepsilon_{r}}$

When $d T=0$, the instability happens. According to the classic analysis $[88,112]$, the critical radial strain is given as

$$
\varepsilon_{r}=n
$$

The maximum tension at the side wall of the conventional stamping is expressed as

$$
T_{\max }=t_{0} K\left(\frac{1+\bar{R}}{\sqrt{1+2 \bar{R}}}\right)^{1+n}\left(\frac{n}{e}\right)^{n}
$$

If the tension at the side wall is bigger than above value, the tearing will happen.

\subsubsection{EM assisted stamping}

During EM assisted stamping, the EM pulse pushes the metals above the coil up and stretches to a "bubble". When the punch goes down, the "bubble" will be pulled flat. This is similar as the one in the straight side. But it has different strain states because of the corner geometry. We will discuss below in detail. 


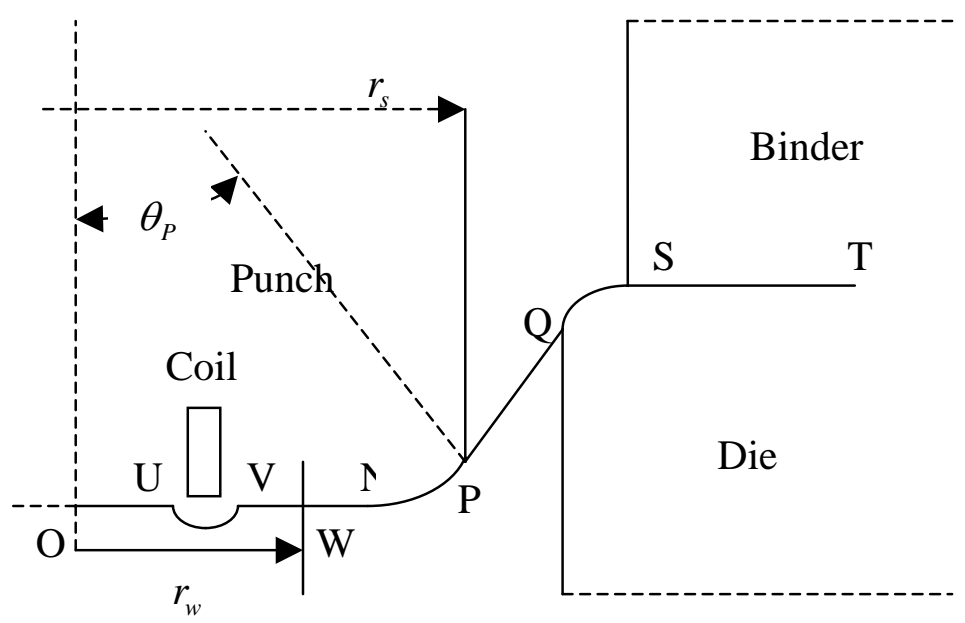

Figure 6.32 Schematic corner side of a partially drawn box by EM assisted stamping

Stretching the "bubble" When the punch advances to a position, an EM pulse is delivered to form a "bubble" just below the coil, as U-V in Figure 6.32. Assuming the distance between $\mathrm{U}$ and $\mathrm{V}$ before EM stretching is $r_{u v}$. After the EM stretching, the line length from $\mathrm{U}$ and $\mathrm{V}$ becomes $\left(\Delta r^{e}+r_{u v}\right)$. Assuming the stretching is uniform from $\mathrm{U}$ and $\mathrm{V}$, the radial strain due to EM pulse is given as $\varepsilon_{r}^{e}=\ln \left(1+\frac{\Delta r^{e}}{r_{u v}}\right)$. And we can assume that there is no hoop strain change during the EM stretching, i.e. $d \varepsilon_{\theta}=0$.

Pulling out the "bubble" Punch continues to advance and then the "bubble" is pulled out. During this process, there is no tension from $\mathrm{O}$ to $\mathrm{U}$ because of this "bubble”. And there is tension from $\mathrm{U}$ to $\mathrm{Q}$ which is needed to pull flat the "bubble” and to increase the 
hoop strain. The strain states from $\mathrm{U}$ to $\mathrm{P}$ are bi-axial stretching. At the area of the side wall near punch nose, the strain state becomes bi-axial because of the metals stretched from the bottom region. At the rest of the side wall, the strain states are still close to plane strain state. Because of the "bubble", the tension at the side wall should not be large, which means there should be no draw in from the flange during this process.

After "Bubble" pulling out The punch will advance a displacement of $\Delta h$ to pull the "bubble" flat, where $\Delta h=\Delta r^{e} \sin \theta_{p}$. After the "bubble" is pulled flat, the tension appears on the whole bottom region. The strain states of the bottom region are still biaxial stretching. Due to EM stretching, the metals at U-V have extra strain $\left(\varepsilon_{r}^{e}\right)$, which makes this area harder than the rest of bottom region due to strain hardening. Therefore, the metals of U-V do not involve plastic deformation until the rest of bottom region are stretched to the same effective strain. At the side wall, the metals begin to deform in plane strain state, i.e. $d \varepsilon_{\theta}=0$. And the metals are drawn in from the flange again.

\subsubsection{Stretching and Draw-in of EM assisted stamping}

Last section shows that the strain states at the bottom region and some region of the side wall are biaxial stretching. From FLD, the equi-biaxial strain state has higher forming limits than the other strain states in the right side of FLD. So it is better to adjust the EM pulse and punch advance to make above strain states equi-biaxial stretching. Then more metal can be stretched from the bottom region to the side wall and becomes part of side wall, which will lead the higher draw depth. 
Besides the stretching of the bottom region, there is another contribution to the improvement of draw depth: draw-in from the flange. The experiments show that the draw-in increased in EM assisted stamping, comparing to the conventional stamping. More draw-in from the straight flange is not difficult to explain, since the resistance force doesn't increase. While more draw-in from the corner side means higher tension in EM assisted stamping than the maximum tension in the conventional stamping. Is it caused by the improvement of force-bearing ability in side wall of corner, or other reasons?

The biggest difference at the side wall of corner between the conventional stamping and the EM assisted stamping is the strain state at the side wall near punch nose. In the conventional stamping, this place is in plane strain state. This state has been discussed in last section. While in the EM assisted stamping, this place is in plane strain state with biaxial pre-strain caused by previous conventional stamping.

Assuming the bi-axial pre-strains are $\left(\varepsilon_{r 1}, \varepsilon_{\theta 1}\right)$, and $\varepsilon_{\theta 1}=\rho_{1} \varepsilon_{r 1}$ where $\rho_{1}$ is constant ratio between $\varepsilon_{r 1}$ and $\varepsilon_{\theta 1}$. So the effective strain of the bi-axial pre-strain can be expressed as $\bar{\varepsilon}_{1}=\frac{1+\bar{R}}{\sqrt{1+2 \bar{R}}} \sqrt{1+\rho_{1}^{2}+\frac{2 \bar{R}}{1+\bar{R}} \rho_{1}} \varepsilon_{\theta 1}$

After the "bubble" is pulled flat, the punch nose area begins to deform in plane strain state, i.e. $d \varepsilon_{\theta}=0$. And then we let $\varepsilon_{r 2}=d \varepsilon_{r}$ for the strain incremental during this plane strain deformation. So the effective strain and stress for this plane strain deformation can be given as 


$$
\begin{aligned}
& \bar{\varepsilon}_{2}=\frac{1+\bar{R}}{\sqrt{1+2 \bar{R}}} \varepsilon_{r 2} \\
& \bar{\sigma}_{2}=K\left(\bar{\varepsilon}_{1}+\bar{\varepsilon}_{2}\right)^{n}=K\left(\frac{1+\bar{R}}{\sqrt{1+2 \bar{R}}}\right)^{n}\left(\sqrt{\left.1+\rho_{1}^{2}+\frac{2 \bar{R} \rho_{1}}{1+\bar{R}} \varepsilon_{\theta 1}+\varepsilon_{r 2}\right)^{n}}\right. \\
& \sigma_{r 2}=\frac{1+\bar{R}}{\sqrt{1+2 \bar{R}}} \bar{\sigma}_{2}=K\left(\frac{1+\bar{R}}{\sqrt{1+2 \bar{R}}}\right)^{n+1}\left(\varepsilon_{\theta 1} \sqrt{1+\rho_{1}^{2}+\frac{2 \bar{R} \rho_{1}}{1+\bar{R}}}+\varepsilon_{r 2}\right)^{n}
\end{aligned}
$$

And then the thickness strain is given as $\varepsilon_{t}=\varepsilon_{t 1}+\varepsilon_{t 2}=-\rho_{1} \varepsilon_{\theta 1}-\varepsilon_{\theta 1}-\varepsilon_{r 2}$

So the tension of the side wall of corner for the plane strain deformation with bi-axial pre-strain situation can be given as $T=\sigma_{r 2} t=\sigma_{r 2} t_{0} e^{-\rho_{1} \varepsilon_{\theta 1}-\varepsilon_{\theta 1}-\varepsilon_{r 2}}$

i.e. $T=K\left(\frac{1+\bar{R}}{\sqrt{1+2 \bar{R}}}\right)^{n+1}\left(\varepsilon_{\theta 1} \sqrt{1+\rho_{1}^{2}+\frac{2 \bar{R} \rho_{1}}{1+\bar{R}}}+\varepsilon_{r 2}\right)^{n} t_{0} e^{-\rho_{1} \varepsilon_{\theta 1}-\varepsilon_{\theta 1}-\varepsilon_{r 2}}$

The bi-axial pre-strain has two effects on the side wall. First is to increase the effective stress, and the second one is to reduce the sheet thickness. They have contradictory effects on the final tension of the side wall. We can compare the tension of the side wall of corner in EM assisted stamping with the maximum tension in the conventional stamping, Eq. (6.21), to check if there is the improvement of force-bearing ability in side wall of corner due to the bi-axial pre-strain.

$$
\frac{T}{T_{\max }}=\left(\frac{\varepsilon_{\theta 1}}{n} \sqrt{1+\rho_{1}^{2}+\frac{2 \bar{R} \rho_{1}}{1+\bar{R}}}+\frac{\varepsilon_{r 2}}{n}\right)^{n} e^{n-\rho_{1} \varepsilon_{\theta 1}-\varepsilon_{\theta 1}-\varepsilon_{r 2}}
$$


For 6111-T4, we can take $n=0.245$ and $\bar{R}=0.8$ [56]. We will consider several situations below.

(1) If $\rho_{1}=0$, there is only hoop strain in the pre-strain. Eq. (6.27) becomes

$$
\frac{T}{T_{\max }}=\left(\frac{\varepsilon_{\theta 1}}{n}+\frac{\varepsilon_{r 2}}{n}\right)^{n} e^{n-\varepsilon_{\theta 1}-\varepsilon_{r 2}}
$$

According to above equation, we can make the diagram of the $\frac{T}{T_{\max }} \sim \mathcal{E}_{r 2}$, shown as

Figure 6.33. The diagram shows that for $\rho_{1}=0$ case, $\frac{T}{T_{\max }} \leq 1$.

(2) If $\rho_{1}=0.5$, Eq. (6.27) becomes

$$
\frac{T}{T_{\max }}=\left(\frac{\varepsilon_{\theta 1}}{n} \sqrt{1+\frac{1}{4}+\frac{\bar{R}}{1+\bar{R}}}+\frac{\varepsilon_{r 2}}{n}\right)^{n} e^{n-\varepsilon_{\theta 1}-\varepsilon_{r 2}}
$$

According to above equation, we can make the diagram of the $\frac{T}{T_{\max }} \sim \varepsilon_{r 2}$, shown as

Figure 6.34. The diagram shows that for $\rho_{1}=0.5$ case, $\frac{T}{T_{\max }}<1$.

(3) If $\rho_{1}=1$, there is equi-biaxial stretching in pre-strain. Eq. (6.27) becomes

$$
\frac{T}{T_{\max }}=\left(\frac{\varepsilon_{\theta 1}}{n} \sqrt{2+\frac{2 \bar{R}}{1+\bar{R}}}+\frac{\varepsilon_{r 2}}{n}\right)^{n} e^{n-\varepsilon_{\theta 1}-\varepsilon_{r 2}}
$$


According to above equation, we can make the diagram of the $\frac{T}{T_{\max }} \sim \varepsilon_{r 2}$, shown as

Figure 6.35. The diagram shows that for $\rho_{1}=1$ case, $\frac{T}{T_{\max }}<1$.

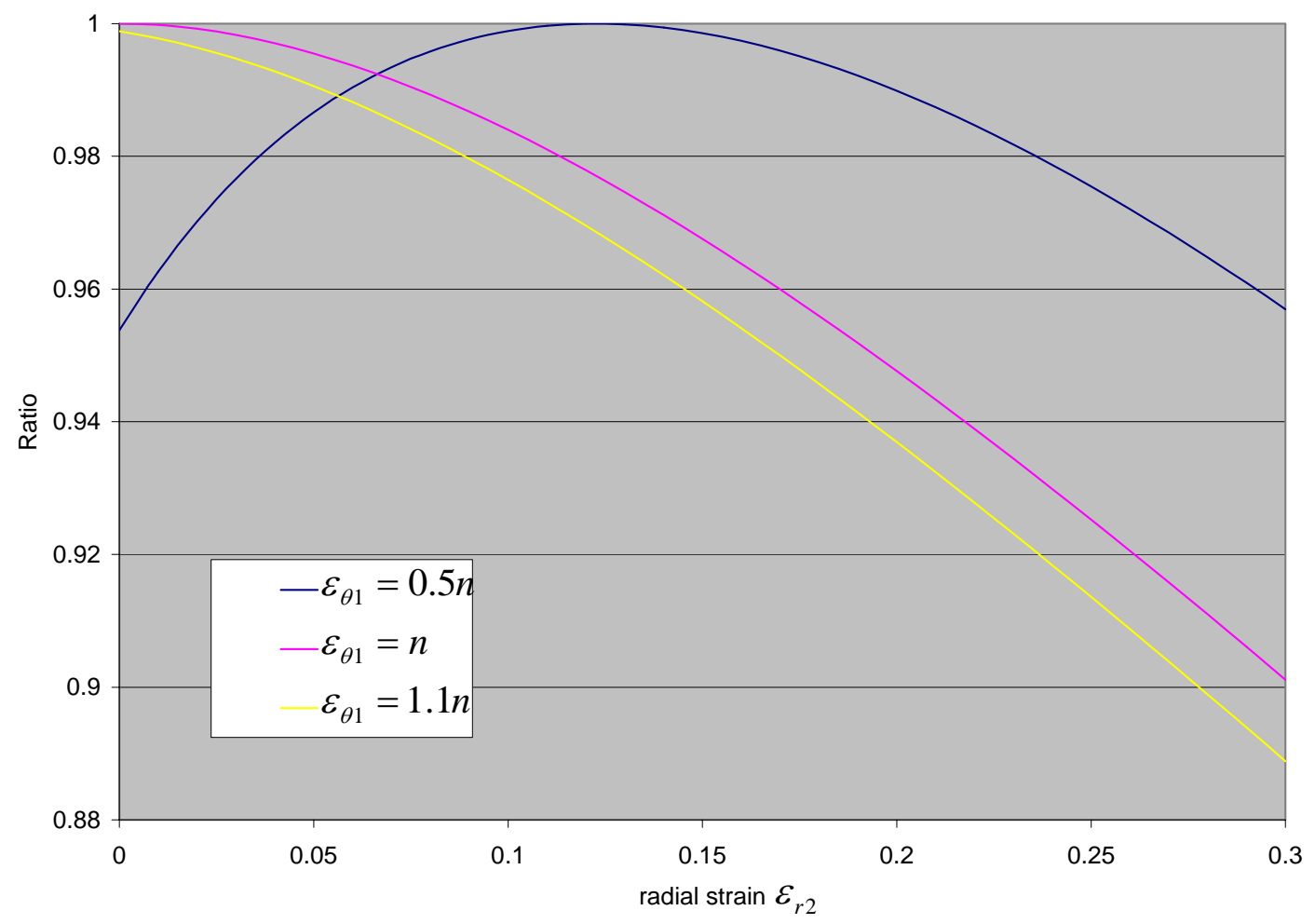

Figure 6.33 Diagram of $T / T_{\max } \sim \varepsilon_{r 2}$ for $\rho_{1}=0$ 


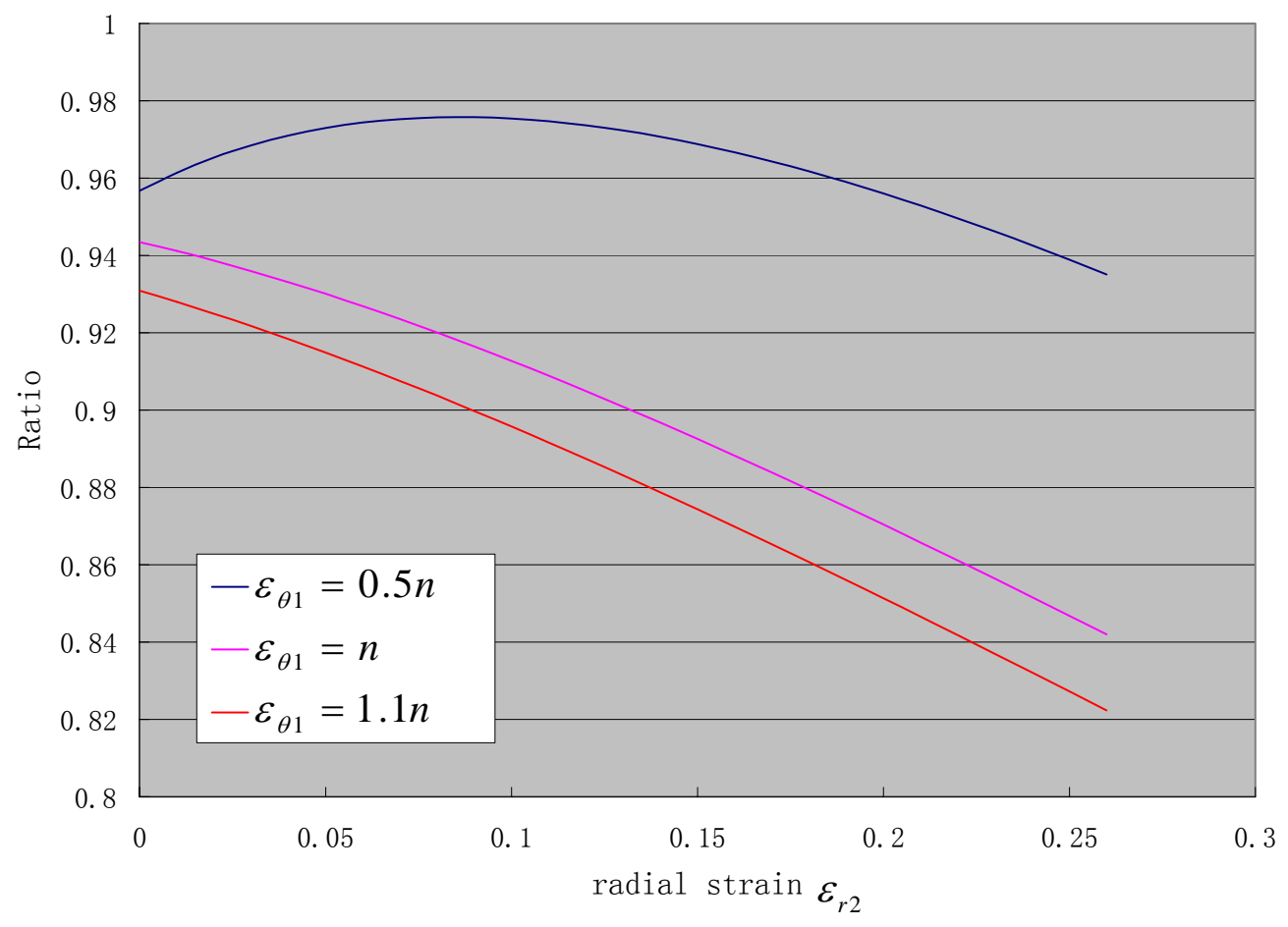

Figure 6.34 Diagram of $T / T_{\max } \sim \varepsilon_{r 2}$ for $\rho_{1}=0.5$ 


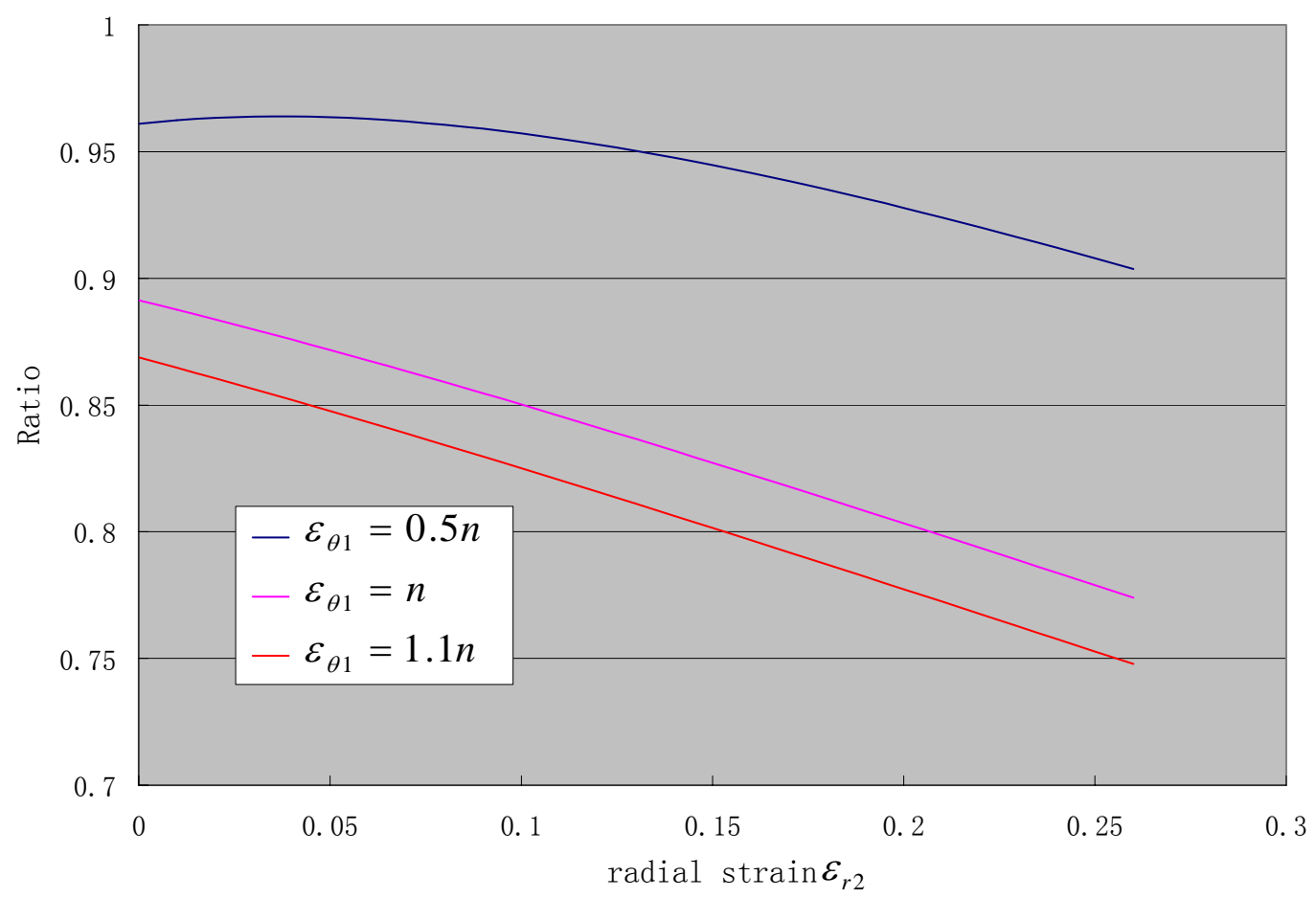

Figure 6.35 Diagram of $T / T_{\max } \sim \varepsilon_{r 2}$ for $\rho_{1}=1$

Above analysis shows that the tension at the side wall of corner in the EM assisted stamping could not be larger than the maximum tension of the side wall in the conventional stamping. So in the EM assisted stamping, the side wall of corner could not provide enough tension to draw more metals in from the corner flange. The other evidence is that the peak punch load did not increase in the EM assisted stamping.

But in experiments, more metal was drawn in from the corner flange in EM assisted stamping. What's the reason for it? It must be relative to the repulsive high velocity EM forming. We will discuss this in next section. 


\subsubsection{EM pulses in EM assisted stamping}

The EM energy level delivered in EM assisted stamping was 5.4kJ. A typical current trace in the EM coil was measured, shown in Figure 6.36.

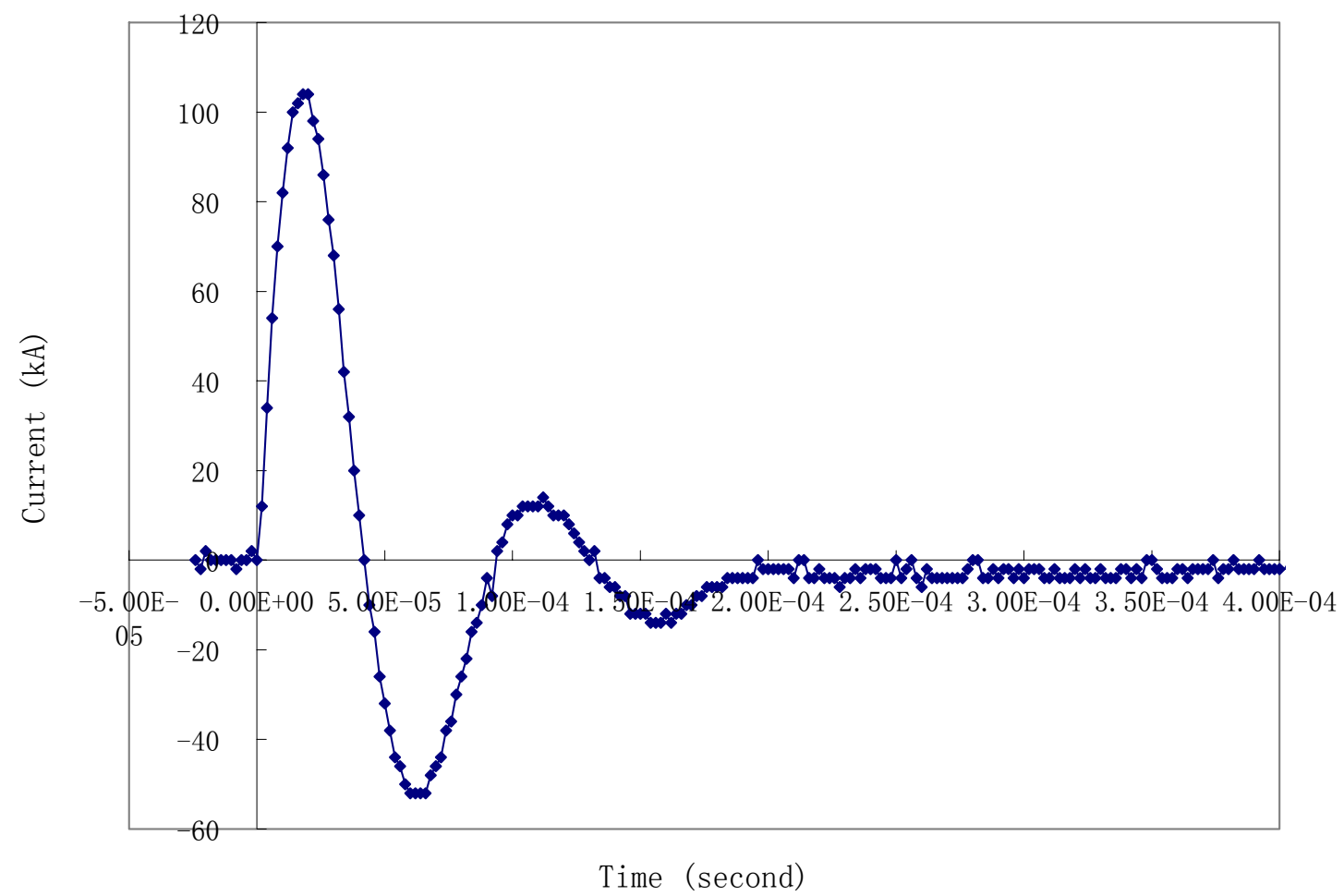

Figure 6.36 A typical current trace for 5.4kJ in EM coil

The peak current was $104 \mathrm{kA}$ and the rising time was $19 \mu \mathrm{s}$. And the coil is $6.29 \mathrm{~mm}$ wide and 1.2m long. From the Figure 6.27, the biggest distance between the coil and the blank was $4.7 \mathrm{~mm}$. And we assume the induced current was $70 \%$ of the primary current. 
According to the equation $[6,59]$ below, the EM pressure between the coil and the blank can be estimated as

$P_{e}=\frac{\mu_{0} I_{1}{ }^{\max } I_{2}{ }^{\max }}{2 \pi d w_{c}}$

From Eq. (6.31), we can estimate the EM pressure is $P_{e}=51 M P a$. And the EM force on the blank is given as

$F_{e}=P_{e} l_{\text {coil }} w_{\text {coil }}=385 \mathrm{kN}$

While the peak punch loads in the conventional and EM assisted stamping were around $190 \mathrm{kN}$, the EM force on the bottom of the metal part was in the similar level to the peak punch load. Eq. (6.31) is the estimate for the EM pressure. To calculate the accurate EM forces, FEM method must be applied, which are beyond this dissertation. Although above calculated EM force must have errors, it still can show that there was large EM force applied on the blank during the EM pulse. This large EM force pushed the blank away from the coil, stretched the blank and then possibly could provide enough forces to draw metals in from the flange.

EM pulses could provide forces to draw metals from the corner flange. But could the side wall of corner provide enough strength to transmit the tension force? Since the EM force reached the peak value in very short time $(19 \mu \mathrm{s})$, the deformation caused by EM force must also be finished in such short time. The high deformation rate must make the blank deformation modes different from the conventional stamping, which is in the quasi-static 
deformation rate. Since the strain rate sensitivity of Al 6111-T4 is negative, we don't consider the strain rate hardening here. So the high rate forming caused by EM pulses must cause a smaller drawing force for the corner flange. The possible reason may be related to the fiction changes in high velocity forming, or strain rate softening, which is beyond this dissertation and will not be discussed here.

Therefore, the EM forces served two functions. One is to stretch the bottom region. The other one is to provide tension force to draw metal from the flange into die cavity. But the function of EM force to draw metals has its working window. For the high clamp load, it could not draw the metals in. To apply EM force, a suitable clamp load should be chosen.

\subsection{Design methodology}

The electromagnetic forces can be applied to stretch the bottom region of the metal part, in order to improve the formability of the stamping process. The design procedure for a common stamping is as follows:

(1) Find the optimum process parameters for the stamping process by conventional methods;

(2) According to the punch geometry, design the electromagnetic coil configuration. The basic rule is to try to make the electromagnetic force distribute uniformly;

(3) Design the electromagnetic energy per pulse according to the coil and sheet metal. The energy may be different in pulses in different stamping stages. And it should make a suitable deformation of the bottom region, neither too much nor too little; 
(4) Design the punch advance procedure according to the optimum conventional stamping and the deformation which electromagnetic force makes. The punch advance should be just enough to pull flat the bottom region. The basic rule for the EM energy design and the punch advance design is to try to apply the electromagnetic force to different areas in different electromagnetic pulses;

(5) Check the strain distribution by the analytical or experimental methods, and adjust the necessary tooling or process parameters to avoid the forming limits or to increase the strain. The rule for clamp load and draw beads is to make it possible for electromagnetic force to draw metals from the flange.

There is no simple analytical method for accurate design of the EM coil and forming parameters. To do so, FEM simulations for electromagnetic forming and for stamping are needed.

There are also some limits for the electromagnetically assisted stamping.

(1) The sheet metal should have high conductivity. Metals with low conductivity are not suitable for this method without a diver sheet;

(2) The punch profile radius should not be small. Otherwise, the bottom region could not be stretched into the side wall;

(3) There will be some waviness at the bottom region. This method is not suitable for the part with high flatness requirement for the bottom region.

(4) Insulation is required. The punch has to be modified to insulate and to accommodate the coil. 


\subsection{Conclusion}

The experiments show the feasibility of the application of electromagnetic forces on the bottom region of the stamping part. By stretching the bottom region with electromagnetic force, the draw depth can be dramatically improved. Besides, this method reduces the reliance on the lubrication, and enables the forming of hard-form materials.

The electromagnetically assisted stamping is promising. While, more works is needed to better understand and predict this process. 


\section{CHAPTER 7}

\section{SPRINGBACK REDUCTION}

\subsection{Introduction to springback}

Springback is defined as the deviation between the final shape at the loading stage and the shape after unloading. It is caused by the elastic recovery of bent area after unloading [88]. Since all the materails have a finite modulus of elasticity, springback is inevitable in the sheet metal stamping. And because springback causes the inaccuracy of the part shape, control of springback is very important to get desired shape of parts.

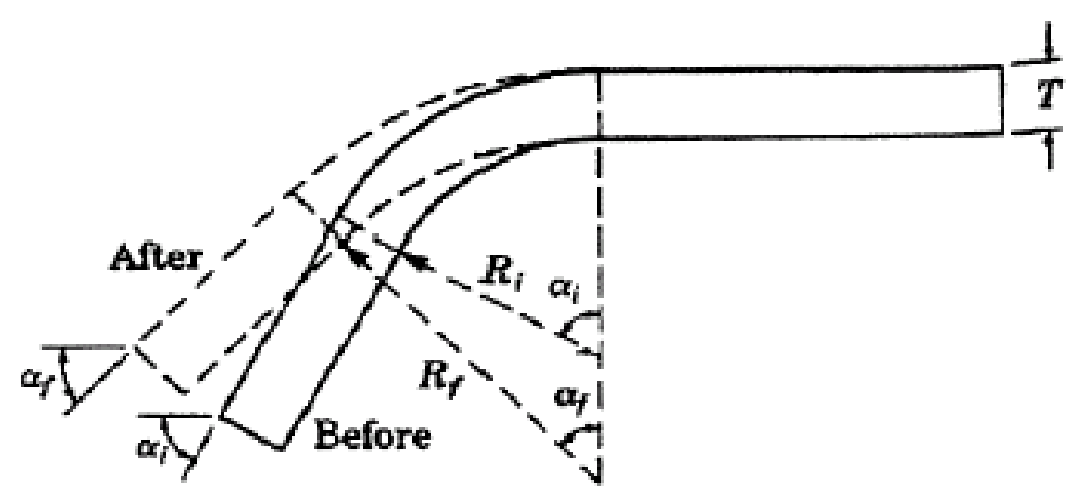

Figure 7.1 Schematic of springback after unloading [88] 
To estimate the springback, the following equation [88] is often used,

$$
\frac{R_{i}}{R_{f}}=4\left(\frac{R_{i} \cdot Y}{E \cdot T}\right)^{3}-3\left(\frac{R_{i} \cdot Y}{E \cdot T}\right)+1
$$

where $R_{i}$ is the bending radius before unloading; $R_{f}$ is the radius after unloading; $\mathrm{T}$ is the thickness of the blank; $\mathrm{Y}$ is the yield strength of the blank; and $\mathrm{E}$ is the elastic modulus of the blank.

The above equation shows that the amount of springback is related to the material properties, and also the tooling geometry. To get the desired shape of stamping parts, tooling designers have to consider the compensation of the sprinback. To correct sprinback, both the material properties and the tooling geometry should be considered [115-119]. It is a very complex process.

Springback is only caused by the elastic recovery of bending area, which is relative small comparing to the whole parts. If a force can be directly applied to this bending area to change the strain distribution of this area, the springback can be changed or even eliminated.

Electromagnetic forming can easily control the spatial distribution and magnitude of the electromagnetic forces. It can produce highly localized deformation. The electromagnetic coil can be put close to the bending area to give the repulsive electromagnetic forces. And the electromagnetic forces can be controlled to give additional deformation at the bending area, which will change the strain distribution of the bending area and hence reduce the 
springback. Therefore, electromagnetic forming is applied to correct the springback of bending in this chapter.

\subsection{Experiment setup}

To demonstrate the feasibility of reducing springback by electromagnetic forming, a single turn coil was built with 0.1875 -inch-radius top. This coil was also used as a 90degree-angle bending die to bend the initially flat blank to 90 degree. A matched 90degree-angle steel female die was used to form the blank.

During the experiment, flat metal blank was positioned between single turn coil and steel female die. By hydraulic equipment, steel female die advanced and bent the metal blank to 90 degree. Then electromagnetic force was applied to the metal blank from the singleturn coil. After the EM forming, the steel female die was removed and the bend angle of blanks was measured to study the springback.

Three different kinds of metals were used, which are listed in Table 7.1. 


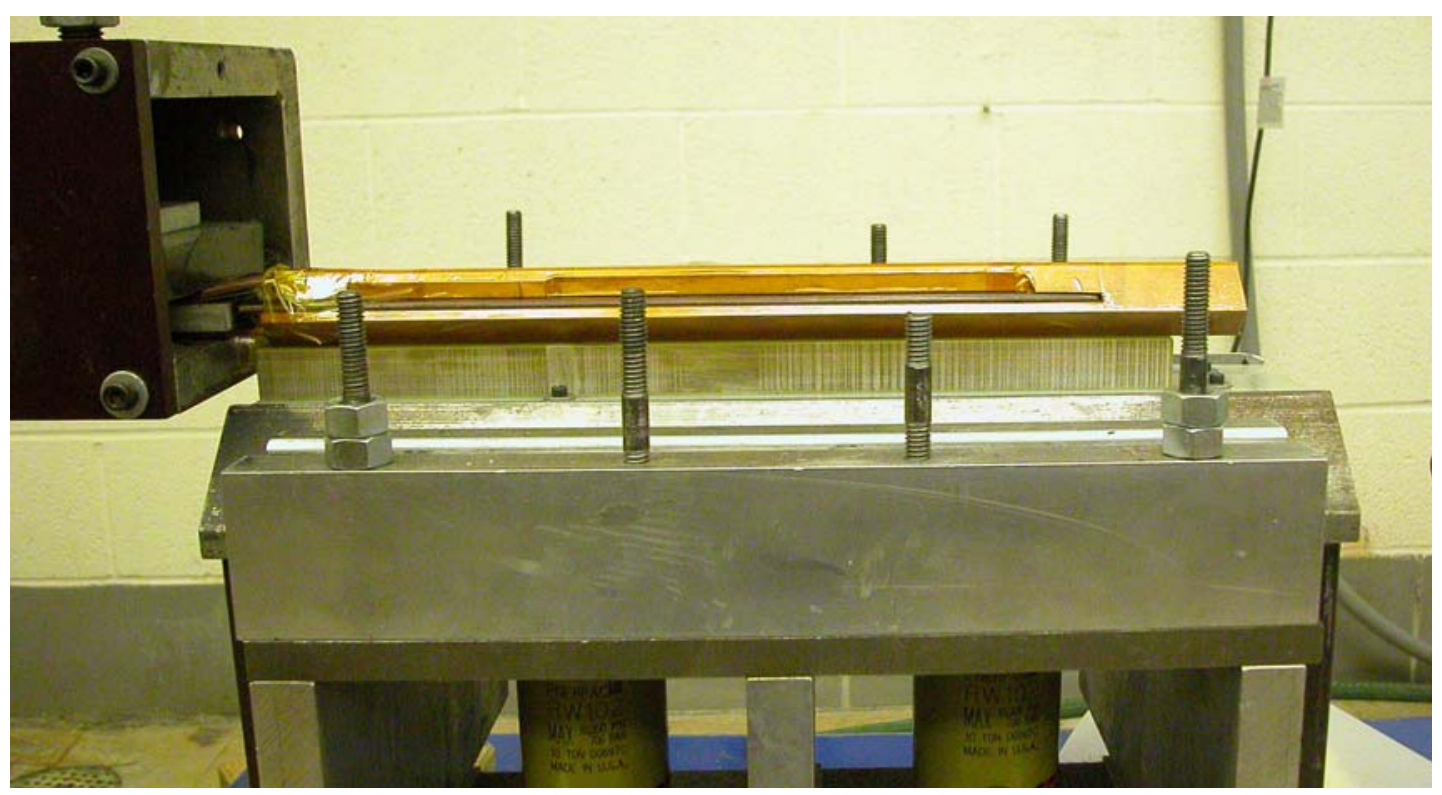

Figure 7.2 Photo of single-turn coil used in experiments

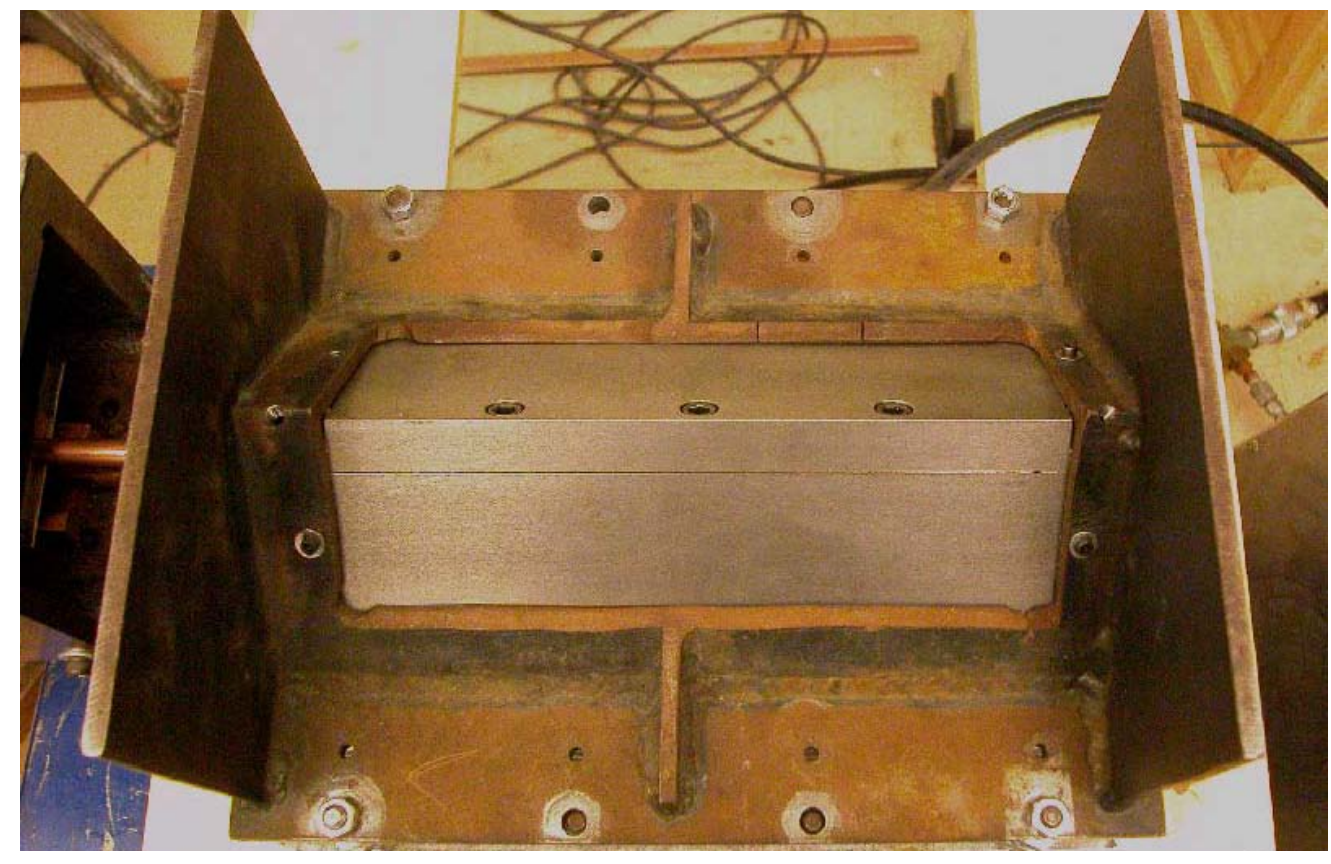

Figure 7.3 Photo of steel female die on the top of single turn coil 


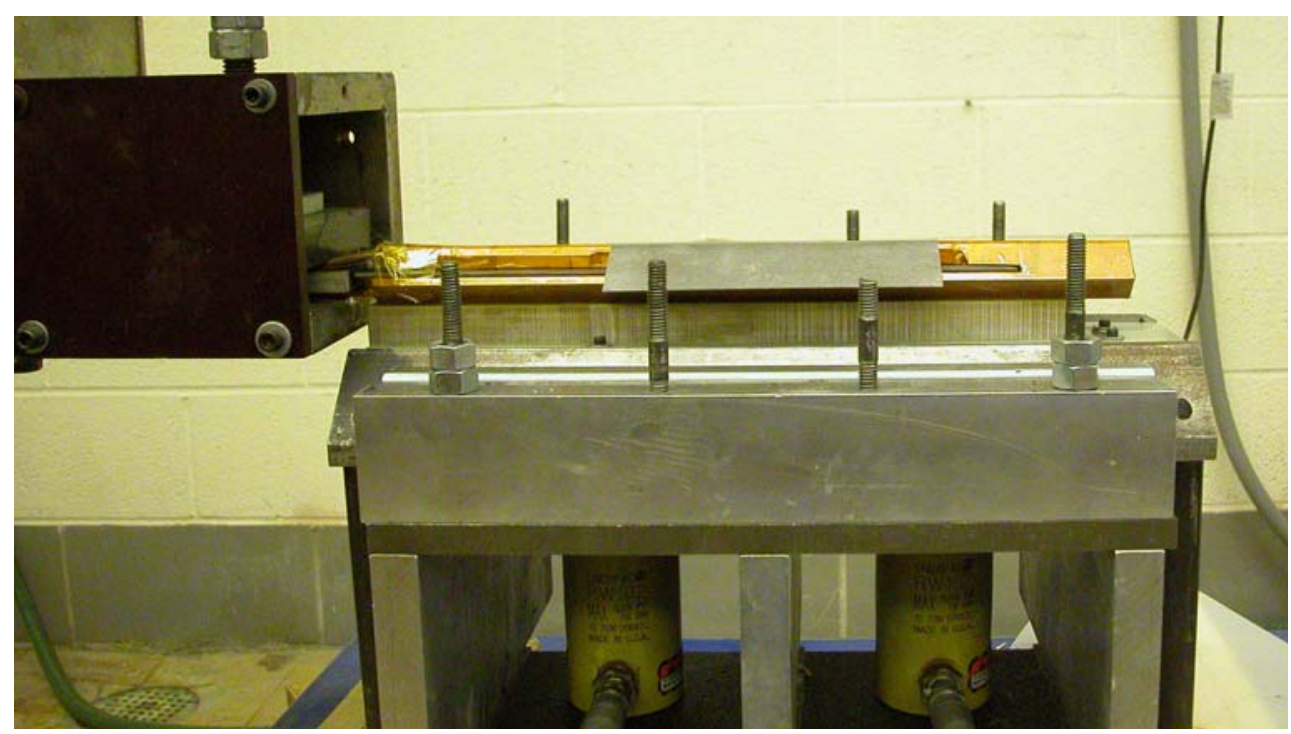

Figure 7.4 Bent metal blank on the top of single turn coil (After bending and electromagnetic forming, the steel die was removed)

Materials $\quad$ Thickness (inch) $\quad$ Yield Stress (MPa) $\quad$ Modulus of Elasticity (Gpa)

\begin{tabular}{cccc}
\hline AA2024-T3 & 0.0200 & 345 & 73.1 \\
\hline Steel, V1 & 0.0150 & 606 & 200 \\
\hline Steel, V16 & 0.0059 & 647 & 200 \\
\hline
\end{tabular}

Table 7.1 Materials Properties

The mechanical and electrical properties of AA2024-T3 are from www.matweb.com [87]. And the mechanical properties of two kinds of steels are from Mala Seth's thesis [120]. 


\subsection{Experiment results}

\subsubsection{AA2024-T3}

Below is the photo of the bent AA2024-T3 blanks after removal from the die. With increase of electromagnetic energy, the springback was reduced and even completely removed at the $4 \mathrm{~kJ}$ of EM energy. When the applied energy was too high, the blank was over bent, i.e. less than 90 degree. The experiment results show the feasibility of reducing springback by electromagnetic forming.
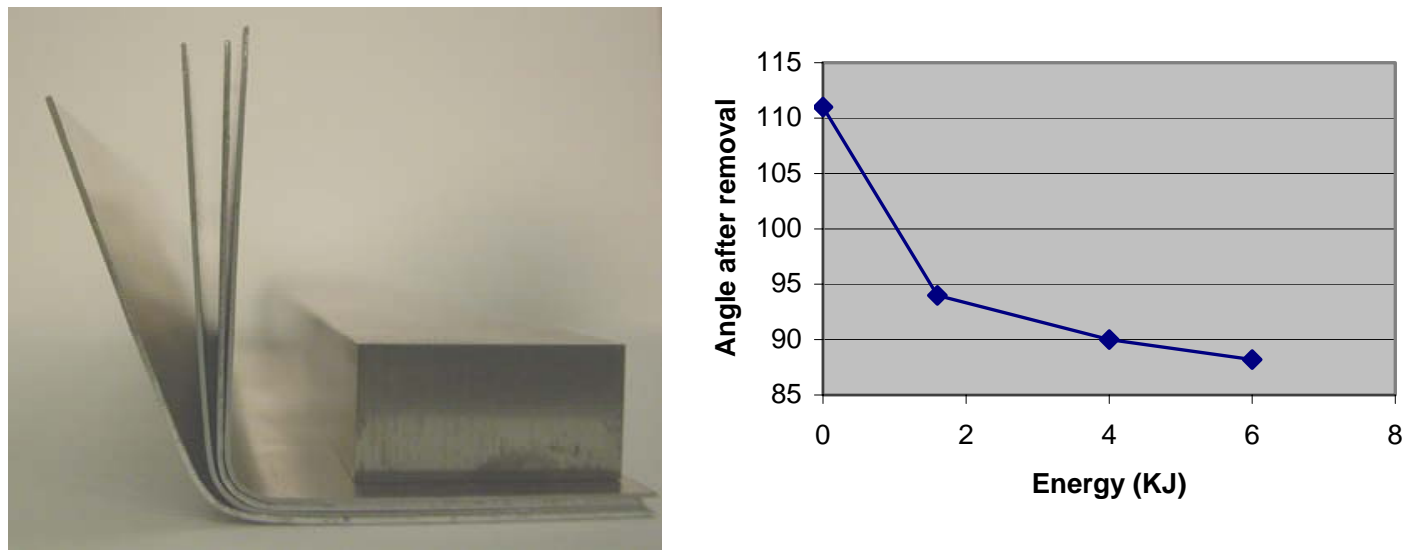

Figure 7.5 Photo of bent AA2024-T3 blanks and the relationship between EM energy and springback angle

\subsubsection{Steel- V1}

Figure 7.6 shows experiment results of steel V1. The springback has been reduced with increase of delivered electromagnetic energy. While, to completely remove the 
springback, more energy was needed. In this experiment, even $12.8 \mathrm{~kJ}$ EM energy could not completely remove the springback.
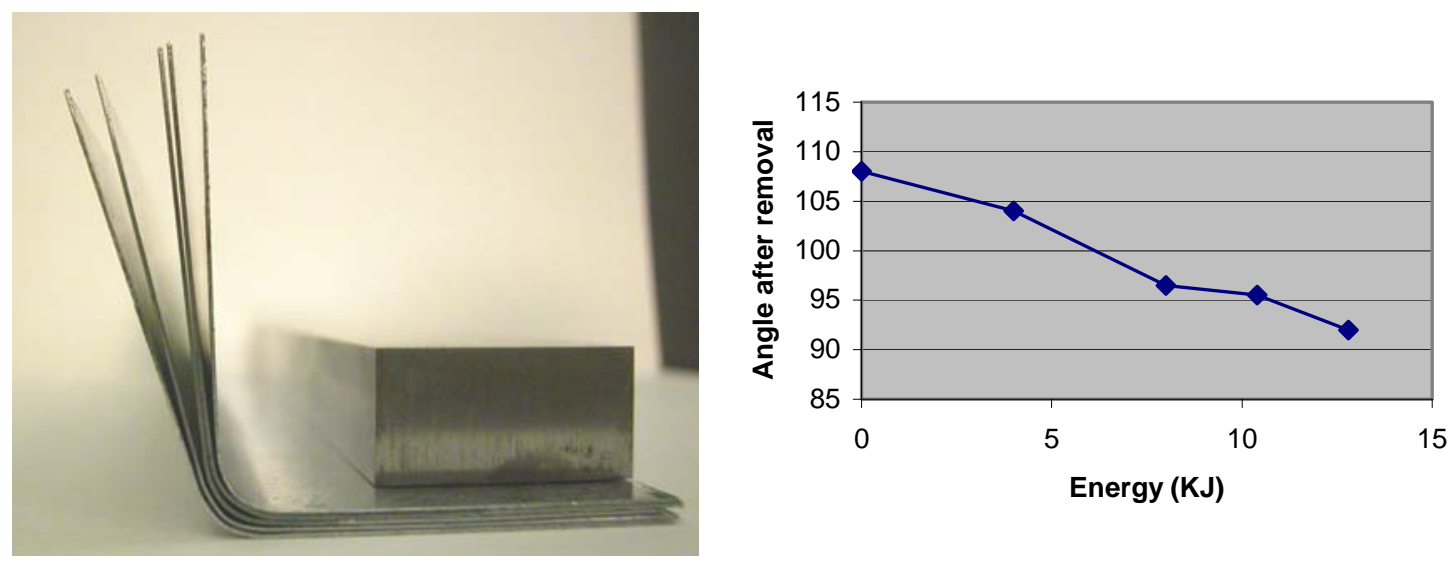

Figure 7.6 Photo of the bent steel-V1 blanks and the relationship between EM energy and springback angle

\subsubsection{Steel -V16}

Figure 7.7 shows experiment results of steel V16. The springback was reduced with increase of delivered electromagnetic energy. At the $8.0 \mathrm{~kJ}$ EM energy, the springback was completely removed. 

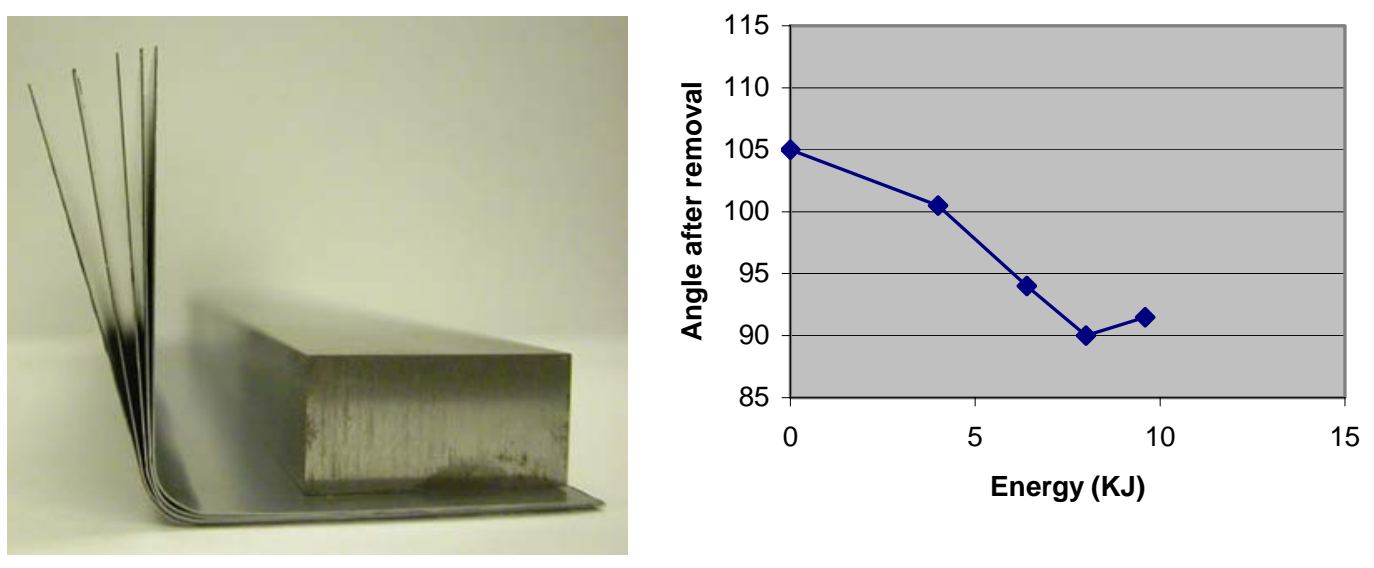

Figure 7.7 Photo of the bent steel-V16 blanks and the relationship between EM energy and springback angle

\subsection{Discussion}

Above experiment show that applying EM forces can reduce and even remove the springback of the bending. To better apply this technology, two important issues should be addressed.

\subsubsection{Materials properties}

The mechanical properties of materials have strong effect on the springback, as mentioned before. While, to reduce the EM forces, the conductivity of metals also is important. The higher the conductivity of the metal is, the bigger the induced current in the metal blank is, and therefore the larger the repulsive EM forces applying on the 
bending area are. To reduce springback of the metal blank with higher conductivity, the smaller EM energy is needed.

AA2024-T3 has higher conductivity than the steels. Although its springback is larger than the steel's, much smaller EM energy $(4 \mathrm{~kJ})$ is needed to completely remove the springback. But for the steel V1, even $12.8 \mathrm{~kJ}$ energy could not remove the springback.

The effect of the electric property can be shown with the skin depth. If the skin depth is larger than the thickness of metal blank, the efficiency of EM forming will be lower. Figure 7.8 is the primary current trace for $8 \mathrm{~kJ}$. From this trace, the rising time is determined as $8.8 \mu$ s. So the frequency of the system is:

$\omega=2 \pi f=\frac{2 \pi}{4 t_{r i \sin g}}=\frac{2 \pi}{4 \times 8.8 \times 10^{-6}}=178 \times 10^{3}$

AA2024-T3 has $5.82 \times 10^{-6}$ ohm-cm electrical receptivity [87]. So the skin depth can be calculated as:

$\delta=\sqrt{\frac{2 \rho}{\mu \omega}}=\sqrt{\frac{2 \times 5.82 \times 10^{-8}}{4 \pi \times 10^{-7} \times 178 \times 10^{3}}}=0.72 \mathrm{~mm}=0.028$ inch

The thickness of AA2024-T3 is 0.02 inch. The skin depth is almost same as the thickness of the metal blank. So the electromagnetic forces could effectively be applied on the blank.

The steel V1 was measured to have $13.06 \times 10^{-6}$ ohm-cm electrical receptivity. So the skin depth can be calculated as: 
$\delta=\sqrt{\frac{2 \rho}{\mu \omega}}=\sqrt{\frac{2 \times 13.06 \times 10^{-8}}{4 \pi \times 10^{-7} \times 178 \times 10^{3}}}=1.08 \mathrm{~mm}=0.0425$ inch

The thickness of steel V1 is 0.015 inch. The skin depth is much bigger than the thickness of the steel blank, which means that electromagnetic forces applied on the blank will fall down [60]. Therefore, EM forming has less efficiency for steel sheets. Despite this, this process still works for steel sheets.

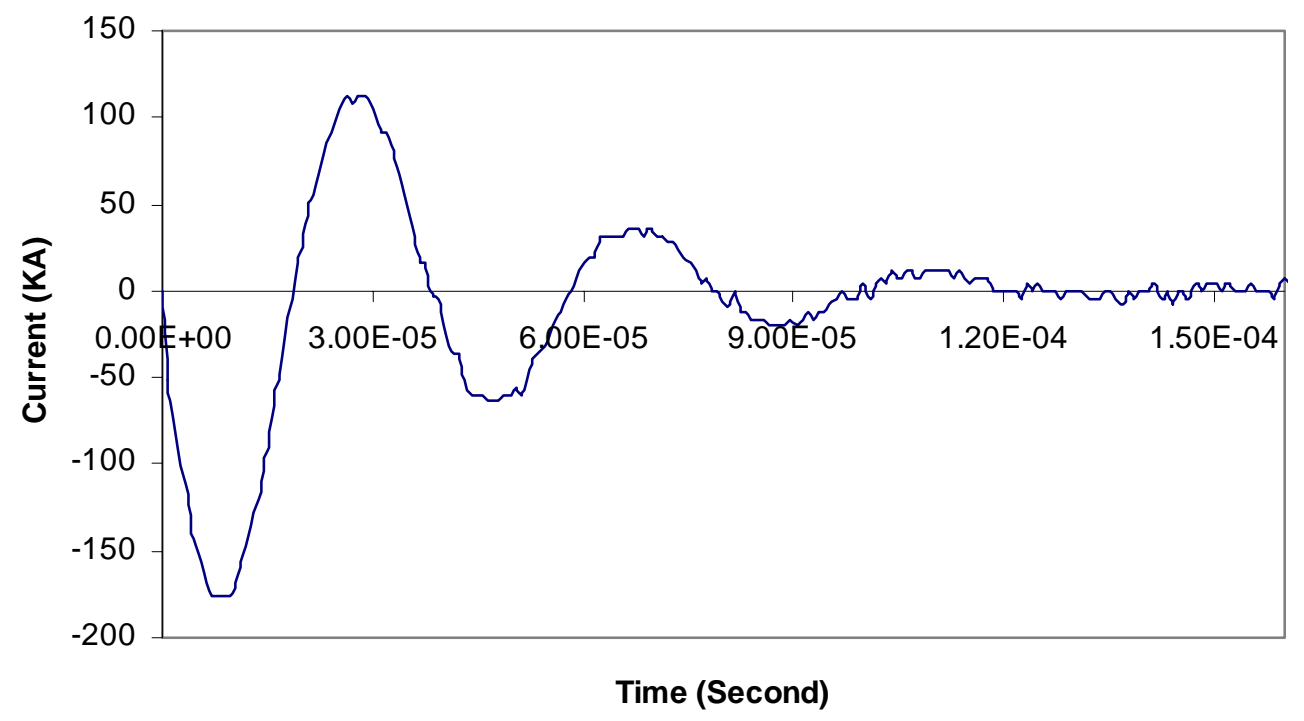

Figure 7.8 Primary current trace at 50\%-8cap (8.0 kJ) 


\subsubsection{EM coil geometry}

EM coil geometry has big effect on the EM forming efficiency. The important issue is to provide the return path for the induced current in the metal blank. If there is no return path for the induced current, the efficiency of the EM forming will be very low.

To investigate the effect of return path, a single turn coil was built, shown as Figure 7.9. This coil has a round bar for return path of primary current. Because the return path of primary current is far away from the metal blank, it is difficult to form return path for the induced current in the metal blank, which will cause low induced current. By coil 2\#, to completely remove springback of AA2024-T3 required $6.4 \mathrm{~kJ}$, which is higher than that of previous coil (4 kJ).

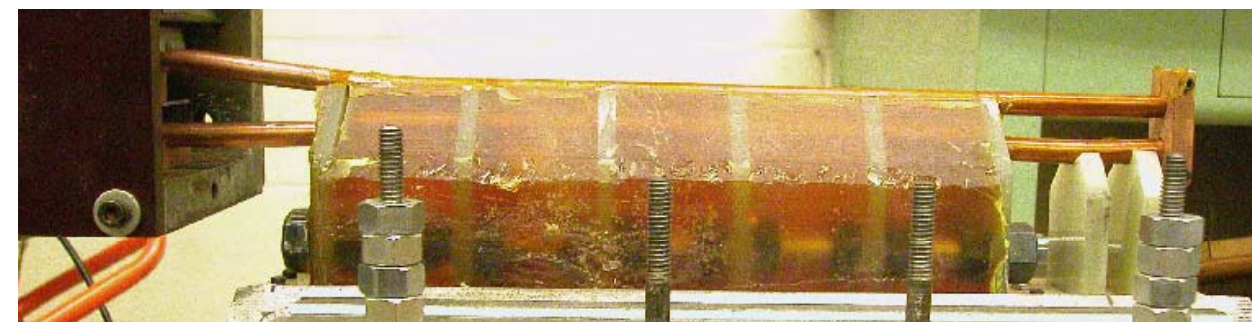

Figure 7.9 Coil configuration 2\# 
(a)

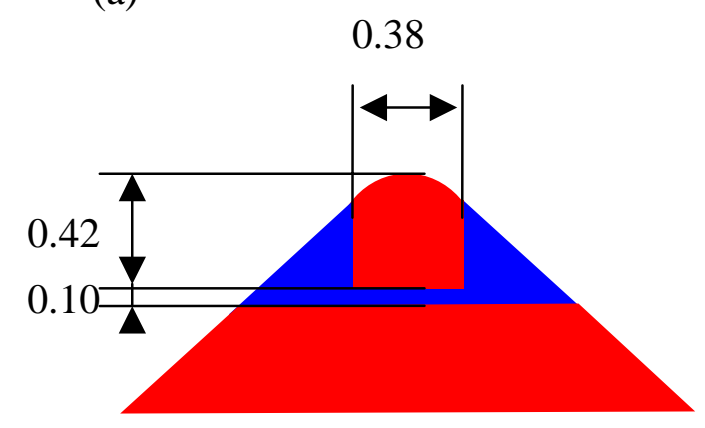

(b)

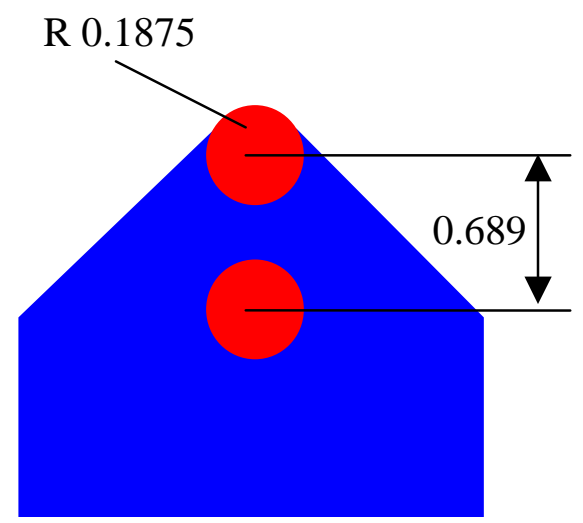

Figure 7.10 Schematic illustration about coil dimensions (a) Coil configuration 1\#; (b) Coil configuration 2\# (unit: inch; Red: EM coil; Blue: Urethane)

\subsection{Conclusion}

Experiment results confirmed that electromagnetic forming is effective method to reduce the springback of bending. It is easy to apply and control. To improve the efficiency of the EM forming, coil configuration and materials conductivity should be considered. 


\section{CHAPTER 8}

\section{CONCLUSION}

This work has carried out electromagnetically assisted sheet metal forming first time. It shows the feasibility of this completely new method. Based on the experiments and analysis, the followings can be concluded:

(1) Electromagnetically assisted sheet metal forming can greatly increase the formability of sheet metal stamping;

(2) For different forming processes, different electromagnetic coils can be easily incorporated into conventional forming tooling;

(3) This method reduces the reliance on the lubrication, which saves the cost of lubricants and the removal;

(4) This method can fabricate the stronger or hard-forming metals, which saves the materials cost because less formable grade metal can be used;

(5) Electromagnetic forces can be applied to reduce springback;

(6) Electromagnetic forces are easy to control, which provides flexible control methods to tune forming process; 


\section{BIBLIOGRAPHY}

1. Glenn S. Daehn, Electromagnetically Assisted Sheet Forming: Enabling Difficult Shapes and Materials by Controlled Energy Distribution, Research Proposal to NSF, (2002)

2. M. L. Bohn, S. G. Xu, K. J. Weinmann, C. C. Chen and A. Chandra, Improving Formability in Sheet Metal Stamping with Active Drawbead technology, Journal of Engineering Materials and Technology, Vol. 123, 4, pp.504-510, (2001)

3. M. Kleiner, M. Geiger, A. Klaus, Manufacturing of Lightweight Components by Metal forming, Annals of the CIRP, 52/2(2003), 521-542

4. Daoming Li, Amit K. Ghosh, Biaxial warm behavior of aluminum sheet alloys, Journal of Materials Processing Technology 145 (2004), 281-293

5. William F. Hosford and John L. Duncan, Sheet metal forming: A review, JOM, Vol. 51, 11, pp. 9-44, (1999)

6. Vincent J. Vohnout, A hybrid Quasi-static/Dynamic Process for Forming Large Sheet Metal Parts From Aluminum Alloys, PhD Dissertation, the Ohio State University, Columbus, Ohio, 1998

7. William F. Hosford and Robert M. Caddell, Metal Forming: Mechanics and Metallurgy, Second Edition, Englewood Cliffs, N.J., Prentice Hall, (1993)

8. Roger Pearce, Sheet Metal Forming, Adam Hilger, Bristol, Philadelphia and New York, (1991)

9. R. H. Wagoner, J.-L. Chenot, Metal forming analysis, Cambridge, New York, Cambridge University Press, (2001)

10. S. S. Hecker, Simple Technique for Determining Forming Limit Curves, Sheet Metal Ind., 52, (1975), 671-676

11. Wonjib Choi, Peter P. Gillis and S. E. Jones, Forming Limit Diagrams, Forming Limit Diagrams: Concepts, Methods, and Applications, Edited by R. H. Wagoner, K. S. Chan, S. P. Keeler, A Publication of TMS, pp.215-237, (1989) 
12. Shiguang Hu and Hezheng Chen, Cold Work Forming Mechanism of Sheet, (in Chinese), Beijing, Defense Industrial Press, (1989)

13. Thomas B. Stoughton, A General Forming Limit Criterion for Sheet Metal Forming, International Journal of Mechanical Science, Vol. 42, pp.1-27, (2000)

14. Hong Yao, Jian Cao, Prediction of Forming Limit Curves Using an Anisotropic Yield Function with Prestrain Induced Backstress, International Journal of Plasticity, Vol. 18, pp.1013-1038, (2002)

15. A. Graf and W. Hosford, Effect of Changing strain paths on Forming Limit Diagrams of Al 2008-T4, Metallurgical and Materials Transactions, Vol. 24A, pp.2503-2511, (1993)

16. Thomas B. Stoughton, Stress-Based Forming Limits in Sheet-Metal Forming, Journal of Engineering Materials and Technology, Vol. 123, October, pp. 417-422, (2001)

17. E. J. Obermeyer, S. A. Majlessi, A review of recent advances in the application of blank-holder force towards improving the forming limits of sheet metal parts, Journal of Materials Processing Technology, 75 (1998), 222-234

18. J. Havranek, Wrinkling limit of tapered press, J. Aust. Inst. Met. 20 (2) (1975), 114119

19. Daoming Li., Amit K. Ghosh and Xiaoxin Xia, Warm forming of aluminum sheet - a review, http://www.mse.engin.umich.edu/research/groups/ghosh/publications/2002

20. D. Banabic, H. J. Bunge, K. Pohlandt., A. E. Tekkaya, Formability of Metallic Materials, Springer- Verlag Berlin Heidelberg, 2000

21. Hua-chu Shin and William R. D. Wilson, Effects of Contact Pressure and Strain on Friction in Sheet-Metal Forming, Tribology Transactions, Vol. 42, 1, pp.144-151, (1999)

22. Jianjun Wei, A. Erdemir and G. Fenske, Dry Lubricant Films for Aluminum Forming, Tribology Transactions, Vol. 43, 3, pp.535-541, (2000)

23. X. Xia and D. T. Llewellyn, Modelling of Stretch Forming Behavior in Steel Strip Using Finite Element Method, Materials Science and Technology, Vol.10, March, pp.232-238, (1994)

24. Claude G. Wall, the Laboratory Evaluation of Sheet Metal Forming Lubricants, Lubrication Engineers, Vol. 40, March, pp.139-147, (1984)

25. Klaus Siegert, Stefan Wagner and Michael Ziegler, Robust Forming Process by Pulsating Blankholder Forces, Multipoint Cushion Systems and Closed Loop Control, 
Sheet Metal Forming Technology, Edited by Mahmoud Y. Demeri, The Minerals, Metals \& Materials Society, pp.1-21, (1999)

26. S. Yossifon, K. Sweeney, M. Ahmetoglu and T. Altan, On the Acceptable Blankholder Force Range in the Deep-drawing Process, Journal of Materials Processing Technology, Vol. 33, pp.175-194, (1992)

27. C.-W. Hsu, A. G. Ulsoy and M. Y. Demeri, An Approach for Modeling Sheet Metal Forming for Process Controller Design, Journal of Manufacturing Science and Engineering, Vol. 122, November, pp.717-724, (2000)

28. John A. Waller, Press Tools and Presswork, the Graphic Group, Burgess Hill, W. Sussex, (1978)

29. Paul B. Schubert, Die Methods: Design, Fabrication, Maintenance, and Application, Book One, Industrial Press Inc., New York, N. Y., (1966)

30. E. Doege and S. Schulte, Design of Deep Drawn Components with Elementary Calculation Methods, Journal of Materials Processing Technology, Vol. 34, pp.439447, (1992)

31. S. Thiruvarudchelvan and W. G. Lewis, Deep Drawing with Blank Holder Force Approximately Proportional to the Punch Force, Journal of Engineering for Industry, Vol. 112, pp.278-285, (1990)

32. J. Cao and M. Boyce, A predictive Tool for Delaying Wrinkling and Tearing Failure in Sheet Metal Forming, Journal of Engineering Materials and Technology, Vol. 119, 4, pp.354-360, (1997)

33. M. A. Ahmetoglu, T. Altan and G. L. Kinzel, Improvement of Part Quality in Stamping by Controlling Blank-holder Force and Pressure, Journal of Materials Processing Technology, Vol. 33, pp.195-214, (1992)

34. Jian Cao, Brad L. Kinsey, Hong Yao, Vikram Viswanathan and Nan Song, Next Generation Stamping Dies-Controllability and Flexibility, Robotics and Computer Integrated Manufacturing, Vol. 7, pp.49-56, (2001)

35. George E. Dieter, Mechanical Metallurgy, Third Edition, McGraw-Hill Book Company, 1986

36. Richard Gedney, Sheet metal formability, Advanced Materials \& Processes, August 2002, 33-36

37. Klaus-Jurgen Pahl, New Developments in Multi-point Die-cushion Technology, Journal of Materials Processing Technology, Vol. 71, pp.168-173, (1997) 
38. Rui Li and Klaus J. Weinmann, Non-Symmetric Panel Forming of AA 6111-T4 Using Active Drawbeads, Sheet Metal Forming Technology, Edited by Mahmoud Y. Demeri, The Minerals, Metals \& Materials Society, pp.39-52, (1999)

39. J. R. Michler, A. R. Kashani, M. L. Bohn and K. J. Weinmann, A Strip Drawing Simulator with Computer Controlled Drawbead Penetration and Blankholder Pressure, Journal of Materials Processing Technology, Vol. 43, pp.177-194, (1994)

40. Hoon Huh and Se-Ho Kim, Optimum Process Design in Sheet-Metal Forming with Finite Element Analysis, Journal of Engineering Materials and Technology, Vol. 123, October, pp.476-481, (2001)

41. R. J. Comstock Jr., Kaiping Li and R. H. Wagoner, Simulation of Axisymmetric Sheet Forming Tests, Journal of Materials Processing Technology, Vol. 117, pp.153-168, (2001)

42. Donald A. Peterson, Dorothy M. Wylo and Frances M. Kania et al, Progressive Dies: Principles and Practices of Design and Construction, Society of Manufacturing Engineers, Dearborn, MI, (1994)

43. C. H. Lee and H. Huh, Blank Design and Strain Prediction of Automobile Stamping Parts by an Inverse Finite Element Approach, Journal of Materials Processing Technology, Vol. 63, pp.645-650, (1997)

44. Tae Wan Ku, Hak Jin Lim, Han Ho Choi, Sang Moon Hwang and Beom Soo Kang, Implementation of Backward Tracing Scheme of the FEM to Blank Design in Sheet Metal Forming, Journal of Materials Processing Technology, Vol. 111, pp.90-97, (2001)

45. H. B. Shim and K. C. Son, Optimal Blank Design for the Drawing of Arbitrary Shapes by the Sensitivity Method, Journal of Engineering Materials and Technology, Vol. 123, October, pp.468-475, (2001)

46. H. Shim and K. Son, Optimal blank shape design by the iterative sensitivity method, Proc Instn Mech Engrs Vol 216 Part B: J Engineering Manufacture, 867-878, (2002)

47. Hae Chang Gea and Rajesh Ramamurthy, Blank design optimization on deep drawing of square shells, IIE Transactions (1998) 30, 913-921

48. Adrian Scott-Murphy, S. Kalyanasundaram, M. Cardew-Hall, A hybrid draw die optimization technique for sheet metal forming, Journal of Manufacturing Sceince and Engineering, August (2004), Vol.126, 582-592

49. Yang Jian-hua, Chen Jun, He Dan-nong, Ruan Xue-yu, Numerical simulation of twostage deep drawing of complex-shaped parts, Trans. Nonferrous Met. Soc. China, Vol. 13, No. 4, August (2003), 839-848 
50. K. Chung, F. Barlat, J. C. Brem, D. J. Lege and O. Richmond, Blank shape design for a planar anisotropic sheet based on ideal forming design theory and FEM analysis, Int. J. Mech. Sci. Vol. 39, No. 1, (1997), 105-120

51. Frank W. Wilson, High-velocity Forming of Metals, American Society of Tool and Manufacturing Engineers, Prentice-hall, Inc., Englewood Cliffs, N. J., (1964)

52. A. A. Ezra, Principles and Practice of Explosive Metalworking, Industrial Newspapers Limited, London, England, (1973)

53. Glenn S. Daehn, Vincent J. Vohnout and Larry DuBois, Improved Formability with Electromagnetic Forming: Fundamentals and a Practical Example, Sheet Metal Forming Technology, Edited by Mahmoud Y. Demeri, The Minerals, Metals \& Materials Society, pp.105-116, (1999)

54. Manish Kamal, A uniform pressure electromagnetic actuator for forming flat sheets, $\mathrm{PhD}$ dissertation, The Ohio State University, Columbus, OH, 2005

55. Mala Seth Dehra, High velocity formability and factors affecting it, PhD dissertation, The Ohio State University, Columbus, OH, 2006

56. Peihui Zhang, Joining enabled by high velocity deformation, $\mathrm{PhD}$ dissertation, The Ohio State University, Columbus, OH, 2003

57. Richard T. Weidner, Physics, Allyn and Bacon, Inc., Needham Heights, MA(1989)

58. F. W. Grover, Inductance Calculations: Working Formulas and Tables, D. Van Nostrand Co., New York, NY, (1946)

59. Francis C. Moon, Magneto-solid Mechanics, John Wiley \& Sons, Inc., (1984)

60. L. V. Belyy, S. M. Fertik and L. T. Khimenko, Electromagnetic Metal Forming Handbook, (A Translation of the Russian Book: Sprvochnik Po Magnitno-impul' Snoy Obrabotke Metallov), Translated by M. M. Altynova and Glenn S. Daehn, Columbus, $\mathrm{OH},(1996)$

61. Yuri V. Batygin and Glenn S. Daehn, The Pulse Magnetic Fields for Progressive Technologies, Kharkov, Columbus, (1999)

62. Anter El-Azab, Mark Garnich, Ashish Kapoor, Modeling of the electromagnetic forming of sheet metals: state-of-the-art and future needs, Journal of Materials Processing Technology, 142 (2003) 744-754

63. Dino A. Oliveira and M. J. Worswick, Simulation of electromagnetic forming of aluminum alloy sheet, SAE technical paper series, 2001-01-0824 
64. Hemant M. Panshikar, Computer modeling of electromagnetic forming and impact welding, MS thesis, The Ohio State University, Columbus, OH, 2000

65. A.G. Mamalis, D.E. Manolakos, A.G. Kladas, A.K. Koumoutsos, Electro-magnetic tooling for metal forming and powder compaction: numerical simulation, Proceedings of the $1^{\text {st }}$ International Conference on High Speed Forming, March 31April 1, 2004, Dortmund, Germany, 143-154

66. J. Unger, M. Stiemer, B. Svendsen, H. Blum, Multifield modeling of electromagnetic metal forming processes, Journal of Materials Processing Technology, 177 (2006), 270-273

67. D. A. Oliveira, M.J. Worswick, M. Finn, D. Newman, Electromagnetic forming of aluminum alloys sheet: free-form and cavity fill experiments and model, Journal of Materials processing Technology, 170 (2005), 350-362

68. Ph. Conraux, M. Pignol, V. Robin, J. M. Bergheau, 3D finite element modeling of electromagnetic forming processes, Proceedings of the $2^{\text {nd }}$ International Conference on High Speed Forming, March 20-21, 2006, Dortmund, Germany, 73-82

69. William D. Callister, Materials Science and Engineering: An Introduction, John Wiley \& Sons, Inc., (1997)

70. Amit A. Tamhane, Marina M. Altynova and Glenn S. Daehn, Effect of Sample Size on Ductility in Electromagnetic Ring Expansion, Scripta Materialia, Vol. 34, 8, pp. 1345-1350, (1996)

71. V. S. Balanethiram and G. S. Daehn, Hyperplasticity-Increased Forming Limits at High Workpiece Velocities, Scripta Metallurgica, Vol.31, pp. 515-520, (1994)

72. X. Hu and G. S. Daehn, Effect of Velocity on Localization in Uniaxial Tension, Acta Materialia, Vol. 44, pp.1021-1033, (1996)

73. C. Fressengeas and A. Molinari, Inertia and Thermal Effects on the Localization of Plastic Flow, Acta Metallurgica Materialia, Vol.33, 3, pp.387-396, (1985)

74. I. M. Fyfe and A. M. Rajendran, Dynamic Pre-strain and Inertia effects on the Fracture of Metals, Journal of the Mechanics and Physics of Solids, Vol. 28, pp.1726, (1980)

75. G. Regazzoni, J. N. Johnson and P. S. Follanbsbee, Theoretical study of the dynamic tensile test, Journal of Applied Mechanic, Vol. 53, September, pp. 519-528, (1986)

76. A Needleman and V. Tevergaard, Analyses of Plastic Flow Localization in Metals, Applied Mechanics Reviews, Vol.45, pp. S3-S15, (1992) 
77. P. S. Follansbee and U. F. Kocks, A Constitutive Description of the Deformation of Cooper Based on the Use of the Mechanical Threshold Stress as an Internal State Variable, Acta Metallurgica Materialia, Vol.36, 1, pp. 81-93, (1988)

78. E. J. Bruno, Editor, High-velocity forming of metals, American Society of Tool and Manufacturing Engineers, Dearborn, MI, (1968)

79. Vincent Vohnout, Glenn Daehn, Reducing stamped part development time with pulsed energy assistance, www.osu.edu/hyperplasticity, 2004

80. Xianjin Wang, Jian yao, Xuanshan Lu, Formability of metal sheet (in Chinese), University of Science and Technology Beijing, (1987)

81. Y. Hishida and R. H. Wagoner, Experimental Analysis of Blank Holding Force Control in Sheet Forming, SAE Paper 930285, Sheet Metal Stamping Symposium, SAE SP-994, pp. 93-100, Warrendale, PA, (1993)

82. Fuh-Kuo Chen and Bai-hong Chiang, Three-Dimensional Finite Element Analysis for the Stamping of a Motorcycle Oil Tank, Journal of Manufacturing Science and Engineering, Vol. 120, November, pp. 770-730, (1998)

83. Yang Hu, Changqing Du and Li Zhang, Numisheet'93 2D Draw Bending Springbuck Simulation Revisit Using LS-Dyna and LS-Nike, Sheet Metal Forming Technology, Edited by Mahmoud Y. Demeri, The Minerals, Metals \& Materials Society, pp.179186, (1999)

84. A. Scott-Murphy, M. Cardew-Hall, P. Hodgson and S. Kalyanasundaram, FEM Optimization of Sheet Metal Forming, Sheet Metal Forming Technology, Edited by Mahmoud Y. Demeri, The Minerals, Metals \& Materials Society, pp.77-92, (1999)

85. T. Ohata, Y. Nakamura, T. Katayama, E. Nakamachi and K. Nakano, Development of Optimum Process Design System by Numerical Simulation, Journal of Materials Processing Technology, Vol. 60, pp.543-548, (1996)

86. www.maxwell.com, 2006

87. www.matweb.com. 2005

88. J. L. Duncan, Jack Hu, Z. Marciniak, Mechanics of sheet metal forming, Second edition, Butterworth-Heinemann, (2002)

89. R. Kaibyshev, O. Sitdikov, I. Mazurina, D.R. Lesuer, Deformation behavior of a 2219 Al alloy, Materials Science and Engineering A334 (2202), 104-113

90. M. Jain, J. Allin, M. J. Bull, Deep drawing characteristics of automotive aluminum alloys, Materials Science and Engineering A256 (1998) 69-82 
91. Prakash Sonis, N. Venkata Reddy, G. K. Lal, On multistage deep drawing of axisymmetric components, Journal of Manufacturing Science and Engineering, May 2003, Vol. 125, 352-362

92. Daw-Kwei Leu, Jen-Yu Wu, A simplified Approach to Estimate Limiting Drawing ratio and Maximum Drawing Load in Cup Drawing, Journal of Engineering Materials and Technology, January 2004, Vol. 126, 116-122

93. Brad L. Kinsey, Jian Cao, An analytical model for tailor welded blank forming, Journal of Manufacturing Science and Engineering, May 2003, Vol. 125, 344-351

94. A. S. Korhonen, Drawing force in deep drawing of cylindrical cup with flat-nosed punch, Journal of Engineering for Industry, February 1982, Vol. 104, 29-37

95. S. Fukui, K. Yoshida, S. Kobayashi, Sheet metal forming research in Japan, Journal of Engineering for Industry, February 1966, 101-110

96. D. C. Chiang, Shiro Kobayashi, the effect of anisotropy and work-hardening characteristics on the stress and strain distribution in deep drawing, Journal of Engineering for Industry, February 1966, 443-448

97. Mark Colgan, John Monaghan, Deep drawing process: analysis and experiment, Journal of Materials Processing Technology, 132 (2003) 35-41

98. Min Wan, Yu-Ying Yang, Shuo-Ben Li, Determination of fracture criteria during the deep drawing of conical cups, Journal of Materials Processing Technology, 114 (2001), 109-113

99. Min Wan, Yu-Ying Yang, Shuo-Ben Li, Determination of the limiting drawing coefficient in the deep drawing of conical cups, Journal of Materials Processing Technology, 114 (2001), 114-117

100. A. Hofmann, Deep drawing of process optimized blanks, Journal of Materials Processing Technology, 119 (2001), 127-132

101. J. Zhao, H. Q. Cao, L. X. Ma, F. Q. Wang, S. B. Li, Study on intelligent control technology for the deep drawing of an axi-symmetric shell part, Journal of Materials Processing Technology, 151 (2004), 98-104

102. C. Loganathan, R. Narayanasamy, Effect of mechanical properties on the wrinkling behavior of three different commercially pure aluminum grades when drawn through conical and tractrix dies, Materials Science and Engineering A 406, (2005), 229-253 
103. Lei Junxiang, Kang Yonglin, Prediction and control of both wrinkle and fracture limits on cylindrical cup multi-deep-drawing, Chinese Journal of Mechanical Engineering, Vol. 12, No. 4, (1999), 288-296

104. Jian Cao, Xi Wang, Frances Joe Mills, Characterization of sheet buckling subjected to controlled boundary constraints, Journal of Manufacturing Science and Engineering, August 2002, Vol. 124, 493-501

105. R. Narayanasamy, R. Sowerby, Wrinkling behavior of cold-rolled sheet metals when drawing through a tractrix die, Journal of Materials Processing Technology, 49 (1995), 199-211

106. Youngsuk Kim, Youngjin Son, Study on wrinkling limit diagram of anisotropic sheet metals, Journal of Materials Processing Technology, 97 (2000), 88-94

107. Xi Wang, Jian Cao, An analytical prediction of flange wrinkling in sheet metal forming, Journal of Manufacturing Processes, Vol.2, No. 2, (2000), 100-107

108. William H. Gourdin, Analysis and assessment of electromagnetic ring expansion as a high-strain-rate test, J. Appl. Phys. 65 (2), January 1989, 411-422

109. J. Jablonski, R. Winkler, Analysis of the electromagnetic forming process, Int. J. Mech. Sci. Vol. 20, 315-325

110. Glenn Daehn, An Approach to Flexible Metal Forming System Design Based on Line Impulse Actuators, www.osu.edu/hyperplasticity, 2005

111. Daoming Li, Zhen Guo, Alexandru Riposan and Amit Ghosh, Advances in warm forming technology for aluminum alloys sheets, http://www.mse.engin.umich.edu/research/groups/ghosh/publications/2004

112. Hong Yao, Brad L. Kinsey, Jian Cao, Rapid design of corner restrain force in deep drawn rectangular parts, Internationals Journal of Machine Tools \& Manufacture, 40, (2000), 113-131

113. Hong Yao, Jian Cao, Assessment of corner failure depths in the deep drawing of $3 D$ panels using simplified $2 d$ numerical and analytical models, Journal of Manufacturing Science and Engineering, May 2001, Vol. 123, 248-257

114. M. J. Saran, Y. T. Keum and R. H. Wagoner, Section analysis with irregular tools and arbitrary draw-in conditions for numerical simulation of sheet forming, Int. J. Mech. Sci., Vol. 33, No. 11, (1991), 893-909

115. Sen Jiang, Springback investigations, MS thesis, The Ohio State University, Columbus, OH, 1997 
116. Annika Nilsson, Lars Melin, Claes Magnusson, Finite-element simulation of V-die bending: a comparison with experimental results, Journal of Materials Processing Technology, 65 (1997), 52-58

117. A. Forcellese, L. Fratini, F. Gabrielli, F. Micari, the evaluation of springback in 3D stamping and coining processes, Journal of Materials Processing Technology, 8081 (1998), 108-112

118. Luca Tomesani, Quality control in plane-strain bead-forming processes through the load-displacement curve, Journal of Materials Processing Technology, 7 (1998), $145-152$

119. You-Min Huang, Tsung-Chia Chen, An elasto-plastic finite-element analysis of sheet metal camber process, Journal of Materials Processing Technology, 140 (2003), $432-440$

120. Mala Seth, High velocity formability of high strength steel sheet, MS thesis, The Ohio State University, Columbus, OH, 2003 